\title{
A Synthetic Entry to Polyfunctionalized Molecules through the [3+2]-Cycloaddition of Thiocarbonyl Ylides
}

Franz-Lucas Haut, ${ }^{\dagger}$ Christoph Habiger, ${ }^{\dagger}$ Klaus Speck, ${ }^{\ddagger}$ Klaus Wurst,${ }^{\S}$ Peter Mayer, ${ }^{\ddagger}$ Johannes Nepomuk Korber, ${ }^{\dagger}$ Thomas Müller ${ }^{\dagger}{ }^{\text {and }}$ Thomas Magauer* ${ }^{\dagger}$

$\dagger$ Institute of Organic Chemistry and Center for Molecular Biosciences, Leopold-Franzens-University Innsbruck, Innrain 80-82, 6020 Innsbruck

\Department of Chemistry and Pharmacy, Ludwig-Maximilians-University Munich, Butenandtstrasse 5-13, 81377 Munich, Germany

${ }^{\S}$ Institute of General, Inorganic \& Theoretical Chemistry, Leopold-Franzens-University Innsbruck, Innrain 8082, 6020 Innsbruck, Austria

- Supporting Information - 


\section{Table of Contents}

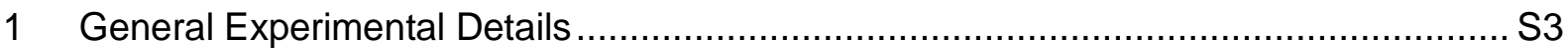

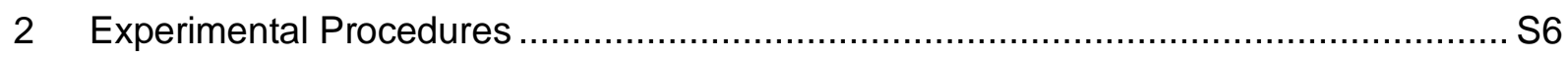

2.1 Precursor and Dipole Synthesis ............................................................. 6

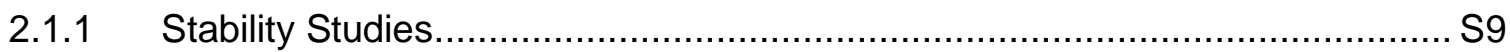

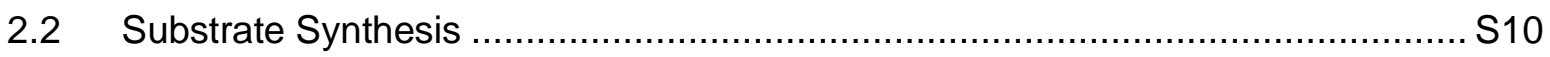

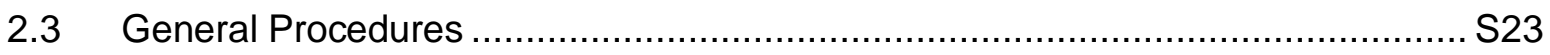

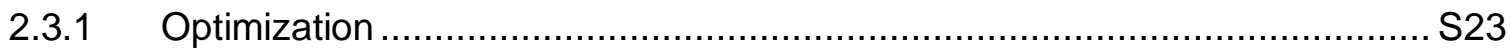

2.3.2 General Procedure 1: High-Pressure (GP1) ….................................... $S 23$

2.3.3 General Procedure 2: Heat (GP2) .................................................... S24

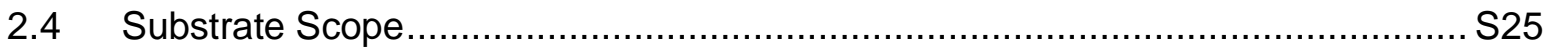

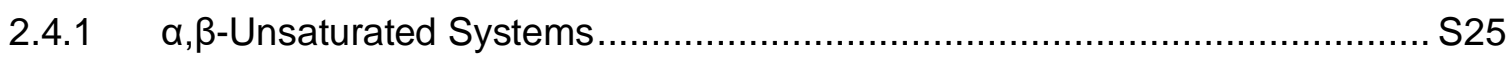

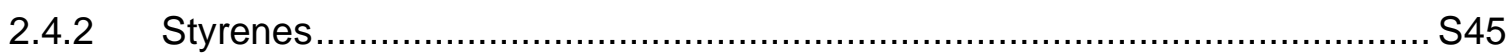

2.4.3 1,4- vs. 1,6-Addition and [6+3]-Cycloaddition ......................................... S51

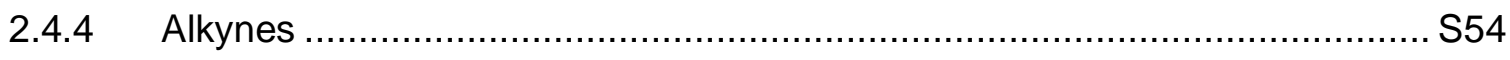

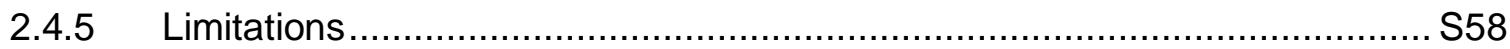

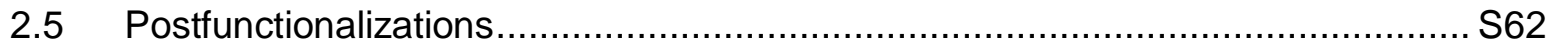

2.5.1 Quaternary Carbon Centers ........................................................... 570

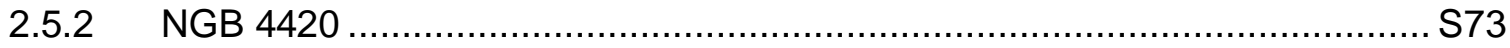

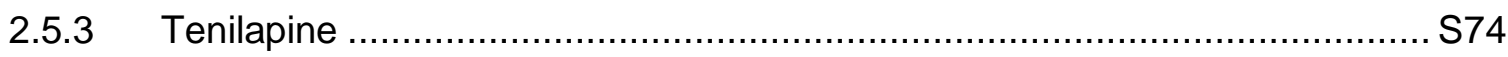

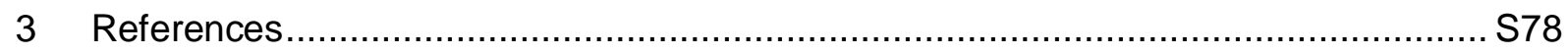

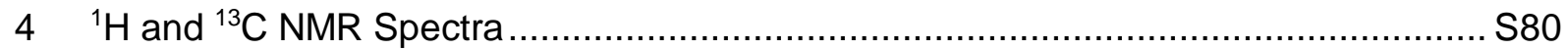

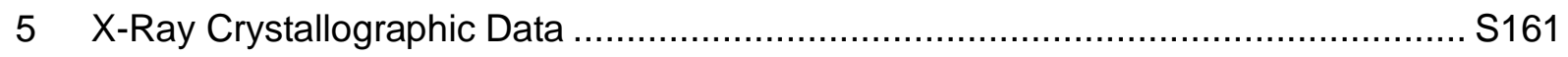




\section{General Experimental Details}

All reactions were performed in flame-dried glassware fitted with rubber septa under a positive pressure of argon, unless otherwise noted. Air- and moisture-sensitive liquids were transferred via syringe or stainless-steel cannula through rubber septa. Solids were added under inert gas counter flow or were dissolved in appropriate solvents. Low temperaturereactions were carried out in a Dewar vessel filled with a cooling agent: acetone/dry ice $\left(-78^{\circ} \mathrm{C}\right)$, sodium chloride/ice $\left(-18^{\circ} \mathrm{C}\right)$ or distilled water/ice $\left(0^{\circ} \mathrm{C}\right)$. Reaction temperatures above room temperature were conducted in a heated oil bath or a metal block. The reactions were magnetically stirred and monitored by NMR spectroscopy or analytical thin-layer chromatography (TLC), using aluminum plates precoated with silica gel $(0.25 \mathrm{~mm}, 60$ - $\AA$ pore size, Merck) impregnated with a fluorescent indicator $(254 \mathrm{~nm})$. TLC plates were visualized by exposure to ultraviolet light (UV), were stained by submersion in aqueous potassium permanganate solution $\left(\mathrm{KMnO}_{4}\right)$ and ceric ammonium molybdate solution (CAM) and were developed by heating with a heat gun. Flash-column chromatography was performed as described by Still et al., ${ }^{1}$ employing silica gel (60 $\AA, 40-63 \mu \mathrm{m}$, Merck). The yields refer to chromatographically and spectroscopically $\left({ }^{1} \mathrm{H}\right.$ and ${ }^{13} \mathrm{C}$ NMR $)$ pure material.

\section{Materials}

Tetrahydrofuran (THF) and diethyl ether $\left(\mathrm{Et}_{2} \mathrm{O}\right)$ were distilled under $\mathrm{N}_{2}$ atmosphere from sodium and benzophenone prior to use. Dichloromethane $\left(\mathrm{CH}_{2} \mathrm{Cl}_{2}\right)$, triethylamine $\left(\mathrm{Et}{ }_{3} \mathrm{~N}\right)$, diisopropylamine (DIPA) and Hünig's base (DIPEA) were distilled under nitrogen atmosphere from $\mathrm{CaH}_{2}$ prior to use. Dimethyl sulfoxide (DMSO), acetonitrile (MeCN), acetone, toluene, chloroform $\left(\mathrm{CHCl}_{3}\right)$ and methanol $(\mathrm{MeOH})$ were purchased from Acros Organics as 'extra dry' reagents and used as received. All other reagents and solvents were purchased from chemical suppliers (Sigma-Aldrich, TCI, Acros Organics or others) and were used as received. Solvents for extraction, crystallization and flash column chromatography were purchased in technical grade and distilled under reduced pressure prior to use. The molarity of $n$-butyllithium and $t$-butyllithium solutions was determined by titration against diphenylacetic acid as an indicator (average of three determinations). ${ }^{2}$

\section{NMR spectroscopy}

NMR spectra were measured on a Bruker Avance Neo $400 \mathrm{MHz}$ spectrometer and a Bruker Avance II $600 \mathrm{MHz}$ spectrometer at Leopold-Franzens-University Innsbruck (LFU Innsbruck) or a Bruker Avance III HD $400 \mathrm{MHz}$ spectrometer equipped with a CryoProbeTM, a Varian VXR $400 \mathrm{MHz}$ spectrometer, a Bruker AMX $600 \mathrm{MHz}$ spectrometer or a Bruker Avance HD $800 \mathrm{MHz}$ spectrometer at Ludwig-Maximilians-University Munich (LMU Munich). Proton chemical shifts are expressed in parts per million (ppm, $\delta$ scale) and are referenced to 
residual proton in the NMR solvent $\left(\mathrm{CHDCl}_{2}: \delta 5.32 ; \mathrm{CHCl}_{3}: \delta 7.26\right)$. Carbon chemical shifts are expressed in parts per million ( $\delta$ scale, assigned carbon atom) and are referenced to the carbon resonance of the NMR solvent $\left(\mathrm{CD}_{2} \mathrm{Cl}_{2}: \delta 53.84 ; \mathrm{CDCl}_{3}: \delta 77.16\right) .{ }^{1} \mathrm{H} \mathrm{NMR}$ spectroscopic data are reported as follows: Chemical shift in ppm (multiplicity, coupling constants $J(\mathrm{~Hz})$, integration intensity, assigned proton). The multiplicities are abbreviated with $\mathrm{s}$ (singlet), $\mathrm{d}$ (doublet), $\mathrm{t}$ (triplet), $\mathrm{q}$ (quartet) and $\mathrm{m}$ (multiplet). In case of combined multiplicities, the multiplicity with the larger coupling constant is stated first. Except for multiplets, the chemical shift of all signals, as well for centrosymmetric multiplets, is reported as the center of the resonance range. Additionally, to ${ }^{1} \mathrm{H}$ and ${ }^{13} \mathrm{C}-\mathrm{NMR}$ measurements, $2 \mathrm{D}$ NMR techniques such as homonuclear correlation spectroscopy (COSY), total correlation spectroscopy (TOCSY), heteronuclear single quantum coherence (HSQC) and heteronuclear multiple bond coherence ( $\mathrm{HMBC}$ ) were used to assist signal assignment. For further elucidation of 3D structures of the products, nuclear Overhauser enhancement spectroscopy (NOESY) was conducted. Coupling constants $\mathrm{J}$ are reported in $\mathrm{Hz}$. All raw fid files were processed, and the spectra analyzed using the software MestReNova 10.0.2 from Mestrelab Research S. L.

\section{IR spectroscopy}

IR spectra were recorded on a PerkinElmer Spectrum BX II FT-IR system. If required, substances were dissolved in $\mathrm{CH}_{2} \mathrm{Cl}_{2}$ or $\mathrm{CDCl}_{3}$ prior to direct application on the ATR unit. Data are represented as follows: frequency of absorption $\left(\mathrm{cm}^{-1}\right)$, and intensity of absorption ( $v s=$ very strong, $s=$ strong,$m=$ medium, $w=$ weak, $b r=$ broad).

\section{Mass spectrometry}

High-resolution mass spectra (HRMS) were recorded on a Varian MAT CH7A or a Varian MAT $711 \mathrm{MS}$ instrument by electron impact (EI) or electrospray ionization (ESI) techniques at the LMU Munich or a Thermo Scientific ${ }^{\mathrm{TM}}$ LTQ Orbitrap XL ${ }^{\mathrm{TM}}$ Hybrid Ion Trap-Orbitrap Mass Spectrometer at the LFU Innsbruck. The ionization and detection of tetrahydrothiophenes by ESI technique could be significantly improved by the addition of silver(I) nitrate to give the corresponding silver adducts (only the ${ }^{107} \mathrm{Ag}$-adduct is reported). 


\section{Optical rotation}

Optical rotation values were recorded on a PerkinElmer 241 or Anton Paar MCP 200 polarimeter. The specific rotation is calculated as follows:

$$
[\alpha]_{\lambda}^{\varphi}=\frac{[\alpha] \cdot 100}{c \cdot d}
$$

Thereby, the wave length $\lambda$ is reported in $\mathrm{nm}$ and the measuring temperature $\phi$ in ${ }^{\circ} \mathrm{C} . \alpha$ represents the recorded optical rotation at the apparatus, $c$ the concentration of the analyte in $10 \mathrm{mg} / \mathrm{mL}$ and $d$ the length of the cuvette in $\mathrm{dm}$. Thus, the specific rotation is given in $10^{-1} \cdot \mathrm{deg} \cdot \mathrm{cm}^{2} \cdot \mathrm{g}^{-1}$. Usage of the sodium $D$ line $(\lambda=589 \mathrm{~nm})$ is indicated by $D$ instead of the wavelength in $\mathrm{nm}$. The respective concentration as well as the solvent is reported at the relevant section of the experimental section.

\section{Melting Point}

Melting points (MP) were measured with an SRS-MPA120 EZ-Melt Melting Point Apparatus in open glass capillaries and are uncorrected.

\section{High-Pressure Set-Up}

High-pressure reactions were performed in custom-made PTFE reaction vials in an emulsion high-pressure machine from Andreas Hofer Hochdrucktechnik $\mathrm{GmbH}$ (up to $14 \mathrm{kbar}$, piston $\varnothing 25 \mathrm{~mm}$, stroke $95 \mathrm{~mm}$ ) equipped with a Julabo MA-4 circulation thermostat (up to $50{ }^{\circ} \mathrm{C}$ ). 


\section{Experimental Procedures}

\subsection{Precursor and Dipole Synthesis}

Thioether S-2

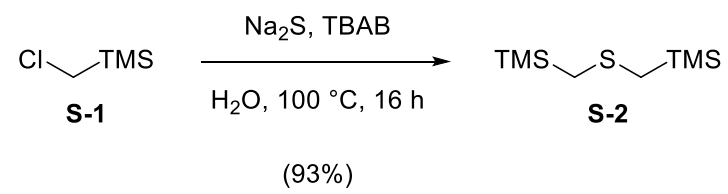

To a suspension of sodium sulfide hydrate $\left(9.0 \mathrm{~g}, 76 \mathrm{mmol}, 1\right.$ equiv, $\left.65 \% \mathrm{Na}_{2} \mathrm{~S}\right)$ in water $(27 \mathrm{~mL})$ were subsequently added tetrabutylammonium bromide $(0.49 \mathrm{~g}, 1.5 \mathrm{mmol}$, 0.02 equiv) and (chloromethyl)trimethylsilane (S-1, $21 \mathrm{~mL}, 0.15 \mathrm{~mol}, 2.0$ equiv) at $23^{\circ} \mathrm{C}$. Upon addition, the reaction mixture was heated to $100^{\circ} \mathrm{C}$ in a sealed pressure flask. After 16 hours, water $(50 \mathrm{~mL})$ and pentane $(50 \mathrm{~mL})$ were added and the layers were separated. The aqueous layer was extracted with pentane $(2 \times 50 \mathrm{~mL})$, the combined organic layers were dried over sodium sulfate, filtered and concentrated $\left(40^{\circ} \mathrm{C}, 50 \mathrm{mbar}\right)$ to give pure thioether S-2 (15 g, $71 \mathrm{mmol}, 93 \%)$ as a colorless oil. The obtained analytical data was in full agreement with those reported in literature. ${ }^{3}$

TLC (pentane): $R_{\mathrm{f}}=0.38\left(\mathrm{KMnO}_{4}\right)$.

${ }^{1} \mathrm{H}$ NMR $\left(\mathrm{CDCl}_{3}, 400 \mathrm{MHz}\right): \delta=1.83(\mathrm{~s}, 4 \mathrm{H}), 0.08(\mathrm{~s}, 18 \mathrm{H})$.

${ }^{13} \mathrm{C}$ NMR $\left(\mathrm{CDCl}_{3}, 101 \mathrm{MHz}\right): \delta=24.1,-1.5$.

HRMS (ESI) calc. for $\mathrm{C}_{8} \mathrm{H}_{22}{ }^{107} \mathrm{AgSSi} 2\left[\mathrm{M}+{ }^{107} \mathrm{Ag}\right]^{+:}: 313.0026$; found: 313.0014 .

\section{Sulfoxide 1a}

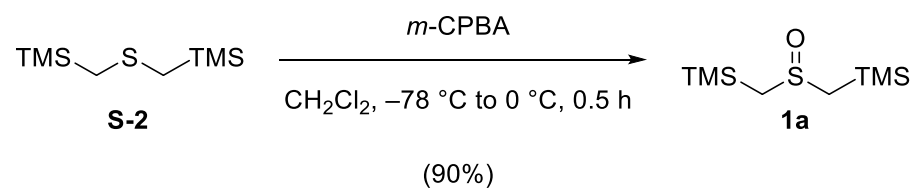

To a solution of thioether S-2 (3.76 g, $18.2 \mathrm{mmol}, 1$ equiv) in dichloromethane $(40 \mathrm{~mL})$ was added a solution of meta-chloroperbenzoic acid $(4.60 \mathrm{~g}, 20.0 \mathrm{mmol}, 1.10$ equiv) in dichloromethane $(40 \mathrm{~mL})$ through a dropping funnel over a period of 30 minutes at $-78{ }^{\circ} \mathrm{C}$. The resulting milky suspension was allowed to warm to $0{ }^{\circ} \mathrm{C}$. After ten minutes, ice-cold water $(100 \mathrm{~mL})$ and dichloromethane $(70 \mathrm{~mL})$ were added. The layers were separated, the organic layer was washed with saturated aqueous sodium bicarbonate-solution $(3 \times 100 \mathrm{~mL})$, 
dried over sodium sulfate, filtered and concentrated at $0{ }^{\circ} \mathrm{C}$ under reduced pressure to give sulfoxide $1 \mathrm{a}$ as colorless oil $(3.65 \mathrm{~g}, 90 \%)$, which was used without further purification.

TLC (1\% methanol in dichloromethane): $R_{\mathrm{f}}=0.54\left(\mathrm{KMnO}_{4}\right)$.

${ }^{1} \mathrm{H}$ NMR $\left(\mathrm{CDCl}_{3}, 400 \mathrm{MHz}\right) \delta=2.41(\mathrm{~d}, J=13.5 \mathrm{~Hz}, 2 \mathrm{H}), 2.14(\mathrm{~d}, J=13.5 \mathrm{~Hz}, 2 \mathrm{H}), 0.20(\mathrm{~s}$, $18 \mathrm{H})$.

${ }^{13} \mathrm{C} \mathrm{NMR}\left(\mathrm{CDCl}_{3}, 101 \mathrm{MHz}\right) \delta=47.8,-0.7$

IR (Diamond-ATR, $\left.\mathrm{CDCl}_{3}\right): \tilde{v}_{\max }=2955(w), 2897(w), 1414(w), 1249(m), 1088(w)$, $1025(m), 846(v s), 770(w), 732(w), 698(s)$.

Note: Sulfoxide 1a could be carefully handled at $0^{\circ} \mathrm{C}$, however it tended to decompose at $23^{\circ} \mathrm{C}$ due to sila-Pummerer rearrangement (see 2.1.1) or polymerization pathways of spontaneously formed dipole. Storage of 1a was possible at $-30^{\circ} \mathrm{C}$ for over four weeks (colorless, crystalline solid which melts upon warming up to $23^{\circ} \mathrm{C}$ ).

\section{Thioether S-4}

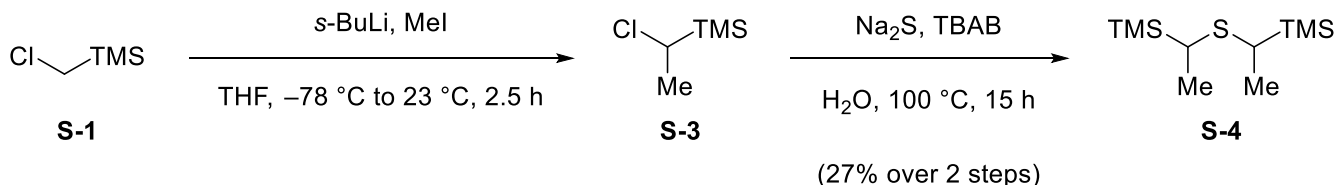

Thioether S-4 was synthesized based on a slightly modified procedure described by Winter and Trauner. ${ }^{4}$ To a solution of (chloromethyl)trimethylsilane (S-1, $10 \mathrm{~mL}, 72 \mathrm{mmol}, 1$ equiv) in tetrahydrofuran $(250 \mathrm{~mL})$ was dropwise added a solution of sec-butyllithium $(51 \mathrm{~mL}$, $72 \mathrm{mmol}$, 1.0 equiv, $1.4 \mathrm{M}$ in cyclohexane) at $-78^{\circ} \mathrm{C}$. Upon addition, the yellow reaction mixture was stirred for one hour at $-78^{\circ} \mathrm{C}$. Then iodomethane $(4.5 \mathrm{~mL}, 72 \mathrm{mmol}, 1.0$ equiv) was added dropwise and the reaction mixture was allowed to warm to $23^{\circ} \mathrm{C}$. After 1.5 hours, the reaction mixture was diluted with diethyl ether $(50 \mathrm{~mL})$ and the organic layer was washed with water $(6 \times 150 \mathrm{~mL})$. The organic phase was dried over magnesium sulfate, filtered and carefully concentrated $\left(40^{\circ} \mathrm{C}, 300\right.$ mbar). The remaining solvent was removed by distillation via Vigreux column (atmospheric pressure, $160^{\circ} \mathrm{C}$ ) to yield chloride S-3 $(5.6 \mathrm{~g}, 92 \mathrm{wt} \%$ in cyclohexane) as a pale-yellow liquid. The obtained product was used in the next step without further purification.

Chloride S-3 (5.6 g, $38 \mathrm{mmol}, 2.0$ equiv) was placed in a pressure tube followed by the addition of sodium sulfide $\left(2.4 \mathrm{~g}, 18 \mathrm{mmol}, 1\right.$ equiv, $\left.65 \% \mathrm{Na}_{2} \mathrm{~S}\right)$, tetrabutylammonium bromide ( $0.73 \mathrm{~g}, 2.3 \mathrm{mmol}, 0.12$ equiv) and water $(8 \mathrm{~mL})$. Upon addition, the vigorously 
stirred reaction mixture was heated to $100^{\circ} \mathrm{C}$. After 15 hours, the reaction mixture was allowed to cool to $23^{\circ} \mathrm{C}$ and was diluted with cyclohexane $(10 \mathrm{~mL})$ and water $(10 \mathrm{~mL})$. The layers were separated and the aqueous phase was extracted with cyclohexane $(50 \mathrm{~mL})$. The combined organic extracts were washed with saturated aqueous sodium bicarbonate solution $(50 \mathrm{~mL})$, dried over magnesium sulfate, filtered and concentrated $\left(40^{\circ} \mathrm{C}, 50 \mathrm{mbar}\right)$. The crude product was purified by flash-column chromatography on silica gel (pentane) to yield thioether S-4 (2.3 g, 27\% over two steps) as a colorless liquid. The obtained analytical data were in full agreement with those reported in literature. ${ }^{4}$

TLC (pentane): $R_{\mathrm{f}}=0.55\left(\mathrm{KMnO}_{4}\right)$.

\section{Sulfoxide 1b}

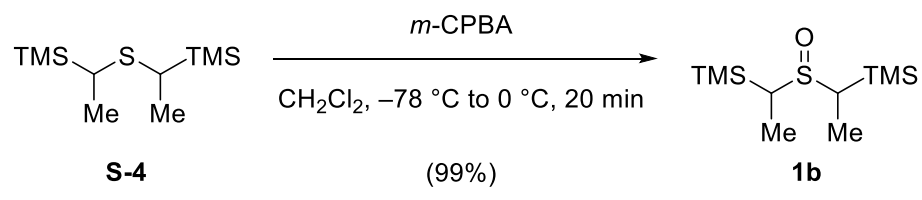

To a solution of thioether S-4 (422 mg, $1.80 \mathrm{mmol}$, 1 equiv) in dichloromethane $(4.5 \mathrm{~mL})$ was added a solution of meta-chloroperbenzoic acid (460 mg, $1.98 \mathrm{mmol}, 1.10$ equiv) in dichloromethane $(4.5 \mathrm{~mL})$ dropwise over a period of ten minutes at $-78^{\circ} \mathrm{C}$. The resulting milky suspension was allowed to warm to $0^{\circ} \mathrm{C}$. After 20 minutes, ice-cold water $(15 \mathrm{~mL})$ was added and the layers were separated. The organic layer was washed with saturated aqueous sodium bicarbonate-solution $(3 \times 15 \mathrm{~mL})$, dried over sodium sulfate, filtered and concentrated at $0{ }^{\circ} \mathrm{C}$ under reduced pressure to give sulfoxide $1 \mathrm{~b}$ as colorless oil (450 mg, 99\%), which was used without further purification.

TLC (1\% methanol in dichloromethane): $R_{\mathrm{f}}=0.23\left(\mathrm{KMnO}_{4}\right)$. 


\subsubsection{Stability Studies}

Sila-Pummerer rearrangement of thioether $1 \mathrm{a}$ to thioacetal S-5

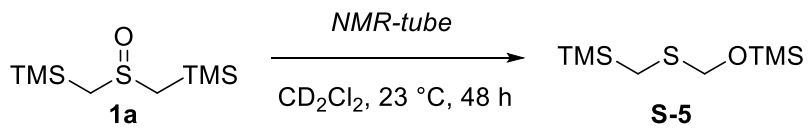

[sila-Pummerer rearrangement]

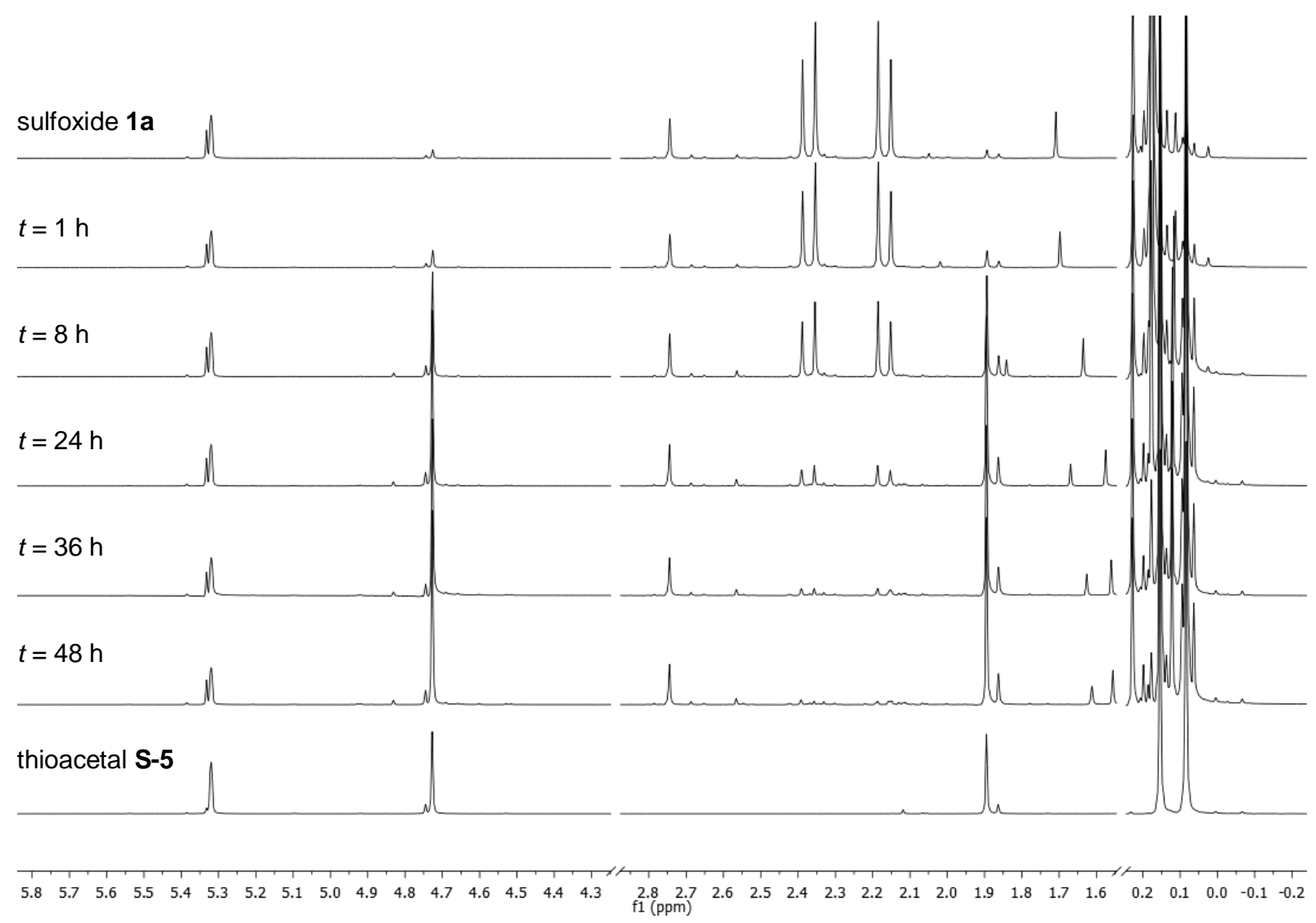

TLC (40\% dichloromethane in pentane): $\mathrm{R}_{\mathrm{f}}=0.44\left(\mathrm{KMnO}_{4}\right)$.

${ }^{1} \mathrm{H}$ NMR $\left(\mathrm{CD}_{2} \mathrm{Cl}_{2}, 400 \mathrm{MHz}\right) \delta=4.73(\mathrm{~s}, 2 \mathrm{H}), 1.89(\mathrm{~s}, 2 \mathrm{H}), 0.15(\mathrm{~s}, 9 \mathrm{H}), 0.08(\mathrm{~s}, 9 \mathrm{H})$.

${ }^{13} \mathrm{C}$ NMR $\left(\mathrm{CD}_{2} \mathrm{Cl}_{2}, 101 \mathrm{MHz}\right) \delta=69.6,16.8,0.1,-1.5$

IR (Diamond-ATR, $\left.\mathrm{CD}_{2} \mathrm{Cl}_{2}\right): \tilde{v}_{\max }=2956(w), 1392(w), 1315(w), 1248(m), 1129(w)$, $1063(s), 835(v s), 751(m), 716(w), 693(m)$.

HRMS (ESI) calc. for $\mathrm{C}_{8} \mathrm{H}_{22}{ }^{107} \mathrm{AgOSSi}{ }_{2}\left[\mathrm{M}+{ }^{107} \mathrm{Ag}\right]^{+:}$: 328.9975; found: 328.9969 . 


\subsection{Substrate Synthesis}

\section{Silyl enol ether S-7}

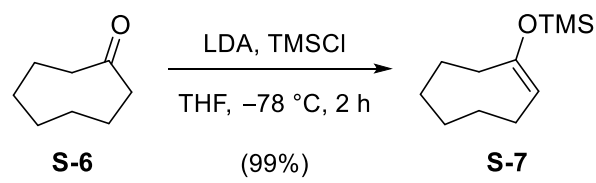

To a solution of diisopropylamine $(1.15 \mathrm{~mL}, 8.08 \mathrm{mmol}, 1.30$ equiv) in tetrahydrofuran $(13 \mathrm{~mL})$ was added $n$-butyllithium $(3.23 \mathrm{~mL}, 8.08 \mathrm{mmol}, 1.30$ equiv, $2.50 \mathrm{M}$ in $n$-hexane) at $-78^{\circ} \mathrm{C}$. After 30 minutes, chlorotrimethylsilane $(1.60 \mathrm{~mL}, 12.4 \mathrm{mmol}, 2.00$ equiv, freshly distilled over calcium hydride) and a solution of cyclooctanone (S-6, $800 \mathrm{mg}, 6.21 \mathrm{mmol}$, 1 equiv) in tetrahydrofuran $(3 \mathrm{~mL})$ were subsequently added at $-78^{\circ} \mathrm{C}$. After one hour, the reaction was allowed to warm to $23^{\circ} \mathrm{C}$ and triethylamine $(2 \mathrm{~mL})$ and a saturated aqueous sodium bicarbonate solution ( $20 \mathrm{~mL}$ ) were subsequently added. The layers were separated, the aqueous layer extracted with cyclohexane $(3 \times 100 \mathrm{~mL})$, the combined organic layers washed with saturated aqueous sodium chloride solution $(50 \mathrm{~mL})$ and dried over sodium sulfate. The dried solution was filtered and concentrated to furnish enol ether S-7 $(1.22 \mathrm{~g}$, $99 \%$ ) as a colorless liquid, which was used in the next step without further purification. ${ }^{5}$

\section{2-Cycloocten-1-one (S-8)}

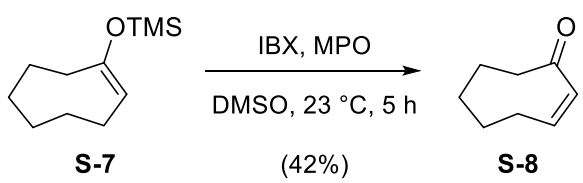

To a solution of enol ether S-7 (1.22 g, $6.15 \mathrm{mmol}, 1$ equiv) in dimethyl sulfoxide $(6 \mathrm{~mL})$ was added a mixture of 2-iodoxybenzoic acid $(2.24 \mathrm{~g}, 8.00 \mathrm{mmol}, 1.30$ equiv) and 4-methoxypyridine $\mathrm{N}$-oxide $(1.00 \mathrm{~g}, 8.00 \mathrm{mmol}, 1.30$ equiv) in dimethyl sulfoxide $(6 \mathrm{~mL})$ at $23^{\circ} \mathrm{C}$. After five hours, saturated aqueous sodium bicarbonate solution ( $\left.30 \mathrm{~mL}\right)$ was added and the layers were separated. The aqueous layer was extracted with diethyl ether $(3 \times 50 \mathrm{~mL})$, the combined organic layers were dried over sodium sulfate, filtered and concentrated. The crude product was purified by flash-column chromatography on silica gel (5\% ethyl acetate in cyclohexane) to furnish 2-cycloocten-1-one (S-8, $315 \mathrm{mg}, 42 \%$ ) as a yellowish liquid. The obtained analytical data were in full agreement with those reported in literature. ${ }^{6}$

TLC $\left(10 \%\right.$ ethyl acetate in cyclohexane): $R_{\mathrm{f}}=0.25\left(\mathrm{KMnO}_{4}\right)$. 


\section{Ethyl carboxylate S-9}

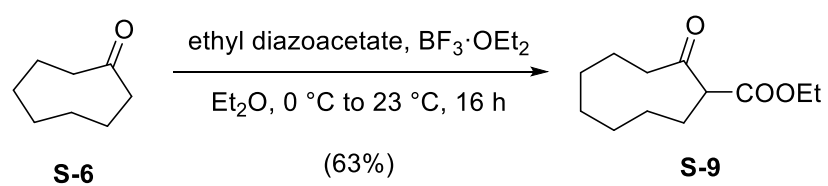

To a solution of cyclooctanone (S-6, $3.00 \mathrm{~g}, 23.3 \mathrm{mmol}, 1$ equiv) in diethyl ether $(50 \mathrm{~mL})$ was added boron trifluoride etherate $(8.98 \mathrm{~mL}, 34.9 \mathrm{mmol}, 1.50$ equiv, $47 \%$ in diethyl ether) at $0{ }^{\circ} \mathrm{C}$. A solution of ethyl diazoacetate $(4.22 \mathrm{~mL}, 34.9 \mathrm{mmol}, 1.50$ equiv, $87 \%$ in dichloromethane) in diethyl ether $(15 \mathrm{~mL})$ was added dropwise over 30 minutes at $0{ }^{\circ} \mathrm{C}$, causing a vigorous evolution of gas. After 30 minutes, the reaction was allowed to warm to $23^{\circ} \mathrm{C}$. After 16 hours, the mixture was cooled down to $0{ }^{\circ} \mathrm{C}$ and saturated aqueous sodium bicarbonate solution was added $(50 \mathrm{~mL})$, the layers separated and the aqueous layer extracted with chloroform $(3 \times 100 \mathrm{~mL})$. The combined organic layers were dried over sodium sulfate, filtered and concentrated. The crude product was purified by flash-column chromatography on silica gel (5\% ethyl acetate in cyclohexane) to furnish ethyl carboxylate S-9 $(3.10 \mathrm{~g}, 63 \%$, 1:1 mixture of keto-enol tautomers) as an orange oil. The obtained analytical data were in full agreement with those reported in literature. ${ }^{7}$

TLC $\left(10 \%\right.$ ethyl acetate in cyclohexane): $R_{f}=0.49\left(\mathrm{KMnO}_{4}\right)$.

\section{Cyclononanone (S-10)}

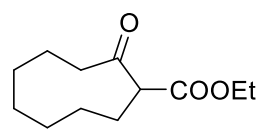

S-9

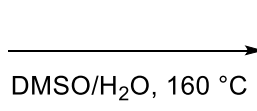

$(65 \%)$

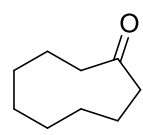

S-10

A solution of carboxylate S-9 (3.10 g, $14.6 \mathrm{mmol}, 1$ equiv) in degassed dimethyl sulfoxide $(15 \mathrm{~mL})$ and water $(3 \mathrm{~mL})$ was heated to $160^{\circ} \mathrm{C}$. After 16 hours, the reaction was allowed to cool down to $23^{\circ} \mathrm{C}$ and water $(50 \mathrm{~mL})$ was added. The aqueous layer was extracted with diethyl ether $(3 \times 100 \mathrm{~mL})$. The combined organic layers were washed with saturated aqueous sodium chloride solution $(50 \mathrm{~mL})$, dried over sodium sulfate, filtered and concentrated. The crude product was purified by flash-column chromatography on silica gel (5\% ethyl acetate in cyclohexane) to yield cyclononanone (S-10, $1.34 \mathrm{~g}, 65 \%$ ) as a yellow liquid. The obtained analytical data were in full agreement with those reported in literature. ${ }^{7}$

TLC $(10 \%$ ethyl acetate in cyclohexane $): R_{\mathrm{f}}=0.62\left(\mathrm{KMnO}_{4}\right)$. 


\section{2-Cyclononen-1-one (S-11)}

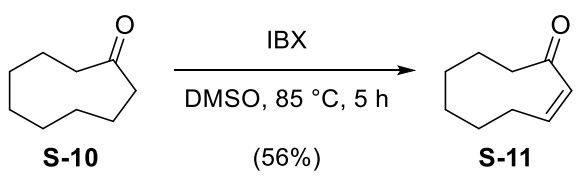

A solution of cyclononanone (S-10, $300 \mathrm{mg}, 2.14 \mathrm{mmol}, 1$ equiv) and 2-iodoxybenzoic acid $\left(1.10 \mathrm{~g}, 3.85 \mathrm{mmol}, 1.80\right.$ equiv) in dimethyl sulfoxide $(13 \mathrm{~mL})$ was heated at $85^{\circ} \mathrm{C}$. After five hours, the reaction was allowed to cool down to $23^{\circ} \mathrm{C}$ and diluted with diethyl ether $(50 \mathrm{~mL})$. The layers were separated and the organic layer was sequentially washed with saturated aqueous sodium bicarbonate solution $(3 \times 20 \mathrm{~mL})$, saturated aqueous sodium chloride solution $(20 \mathrm{~mL})$ and dried over sodium sulfate. The dried solution was filtered and concentrated. The crude product was purified by flash-column chromatography on silica gel ( $2 \%$ ethyl acetate in cyclohexane) to furnish 2-cyclononen-1-one (S-11, $165 \mathrm{mg}, 56 \%$ ) as a colorless liquid. The obtained analytical data were in full agreement with those reported in literature. ${ }^{6}$

TLC (10\% ethyl acetate in cyclohexane): $R_{f}=0.48(\mathrm{CAM})$.

\section{2-lodo-cyclohexen-1-one (S-12)}

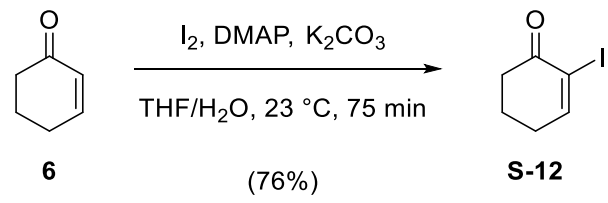

To a solution of 2-cyclohexen-1-one $(6,1.9 \mathrm{~g}, 20 \mathrm{mmol}, 1$ equiv) in tetrahydrofuran (50 $\mathrm{mL})$ and water $(50 \mathrm{~mL})$ was subsequently added potassium carbonate $(3.3 \mathrm{~g}, 24 \mathrm{mmol}$, 1.2 equiv), iodine (10 g, $40 \mathrm{mmol}, 2.0$ equiv) and 4-dimethylaminopyridine ( $0.49 \mathrm{~g}, 4.0 \mathrm{mmol}$, 0.20 equiv) at $23^{\circ} \mathrm{C}$. After 75 minutes, ethyl acetate $(150 \mathrm{~mL})$ was added and the layers were separated. The organic layer was washed with saturated aqueous sodium thiosulfate solution $(2 \times 100 \mathrm{~mL})$, dried over magnesium sulfate, filtered and concentrated. The crude product was purified by flash-column chromatography on silica gel $(20 \%$ diethyl ether in pentane) to furnish iodide S-12 (3.4 g, 76\%) as a yellow solid. The obtained analytical data was in full agreement with those reported in literature. ${ }^{8}$

TLC $\left(25 \%\right.$ ethyl acetate in hexanes): $R_{\mathrm{f}}=0.44\left(\mathrm{UV}, \mathrm{KMnO}_{4}\right)$. 


\section{2-Cyclopropyl-cyclohexen-1-one (S-13)}

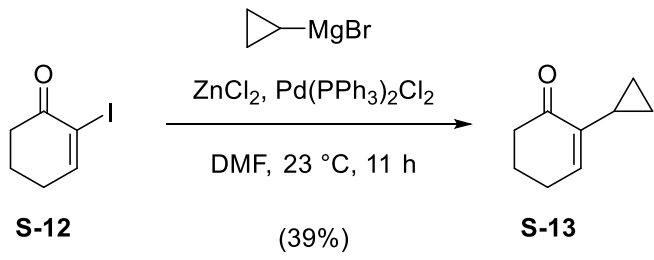

Cyclopropylmagnesium bromide was freshly prepared by the addition of bromocyclopropane $(1.1 \mathrm{~g}, 9.3 \mathrm{mmol}, 1$ equiv) and iodine (catalytic amount) to a suspension of magnesium $\left(0.25 \mathrm{~g}, 10 \mathrm{mmol}, 1.1\right.$ equiv) in tetrahydrofuran $(10 \mathrm{~mL})$ at $23^{\circ} \mathrm{C}$. The reaction mixture was heated to $70{ }^{\circ} \mathrm{C}$. After four hours, the reaction mixture was allowed to cool to $23^{\circ} \mathrm{C}$ and was used in the next step without further purification.

To a solution of zinc chloride $(7.9 \mathrm{~mL}, 7.9 \mathrm{mmol}, 1.1$ equiv, $1.0 \mathrm{M}$ in tetrahydrofuran) was added freshly prepared cyclopropylmagnesium bromide solution $(10 \mathrm{~mL}, 8.7 \mathrm{mmol}$, 1.2 equiv, $0.84 \mathrm{M}$ in tetrahydrofuran) at $23^{\circ} \mathrm{C}$. Upon addition, a solution of iodide $\mathbf{S}-12(1.6 \mathrm{~g}$, $7.2 \mathrm{mmol}, 1$ equiv) and bis(triphenylphosphine)palladium(II) dichloride $(0.25 \mathrm{~g}, 0.36 \mathrm{mmol}$, $5.0 \mathrm{~mol} \%)$ in $\mathrm{N}, \mathrm{N}$-dimethylformamide $(20 \mathrm{~mL})$ was added. After eleven hours, aqueous citric acid solution $(50 \mathrm{~mL}, 5 \%)$ and diethyl ether $(50 \mathrm{~mL})$ were added and the layers were separated. The aqueous layer was extracted with diethyl ether $(2 \times 30 \mathrm{~mL})$, the combined organic layers were dried over magnesium sulfate, filtered and concentrated. The crude product was purified by flash-column chromatography on silica gel (20\% diethyl ether in pentane) to furnish enone S-13 $(0.39 \mathrm{~g}, 39 \%)$ as a colorless oil. The obtained analytical data was in full agreement with those reported in literature. ${ }^{9}$

TLC (20\% diethyl ether in pentane): $R_{\mathrm{f}}=0.36\left(\mathrm{UV}, \mathrm{KMnO}_{4}, \mathrm{CAM}\right)$.

\section{Alcohol S-15}

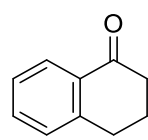

S-14

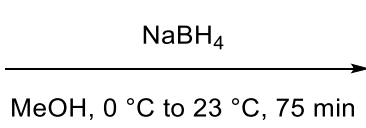

$(96 \%)$

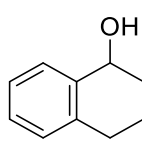

S-15

To a solution of $\alpha$-tetralone (S-14, $2.0 \mathrm{~g}, 14 \mathrm{mmol}, 1$ equiv) in methanol (15 $\mathrm{mL}$ ) was added sodium borohydride $\left(1.3 \mathrm{~g}, 34 \mathrm{mmol}, 2.5\right.$ equiv) at $0^{\circ} \mathrm{C}$. Upon addition, the reaction mixture was allowed to warm to $23^{\circ} \mathrm{C}$. After 75 minutes, water $(50 \mathrm{~mL})$ and diethyl ether $(50 \mathrm{~mL})$ were added and the layers were separated. The aqueous layer was extracted with diethyl ether $(2 \times 50 \mathrm{~mL})$, the combined organic layers were dried over sodium sulfate, filtered and concentrated. The crude product was purified by flash-column chromatography on silica gel 
(20\% ethyl acetate in hexanes) to furnish benzylic alcohol S-15 $(1.9 \mathrm{~g}, 96 \%)$ as a colorless oil. The obtained analytical data was in full agreement with those reported in literature. ${ }^{10}$

TLC $\left(20 \%\right.$ ethyl acetate in hexanes): $R_{\mathrm{f}}=0.24\left(\mathrm{UV}, \mathrm{KMnO}_{4}\right)$.

\section{Enone S-16}

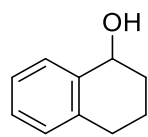

S-15

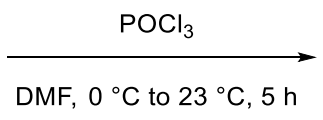

$(76 \%)$

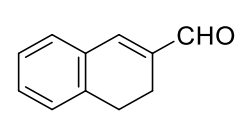

S-16

To a solution of alcohol S-15 $(1.9 \mathrm{~g}, 13 \mathrm{mmol}, 1$ equiv) in $N, N$-dimethylformamide $(5.0 \mathrm{~mL}$, $65 \mathrm{mmol}, 5.0$ equiv) was dropwise added phosphorus(V) oxychloride $(3.1 \mathrm{~mL}, 33 \mathrm{mmol}$, 2.5 equiv) via syringe pump within 45 minutes at $0^{\circ} \mathrm{C}$. Upon addition, the reaction mixture was allowed to warm to $23^{\circ} \mathrm{C}$. After four hours, the reaction mixture was cooled to $0{ }^{\circ} \mathrm{C}$ and water $(10 \mathrm{~mL})$ was carefully added. Aqueous sodium hydroxide solution $(75 \mathrm{~mL}, 10 \%)$ and ethyl acetate $(75 \mathrm{~mL})$ were added and the layers were separated. The aqueous layer was extracted with ethyl acetate $(2 \times 75 \mathrm{~mL})$, the combined organic layers were dried over sodium sulfate, filtered and concentrated. The crude product was purified by flash-column chromatography on silica gel (20\% ethyl acetate in hexanes) to furnish enone S-16 (1.6 g, $76 \%)$ as a colorless oil. The obtained analytical data was in full agreement with those reported in literature. ${ }^{11}$

$\operatorname{TLC}\left(25 \%\right.$ ethyl acetate in hexanes): $R_{\mathrm{f}}=0.51\left(\mathrm{UV}, \mathrm{KMnO}_{4}\right)$.

\section{$N$-(acyloxy)phthalimide S-18}

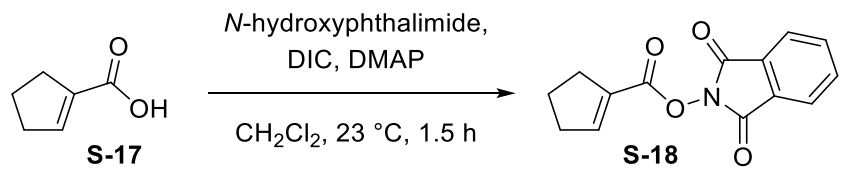

$(82 \%)$

$\mathrm{N}$-(acyloxy)phthalimide S-18 was synthesized based on a procedure described by Baran and coworkers. ${ }^{12}$ To a solution of 1-cyclopentenecarboxylic acid (S-17, $0.50 \mathrm{~g}, 4.5 \mathrm{mmol}$, 1.0 equiv) in dichloromethane $(22 \mathrm{~mL})$ were subsequently added $N$-hydroxyphthalimide ( $0.73 \mathrm{~g}, 4.5 \mathrm{mmol}, 1.0$ equiv) and 4-dimethylaminopyridine (55 mg, $0.45 \mathrm{mmol}, 0.10$ equiv) at $23^{\circ} \mathrm{C}$. Upon addition, $N, N$-diisopropylcarbodiimide $(0.67 \mathrm{~mL}, 4.5 \mathrm{mmol}, 1.0$ equiv) was dropwise added. After 1.5 hours, the reaction mixture was filtered through a plug of Celite ${ }^{\circledR}$ 
and the filtrate was concentrated. The crude product was purified by flash-column chromatography on silica gel $(10 \%$ ethyl acetate in hexanes) to furnish $\mathrm{N}$-(acyloxy)phthalimide S-18 (0.95 g, 82\%) as a colorless solid.

TLC $(10 \%$ ethyl acetate in hexane $): R_{\mathrm{f}}=0.23\left(\mathrm{UV}, \mathrm{KMnO}_{4}\right)$.

${ }^{1} \mathbf{H}$ NMR $\left(\mathrm{CDCl}_{3}, 599 \mathrm{MHz}\right): \delta=7.92-7.88(\mathrm{~m}, 2 \mathrm{H}), 7.81-7.77(\mathrm{~m}, 2 \mathrm{H}), 7.23(\mathrm{tt}, J=2.7$, $1.9 \mathrm{~Hz}, 1 \mathrm{H}), 2.76-2.70(\mathrm{~m}, 2 \mathrm{H}), 2.66-2.61(\mathrm{~m}, 2 \mathrm{H}), 2.05(\mathrm{tt}, J=8.5,7.2 \mathrm{~Hz}, 2 \mathrm{H})$.

${ }^{13} \mathrm{C}$ NMR $\left(\mathrm{CDCl}_{3}, 151 \mathrm{MHz}\right): \delta=162.3,160.7,150.4,134.8,131.3,129.2,124.1,34.3,31.5$, 23.0.

IR (Diamond-ATR, $\left.\mathrm{CDCl}_{3}\right): \tilde{v}_{\max }=1766(m), 1740(v s), 1620(w), 1468(w), 1361(m)$, $1184(m), 1016(w), 994(s), 878(m), 696(s)$.

HRMS (El) calc. for $\mathrm{C}_{14} \mathrm{H}_{11} \mathrm{NO}_{4}[\mathrm{M}]^{+}: 257.0683$; found: 257.0687.

$\mathbf{M P}=113-115^{\circ} \mathrm{C}$

\section{Enone S-21}

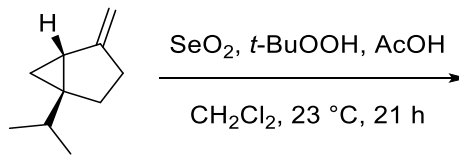

S-19
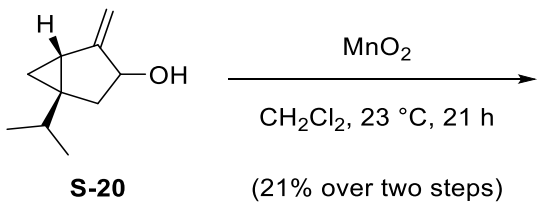

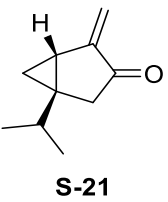

S-21

To a suspension of selenium dioxide (22 $\mathrm{mg}, 0.20 \mathrm{mmol}, 2.0 \mathrm{~mol} \%$ ) in dichloromethane $(5.5 \mathrm{~mL})$ were subsequently added acetic acid $(57 \mu \mathrm{L}, 1.0 \mathrm{mmol}, 0.10$ equiv), sabinene (S-19, $2.2 \mathrm{~mL}, 10 \mathrm{mmol}, 1$ equiv, natural sample of $75 \%$ purity) and a solution of tert-butyl hydroperoxide ( $2.3 \mathrm{~mL}, 12 \mathrm{mmol}, 1.2$ equiv, $5.0 \mathrm{M}$ in decane) at $23^{\circ} \mathrm{C}$. After 21 hours, the reaction mixture was poured into saturated aqueous sodium thiosulfate solution $(50 \mathrm{~mL})$ followed by the addition of water $(50 \mathrm{~mL})$ and dichloromethane $(100 \mathrm{~mL})$. The layers were separated, the aqueous layer was extracted with dichloromethane $(2 \times 50 \mathrm{~mL})$, the combined organic layers were dried over sodium sulfate, filtered and concentrated. The crude product was purified by flash-column chromatography on silica gel ( $20 \%$ diethyl ether in pentane) to furnish allylic alcohol S-20 as a colorless oil $(0.98 \mathrm{~g})$, which was used in the next step without further purification.

TLC $(20 \%$ diethyl ether in pentane $): R_{\mathrm{f}}=0.23\left(\mathrm{CAM}, \mathrm{KMnO}_{4}\right)$. 
To a solution of allylic alcohol S-20 (0.98 g) in dichloromethane (22 $\mathrm{mL}$ ) was added manganese dioxide $\left(2.5 \mathrm{~g}, 29 \mathrm{mmol}, 2.9\right.$ equiv) at $23^{\circ} \mathrm{C}$. After twelve hours, additional manganese dioxide ( $2.5 \mathrm{~g}, 29 \mathrm{mmol}, 2.9$ equiv) was added. After eleven hours, the reaction mixture was filtered through a plug of Celite $^{\circledR}$ and the filtrate was concentrated. The crude product was purified by flash-column chromatography on silica gel ( $2 \%$ diethyl ether in pentane) to furnish enone S-21 (0.24 g, 21\% over two steps) as a colorless, amorphous solid. The obtained analytical data were in full agreement with those reported in literature. ${ }^{13}$

TLC (2\% diethyl ether in pentane): $R_{\mathrm{f}}=0.20\left(\mathrm{UV}, \mathrm{KMnO}_{4}\right)$.

${ }^{1} \mathrm{H}$ NMR $\left(\mathrm{CDCl}_{3}, 400 \mathrm{MHz}\right): \delta=5.75(\mathrm{~s}, 1 \mathrm{H}), 5.25(\mathrm{~s}, 1 \mathrm{H}), 2.50(\mathrm{dt}, J=19.1,2.3 \mathrm{~Hz}, 1 \mathrm{H})$, $2.30(\mathrm{dd}, J=19.1,1.9 \mathrm{~Hz}, 1 \mathrm{H}), 2.05(\mathrm{dd}, J=8.5,3.4 \mathrm{~Hz}, 1 \mathrm{H}), 1.53-1.42(\mathrm{~m}, 1 \mathrm{H}), 1.08-$ $1.03(\mathrm{~m}, 1 \mathrm{H}), 0.98(\mathrm{~d}, J=6.9,1.6 \mathrm{~Hz}, 3 \mathrm{H}), 0.94(\mathrm{dd}, J=6.9,1.6 \mathrm{~Hz}, 3 \mathrm{H}), 0.45-0.40(\mathrm{~m}$, $1 \mathrm{H})$.

${ }^{13} \mathrm{C}$ NMR $\left(\mathrm{CDCl}_{3}, 101 \mathrm{MHz}\right): \delta=206.2,148.2,113.7,41.6,32.9,30.6,26.6,22.0,19.8,19.7$.

IR (Diamond-ATR, $\left.\mathrm{CDCl}_{3}\right): \tilde{v}_{\max }=2959(\mathrm{~m}), 1726$ (vs), $1637(s), 1465(w), 1271(s)$, $1231(w), 1102(s), 917(s), 723(m), 628(s)$.

HRMS (ESI) calc. for $\mathrm{C}_{10} \mathrm{H}_{14} \mathrm{NaO}[\mathrm{M}+\mathrm{Na}]^{+}:$173.0937; found: 173.0934 .

Note: The first oxidation step also led to the formation of side products, which were inseparable from the desired product. This might result from impurities of the natural sample of sabinene (racemic, $75 \%$ purity, purchased from Sigma-Aldrich). Purification was successful after the second oxidation step to obtain enone S-21 analytically pure after flash-column chromatography.

\section{(Z)-benzalacetone (S-23)}

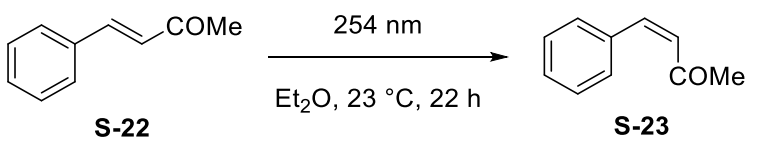

$(33 \%)$

A solution of (E)-benzalacetone (S-22, $1.0 \mathrm{~g}, 4.5 \mathrm{mmol}$, 1 equiv) in diethyl ether (12 $\mathrm{mL})$ in a quartz-tube was irradiated in a Rayonet photochemical reactor (wavelength $=254 \mathrm{~nm}$ ) at $23^{\circ} \mathrm{C}$. After 22 hours, the solvent was removed and the crude product was purified by flashcolumn chromatography on silica gel (10\% diethyl ether in pentane) to furnish (Z)-benzalacetone (S-23, $0.33 \mathrm{~g}, 33 \%)$ as a yellow oil. The obtained analytical data were in full agreement with those reported in literature. ${ }^{14}$

$\operatorname{TLC}(25 \%$ diethyl ether in pentane $): R_{\mathrm{f}}=0.38\left(\mathrm{UV}, \mathrm{KMnO}_{4}\right)$ 


\section{Ester S-26}

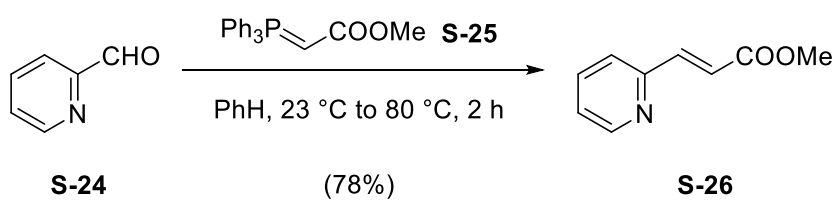

To a solution of aldehyde S-24 $(5.4 \mathrm{~g}, 57 \mathrm{mmol}, 1$ equiv) in benzene (180 $\mathrm{mL}$ ) was slowly added phosphorus ylide S-25 $\left(29 \mathrm{~g}, 85 \mathrm{mmol}, 1.5\right.$ equiv) at $23^{\circ} \mathrm{C}$. Upon addition, the reaction mixture was heated to $80^{\circ} \mathrm{C}$. After two hours, the solvent was removed under reduced pressure and the crude product was purified by flash-column chromatography on silica gel (25\% ethyl acetate in hexanes) to furnish ester S-26 $(7.2 \mathrm{~g}, 78 \%)$ as a yellow solid. The obtained analytical data was in full agreement with those reported in literature. ${ }^{15}$

TLC (25\% ethyl acetate in hexanes): $R_{\mathrm{f}}=0.20\left(\mathrm{UV}, \mathrm{KMnO}_{4}\right)$.

\section{Lactone S-28}

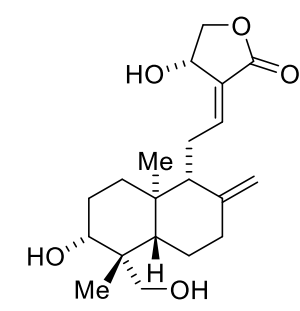

(-)-andrographolide (S-27)

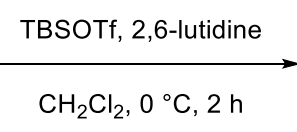

$(72 \%)$

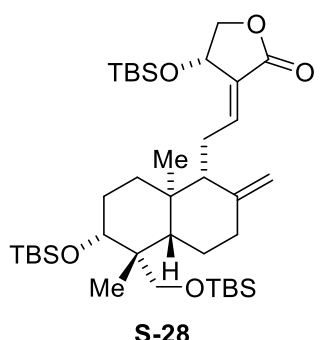

S-28

To a solution of (-)-Andrographolide (S-27, $1.0 \mathrm{~g}, 2.8 \mathrm{mmol}$, 1 equiv) in dichloromethane $(20 \mathrm{~mL})$ was added 2,6-lutidine ( $2.3 \mathrm{~mL}, 14 \mathrm{mmol}, 5.0$ equiv). The mixture was cooled to $0{ }^{\circ} \mathrm{C}$ and tert-butyldimethylsilyl trifluoromethanesulfonate $(2.9 \mathrm{~mL}, 13 \mathrm{mmol}, 4.5$ equiv) was added dropwise. After two hours, water $(100 \mathrm{~mL})$ and ethyl acetate $(100 \mathrm{~mL})$ were added and the layers were separated. The aqueous layer was extracted with ethyl acetate $(2 \times 100 \mathrm{~mL})$, the combined organic layers were washed with saturated aqueous sodium chloride solution $(2 \times 150 \mathrm{~mL})$, dried over sodium sulfate, filtered and concentrated. The crude product was purified by flash-column chromatography on silica gel ( $50 \%$ dichloromethane in pentane) to furnish silyl-protected triol S-28 $(1.4 \mathrm{~g}, 72 \%)$ as a colorless solid. The obtained analytical data were in full agreement with those reported in literature. ${ }^{16}$

$\operatorname{TLC}(50 \%$ dichloromethane in pentane $): R_{\mathrm{f}}=0.20\left(\mathrm{UV}, \mathrm{KMnO}_{4}\right)$. 


\section{3-Vinylpyridine (S-30)}

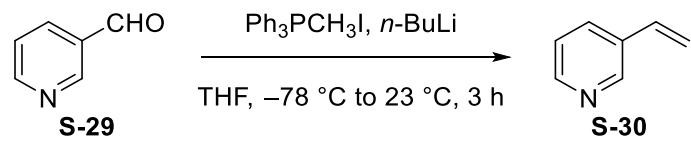

$(23 \%)$

To a solution of methyltriphenylphosphonium iodide $(4.39 \mathrm{~g}, 10.5 \mathrm{mmol}, 1.15$ equiv) in tetrahydrofuran $(55 \mathrm{~mL})$ was added a solution of $n$-butyllithium $(4.26 \mathrm{~mL}, 10.1 \mathrm{mmol}$, 1.10 equiv, $2.36 \mathrm{M}$ in $n$-hexane) dropwise at $-78^{\circ} \mathrm{C}$. Upon addition, the solution was allowed to warm to $0{ }^{\circ} \mathrm{C}$. After 15 minutes, a solution of aldehyde $\mathbf{S}-29(1.00 \mathrm{~g}, 9.15 \mathrm{mmol}$, 1 equiv) in tetrahydrofuran $(8 \mathrm{~mL})$ was slowly added. After 15 minutes, the reaction mixture was allowed to warm to $23^{\circ} \mathrm{C}$. After three hours, water $(100 \mathrm{~mL})$ and diethyl ether $(50 \mathrm{~mL})$ were added, the layers were separated and the aqueous layer was extracted with diethyl ether $(2 \times 100 \mathrm{~mL})$. The combined organic layers were dried over sodium sulfate, filtered and concentrated. The crude product was purified by flash-column chromatography on silica gel (50\% ethyl acetate in cyclohexane) to furnish pyridine S-30 (220 mg, 23\%) as a volatile, yellowish oil. The obtained analytical data was in full agreement with those reported in literature. ${ }^{17}$

$\operatorname{TLC}\left(50 \%\right.$ ethyl acetate in cyclohexane): $R_{\mathrm{f}}=0.29\left(\mathrm{UV}, \mathrm{KMnO}_{4}\right)$.

\section{2-Vinylfuran (S-32)}

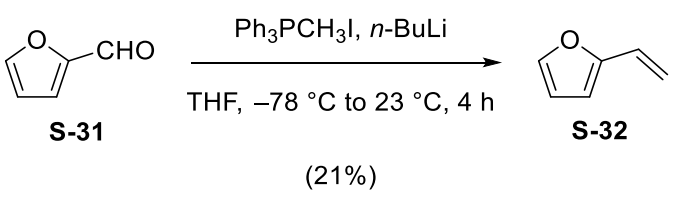

To a solution of methyltriphenylphosphonium iodide $(3.95 \mathrm{~g}, 9.48 \mathrm{mmol}, 1.15$ equiv) in tetrahydrofuran $(50 \mathrm{~mL})$ was dropwise added a solution of $n$-butyllithium $(3.84 \mathrm{~mL}$, $9.10 \mathrm{mmol}$, 1.10 equiv, $2.36 \mathrm{M}$ in $n$-hexane) at $-78{ }^{\circ} \mathrm{C}$. Upon addition, the solution was allowed to warm to $0^{\circ} \mathrm{C}$. After 15 minutes, a solution of freshly distilled aldehyde S-31 (800 mg, 8.24 mmol, 1 equiv) in tetrahydrofuran ( $8 \mathrm{~mL}$ ) was slowly added. After 15 minutes, the reaction mixture was allowed to warm to $23^{\circ} \mathrm{C}$. After three hours, water $(100 \mathrm{~mL})$ and diethyl ether $(50 \mathrm{~mL})$ was added. The layers were separated and the aqueous layer was extracted with diethyl ether $(2 \times 100 \mathrm{~mL})$. The combined organic layers were dried over sodium sulfate, filtered and concentrated. The crude product was purified by flash-column chromatography on silica gel (1\% diethyl ether in pentane) to furnish furan S-32 (162 mg, 
$21 \%)$ as a volatile, yellowish oil. The obtained analytical data was in full agreement with those reported in literature. ${ }^{18}$

TLC ( $1 \%$ diethyl ether in pentane): $R_{\mathrm{f}}=0.61\left(\mathrm{KMnO}_{4}, \mathrm{CAM}\right)$.

\section{Enone S-34}

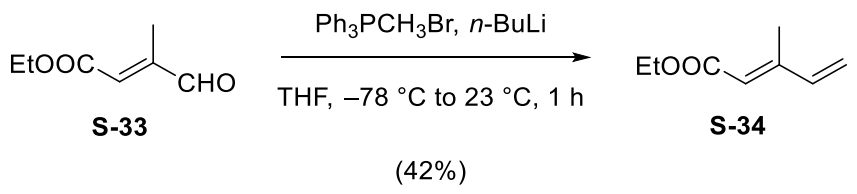

To a solution of methyltriphenylphosphonium bromide $(3.9 \mathrm{~g}, 11 \mathrm{mmol}, 1.1$ equiv) in tetrahydrofuran $(30 \mathrm{~mL})$ was dropwise added a solution of $n$-butyllithium $(5.0 \mathrm{~mL}, 11 \mathrm{mmol}$, 1.1 equiv, $2.2 \mathrm{M}$ in $n$-hexane) at $-78^{\circ} \mathrm{C}$. Upon addition, the solution was allowed to warm to $0{ }^{\circ} \mathrm{C}$. After 1.5 hours, the solution was cooled to $-78^{\circ} \mathrm{C}$ and then a solution of aldehyde S-33 (1.4 g, $10 \mathrm{mmol}, 1$ equiv) in tetrahydrofuran $(14 \mathrm{~mL})$ was slowly added. Upon addition, the reaction mixture was allowed to warm to $23^{\circ} \mathrm{C}$. After one hour, the reaction mixture was poured into water $(100 \mathrm{~mL})$ and diethyl ether $(50 \mathrm{~mL})$ was added. The layers were separated and the aqueous layer was extracted with diethyl ether $(2 \times 75 \mathrm{~mL})$. The combined organic layers were dried over magnesium sulfate, filtered and concentrated. The crude product was purified by flash-column chromatography on silica gel ( $5 \%$ diethyl ether in pentane) to furnish enone S-34 $(0.59 \mathrm{~g}, 42 \%)$ as a volatile, colorless oil. The obtained analytical data was in full agreement with those reported in literature. ${ }^{19}$

TLC (10\% diethyl ether in hexanes): $R_{\mathrm{f}}=0.47\left(\mathrm{UV}, \mathrm{KMnO}_{4}\right)$.

${ }^{1} \mathrm{H}$ NMR $\left(\mathrm{CDCl}_{3}, 400 \mathrm{MHz}\right): \delta=6.40(\mathrm{ddd}, J=17.4,10.6,0.7 \mathrm{~Hz}, 1 \mathrm{H}), 5.83-5.75(\mathrm{~m}, 1 \mathrm{H})$, $5.66-5.55(\mathrm{~m}, 1 \mathrm{H}), 5.42-5.34(\mathrm{~m}, 1 \mathrm{H}), 4.18(\mathrm{q}, J=7.1 \mathrm{~Hz}, 2 \mathrm{H}), 2.27(\mathrm{~d}, J=1.2 \mathrm{~Hz}, 3 \mathrm{H})$, $1.29(\mathrm{t}, J=7.1 \mathrm{~Hz}, 3 \mathrm{H})$.

${ }^{13} \mathrm{C} \mathrm{NMR}\left(\mathrm{CDCl}_{3}, 101 \mathrm{MHz}\right): \delta=167.1,152.1,140.3,120.2,119.5,60.0,14.5,13.2$.

IR (Diamond-ATR, $\left.\mathrm{CDCl}_{3}\right): \tilde{v}_{\max }=1713(s), 1632(w), 1605(m) .1367(w), 1354(w), 1232(s)$, $1156(v s), 1072(w), 1037(w), 871(w)$.

HRMS (EI) calc. for $\mathrm{C}_{8} \mathrm{H}_{12} \mathrm{OS}[\mathrm{M}]^{+}:$140.0832; found: 140.0827 . 


\section{Alkynone S-36}

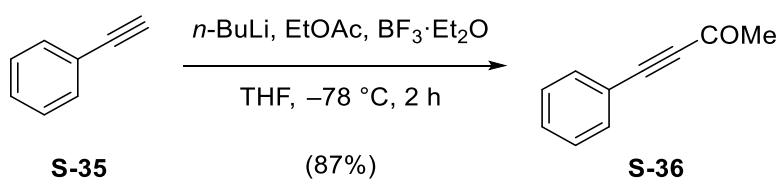

To a solution of ethynylbenzene (S-35, $1.6 \mathrm{~g}, 16 \mathrm{mmol}, 2.0$ equiv) in tetrahydrofuran $(16 \mathrm{~mL})$ under argon was added dropwise a solution of $n$-butyllithium $(6.7 \mathrm{~mL}, 16 \mathrm{mmol}, 2.0$ equiv, $2.50 \mathrm{M}$ in $n$-hexane) at $-78^{\circ} \mathrm{C}$. After one hour, ethyl acetate $(0.78 \mathrm{~mL}, 8.0 \mathrm{mmol}, 1$ equiv $)$ and boron trifluoride etherate $(2.4 \mathrm{~mL}, 19 \mathrm{mmol}, 2.4$ equiv, $47 \%$ in diethyl ether) were subsequently added. After 40 minutes, saturated aqueous solution of ammonium chloride $(20 \mathrm{~mL})$ and ethyl acetate $(20 \mathrm{~mL})$ were added and the layers were separated. The aqueous layer was extracted with ethyl acetate $(2 \times 15 \mathrm{~mL})$, the combined organic layers were dried over sodium sulfate, filtered and concentrated. The crude product was purified by flashcolumn chromatography on silica gel $(30 \%$ to $50 \%$ dichloromethane in pentane) to furnish alkynone S-36 $(1.0 \mathrm{~g}, 87 \%)$ as an orange oil. The obtained analytical data were in full agreement with those reported in literature. ${ }^{20}$

TLC $\left(10 \%\right.$ ethyl acetate in cyclohexane): $R_{\mathrm{f}}=0.5\left(\mathrm{UV}, \mathrm{KMnO}_{4}\right)$.

\section{Benzyl Ester S-38}

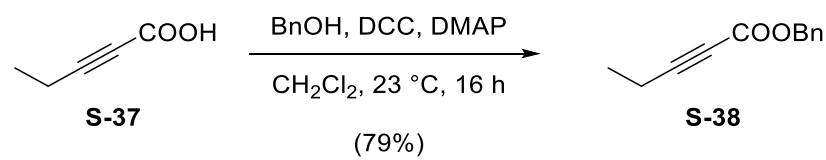

To a solution of hex-3-ynoic acid (S-37, $0.56 \mathrm{~g}, 5.0 \mathrm{mmol}, 1$ equiv) in dichloromethane $(50 \mathrm{~mL})$ was added benzyl alcohol $(0.52 \mathrm{~mL}, 5.0 \mathrm{mmol}, 1.0$ equiv) followed by 4-dimethylaminopyridine (37 mg, $0.30 \mathrm{mmol}, 6.0 \mathrm{~mol} \%)$ and dicyclohexylcarbodiimide $(1.0 \mathrm{~g}$, $5.0 \mathrm{mmol}, 1.0$ equiv) at $0{ }^{\circ} \mathrm{C}$. The solution was allowed to warm to $23^{\circ} \mathrm{C}$. After 17 hours, the reaction mixture was filtered through a plug of Celite ${ }^{\circledR}$ and the filtrate was concentrated. The crude product was purified by flash-column chromatography on silica gel (10\% diethyl ether in cyclohexane) to furnish benzyl ester S-38 $(0.80 \mathrm{~g}, 79 \%)$ as a yellow oil.

TLC (10\% diethyl ether in cyclohexane): $\mathrm{R}_{f}=0.58\left(\mathrm{UV}, \mathrm{KMnO}_{4}\right)$.

${ }^{1} \mathbf{H}$ NMR $\left(\mathrm{CDCl}_{3}, 400 \mathrm{MHz}\right) \delta=7.40-7.31(\mathrm{~m}, 5 \mathrm{H}), 5.19(\mathrm{~s}, 2 \mathrm{H}), 2.34(\mathrm{q}, J=7.5 \mathrm{~Hz}, 2 \mathrm{H})$, $1.20(\mathrm{t}, J=7.5 \mathrm{~Hz}, 3 \mathrm{H})$. 
${ }^{13} \mathrm{C}$ NMR $\left(\mathrm{CDCl}_{3}, 101 \mathrm{MHz}\right) \delta=153.8,135.1,128.7,128.6,128.6,91.2,72.4,67.5,12.6$, 12.5 .

IR (Diamond-ATR, $\mathrm{CDCl}_{3}$ ): $\tilde{v}_{\max }=2983(w), 2236(\mathrm{~m}), 1704$ (vs), 1234 (vs), 1079 (s), $1051(s), 748(s), 696(s), 608(m), 566(m)$.

HRMS (ESI) calc. for $\mathrm{C}_{12} \mathrm{H}_{12} \mathrm{NaO}_{2}[\mathrm{M}+\mathrm{Na}]^{+}: 211.0730$; found: 211.0727.

\section{Dibromide S-40}

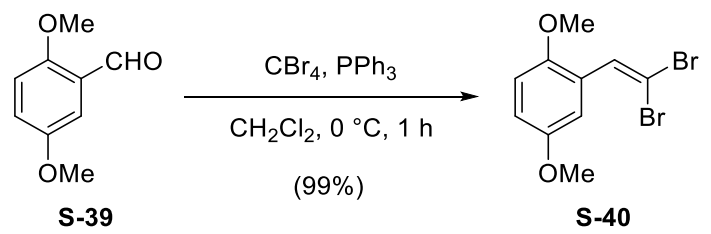

Triphenylphosphine $(10.5 \mathrm{~g}, 40.0 \mathrm{mmol}, 4.00$ equiv) and tetrabromomethane $(6.63 \mathrm{~g}$, $20.0 \mathrm{mmol}, 2.00$ equiv) were dissolved in dichloromethane $(50 \mathrm{~mL})$ at $0{ }^{\circ} \mathrm{C}$. After 40 minutes, a solution of 2,5-dimethoxybenzaldehyde (S-39, $1.66 \mathrm{~g}, 10.0 \mathrm{mmol}, 1$ equiv) in dichloromethane $(30 \mathrm{~mL})$ was added dropwise. After 20 minutes, water $(200 \mathrm{~mL})$ and dichloromethane $(50 \mathrm{~mL})$ were added, the layers were separated, and the aqueous layer was extracted with dichloromethane $(3 \times 200 \mathrm{~mL})$. The combined organic layers were dried over sodium sulfate, filtered and concentrated. The crude product was purified by flash-column chromatography on silica gel (10\% diethyl ether in pentane) to furnish dibromide $\mathbf{S}-40$ $(3.20 \mathrm{~g}, 99 \%)$ as a colorless solid. The obtained analytical data were in full agreement with those reported in literature. ${ }^{21}$

TLC (10\% diethyl ether in pentane): $R_{\mathrm{f}}=0.58\left(\mathrm{UV}, \mathrm{KMnO}_{4}\right)$. 


\section{Methyl Propiolate S-41}

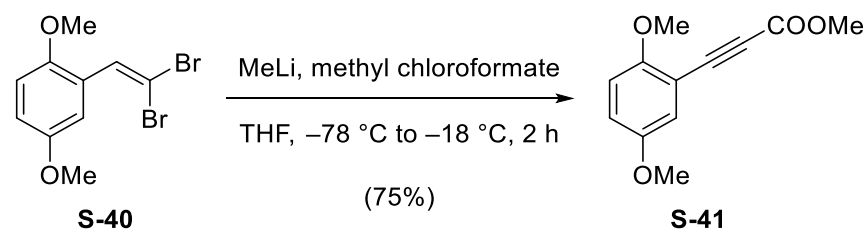

To a solution of dibromide S-40 (2.81 g, $8.74 \mathrm{mmol}, 1$ equiv) in tetrahydrofuran $(90 \mathrm{~mL})$ was added methyl lithium (16.0 mL, $26.2 \mathrm{mmol}, 3.00$ equiv, $1.60 \mathrm{M}$ in $n$-hexane) at $-78{ }^{\circ} \mathrm{C}$. After one hour, methyl chloroformate $(4.06 \mathrm{~mL}, 52.4 \mathrm{mmol}, 6.00$ equiv) was added. The reaction mixture was allowed to warm to $-18{ }^{\circ} \mathrm{C}$. After one hour, water $(30 \mathrm{~mL})$ and diethyl ether $(30 \mathrm{~mL})$ were added, the layers were separated, and the aqueous layer was extracted with diethyl ether $(2 \times 30 \mathrm{~mL})$. The combined organic layers were dried over sodium sulfate, filtered and concentrated. The crude product was purified by flash-column chromatography on silica gel (20\% to $30 \%$ diethyl ether in pentane) to furnish methyl propiolate S-41 (1.45 g, $75 \%)$ as a pale-yellow solid.

TLC (10\% diethyl ether in pentane): $\mathrm{R}_{f}=0.18\left(\mathrm{UV}, \mathrm{KMnO}_{4}\right)$.

${ }^{1} \mathbf{H}$ NMR $\left(\mathrm{CDCl}_{3}, 400 \mathrm{MHz}\right) \delta=7.03(\mathrm{~d}, J=3.1 \mathrm{~Hz}, 1 \mathrm{H}), 6.96(\mathrm{dd}, J=9.1,3.1 \mathrm{~Hz}, 1 \mathrm{H}), 6.82$ (d, $J=9.2 \mathrm{~Hz}, 1 \mathrm{H}), 3.84(\mathrm{~s}, 3 \mathrm{H}), 3.82(\mathrm{~s}, 3 \mathrm{H}), 3.75(\mathrm{~s}, 3 \mathrm{H})$.

${ }^{13} \mathrm{C}$ NMR $\left(\mathrm{CDCl}_{3}, 101 \mathrm{MHz}\right) \delta=156.3,154.6,153.2,119.1,118.9,112.3,109.0,84.2,83.6$, $56.5,55.9,52.8$.

IR (Diamond-ATR, $\mathrm{CDCl}_{3}$ ): $\tilde{v}_{\max }=2953(w), 2837(w), 2212(s), 1704(s), 1499(s), 1222(v s)$, $1158(s), 1040(s), 747(m), 712(m)$.

HRMS (ESI) calc. for $\mathrm{C}_{12} \mathrm{H}_{12} \mathrm{NaO}_{4}[\mathrm{M}+\mathrm{Na}]^{+}:$243.0633; found: 243.0623.

MP: $71-73^{\circ} \mathrm{C}$. 


\subsection{General Procedures}

\subsubsection{Optimization}

Table 1 | Optimization of the reaction conditions for high-pressure and thermal conditions. ${ }^{a}$

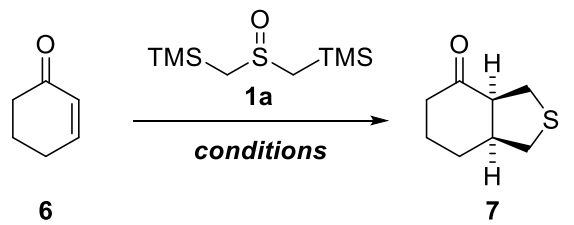

\begin{tabular}{|c|c|c|c|c|c|}
\hline Entry & Solvent & Pressure [kbar] & Temp. $\left[{ }^{\circ} \mathrm{C}\right]$ & Time [h] & Yield of $7[\%]^{b}$ \\
\hline 1 & n-hexane & 14 & 23 & 2 & 0 \\
\hline 2 & $\mathrm{CH}_{2} \mathrm{Cl}_{2}$ & 14 & 23 & 2 & 0 \\
\hline 3 & $\mathrm{CH}_{3} \mathrm{CN}$ & 14 & 23 & 2 & 0 \\
\hline 4 & NMP & 14 & 23 & 2 & 0 \\
\hline 5 & 1,4-dioxane & 14 & 23 & 2 & 31 \\
\hline 6 & $\mathrm{Et}_{2} \mathrm{O}$ & 14 & 23 & 2 & 33 \\
\hline 7 & THF & 14 & 23 & 2 & 41 \\
\hline 8 & DMF & 14 & 23 & 2 & 43 \\
\hline 9 & DMSO & 14 & 23 & 2 & 54 \\
\hline 10 & DMAC & 14 & 23 & 2 & 59 \\
\hline 11 & DMPU & 14 & 23 & 2 & 86 \\
\hline 12 & DMPU & 10 & 23 & 2 & 79 \\
\hline 13 & DMPU & 5 & 23 & 2 & 85 \\
\hline 14 & DMPU & 2 & 23 & 2 & 72 \\
\hline 15 & DMPU & 1 & 23 & 2 & 62 \\
\hline 16 & DMPU & 0.001 & 23 & 24 & 16 \\
\hline 17 & DMPU & 0.001 & 60 & 1 & 30 \\
\hline 18 & DMPU & 0.001 & 80 & 1 & 40 \\
\hline 19 & DMPU & 0.001 & 80 & 2 & 38 \\
\hline 20 & DMPU & 0.001 & 100 & 1 & 29 \\
\hline
\end{tabular}

a Reaction was performed on $0.50 \mathrm{mmol}$ scale by using 2.0 equivalents of sulfoxide 1a $(0.15 \mathrm{M}$ in $N, N$ 'dimethylpropylenurea for high-pressure conditions or $0.50 \mathrm{M}$ for thermal conditions). $\mathrm{b}$ Isolated yield after purification by flash-column chromatography on silica gel.

\subsubsection{General Procedure 1: High-Pressure (GP1)}

The dipolarophile $(0.50 \mathrm{mmol}, 1$ equiv) was placed in a PTFE-vial (max. volume $=10 \mathrm{~mL}$ ) and dry $N, N$ '-dimethylpropylenurea $(2.0 \mathrm{~mL})$ was added. A solution of sulfoxide $1 \mathrm{a}(0.22 \mathrm{~g}$, $1.0 \mathrm{mmol}, 2.0$ equiv) in $N^{\prime} N^{\prime}$-dimethylpropylenurea $(1.2 \mathrm{~mL})$ was added and the vial was sealed with a PTFE-cap. The vial was carefully lowered into the oil-filled compression 
chamber (Figure 1, A1-A2) and high-pressure (5 kbar, A3-A5) was applied. After two hours, the reaction mixture was depressurized to atmospheric pressure (A6) and removed from the compression chamber. The reaction mixture was poured into water $(7 \mathrm{~mL})$, diethyl ether $(7 \mathrm{~mL})$ was added and the layers were separated. The aqueous layer was extracted with diethyl ether $(2 \times 7 \mathrm{~mL})$, the combined organic layers were washed with water $(3 \times 10 \mathrm{~mL})$, dried over sodium sulfate, filtered and concentrated. Conversion was evaluated by ${ }^{1} \mathrm{H}$ NMR spectroscopy of the crude mixture which was subsequently purified by flash-column chromatography on silica gel.
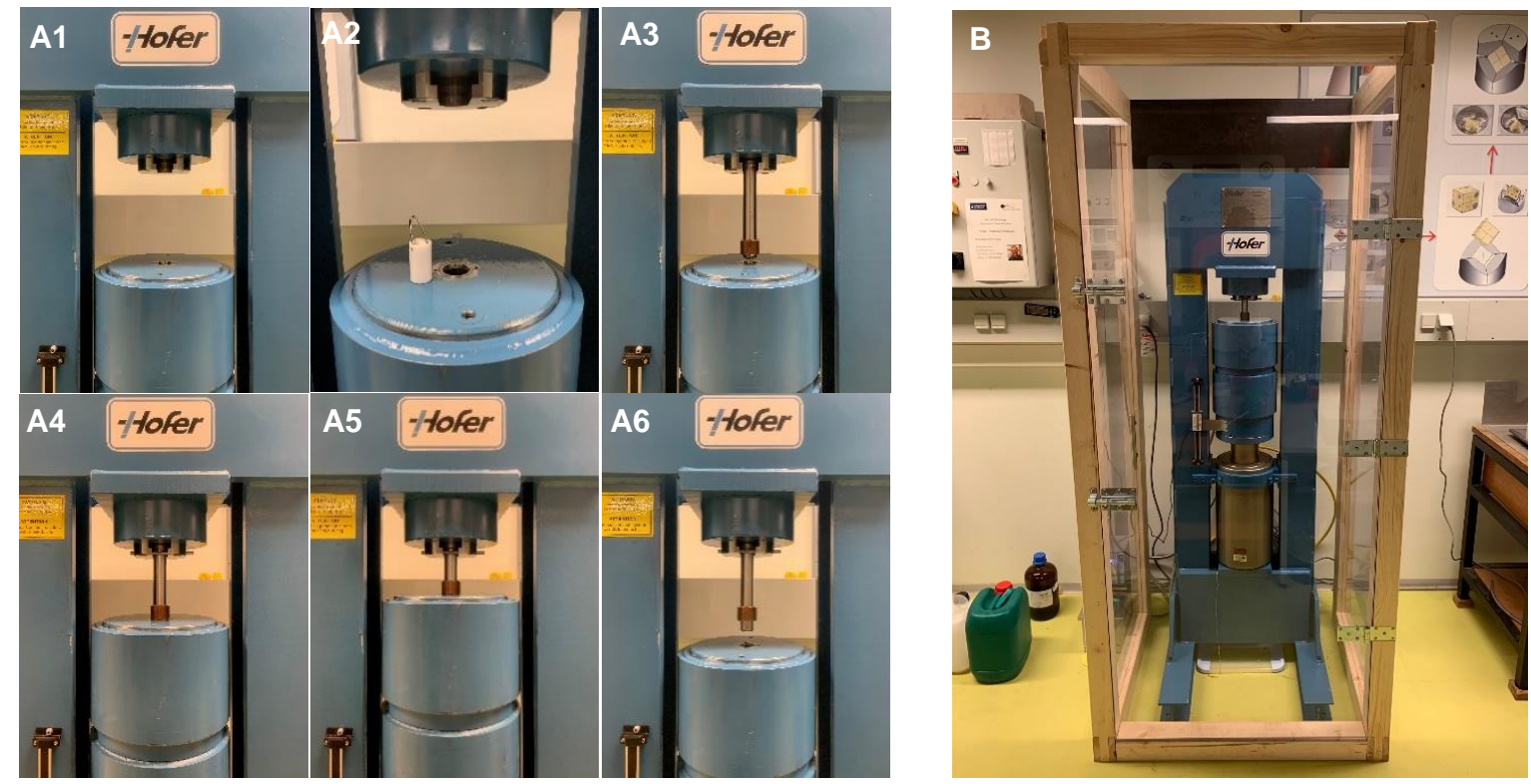

Figure 1: A) Loading of the compression chamber (A1) with the reaction mixture, placed in a custom-made PTFE reaction-vial (A2) and application of high pressure (A3-A6). B) Emulsion high-pressure machine at 5 kbar locked in a custom-made protection-housing.

\subsubsection{General Procedure 2: Heat (GP2)}

The dipolarophile ( $0.50 \mathrm{mmol}, 1$ equiv) was placed in an oven-dried vial under argon and dry $N, N^{\prime}$-dimethylpropylenurea $(0.5 \mathrm{~mL})$ was added. The mixture was heated to $80^{\circ} \mathrm{C}$ in an aluminum heating block. After ten minutes, a solution of sulfoxide 1a $(0.22 \mathrm{~g}, 1.0 \mathrm{mmol}$, 2.0 equiv) in $N, N^{\prime}$-dimethylpropylenurea $(0.5 \mathrm{~mL})$ was dropwise added and the reaction mixture was kept at $80^{\circ} \mathrm{C}$. After one hour, the reaction mixture was allowed to cool to $23^{\circ} \mathrm{C}$. Then the reaction mixture was poured into water $(7 \mathrm{~mL})$, diethyl ether $(7 \mathrm{~mL})$ was added and the layers were separated. The aqueous layer was extracted with diethyl ether $(2 \times 7 \mathrm{~mL})$, the combined organic layers were washed with water $(3 \times 10 \mathrm{~mL})$, dried over sodium sulfate, filtered and concentrated. Conversion was evaluated by ${ }^{1} \mathrm{H}$ NMR spectroscopy of the crude mixture which was purified by flash-column chromatography on silica gel. 


\subsection{Substrate Scope}

\subsection{1 $\alpha, \beta-$-Unsaturated Systems}

\section{Tetrahydrothiophene 7}

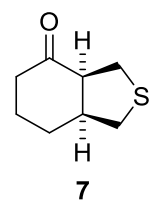

Following the General Procedures GP1 and GP2, 2-cyclohexen-1-one (6, $48 \mathrm{mg}, 0.50 \mathrm{mmol})$ was converted into tetrahydrothiophene 7. Purification by flash-column chromatography on silica gel (30\% diethyl ether in pentane) furnished 7 (GP1: $66 \mathrm{mg}, 85 \%$; GP2: $31 \mathrm{mg}, 40 \%$ ) as a pale-yellow oil.

TLC (50\% diethyl ether in pentane): $R_{\mathrm{f}}=0.41\left(\mathrm{UV}, \mathrm{KMnO}_{4}, \mathrm{CAM}\right)$.

${ }^{1} \mathbf{H}$ NMR $\left(\mathrm{CDCl}_{3}, 400 \mathrm{MHz}\right): \delta=3.30(\mathrm{dd}, J=10.3,5.8 \mathrm{~Hz}, 1 \mathrm{H}), 3.00-2.92(\mathrm{~m}, 1 \mathrm{H}), 2.92-$ $2.88(\mathrm{~m}, 1 \mathrm{H}), 2.88-2.79(\mathrm{~m}, 2 \mathrm{H}), 2.68(\mathrm{dd}, J=10.1,6.1 \mathrm{~Hz}, 1 \mathrm{H}), 2.50-2.41(\mathrm{~m}, 1 \mathrm{H}), 2.37-$ $2.28(\mathrm{~m}, 1 \mathrm{H}), 2.04-1.77(\mathrm{~m}, 4 \mathrm{H})$.

${ }^{13} \mathrm{C}$ NMR $\left(\mathrm{CDCl}_{3}, 101 \mathrm{MHz}\right): \delta=210.1,56.5,47.3,39.6,35.4,30.9,25.8,24.1$.

IR (Diamond-ATR, $\left.\mathrm{CDCl}_{3}\right)$ : $\tilde{v}_{\max }=2933(s), 1706(v s), 1449(w), 1358(w), 1260(w)$, $1228(w), 1143(w), 1103(w), 863(w), 519(w)$.

HRMS (EI) calc. for $\mathrm{C}_{8} \mathrm{H}_{12} \mathrm{OS}[\mathrm{M}]^{+}:$156.0603; found: 156.0603 .

\section{Tetrahydrothiophene 8a}

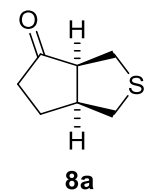

Following the General Procedures GP1 and GP2, 2-cyclopenten-1-one (41 mg, $0.50 \mathrm{mmol}$ ) was converted into tetrahydrothiophene 8a. Purification by flash-column chromatography on silica gel (25\% diethyl ether in pentane) furnished 8a (GP1: 49 mg, 69\%; GP2: $37 \mathrm{mg}, 52 \%$ ) as a colorless oil.

TLC (40\% diethyl ether in pentane): $R_{\mathrm{f}}=0.33\left(\mathrm{KMnO}_{4}\right)$. 
${ }^{1} \mathrm{H}$ NMR $\left(\mathrm{CDCl}_{3}, 400 \mathrm{MHz}\right): \delta=3.21-3.06(\mathrm{~m}, 3 \mathrm{H}), 3.00(\mathrm{dd}, J=11.4,8.6 \mathrm{~Hz}, 1 \mathrm{H}), 2.90(\mathrm{td}$, $J=8.3,3.0 \mathrm{~Hz}, 1 \mathrm{H}), 2.66(\mathrm{dd}, J=11.4,4.6 \mathrm{~Hz}, 1 \mathrm{H}), 2.34(\mathrm{t}, J=8.2 \mathrm{~Hz}, 2 \mathrm{H}), 2.28-2.13(\mathrm{~m}$, $1 \mathrm{H}), 1.93-1.79(\mathrm{~m}, 1 \mathrm{H})$.

${ }^{13} \mathrm{C} \mathrm{NMR}\left(\mathrm{CDCl}_{3}, 101 \mathrm{MHz}\right): \delta=219.0,56.2,45.2,38.6,37.3,33.9,25.9$.

IR (Diamond-ATR, $\left.\mathrm{CDCl}_{3}\right): \tilde{v}_{\max }=2933(w), 1736(v s), 1448(w), 1406(w), 1252(w)$, $1208(w), 1171(w), 1112(w), 855(w), 707(w)$.

HRMS (El) calc. for $\mathrm{C}_{7} \mathrm{H}_{10} \mathrm{OS}[\mathrm{M}]^{+}:$142.0447; found: 142.0445.

\section{Tetrahydrothiophene $8 b$}

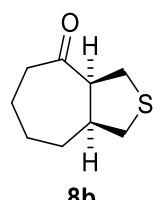

Following the General Procedures GP1 and GP2, 2-cyclohepten-1-one (29, $55 \mathrm{mg}$, $0.50 \mathrm{mmol}$ ) was converted into tetrahydrothiophene $\mathbf{8 b}$. Purification by flash-column chromatography on silica gel (dichloromethane) furnished 8b (GP1: $65 \mathrm{mg}, 77 \%$; GP2: $21 \mathrm{mg}, 25 \%$ ) as a colorless oil.

TLC (dichloromethane): $R_{\mathrm{f}}=0.32\left(\mathrm{KMnO}_{4}\right)$.

${ }^{1} \mathrm{H}$ NMR $\left(\mathrm{CDCl}_{3}, 400 \mathrm{MHz}\right): \delta=3.32-3.23(\mathrm{~m}, 1 \mathrm{H}), 3.16(\mathrm{dd}, J=11.5,8.9 \mathrm{~Hz}, 1 \mathrm{H}), 3.09$ (dd, $J=11.2,6.3 \mathrm{~Hz}, 1 \mathrm{H}), 2.99(\mathrm{dd}, J=11.5,7.9 \mathrm{~Hz}, 1 \mathrm{H}), 2.81-2.72(\mathrm{~m}, 1 \mathrm{H}), 2.63$ (ddd, $J=$ 12.6, 10.9, 3.3 Hz, 1H), 2.56 (dd, $J=11.2,5.1 \mathrm{~Hz}, 1 \mathrm{H}), 2.50-2.41(\mathrm{~m}, 1 \mathrm{H}), 2.02-1.91$ (m, $1 \mathrm{H}), 1.91-1.82(\mathrm{~m}, 1 \mathrm{H}), 1.78-1.67(\mathrm{~m}, 1 \mathrm{H}), 1.65-1.47(\mathrm{~m}, 2 \mathrm{H}), 1.46-1.33(\mathrm{~m}, 1 \mathrm{H})$.

${ }^{13} \mathrm{C} \mathrm{NMR}\left(\mathrm{CDCl}_{3}, 101 \mathrm{MHz}\right): \delta=212.0,59.1,44.6,43.6,39.9,32.3,31.3,28.3,25.5$.

IR (Diamond-ATR, $\left.\mathrm{CDCl}_{3}\right)$ : $\tilde{v}_{\max }=2925(m), 2856(m), 1698(v s), 1445(m), 1315(w)$, $1160(w), 1129(w), 983(w), 844(w), 718(w)$.

HRMS (EI) calc. for $\mathrm{C}_{9} \mathrm{H}_{14} \mathrm{OS}[\mathrm{M}]^{+}:$170.0760; found: 170.0757. 


\section{Tetrahydrothiophene 8c}

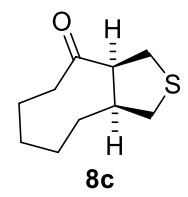

Following the General Procedures GP1 (14 kbar) and GP2, 2-cycloocten-1-one (S-8, 62 mg, $0.50 \mathrm{mmol}$ ) was converted into tetrahydrothiophene $8 \mathrm{c}$. Purification by flash-column chromatography on silica gel (2\% ethyl acetate in toluene) furnished 8c (GP1: $72 \mathrm{mg}, 78 \%$; GP2: $18 \mathrm{mg}, 20 \%$ ) as a colorless liquid.

TLC $\left(10 \%\right.$ ethyl acetate in cyclohexane): $R_{\mathrm{f}}=0.35\left(\mathrm{UV}, \mathrm{KMnO}_{4}\right)$.

${ }^{1} \mathrm{H}$ NMR $\left(\mathrm{CDCl}_{3}, 400 \mathrm{MHz}\right): \delta=3.14(\mathrm{td}, J=8.0,5.1 \mathrm{~Hz}, 1 \mathrm{H}), 3.10-3.01(\mathrm{~m}, 2 \mathrm{H}), 3.01-$ $2.93(\mathrm{~m}, 2 \mathrm{H}), 2.64$ (ddd, $J=12.8,11.1,3.8 \mathrm{~Hz}, 1 \mathrm{H}), 2.59-2.52(\mathrm{~m}, 1 \mathrm{H}), 2.31$ (ddd, $J=12.8$, 7.0, 3.7 Hz, 1H), 1.89 (ddq, $J=13.8,6.9,3.5 \mathrm{~Hz}, 1 \mathrm{H}), 1.82-1.42(\mathrm{~m}, 6 \mathrm{H}), 1.26-1.13(\mathrm{~m}$, $1 \mathrm{H})$.

${ }^{13} \mathrm{C} \mathrm{NMR}\left(\mathrm{CDCl}_{3}, 101 \mathrm{MHz}\right): \delta=214.5,58.6,43.5,42.8,38.5,29.9,29.8,28.1,26.9,24.7$.

IR (Diamond-ATR, CDCl $)$ ): $\tilde{v}_{\max }=2925(s), 2856(m), 1696(s), 1455(m), 1333(w), 1180(w)$, $1102(w), 1037(w), 899(w), 703(w)$.

HRMS (ESI) calc. for $\mathrm{C}_{10} \mathrm{H}_{16} \mathrm{NaOS}[\mathrm{M}+\mathrm{Na}]^{+}:$207.0820; found: 207.0807 .

\section{Tetrahydrothiophene 8d}

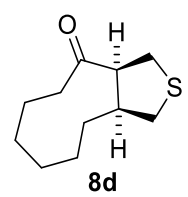

Following the General Procedures GP1 (14 kbar) and GP2, 2-cyclononen-1-one (S-11, $69 \mathrm{mg}, 0.50 \mathrm{mmol}$ ) was converted into tetrahydrothiophene $8 \mathrm{~d}$. Purification by flash-column chromatography on silica gel (3\% ethyl acetate in toluene) furnished $8 \mathbf{d}$ (GP1: $73 \mathrm{mg}, 74 \%$; GP2: $18 \mathrm{mg}, 18 \%$ ) as a colorless liquid.

TLC (10\% ethyl acetate in cyclohexane): $R_{\mathrm{f}}=0.27\left(\mathrm{UV}, \mathrm{KMnO}_{4}\right)$. 
${ }^{1} \mathrm{H}$ NMR $\left(\mathrm{CDCl}_{3}, 400 \mathrm{MHz}\right): \delta=3.16-3.06(\mathrm{~m}, 3 \mathrm{H}), 3.01-2.93(\mathrm{~m}, 1 \mathrm{H}), 2.88-2.77(\mathrm{~m}$, 2H), 2.59 (dd, $J=10.3,2.2 \mathrm{~Hz}, 1 \mathrm{H}), 2.25$ (ddd, $J=14.1,6.5,3.9 \mathrm{~Hz}, 1 \mathrm{H}), 1.92-1.80$ (m, $1 \mathrm{H}), 1.74-1.61(\mathrm{~m}, 1 \mathrm{H}), 1.56-1.30(\mathrm{~m}, 8 \mathrm{H})$.

${ }^{13} \mathrm{C}$ NMR $\left(\mathrm{CDCl}_{3}, 101 \mathrm{MHz}\right): \delta=212.9,61.2,42.8,40.8,40.2,29.2,27.8,24.7,24.1,23.7$, 23.5.

IR (Diamond-ATR, $\left.\mathrm{CDCl}_{3}\right): \tilde{v}_{\max }=2926(s), 2870(m), 1698(s), 1462(m), 1356(m)$, $1287(w), 1247(w), 1142(m), 1037(m), 713(m)$.

HRMS (ESI) calc. for $\mathrm{C}_{11} \mathrm{H}_{18} \mathrm{NaOS}[\mathrm{M}+\mathrm{Na}]^{+}: 221.0976$; found: 221.0963.

\section{Tetrahydrothiophene $8 \mathrm{e}$}

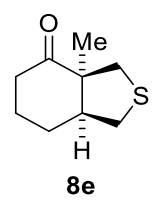

Following the General Procedures GP1 and GP2, 2-methyl-2-cyclohexen-1-one (55 mg, $0.50 \mathrm{mmol}$ ) was converted into tetrahydrothiophene 8e. Purification by flash-column chromatography on silica gel (20\% ethyl acetate in hexanes) furnished $8 \mathrm{e}$ (GP1: $43 \mathrm{mg}$, 52\%; GP2: $6.5 \mathrm{mg}, 8 \%$ ) as a colorless oil.

$\operatorname{TLC}\left(30 \%\right.$ ethyl acetate in hexanes): $R_{\mathrm{f}}=0.44\left(\mathrm{KMnO}_{4}\right)$.

${ }^{1} \mathrm{H}$ NMR $\left(\mathrm{CDCl}_{3}, 599 \mathrm{MHz}\right): \delta=3.36(\mathrm{~d}, J=10.6 \mathrm{~Hz}, 1 \mathrm{H}), 3.06(\mathrm{dd}, J=10.8,6.4 \mathrm{~Hz}, 1 \mathrm{H})$, $2.76(\mathrm{dd}, J=10.8,4.3 \mathrm{~Hz}, 1 \mathrm{H}), 2.54(\mathrm{~d}, J=10.6 \mathrm{~Hz}, 1 \mathrm{H}), 2.52-2.40(\mathrm{~m}, 2 \mathrm{H}), 2.39-2.35(\mathrm{~m}$, $1 \mathrm{H}), 2.05-1.95(\mathrm{~m}, 1 \mathrm{H}), 1.86-1.74(\mathrm{~m}, 3 \mathrm{H}), 1.25(\mathrm{~s}, 3 \mathrm{H})$.

${ }^{13} \mathrm{C} \mathrm{NMR}\left(\mathrm{CDCl}_{3}, 151 \mathrm{MHz}\right): \delta=212.3,58.8,52.9,39.3,37.8,36.4,26.5,24.4,21.5$.

IR (Diamond-ATR, $\left.\mathrm{CDCl}_{3}\right): \tilde{v}_{\max }=2936(m), 2864(w), 1703(v s), 1454(m), 1374(w)$, $1270(w), 1107(w), 991(w), 932(w), 838(w)$.

HRMS (EI) calc. for $\mathrm{C}_{9} \mathrm{H}_{14} \mathrm{OS}[\mathrm{M}]^{+}:$170.0760; found: 170.0757 . 


\section{Tetrahydrothiophene $8 f$}

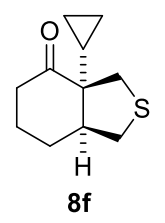

Following the General Procedures GP1 (14 kbar) and GP2, 2-cyclopropyl-cyclohexen-1-one (S-13, $72 \mathrm{mg}, 0.50 \mathrm{mmol}$ ) was converted into tetrahydrothiophene $\mathbf{8 f}$. Purification by flashcolumn chromatography on silica gel (toluene) furnished 8f (GP1: 49 mg, 50\%; GP2: not detected) as a colorless solid.

$\operatorname{TLC}\left(5 \%\right.$ ethyl acetate in toluene): $R_{\mathrm{f}}=0.46\left(\mathrm{KMnO}_{4}\right)$.

${ }^{1} \mathrm{H}$ NMR $\left(\mathrm{CDCl}_{3}, 400 \mathrm{MHz}\right): \delta=3.21(\mathrm{~d}, J=10.9 \mathrm{~Hz}, 1 \mathrm{H}), 2.90(\mathrm{dd}, J=10.9,7.5 \mathrm{~Hz}, 1 \mathrm{H})$, $2.75(\mathrm{dd}, J=11.0,9.4 \mathrm{~Hz}, 1 \mathrm{H}), 2.68-2.53(\mathrm{~m}, 2 \mathrm{H}), 2.37$ (dtd, $J=15.1,4.7,1.3 \mathrm{~Hz}, 1 \mathrm{H}$ ), $2.22(\mathrm{~d}, J=10.9 \mathrm{~Hz}, 1 \mathrm{H}$ ), 2.11 (dddd, $J=13.9,8.9,6.0,4.6 \mathrm{~Hz}, 1 \mathrm{H}), 2.01-1.89(\mathrm{~m}, 2 \mathrm{H})$, $1.84-1.75(\mathrm{~m}, 1 \mathrm{H}), 1.17-1.09(\mathrm{~m}, 1 \mathrm{H}), 0.55-0.41(\mathrm{~m}, 3 \mathrm{H}), 0.20(\mathrm{dtd}, J=8.0,5.5,3.7 \mathrm{~Hz}$, $1 \mathrm{H})$.

${ }^{13} \mathrm{C}$ NMR $\left(\mathrm{CDCl}_{3}, 101 \mathrm{MHz}\right): \delta=211.0,61.4,51.1,38.2,34.5,32.6,24.5,22.4,16.6,3.0,1.8$.

IR (Diamond-ATR, $\left.\mathrm{CDCl}_{3}\right): \tilde{v}_{\max }=2939(\mathrm{~s}), 2863(\mathrm{~m}), 1705(\mathrm{vs}), 1452(\mathrm{~m}), 1427(w)$, $1314(w), 1251(w), 1140(w), 1021(w), 830(w)$.

HRMS (ESI) calc. for $\mathrm{C}_{11} \mathrm{H}_{16} \mathrm{NaOS}[\mathrm{M}+\mathrm{Na}]^{+}: 219.0814$; found: 219.0811.

$M P=36-38^{\circ} \mathrm{C}$.

\section{Tetrahydrothiophenes (+)-8g and (+)-S-42}

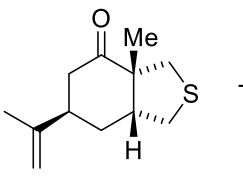

$(+)-8 g$

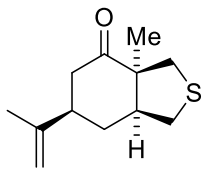

$(+)-S-42$

Following the General Procedures GP1 (14 kbar) and GP2, (R)-(-)-carvone (75 mg, $0.50 \mathrm{mmol}$ ) was converted into tetrahydrothiophenes (+)-8g and (+)-S-42. Purification by flash-column chromatography on silica gel (dichloromethane) furnished (+)-8g (GP1: $31 \mathrm{mg}$, 31\%; GP2: $9.8 \mathrm{mg}, 9 \%$ ) as a colorless, crystalline solid and (+)-S-42 (GP1: $31 \mathrm{mg}, 31 \%$; GP2: $7.6 \mathrm{mg}, 7 \%$ ) as a colorless, amorphous solid. Recrystallization of the major diastereomer $(+)-\mathbf{8 g}$ from dichloromethane gave crystals suitable for $\mathrm{X}$-ray analysis. 
Major diastereomer (+)-8g:

TLC (dichloromethane): $R_{\mathrm{f}}=0.33\left(\mathrm{KMnO}_{4}\right)$.

${ }^{1} \mathbf{H}$ NMR $\left(\mathrm{CDCl}_{3}, 400 \mathrm{MHz}\right): \delta=4.88-4.79(\mathrm{~m}, 1 \mathrm{H}), 4.77-4.68(\mathrm{~m}, 1 \mathrm{H}), 3.54(\mathrm{~d}, J=$ $10.7 \mathrm{~Hz}, 1 \mathrm{H}), 2.97(\mathrm{dd}, J=11.0,7.2 \mathrm{~Hz}, 1 \mathrm{H}), 2.76(\mathrm{dd}, J=11.0,8.2 \mathrm{~Hz}, 1 \mathrm{H}), 2.72-2.63(\mathrm{~m}$, $1 \mathrm{H}), 2.60-2.47(\mathrm{~m}, 3 \mathrm{H}), 2.49-2.42(\mathrm{~m}, 1 \mathrm{H}), 1.96(\mathrm{dd}, J=6.9,5.1 \mathrm{~Hz}, 2 \mathrm{H}), 1.76(\mathrm{~s}, 3 \mathrm{H})$, $1.31(\mathrm{~s}, 3 \mathrm{H})$.

${ }^{13} \mathrm{C}$ NMR $\left(\mathrm{CDCl}_{3}, 101 \mathrm{MHz}\right): \delta=211.4,146.8,111.1,58.3,50.0,42.3,41.1,38.5,35.2,29.3$, 22.8, 21.2 .

IR (Diamond-ATR, $\left.\mathrm{CDCl}_{3}\right): \tilde{v}_{\max }=2958(w), 2926(m), 1701(v s), 1644(w), 1453(m)$, $1423(w), 1116(w), 1011(w), 892(m), 743(w)$.

HRMS (EI) calc. for $\mathrm{C}_{12} \mathrm{H}_{18} \mathrm{OS}[\mathrm{M}]^{+}:$210.1073; found: 210.1072 .

$\left[\alpha_{D}^{20}\right]=+53.1\left(c=0.97, \mathrm{CH}_{2} \mathrm{Cl}_{2}\right)$.

MP $=67-69^{\circ} \mathrm{C}$.

Minor diastereomer (+)-S-42:

TLC (dichloromethane): $R_{\mathrm{f}}=0.32\left(\mathrm{KMnO}_{4}\right)$.

${ }^{1} \mathbf{H}$ NMR $\left(\mathrm{CDCl}_{3}, 599 \mathrm{MHz}\right): \delta=4.78(\mathrm{ddt}, J=1.9,1.4,0.5 \mathrm{~Hz}, 1 \mathrm{H}), 4.75-4.73(\mathrm{~m}, J=$ $0.8 \mathrm{~Hz}, 1 \mathrm{H}$ ), 3.22-3.16 (m, 2H), 2.77 (dd, $J=10.8,0.9 \mathrm{~Hz}, 1 \mathrm{H}$ ), 2.57 (dd, $J=10.7,0.9 \mathrm{~Hz}$, $1 \mathrm{H}$ ), 2.51 (ddd, $J=14.4,13.5,0.7 \mathrm{~Hz}, 1 \mathrm{H}$ ), 2.42 (ddq, $J=14.6,3.5,1.1 \mathrm{~Hz}, 1 \mathrm{H}$ ), $2.39-2.33$ $(\mathrm{m}, 2 \mathrm{H}), 1.77-1.72(\mathrm{~m}, 5 \mathrm{H}), 1.19(\mathrm{~s}, J=0.8 \mathrm{~Hz}, 3 \mathrm{H})$.

${ }^{13} \mathrm{C}$ NMR $\left(\mathrm{CDCl}_{3}, 150 \mathrm{MHz}\right): \delta=211.9,146.9,110.1,58.0,52.5,44.0,42.5,39.7,37.7,33.1$, $20.3,20.2$.

IR (Diamond-ATR, $\left.\mathrm{CDCl}_{3}\right): \tilde{v}_{\max }=2939(\mathrm{~m}), 1702(\mathrm{vs}), 1645(w), 1459(\mathrm{~m}), 1375(\mathrm{~m})$, $1278(m), 1044(w), 1108(m), 891(s), 696(w)$.

HRMS (EI) calc. for $\mathrm{C}_{12} \mathrm{H}_{18} \mathrm{OS}[\mathrm{M}]^{+}:$210.1073; found: 210.1075 .

$\left[\alpha_{D}^{20}\right]=+169.0\left(c=1.33, \mathrm{CH}_{2} \mathrm{Cl}_{2}\right)$. 


\section{Tetrahydrothiophene $8 \mathrm{~h}$}

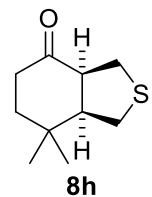

Following the General Procedures GP1 (14 kbar) and GP2, 3,3-dimethyl-2-cyclohexen-1-one (62 $\mathrm{mg}, 0.50 \mathrm{mmol}$ ) was converted into tetrahydrothiophene $8 \mathrm{~h}$. Purification by flash-column chromatography on silica gel (dichloromethane) furnished 8h (GP1: $41 \mathrm{mg}, 45 \%$, GP2: not detected) as a colorless, crystalline solid. Recrystallization from chloroform gave crystals which were suitable for X-ray analysis.

TLC (dichloromethane): $R_{\mathrm{f}}=0.37\left(\mathrm{KMnO}_{4}\right)$.

${ }^{1} \mathbf{H}$ NMR $\left(\mathrm{CDCl}_{3}, 599 \mathrm{MHz}\right): \delta=3.54(\mathrm{dd}, J=10.4,1.1 \mathrm{~Hz}, 1 \mathrm{H}), 3.20$ (ddd, $J=6.4,5.7$, $1.0 \mathrm{~Hz}, 1 \mathrm{H}), 2.79$ (dd, J = 9.9, $5.9 \mathrm{~Hz}, 1 \mathrm{H}), 2.69$ (dd, $J=10.5,6.4 \mathrm{~Hz}, 1 \mathrm{H}), 2.53-2.39(\mathrm{~m}$, 3H), 2.33 (ddd, $J=14.7,4.7,2.4 \mathrm{~Hz}, 1 \mathrm{H}$ ), 1.90 (td, $J=14.0,4.8 \mathrm{~Hz}, 1 \mathrm{H}$ ), 1.67 (ddt, $J=13.9$, 6.5, $2.2 \mathrm{~Hz}, 1 \mathrm{H}), 1.37$ (s, 3H), $1.04(\mathrm{~s}, 3 \mathrm{H})$.

${ }^{13} \mathrm{C}$ NMR $\left(\mathrm{CDCl}_{3}, 151 \mathrm{MHz}\right): \delta=208.5,58.5,52.57,37.7,35.4,32.7,31.0,29.3,28.6,28.6$.

IR (Diamond-ATR, $\mathrm{CDCl}_{3}$ ): $\tilde{v}_{\max }=2961$ (s), 2930 (s), $2870(\mathrm{~m}), 1710$ (vs), $1470(w)$, $1433(w), 1367(m), 1238(m), 1151(w), 1112(s)$.

HRMS (EI) calc. for $\mathrm{C}_{10} \mathrm{H}_{16} \mathrm{OS}$ [M]': 184.0916; found: 184.0915 .

MP $=96-98^{\circ} \mathrm{C}$

\section{Tetrahydrothiophene $8 \mathbf{i}$}

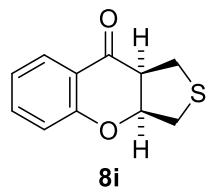

Following the General Procedures GP1 (14 kbar) and GP2, chromone ( $73 \mathrm{mg}, 0.50 \mathrm{mmol}$ ) was converted into tetrahydrothiophene $\mathbf{8 i}$. Purification by flash-column chromatography on silica gel (10\% ethyl acetate in hexanes) furnished $8 \mathbf{i}$ (GP1: $31 \mathrm{mg}, 30 \%$; GP2: not detected) as a colorless, crystalline solid. Recrystallization from dichloromethane gave crystals which were suitable for X-ray analysis.

$\operatorname{TLC}\left(50 \%\right.$ ethyl acetate in hexanes): $R_{\mathrm{f}}=0.56\left(\mathrm{UV}, \mathrm{KMnO}_{4}\right)$. 
${ }^{1} \mathrm{H}$ NMR $\left(\mathrm{CDCl}_{3}, 400 \mathrm{MHz}\right): \delta=7.89(\mathrm{dd}, J=7.9,1.8 \mathrm{~Hz}, 1 \mathrm{H}), 7.51$ (ddd, $J=8.4,7.2,1.8 \mathrm{~Hz}$, $1 \mathrm{H}), 7.04$ (ddd, $J=8.0,7.2,1.1 \mathrm{~Hz}, 1 \mathrm{H}), 6.99(\mathrm{dd}, J=8.4,1.0 \mathrm{~Hz}, 1 \mathrm{H}), 5.23(\mathrm{q}, J=3.5 \mathrm{~Hz}$, $1 \mathrm{H}), 3.31(\mathrm{~d}, J=3.4 \mathrm{~Hz}, 2 \mathrm{H}), 3.21-3.15(\mathrm{~m}, 2 \mathrm{H}), 3.08$ (ddd, $J=10.7,7.3,3.7 \mathrm{~Hz}, 1 \mathrm{H})$.

${ }^{13} \mathrm{C} \mathrm{NMR}\left(\mathrm{CDCl}_{3}, 101 \mathrm{MHz}\right): \delta=191.5,159.9,136.7,127.3,122.1,119.1,118.4,83.7,54.0$, 37.3, 31.0.

IR (Diamond-ATR, $\left.\mathrm{CDCl}_{3}\right): \tilde{v}_{\max }=1683(s), 1607(s), 1473(w), 1462(s), 1306(s), 1221(m)$, $1148(w), 1028(w), 768(m), 752(w)$.

HRMS (EI) calc. for $\mathrm{C}_{11} \mathrm{H}_{10} \mathrm{O}_{2} \mathrm{~S}$ [M] $]^{+}: 206.0396$; found: 206.0396 .

$M P=131-133^{\circ} \mathrm{C}$.

\section{Tetrahydrothiophene $8 \mathbf{j}$}

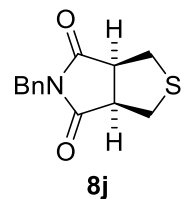

Following the General Procedures GP1 and GP2, N-benzylmaleimide (94 mg, $0.50 \mathrm{mmol}$ ) was converted into tetrahydrothiophene $\mathbf{8 j}$. Purification by flash-column chromatography on silica gel (30\% ethyl acetate in hexanes) furnished 8j (GP1: 106 mg, 85\%; GP2: 34 mg, 27\%) as a pale-yellow solid.

$\operatorname{TLC}\left(50 \%\right.$ ethyl actetate in hexanes): $R_{\mathrm{f}}=0.42\left(\mathrm{KMnO}_{4}\right)$.

${ }^{1} \mathrm{H}$ NMR $\left(\mathrm{CDCl}_{3}, 400 \mathrm{MHz}\right): \delta=7.43-7.17(\mathrm{~m}, 5 \mathrm{H}), 4.68(\mathrm{~s}, 2 \mathrm{H}), 3.51-3.42(\mathrm{~m}, 2 \mathrm{H}), 3.33$ (d, $J=12.7 \mathrm{~Hz}, 2 \mathrm{H}), 3.10-2.98(\mathrm{~m}, 2 \mathrm{H})$.

${ }^{13} \mathrm{C} \mathrm{NMR}\left(\mathrm{CDCl}_{3}, 101 \mathrm{MHz}\right): \delta=177.9,135.5,128.8,128.4,128.0,48.6,43.1,36.2$.

IR (Diamond-ATR, $\left.\mathrm{CDCl}_{3}\right): \tilde{v}_{\max }=2931(w), 1694(v s), 1427(m), 1396(s), 1341(s)$, $1310(m), 1178(m), 1079(w), 975(m), 700(m)$.

HRMS (EI) calc. for $\mathrm{C}_{13} \mathrm{H}_{13} \mathrm{NO}_{2} \mathrm{~S}[\mathrm{M}]^{+}:$247.0662; found: 247.0659 .

MP $=80-82^{\circ} \mathrm{C}$.

Note: Due to its low solubility in $N, N^{\prime}$-dimethylpropylenurea at ambient temperature $\left(23^{\circ} \mathrm{C}\right)$, a mixture of $N, N$ '-dimethylpropylenurea and dichloromethane (30:1) was used for GP1. 


\section{Tetrahydrothiophene (+)-8k}

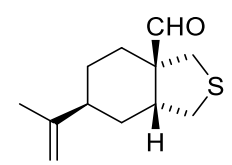

$(+)-8 k$

Following the General Procedures GP1 and GP2, (S)-(-)-perillaldehyde (75 mg, $0.50 \mathrm{mmol}$ ) was converted into tetrahydrothiophene $(+)-\mathbf{8 k}$. Purification by flash-column chromatography on silica gel (10\% dichloromethane in pentane) furnished (+)-8k (GP1: 55 mg, 52\%; GP2: $8.2 \mathrm{mg}, 8 \%)$ as a colorless solid.

TLC $(20 \%$ dichloromethane in pentane $): R_{\mathrm{f}}=0.40\left(\mathrm{KMnO}_{4}\right)$.

${ }^{1} \mathrm{H}$ NMR $\left(\mathrm{CDCl}_{3}, 400 \mathrm{MHz}\right): \delta=9.52(\mathrm{~d}, J=1.1 \mathrm{~Hz}, 1 \mathrm{H}), 4.73-4.69(\mathrm{~m}, 1 \mathrm{H}), 4.69-4.65(\mathrm{~m}$, $1 \mathrm{H}), 3.14-2.96(\mathrm{~m}, 3 \mathrm{H}), 2.86-2.76(\mathrm{~m}, 1 \mathrm{H}), 2.55(\mathrm{~d}, J=10.9 \mathrm{~Hz}, 1 \mathrm{H}), 2.18-2.09(\mathrm{~m}, 1 \mathrm{H})$, 2.01 (dtd, $J=14.7,4.5,3.9,1.4 \mathrm{~Hz}, 1 \mathrm{H}), 1.81-1.69(\mathrm{~m}, 3 \mathrm{H}), 1.68(\mathrm{~s}, 3 \mathrm{H}), 1.48$ (ddd, $J=$ $14.0,12.2,4.7 \mathrm{~Hz}, 1 \mathrm{H}), 1.35-1.24(\mathrm{~m}, 1 \mathrm{H})$.

${ }^{13} \mathrm{C}$ NMR $\left(\mathrm{CDCl}_{3}, 101 \mathrm{MHz}\right): \delta=203.0,148.9,109.5,58.7,44.1,38.3,38.3,34.2,30.1,27.9$, 26.1, 21.0.

IR (Diamond-ATR, $\left.\mathrm{CDCl}_{3}\right)$ : $\tilde{v}_{\max }=2930(\mathrm{~m}), 2859(\mathrm{~m}), 1724(\mathrm{vs}), 1644(\mathrm{~m}), 1454(\mathrm{~m})$, $1376(w), 1230(w), 1083(w), 953(w), 892(w)$.

HRMS (EI) calc. for $\mathrm{C}_{12} \mathrm{H}_{18} \mathrm{OS}[\mathrm{M}]^{+}:$210.1073; found: 210.1067 .

$\left[\boldsymbol{\alpha}_{D}^{\mathbf{2 0}}\right]=+103.3\left(c=3.19, \mathrm{CH}_{2} \mathrm{Cl}_{2}\right)$.

MP $=37-39^{\circ} \mathrm{C}$.

Tetrahydrothiophene (-)-8I

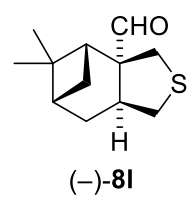

Following the General Procedures GP1 and GP2, $(R)-(-)$-myrtenal (75 mg, $0.50 \mathrm{mmol}$ ) was converted into tetrahydrothiophene (-)-8I. Purification by flash-column chromatography on silica gel (5\% ethyl acetate in hexanes) furnished (-)-8I (GP1: $63 \mathrm{mg}, 60 \%$; GP2: $37 \mathrm{mg}$, $35 \%$ ) as a pale-yellow oil. 
TLC (5\% ethyl acetate in hexanes): $R_{\mathrm{f}}=0.30\left(\mathrm{UV}, \mathrm{KMnO}_{4}\right)$.

${ }^{1} \mathrm{H}$ NMR $\left(\mathrm{CDCl}_{3}, 400 \mathrm{MHz}\right): \delta=9.65(\mathrm{~s}, 1 \mathrm{H}), 3.34-3.23(\mathrm{~m}, 1 \mathrm{H}), 3.19-3.09(\mathrm{~m}, 1 \mathrm{H}), 2.90$ (d, $J=12.9 \mathrm{~Hz}, 1 \mathrm{H}$ ), 2.80 (dd, $J=13.0,1.4 \mathrm{~Hz}, 1 \mathrm{H}$ ), 2.75 (dd, $J=11.7,9.7 \mathrm{~Hz}, 1 \mathrm{H}$ ), 2.37 (dd, $J=6.4,4.9 \mathrm{~Hz}, 1 \mathrm{H}), 2.31-2.18(\mathrm{~m}, 2 \mathrm{H}), 1.95-1.90(\mathrm{~m}, 1 \mathrm{H}), 1.68(\mathrm{dt}, J=13.8,3.3 \mathrm{~Hz}, 1 \mathrm{H})$, $1.34(\mathrm{~d}, J=10.5 \mathrm{~Hz}, 1 \mathrm{H}), 1.24(\mathrm{~s}, 3 \mathrm{H}), 0.69(\mathrm{~s}, 3 \mathrm{H})$.

${ }^{13} \mathrm{C} \mathrm{NMR}\left(\mathrm{CDCl}_{3}, 101 \mathrm{MHz}\right): \delta=204.7,64.8,47.3,45.7,40.6,40.5,38.7,36.3,32.2,26.5$, 26.3, 23.5 .

IR (Diamond-ATR, $\left.\mathrm{CDCl}_{3}\right): \tilde{v}_{\max }=2919(\mathrm{~s}), 2868(\mathrm{~m}), 1724(\mathrm{vs}), 1475(\mathrm{~m}), 1458(\mathrm{~m})$, $1386(w), 1368(w), 1240(w), 1212(w), 853(w)$.

HRMS (El) calc. for $\mathrm{C}_{12} \mathrm{H}_{18} \mathrm{OS}[\mathrm{M}]^{+}:$210.1073; found: 210.1067.

$\left[\boldsymbol{\alpha}_{D}^{\mathbf{2 0}}\right]=-103.2\left(c=3.35, \mathrm{CH}_{2} \mathrm{Cl}_{2}\right)$.

\section{Tetrahydrothiophene $8 \mathrm{~m}$}

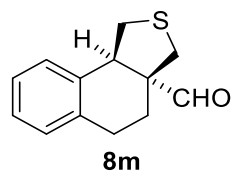

Following the General Procedures GP1 and GP2, enone S-16 $(79 \mathrm{mg}, 0.50 \mathrm{mmol}$ ) was converted into tetrahydrothiophene $8 \mathrm{~m}$. Purification by flash-column chromatography on silica gel (15\% diethyl ether in pentane) furnished 8m (GP1: 95 mg, 87\%; GP2: 58 mg, 53\%) as a pale-yellow, amorphous solid.

$\operatorname{TLC}\left(30 \%\right.$ ethyl acetate in hexanes): $R_{\mathrm{f}}=0.44\left(\mathrm{UV}, \mathrm{KMnO}_{4}\right)$.

${ }^{1} \mathrm{H}$ NMR $\left(\mathrm{CDCl}_{3}, 400 \mathrm{MHz}\right): \delta=9.58(\mathrm{~d}, J=0.9 \mathrm{~Hz}, 1 \mathrm{H}), 7.22-7.13(\mathrm{~m}, 3 \mathrm{H}), 7.12-7.08(\mathrm{~m}$, $1 \mathrm{H}), 3.74(\mathrm{dd}, J=9.8,7.7 \mathrm{~Hz}, 1 \mathrm{H}), 3.34(\mathrm{dd}, J=11.1,7.7 \mathrm{~Hz}, 1 \mathrm{H}), 3.28(\mathrm{~d}, J=11.4 \mathrm{~Hz}, 1 \mathrm{H})$, $2.96-2.84(\mathrm{~m}, 3 \mathrm{H}), 2.79(\mathrm{~d}, J=11.4 \mathrm{~Hz}, 1 \mathrm{H}), 2.20-2.08(\mathrm{~m}, 1 \mathrm{H}), 2.07-1.95(\mathrm{~m}, 1 \mathrm{H})$.

${ }^{13} \mathrm{C} \mathrm{NMR}\left(\mathrm{CDCl}_{3}, 101 \mathrm{MHz}\right): \delta=201.9,135.2,134.9,129.2,129.2,127.1,126.5,58.6,48.2$, $39.5,37.9,26.2,24.8$.

IR (Diamond-ATR, $\left.\mathrm{CDCl}_{3}\right): \tilde{v}_{\max }=2929(w), 2856(w), 2710(w), 1721(v s), 1491(m)$, $1444(m), 1201(w), 962(w), 776(s), 743(s)$.

HRMS (EI) calc. for $\mathrm{C}_{13} \mathrm{H}_{14} \mathrm{OS}[\mathrm{M}]^{+}:$218.0760; found: 218.0755 . 


\section{Tetrahydrothiophene $8 \mathrm{n}$}

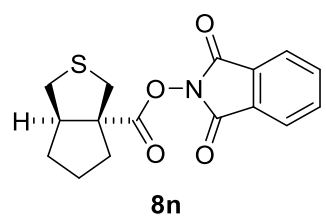

Following the General Procedures GP1 and GP2, N-(acyloxy)phthalimide S-18 (124 mg, $0.50 \mathrm{mmol}$ ) was converted into tetrahydrothiophene $8 \mathrm{n}$. Purification by flash-column chromatography on silica gel (10\% dichloromethane in pentane) furnished 8n (GP1: $132 \mathrm{mg}$, 83\%; GP2: 126 mg, 79\%) as a colorless solid. Recrystallization from dichloromethane gave crystals suitable for X-ray analysis.

TLC (50\% dichloromethane in hexanes): $R_{\mathrm{f}}=0.35\left(\mathrm{UV}, \mathrm{KMnO}_{4}\right)$.

${ }^{1} \mathrm{H}$ NMR $\left(\mathrm{CDCl}_{3}, 400 \mathrm{MHz}\right): \delta=7.89(\mathrm{dd}, J=5.5,3.1 \mathrm{~Hz}, 2 \mathrm{H}), 7.79(\mathrm{dd}, J=5.5,3.1 \mathrm{~Hz}, 2 \mathrm{H})$, $3.50(\mathrm{~d}, J=12.2 \mathrm{~Hz}, 1 \mathrm{H}$ ), 3.37 (qd, $J=7.6,2.7 \mathrm{~Hz}, 1 \mathrm{H}$ ), $3.12(\mathrm{dd}, J=11.8,6.8 \mathrm{~Hz}, 1 \mathrm{H}$ ), 2.85 (d, $J=12.2 \mathrm{~Hz}, 1 \mathrm{H}), 2.63(\mathrm{dd}, J=11.9,2.7 \mathrm{~Hz}, 1 \mathrm{H}), 2.60-2.52(\mathrm{~m}, 1 \mathrm{H}), 2.14-2.04(\mathrm{~m}$, $1 \mathrm{H}), 1.86$ (ddt, $J=12.0,10.0,4.4 \mathrm{~Hz}, 2 \mathrm{H}), 1.76-1.65(\mathrm{~m}, 1 \mathrm{H}), 1.64-1.56(\mathrm{~m}, 1 \mathrm{H})$.

${ }^{13} \mathrm{C}$ NMR $\left(\mathrm{CDCl}_{3}, 101 \mathrm{MHz}\right): \delta=173.0,162.2,134.9,129.1,124.1,63.0,54.0,41.9,39.1$, $38.3,33.5,26.4$.

IR (Diamond-ATR, $\left.\mathrm{CDCl}_{3}\right): \tilde{v}_{\max }=2955(w), 1782(s), 1738(v s), 1467(m), 1368(m)$, $1186(m), 1080(m), 974(s), 878(s), 696(s)$.

HRMS (EI) calc. for $\mathrm{C}_{16} \mathrm{H}_{15} \mathrm{NO}_{4} \mathrm{~S}\left[\mathrm{M}^{+}:\right.$: 317.0716; found: 317.0716 .

$M P=81-83^{\circ} \mathrm{C}$. 


\section{Tetrahydrothiophene 80}

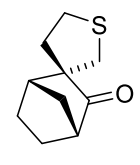

80

Following the General Procedures GP1 and GP2, 3-methylene-2-norbornanone $(61 \mathrm{mg}$, $0.50 \mathrm{mmol}$ ) was converted into tetrahydrothiophene 80 . Purification by flash-column chromatography on silica gel (10\% diethyl ether in pentane) furnished 80 (GP1: 79 mg, 87\%; GP2: $49 \mathrm{mg}, 54 \%$ ) as a colorless, amorphous solid.

TLC $\left(20 \%\right.$ diethyl ether in pentane): $R_{\mathrm{f}}=0.40\left(\mathrm{UV}, \mathrm{KMnO}_{4}\right)$

${ }^{1} \mathrm{H}$ NMR $\left(\mathrm{CDCl}_{3}, 400 \mathrm{MHz}\right): \delta=3.02$ (ddd, $\left.J=10.7,6.3,4.6 \mathrm{~Hz}, 1 \mathrm{H}\right), 2.86(\mathrm{~d}, J=10.7 \mathrm{~Hz}$, $1 \mathrm{H}), 2.81-2.68(\mathrm{~m}, 2 \mathrm{H}), 2.66(\mathrm{dt}, J=5.2,1.5 \mathrm{~Hz}, 1 \mathrm{H}), 2.51(\mathrm{dt}, J=3.2,1.6 \mathrm{~Hz}, 1 \mathrm{H}), 2.05-$ $1.98(\mathrm{~m}, 2 \mathrm{H}), 1.95-1.86(\mathrm{~m}, 2 \mathrm{H}), 1.74-1.60$ (m, 3H), 1.51 (dddd, $J=13.0,8.3,5.9,2.0 \mathrm{~Hz}$, $1 \mathrm{H})$.

${ }^{13} \mathrm{C} \mathrm{NMR}\left(\mathrm{CDCl}_{3}, 101 \mathrm{MHz}\right): \delta=219.4,61.7,50.1,42.6,38.8,36.7,36.2,30.8,24.9,23.5$.

IR (Diamond-ATR, $\left.\mathrm{CDCl}_{3}\right): \tilde{v}_{\max }=2959(s), 1737(\mathrm{vs}), 1455(\mathrm{~m}), 1444(\mathrm{~m}), 1218(w)$, $1194(w), 1078(m), 952(w), 938(w), 772(m)$.

HRMS (EI) calc. for $\mathrm{C}_{10} \mathrm{H}_{14} \mathrm{OS}$ [M]': 182.0760; found: 182.0755 .

\section{Tetrahydrothiophene $8 p$}

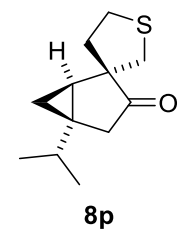

Following the General Procedures GP1 (14 kbar) and GP2, enone S-21 (75 mg, $0.50 \mathrm{mmol}$ ) was converted into tetrahydrothiophene $8 \mathrm{p}$. Purification by flash-column chromatography on silica gel (2\% diethyl ether in pentane) furnished 8p (GP1: $82 \mathrm{mg}, 78 \%$; GP2: $42 \mathrm{mg}, 40 \%$ ) as a colorless solid. Recrystallization from dichloromethane gave crystals suitable for X-ray analysis.

$\operatorname{TLC}\left(5 \%\right.$ diethyl ether in pentane): $R_{\mathrm{f}}=0.24\left(\mathrm{UV}, \mathrm{KMnO}_{4}\right)$. 
${ }^{1} \mathrm{H}$ NMR $\left(\mathrm{CDCl}_{3}, 400 \mathrm{MHz}\right): \delta=3.04$ (ddd, $\left.J=10.4,8.0,2.5 \mathrm{~Hz}, 1 \mathrm{H}\right), 2.94(\mathrm{td}, J=10.6$, $6.3 \mathrm{~Hz}, 1 \mathrm{H}), 2.82(\mathrm{~d}, J=10.2 \mathrm{~Hz}, 1 \mathrm{H}), 2.79-2.72(\mathrm{~m}, 1 \mathrm{H}), 2.63(\mathrm{dd}, J=18.8,2.6 \mathrm{~Hz}, 1 \mathrm{H})$, $2.23(\mathrm{~d}, J=18.8 \mathrm{~Hz}, 1 \mathrm{H}$ ), 2.10 (ddd, $J=12.4,10.7,7.8 \mathrm{~Hz}, 1 \mathrm{H}$ ), 1.85 (dddd, $J=12.5,6.3$, 2.5, $1.2 \mathrm{~Hz}, 1 \mathrm{H}), 1.52(\mathrm{dd}, J=8.1,4.1 \mathrm{~Hz}, 1 \mathrm{H}), 1.41(\mathrm{p}, J=6.8 \mathrm{~Hz}, 1 \mathrm{H}), 1.00(\mathrm{~d}, J=6.8 \mathrm{~Hz}$, $3 \mathrm{H}$ ), 0.95 (d, $J=6.9 \mathrm{~Hz}, 3 \mathrm{H}$ ), 0.77 (ddd, $J=8.3,5.9,2.5 \mathrm{~Hz}, 1 \mathrm{H}$ ), 0.07 (dd, $J=5.8,4.0 \mathrm{~Hz}$, $1 \mathrm{H})$.

${ }^{13} \mathrm{C}$ NMR $\left(\mathrm{CDCl}_{3}, 101 \mathrm{MHz}\right): \delta=217.5,63.0,41.7,41.2,37.1,32.8,30.7,28.3,28.2,20.0$, 20.0, 17.2.

IR (Diamond-ATR, $\left.\mathrm{CDCl}_{3}\right): \tilde{v}_{\max }=2955(\mathrm{~m}), 2871(w), 1737(\mathrm{vs}), 1464(\mathrm{~m}), 1215(\mathrm{~m})$, $1174(m), 1059(w), 1041(w), 849(w), 614(w)$.

HRMS (ESI) calc. for $\mathrm{C}_{12} \mathrm{H}_{18} \mathrm{NaOS}[\mathrm{M}+\mathrm{Na}]^{+}:$233.0972; found: 233.0968 .

MP $=45-47^{\circ} \mathrm{C}$.

\section{Tetrahydrothiophene 8q}

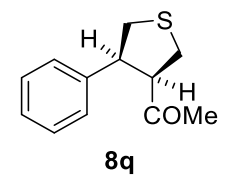

Following the General Procedures GP1 (14 kbar) and GP2, (Z)-benzalacetone (S-23, 73 mg, $0.50 \mathrm{mmol}$ ) was converted into tetrahydrothiophene $\mathbf{8 q}$. Purification by flash-column chromatography on silica gel $(10 \%$ to $20 \%$ diethyl ether in pentane) furnished $\mathbf{8 q}$ (GP1: 73 mg, 71\%; GP2: 34 mg, 33\%) as a colorless oil.

$\operatorname{TLC}\left(15 \%\right.$ diethyl ether in pentane): $R_{\mathrm{f}}=0.17\left(\mathrm{UV}, \mathrm{KMnO}_{4}\right)$

${ }^{1} \mathrm{H}$ NMR $\left(\mathrm{CDCl}_{3}, 400 \mathrm{MHz}\right): \delta=7.32-7.24(\mathrm{~m}, 4 \mathrm{H}), 7.24-7.20(\mathrm{~m}, 1 \mathrm{H}), 3.88(\mathrm{td}, J=6.5$, $5.1 \mathrm{~Hz}, 1 \mathrm{H}), 3.46(\mathrm{dt}, J=8.1,6.8 \mathrm{~Hz}, 1 \mathrm{H}), 3.32(\mathrm{dd}, J=10.8,6.6 \mathrm{~Hz}, 1 \mathrm{H}), 3.22(\mathrm{dd}, J=11.2$, $8.1 \mathrm{~Hz}, 1 \mathrm{H}), 3.16(\mathrm{dd}, J=10.8,5.1 \mathrm{~Hz}, 1 \mathrm{H}), 3.03(\mathrm{dd}, J=11.2,7.1 \mathrm{~Hz}, 1 \mathrm{H}), 1.84(\mathrm{~s}, 3 \mathrm{H})$.

${ }^{13} \mathrm{C} \mathrm{NMR}\left(\mathrm{CDCl}_{3}, 101 \mathrm{MHz}\right): \delta=207.4,139.6,128.8,127.7,127.6,60.7,51.3,36.6,31.3$, 30.8 .

IR (Diamond-ATR, $\left.\mathrm{CDCl}_{3}\right): \tilde{v}_{\max }=2936(w), 1706(v s), 1495(w), 1455(w), 1357(m)$, $1204(w), 1169(m), 753(m), 701(s), 567(m)$.

HRMS (ESI) calc. for $\mathrm{C}_{12} \mathrm{H}_{14} \mathrm{NaOS}[\mathrm{M}+\mathrm{Na}]^{+}:$229.0658; found: 229.0654 . 
Note: Isomerization of the double bond was observed under thermal conditions (GP2), which led to the isolation of (E)-benzalacetone $(\mathbf{S}-22,27 \mathrm{mg}, 37 \%)$ but only traces of its corresponding cycloaddition product $8 \mathrm{r}$. Isomerization did not occur upon heating to $80{ }^{\circ} \mathrm{C}$ in $N, N$ '-dimethylpropylenurea without adding sulfoxide 1a or under high-pressure (14 kbar) conditions.

\section{Tetrahydrothiophene $8 r$}

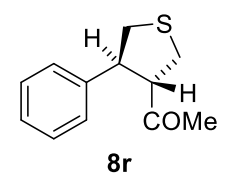

Following the General Procedures GP1 and GP2, (E)-benzalacetone (S-22, $73 \mathbf{m g}$, $0.50 \mathrm{mmol}$ ) was converted into tetrahydrothiophene $8 \mathrm{r}$. Purification by flash-column chromatography on silica gel (10\% ethyl acetate in hexanes) furnished 8r (GP1: $96 \mathrm{mg}, 93 \%$; GP2: $84 \mathrm{mg}, 81 \%$ ) as a pale-yellow solid.

TLC (20\% ethyl acetate in hexanes): $R_{\mathrm{f}}=0.32\left(\mathrm{UV}, \mathrm{KMnO}_{4}\right)$.

${ }^{1} \mathbf{H}$ NMR $\left(\mathrm{CDCl}_{3}, 400 \mathrm{MHz}\right): \delta=7.42-7.16(\mathrm{~m}, 5 \mathrm{H}), 3.62-3.49(\mathrm{~m}, 1 \mathrm{H}), 3.42-3.30(\mathrm{~m}$, $1 \mathrm{H}), 3.23(\mathrm{dd}, J=10.8,7.1 \mathrm{~Hz}, 1 \mathrm{H}), 3.15(\mathrm{dd}, J=8.4,0.7 \mathrm{~Hz}, 2 \mathrm{H}), 3.07(\mathrm{dd}, J=10.8$, $10.0 \mathrm{~Hz}, 1 \mathrm{H}), 1.96(\mathrm{~s}, 3 \mathrm{H})$.

${ }^{13} \mathrm{C}$ NMR $\left(\mathrm{CDCl}_{3}, 101 \mathrm{MHz}\right): \delta=207.9,140.4,129.1,127.5,127.4,62.3,53.1,38.4,33.7$, 30.3.

IR (Diamond-ATR, $\left.\mathrm{CDCl}_{3}\right)$ : $\tilde{v}_{\max }=3027(w), 2934(w), 1708(v s), 1493(m), 1454(m)$, $1356(s), 1169(m), 764(m), 727(m), 699(v s)$.

HRMS (EI) calc. for $\mathrm{C}_{12} \mathrm{H}_{14} \mathrm{OS}[\mathrm{M}]^{+}:$: 206.0760; found: 206.0756 .

MP $=36-38^{\circ} \mathrm{C}$ 


\section{Tetrahydrothiophene 8s}

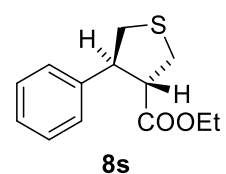

Following the General Procedures GP1 and GP2, (E)-ethyl cinnamate ( $88 \mathrm{mg}, 0.50 \mathrm{mmol}$ ) was converted into tetrahydrothiophene $8 \mathbf{s}$. Purification by flash-column chromatography on silica gel (15\% diethyl ether in pentane) furnished 8s (GP1: 112 mg, 95\%; GP2: 82 mg, 70\%) as a colorless oil.

TLC (20\% diethyl ether in pentane): $R_{\mathrm{f}}=0.50\left(\mathrm{UV}, \mathrm{KMnO}_{4}\right)$

${ }^{1} \mathbf{H}$ NMR $\left(\mathrm{CDCl}_{3}, 599 \mathrm{MHz}\right): \delta=7.36-7.19(\mathrm{~m}, 5 \mathrm{H}), 4.07-3.94(\mathrm{~m}, 2 \mathrm{H}), 3.68-3.59(\mathrm{~m}$, $1 \mathrm{H}), 3.29-3.12(\mathrm{~m}, 4 \mathrm{H}), 3.04(\mathrm{dd}, J=10.8,10.1 \mathrm{~Hz}, 1 \mathrm{H}), 1.06(\mathrm{t}, J=7.1 \mathrm{~Hz}, 3 \mathrm{H})$.

${ }^{13} \mathrm{C}$ NMR $\left(\mathrm{CDCl}_{3}, 151 \mathrm{MHz}\right): \delta=172.6,140.0,128.8,127.4,127.4,60.9,54.9,53.3,37.9$, 34.0, 14.1 .

IR (Diamond-ATR, $\mathrm{CDCl}_{3}$ ): $\tilde{v}_{\max }=2979(w), 2937(w), 1729$ (vs), $1372(w), 1260(m)$, $1177(s), 1157(s), 1031(w), 766(w), 699(s)$.

HRMS (El) calc. for $\mathrm{C}_{13} \mathrm{H}_{16} \mathrm{O}_{2} \mathrm{~S}$ [M] : 236.0866 ; found: 236.0862 .

\section{Tetrahydrothiophene 8t}

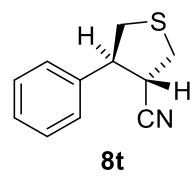

Following the General Procedures GP1 and GP2, $(E)$-cinnamonitrile (65 mg, $0.50 \mathrm{mmol}$ ) was converted into tetrahydrothiophene $\mathbf{8 t}$. Purification by flash-column chromatography on silica gel (10\% diethyl ether in pentane) furnished 8t (GP1: $80 \mathrm{mg}, 84 \%$; GP2: $67 \mathrm{mg}, 70 \%$ ) as a colorless solid. Recrystallization from dichloromethane gave crystals suitable for $\mathrm{X}$-ray analysis.

TLC (10\% diethyl ether in pentane): $R_{\mathrm{f}}=0.30\left(\mathrm{UV}, \mathrm{KMnO}_{4}\right)$

${ }^{1} \mathrm{H}$ NMR $\left(\mathrm{CDCl}_{3}, 599 \mathrm{MHz}\right): \delta=7.41-7.37(\mathrm{~m}, 2 \mathrm{H}), 7.35-7.31(\mathrm{~m}, 3 \mathrm{H}), 3.58(\mathrm{td}, J=9.6$, $7.2 \mathrm{~Hz}, 1 \mathrm{H}), 3.32-3.27(\mathrm{~m}, 2 \mathrm{H}), 3.25-3.21(\mathrm{~m}, 1 \mathrm{H}), 3.21-3.16(\mathrm{~m}, 1 \mathrm{H}), 3.10(\mathrm{dd}, J=11.1$, $9.5 \mathrm{~Hz}, 1 \mathrm{H})$. 
${ }^{13} \mathrm{C}$ NMR $\left(\mathrm{CDCl}_{3}, 151 \mathrm{MHz}\right): \delta=137.7,129.3,128.4,127.2,119.3,53.7,40.2,36.6,33.7$.

IR (Diamond-ATR, $\left.\mathrm{CDCl}_{3}\right): \tilde{v}_{\max }=2940(w), 2241(w), 1602(w), 1498(m), 1456(m)$, $1206(w), 1031(w), 763(s), 726(m), 699(v s)$.

HRMS (El) calc. for $\mathrm{C}_{11} \mathrm{H}_{11} \mathrm{NS}[\mathrm{M}]^{+}:$189.0607; found: 189.0605.

$M P=84-86^{\circ} \mathrm{C}$.

\section{Tetrahydrothiophene $8 \mathrm{u}$}

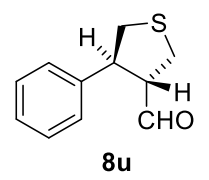

Following the General Procedures GP1 and GP2, (E)-cinnamaldehyde (66 mg, $0.50 \mathrm{mmol}$ ) was converted into tetrahydrothiophene $8 \mathrm{u}$. Purification by flash-column chromatography on silica gel (dichloromethane) furnished 8u (GP1: $68 \mathrm{mg}, 71 \%$; GP2: $58 \mathrm{mg}, 61 \%$ ) as a colorless, amorphous solid.

TLC (20\% ethyl acetate in hexanes): $R_{\mathrm{f}}=0.32\left(\mathrm{UV}, \mathrm{KMnO}_{4}\right)$.

${ }^{1} \mathrm{H}$ NMR $\left(\mathrm{CDCl}_{3}, 400 \mathrm{MHz}\right): \delta=9.59(\mathrm{~d}, J=1.5 \mathrm{~Hz}, 1 \mathrm{H}), 7.41-7.20(\mathrm{~m}, 5 \mathrm{H}), 3.79-3.69(\mathrm{~m}$, $1 \mathrm{H}), 3.26-3.16(\mathrm{~m}, 3 \mathrm{H}), 3.15-3.03(\mathrm{~m}, 2 \mathrm{H})$.

${ }^{13} \mathrm{C}$ NMR $\left(\mathrm{CDCl}_{3}, 101 \mathrm{MHz}\right): \delta=200.7,140.3,129.1,127.6,127.3,61.4,50.5,37.9,30.6$.

IR (Diamond-ATR, $\left.\mathrm{CDCl}_{3}\right): \tilde{v}_{\max }=2934(w), 2721(w), 1722(v s), 1601(w), 1494(w)$, $1455(w), 1248(w), 1070(w), 765(w), 699(s)$.

HRMS (EI) calc. for $\mathrm{C}_{11} \mathrm{H}_{12} \mathrm{OS}$ [M] : 192.0603; found: 192.0602. 


\section{Tetrahydrothiophene $8 \mathrm{v}$}

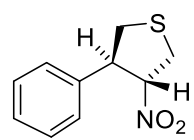

$8 v$

Following the General Procedures GP1 and GP2, $(E)-\beta$-nitrostyrene (75 mg, $0.50 \mathrm{mmol}$ ) was converted into tetrahydrothiophene $\mathbf{8 v}$. Purification by flash-column chromatography on silica gel (10\% diethyl ether in pentane) furnished 8v (GP1: $46 \mathrm{mg}, 44 \%$; GP2: $66 \mathrm{mg}, 63 \%$ ) as a colorless oil.

TLC (10\% diethyl ether in pentane): $R_{\mathrm{f}}=0.30\left(\mathrm{UV}, \mathrm{KMnO}_{4}\right)$

${ }^{1} \mathbf{H}$ NMR $\left(\mathrm{CDCl}_{3}, 599 \mathrm{MHz}\right): \delta=7.38-7.35(\mathrm{~m}, 2 \mathrm{H}), 7.33-7.29(\mathrm{~m}, 3 \mathrm{H}), 5.12-5.07(\mathrm{~m}$, $1 \mathrm{H}), 4.11(\mathrm{q}, J=8.1 \mathrm{~Hz}, 1 \mathrm{H}), 3.58-3.53(\mathrm{~m}, 1 \mathrm{H}), 3.40(\mathrm{dd}, J=11.6,6.6 \mathrm{~Hz}, 1 \mathrm{H}), 3.34(\mathrm{dd}, J$ $=11.3,7.6 \mathrm{~Hz}, 1 \mathrm{H}), 3.13(\mathrm{dd}, J=11.2,8.4 \mathrm{~Hz}, 1 \mathrm{H})$.

${ }^{13} \mathrm{C} \mathrm{NMR}\left(\mathrm{CDCl}_{3}, 151 \mathrm{MHz}\right): \delta=137.4,129.3,128.4,127.3,92.7,53.2,34.9,34.0$.

IR (Diamond-ATR, $\left.\mathrm{CDCl}_{3}\right): \tilde{v}_{\max }=2943(w), 1548(v s), 1495(m), 1456(\mathrm{~m}), 1369(\mathrm{~s})$, $1304(w), 853(w), 766(m), 731(m), 699(s)$.

HRMS (El) calc. for $\mathrm{C}_{10} \mathrm{H}_{11} \mathrm{NO}_{2} \mathrm{~S}$ [M] : 209.0505; found: 209.0504 .

\section{Tetrahydrothiophene $8 w$}

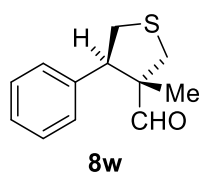

Following the General Procedures GP1 and GP2, a-methyl-(E)-cinnamaldehyde (73 mg, $0.50 \mathrm{mmol}$ ) was converted into tetrahydrothiophene $\mathbf{8 w}$. Purification by flash-column chromatography on silica gel (2\% ethyl acetate in hexanes) furnished 8w (GP1: 68 mg, 66\%; GP2: $27 \mathrm{mg}, 26 \%$ ) as a colorless solid.

$\operatorname{TLC}\left(2 \%\right.$ ethyl acetate in hexanes): $R_{\mathrm{f}}=0.21\left(\mathrm{UV}, \mathrm{KMnO}_{4}\right)$.

${ }^{1} \mathrm{H}$ NMR $\left(\mathrm{CDCl}_{3}, 400 \mathrm{MHz}\right): \delta=9.56(\mathrm{~s}, 1 \mathrm{H}), 7.35-7.30(\mathrm{~m}, 2 \mathrm{H}), 7.29-7.24(\mathrm{~m}, 3 \mathrm{H}), 3.77$ (dd, $J=7.1,5.4 \mathrm{~Hz}, 1 \mathrm{H}), 3.27-3.14(\mathrm{~m}, 3 \mathrm{H}), 2.73(\mathrm{~d}, J=11.2 \mathrm{~Hz}, 1 \mathrm{H}), 0.94(\mathrm{~s}, 3 \mathrm{H})$.

${ }^{13} \mathrm{C}$ NMR $\left(\mathrm{CDCl}_{3}, 101 \mathrm{MHz}\right): \delta=203.0,139.3,128.7,128.5,127.5,61.0,52.1,37.3,35.6$, 16.1. 
IR (Diamond-ATR, $\left.\mathrm{CDCl}_{3}\right): \tilde{v}_{\max }=2929(w), 1724(v s), 1493(m), 1456(s), 1212(w), 932(w)$, $874(w), 774(w), 755(w), 703(s)$.

HRMS (El) calc. for $\mathrm{C}_{12} \mathrm{H}_{14} \mathrm{OS}$ [M] $]^{+}$206.0759; found: 206.0760.

MP $=34-36^{\circ} \mathrm{C}$.

\section{Tetrahydrothiophene $8 x$}

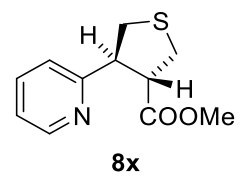

Following the General Procedures GP1 and GP2, pyridine S-26 (82 mg, $0.50 \mathrm{mmol}$ ) was converted into tetrahydrothiophene $\mathbf{8 x}$. Purification by flash-column chromatography on silica gel (25\% ethyl acetate in hexanes) furnished 8x (GP1: $104 \mathrm{mg}, 93 \%$; GP2: $76 \mathrm{mg}, 69 \%$ ) as a colorless, amorphous solid.

$\operatorname{TLC}\left(25 \%\right.$ ethyl acetate in cyclohexane): $R_{\mathrm{f}}=0.31\left(\mathrm{UV}, \mathrm{KMnO}_{4}\right)$.

${ }^{1} \mathrm{H}$ NMR $\left(\mathrm{CDCl}_{3}, 400 \mathrm{MHz}\right): \delta=8.58-8.52(\mathrm{~m}, 1 \mathrm{H}), 7.61(\mathrm{td}, J=7.7,1.9 \mathrm{~Hz}, 1 \mathrm{H}), 7.25-$ $7.21(\mathrm{~m}, 1 \mathrm{H}$ ), 7.15 (ddd, $J=7.6,4.9,1.2 \mathrm{~Hz}, 1 \mathrm{H}$ ), 3.82 (dt, $J=9.8,8.4 \mathrm{~Hz}, 1 \mathrm{H}$ ), $3.64-3.55$ $(\mathrm{m}, 4 \mathrm{H}), 3.28-3.16(\mathrm{~m}, 4 \mathrm{H})$.

${ }^{13} \mathrm{C} \mathrm{NMR}\left(\mathrm{CDCl}_{3}, 101 \mathrm{MHz}\right): \delta=173.4,159.5,149.7,136.7,123.1,122.3,54.1,53.2,52.1$, 36.8, 33.9.

IR (Diamond-ATR, $\left.\mathrm{CDCl}_{3}\right): \tilde{v}_{\max }=2950(w), 1733(v s), 1590(s), 1472(m), 1436(s)$, $1265(m), 1173(s), 1012(w), 789(w), 750(w)$.

HRMS (El) calc. for $\mathrm{C}_{11} \mathrm{H}_{13} \mathrm{NOS}[\mathrm{M}]^{+}:$:223.0662; found: 223.0664 . 


\section{Tetrahydrothiophene $8 \mathrm{y}$}

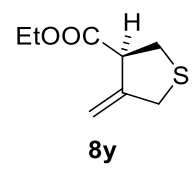

Following the General Procedures GP1 and GP2, ethyl 2,3-butadienoate (56 mg, $0.50 \mathrm{mmol}$ ) was converted into tetrahydrothiophene $\mathbf{8 y}$. Purification by flash-column chromatography on silica gel (20\% dichloromethane in hexanes) furnished 8y (GP1: 42 mg, 48\%; GP2: 38 mg, $44 \%)$ as a colorless oil.

$\operatorname{TLC}(20 \%$ dichloromethane in hexanes $): R_{\mathrm{f}}=0.16\left(\mathrm{KMnO}_{4}\right)$.

${ }^{1} \mathrm{H}$ NMR $\left(\mathrm{CDCl}_{3}, 599 \mathrm{MHz}\right): \delta=5.14-5.09(\mathrm{~m}, 2 \mathrm{H}), 4.26-4.15(\mathrm{~m}, 2 \mathrm{H}), 3.65-3.57(\mathrm{~m}$, 2H), 3.46 (dtt, $J=13.7,1.5,0.7 \mathrm{~Hz}, 1 \mathrm{H}$ ), 3.23 (ddd, $J=11.2,6.4,0.9 \mathrm{~Hz}, 1 \mathrm{H}$ ), 3.05 (ddd, $J=$ $11.3,7.3,0.8 \mathrm{~Hz}, 1 \mathrm{H}), 1.28(\mathrm{td}, J=7.2,0.9 \mathrm{~Hz}, 3 \mathrm{H})$.

${ }^{13} \mathrm{C}$ NMR $\left(\mathrm{CDCl}_{3}, 151 \mathrm{MHz}\right): \delta=171.9,146.9,110.3,61.3,52.4,36.0,33.4,14.3$.

IR (Diamond-ATR, $\left.\mathrm{CDCl}_{3}\right): \tilde{v}_{\max }=1729(v s), 1652(w), 1445(w), 1368(m), 1324(m)$, $1249(m), 1173(s), 1161(s), 1032(m), 902(m)$.

HRMS (EI) calc. for $\mathrm{C}_{8} \mathrm{H}_{12} \mathrm{O}_{2} \mathrm{~S}$ [M] $]^{+}$: 172.0553; found: 172.0553 .

\section{Tetrahydrothiophene (-)-8z}

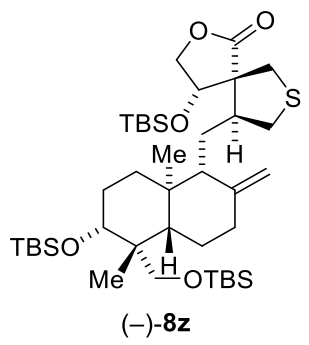

Following the General Procedures GP1 (14 kbar) and GP2, lactone S-28 (173 mg, $0.250 \mathrm{mmol}$, 1 equiv) was converted into tetrahydrothiophene (-)-8z by using sulfoxide $\mathbf{1 a}$ (111 mg, $0.500 \mathrm{mmol}, 2.00$ equiv). Purification by flash-column chromatography on silica gel (10\% diethyl ether in pentane) furnished (-)-8z (GP1: $115 \mathrm{mg}, 61 \%$; GP2: $44 \mathrm{mg}, 23 \%$ ) as a colorless solid. Recrystallization from a mixture of methanol and dichloromethane (1:1) gave crystals suitable for X-ray analysis.

TLC (10\% diethyl ether in pentane): $R_{\mathrm{f}}=0.29\left(\mathrm{UV}, \mathrm{KMnO}_{4}, \mathrm{CAM}\right)$. 
${ }^{1} \mathrm{H}$ NMR $\left(\mathrm{CDCl}_{3}, 599 \mathrm{MHz}\right): \delta=4.85(\mathrm{~d}, J=1.3 \mathrm{~Hz}, 1 \mathrm{H}), 4.59(\mathrm{t}, J=6.9 \mathrm{~Hz}, 1 \mathrm{H}), 4.52(\mathrm{~s}, 1 \mathrm{H})$, 4.37 (dd, $J=9.2,7.1 \mathrm{~Hz}, 1 \mathrm{H}$ ), $3.96(\mathrm{dd}, J=9.2,6.7 \mathrm{~Hz}, 1 \mathrm{H}), 3.88(\mathrm{~d}, J=10.5 \mathrm{~Hz}, 1 \mathrm{H}$ ), 3.57 (dd, $J=10.5,0.9 \mathrm{~Hz}, 1 \mathrm{H}$ ), 3.30 (d, $J=11.0 \mathrm{~Hz}, 1 \mathrm{H}$ ), 3.27 (dd, $J=11.8,4.3 \mathrm{~Hz}, 1 \mathrm{H}$ ), 3.03 (dd, $J=9.4,7.4 \mathrm{~Hz}, 1 \mathrm{H}), 2.83(\mathrm{dd}, J=10.8,9.3 \mathrm{~Hz}, 1 \mathrm{H}), 2.74-2.68(\mathrm{~m}, 1 \mathrm{H}), 2.65(\mathrm{~d}, J=$ $11.0 \mathrm{~Hz}, 1 \mathrm{H}), 2.37-2.33(\mathrm{~m}, 1 \mathrm{H}), 2.15-2.10(\mathrm{~m}, 1 \mathrm{H}), 1.83-1.71(\mathrm{~m}, 4 \mathrm{H}), 1.69-1.63(\mathrm{~m}$, 2H), $1.59(\mathrm{~d}, J=11.5 \mathrm{~Hz}, 1 \mathrm{H}), 1.50-1.46(\mathrm{~m}, 1 \mathrm{H}), 1.13-1.03(\mathrm{~m}, 2 \mathrm{H}), 0.98(\mathrm{~s}, 3 \mathrm{H}), 0.93(\mathrm{~s}$, $9 \mathrm{H}), 0.88(\mathrm{~s}, 9 \mathrm{H}), 0.86(\mathrm{~s}, 9 \mathrm{H}), 0.75(\mathrm{~s}, 3 \mathrm{H}), 0.19(\mathrm{~s}, 3 \mathrm{H}), 0.13(\mathrm{~s}, 3 \mathrm{H}), 0.04(\mathrm{~s}, 3 \mathrm{H}), 0.03(\mathrm{~s}$, $3 \mathrm{H}),-0.02(\mathrm{~s}, 3 \mathrm{H}),-0.02(\mathrm{~s}, 3 \mathrm{H})$.

${ }^{13} \mathrm{C} \mathrm{NMR}\left(\mathrm{CDCl}_{3}, 151 \mathrm{MHz}\right): \delta=177.6,146.8,107.5,79.5,77.6,72.2,64.3,57.9,55.9,55.2$, 49.3 , 44.0, 41.3, 39.5, 39.4, 38.4, 34.6, 28.4, 26.6, 26.1, 26.0, 25.8, 23.7, 23.1, 18.4, 18.2, $18.0,14.5,-3.8,-4.6,-4.7,-4.8,-5.4,-5.6$.

IR (Diamond-ATR, $\left.\mathrm{CDCl}_{3}\right): \tilde{v}_{\max }=2930(m), 1769(m), 1472(w), 1254(m), 1188(w)$, $1093(s), 1044(m), 835(v s), 774(s), 735(m)$.

HRMS (ESI) calc. for $\mathrm{C}_{40} \mathrm{H}_{76} \mathrm{NaO}_{5} \mathrm{SSi}_{3}[\mathrm{M}+\mathrm{Na}]^{+}:$775.4613; found: 775.4601 .

$\left[\alpha_{D}^{20}\right]=-47.8\left(c=2.69, \mathrm{CH}_{2} \mathrm{Cl}_{2}\right)$

MP $=184-186^{\circ} \mathrm{C}$. 


\subsubsection{Styrenes}

\section{Tetrahydrothiophene 9a}

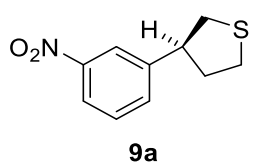

Following the General Procedures GP1 (14 kbar) and GP2, 3-nitrostyrene (75 mg, $0.50 \mathrm{mmol}$ ) was converted into tetrahydrothiophene 9a. Purification by flash-column chromatography on silica gel (2\% diethyl ether in pentane) furnished 9a (GP1: $85 \mathrm{mg}, 81 \%$; GP2: $46 \mathrm{mg}, 44 \%$ ) as a pale-yellow solid.

TLC (2\% diethyl ether in pentane): $R_{\mathrm{f}}=0.23\left(\mathrm{UV}, \mathrm{KMnO}_{4}\right)$.

${ }^{1} \mathrm{H}$ NMR $\left(\mathrm{CDCl}_{3}, 400 \mathrm{MHz}\right): \delta=8.16(\mathrm{~s}, 1 \mathrm{H}), 8.09(\mathrm{dtd}, J=8.2,2.4,1.2 \mathrm{~Hz}, 1 \mathrm{H}), 7.64(\mathrm{dt}, J=$ 8.0, 1.3 Hz, 1H), 7.49 (td, $J=7.9,1.5 \mathrm{~Hz}, 1 \mathrm{H}$ ), 3.46 (ddt, $J=16.0,9.9,6.0 \mathrm{~Hz}, 1 \mathrm{H}$ ), 3.21 (ddd, $J=10.0,6.7,2.4 \mathrm{~Hz}, 1 \mathrm{H}$ ), $3.05-2.96$ (m, 2H), 2.93 (ddd, $J=10.3,8.7,2.6 \mathrm{~Hz}, 1 \mathrm{H}$ ), $2.50-2.41(\mathrm{~m}, 1 \mathrm{H}), 2.16-2.04(\mathrm{~m}, 1 \mathrm{H})$.

${ }^{13} \mathrm{C}$ NMR $\left(\mathrm{CDCl}_{3}, 101 \mathrm{MHz}\right): \delta=148.6,144.4,133.4,129.6,122.1,122.0,49.1,38.0,37.6$, 30.8 .

IR (Diamond-ATR, $\mathrm{CDCl}_{3}$ ): $\tilde{v}_{\max }=2931(m), 2859(w), 1523$ (vs), $1346(v s), 1213(w)$, $1100(w), 928(w), 806(s), 730(s), 683(s)$.

HRMS (ESI) calc. for $\mathrm{C}_{10} \mathrm{H}_{11}{ }^{107} \mathrm{AgNO}_{2} \mathrm{~S}\left[\mathrm{M}+{ }^{107} \mathrm{Ag}\right]^{+}: 315.9556$; found: 315.9542 .

MP $=43-45^{\circ} \mathrm{C}$

\section{Tetrahydrothiophene 9b}

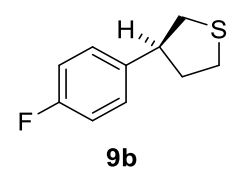

Following the General Procedures GP1 (14 kbar) and GP2, 4-fluorostyrene $(61 \mathrm{mg}$, $0.50 \mathrm{mmol}$ ) was converted into tetrahydrothiophene $9 \mathrm{~b}$. Purification by flash-column chromatography on silica gel (2\% diethyl ether in pentane) furnished 9b (GP1: $48 \mathrm{mg}, 53 \%$; GP2: $14 \mathrm{mg}, 16 \%$ ) as a colorless oil.

TLC (2\% diethyl ether in pentane): $R_{f}=0.22\left(\mathrm{UV}, \mathrm{KMnO}_{4}\right)$. 
${ }^{1} \mathrm{H}$ NMR $\left(\mathrm{CDCl}_{3}, 400 \mathrm{MHz}\right): \delta=7.30-7.23(\mathrm{~m}, 2 \mathrm{H}), 7.06-6.96(\mathrm{~m}, 2 \mathrm{H}), 3.38-3.28(\mathrm{~m}$, $1 \mathrm{H}$ ), 3.16 (ddd, $J=10.5,6.8,0.8 \mathrm{~Hz}, 1 \mathrm{H}$ ), $3.02-2.95(\mathrm{~m}, 2 \mathrm{H}), 2.88(\mathrm{dd}, J=10.4,9.5 \mathrm{~Hz}$, $1 \mathrm{H}), 2.45-2.35(\mathrm{~m}, 1 \mathrm{H}), 2.09-1.95(\mathrm{~m}, 1 \mathrm{H})$.

${ }^{13} \mathrm{C} \mathrm{NMR}\left(\mathrm{CDCl}_{3}, 101 \mathrm{MHz}\right): \delta=161.8(\mathrm{~d}, J=244.9 \mathrm{~Hz}), 137.8(\mathrm{~d}, J=3.2 \mathrm{~Hz}), 128.6(\mathrm{~d}, J=$ $7.8 \mathrm{~Hz}), 115.5$ (d, $J=21.3 \mathrm{~Hz}), 49.07,38.2,37.9,30.9$.

${ }^{19} \mathrm{~F} \mathrm{NMR}\left(\mathrm{CDCl}_{3}, 376 \mathrm{MHz}\right): \delta=-116.26$.

IR (Diamond-ATR, $\left.\mathrm{CDCl}_{3}\right): \tilde{v}_{\max }=2931(m), 2860(w), 1604(w), 1510(v s), 1438(w)$, $1224(s), 1160(m), 832(s), 781(w), 530(m)$.

HRMS (ESI) calc. for $\mathrm{C}_{20} \mathrm{H}_{22}{ }^{107} \mathrm{AgF}_{2} \mathrm{~S}_{2}\left[2 \mathrm{M}+{ }^{107} \mathrm{Ag}\right]^{+}: 471.0176$; found: 471.0157 .

\section{Tetrahydrothiophene 9c}

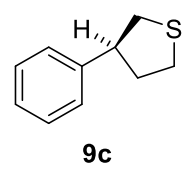

Following the General Procedures GP1 (14 kbar) and GP2, styrene (52 mg, $0.50 \mathrm{mmol}$ ) was converted into tetrahydrothiophene 9c. Purification by flash-column chromatography on silica gel (pentane) furnished 9c (GP1: $36 \mathrm{mg}, 44 \%$; GP2: $18 \mathrm{mg}, 22 \%$ ) as a colorless oil.

TLC (1\% diethyl ether in pentane): $R_{\mathrm{f}}=0.34\left(\mathrm{UV}, \mathrm{KMnO}_{4}\right)$.

${ }^{1} \mathrm{H}$ NMR $\left(\mathrm{CDCl}_{3}, 400 \mathrm{MHz}\right): \delta=7.37-7.22(\mathrm{~m}, 5 \mathrm{H}), 3.41-3.28(\mathrm{~m}, 1 \mathrm{H}), 3.19(\mathrm{ddd}, J=10.4$, 6.8, $0.8 \mathrm{~Hz}, 1 \mathrm{H}), 3.05-2.97(\mathrm{~m}, 2 \mathrm{H}), 2.97-2.89(\mathrm{~m}, 1 \mathrm{H}), 2.48-2.37(\mathrm{~m}, 1 \mathrm{H}), 2.13-2.01$ $(\mathrm{m}, 1 \mathrm{H})$.

${ }^{13} \mathrm{C}$ NMR $\left(\mathrm{CDCl}_{3}, 101 \mathrm{MHz}\right): \delta=142.2,128.7,127.2,126.9,49.9,38.1,37.9,31.1$.

IR (Diamond-ATR, $\left.\mathrm{CDCl}_{3}\right): \tilde{v}_{\max }=2933(s), 1601(w), 1493(m), 1456(m), 1262(w)$, $1210(m), 763(m), 725(m), 698(v s), 522(m)$.

HRMS (ESI) calc. for $\mathrm{C}_{20} \mathrm{H}_{24}{ }^{107} \mathrm{AgS}_{2}\left[2 \mathrm{M}+{ }^{107} \mathrm{Ag}\right]^{+}: 435.0365$; found: 435.0351 . 


\section{Tetrahydrothiophene 9d}

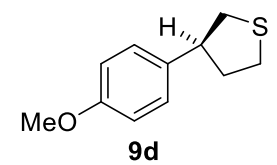

Following the General Procedures GP1 (14 kbar) and GP2, 4-methoxystyrene (67 mg, $0.50 \mathrm{mmol}$ ) was converted into tetrahydrothiophene $9 \mathrm{~d}$. Purification by flash-column chromatography on silica gel (1\% diethyl ether in pentane) furnished 9d (GP1: $55 \mathrm{mg}, 56 \%$; GP2: $4.4 \mathrm{mg}, 5 \%$ ) as a colorless, amorphous solid.

TLC (1\% diethyl ether in pentane): $R_{\mathrm{f}}=0.20\left(\mathrm{UV}, \mathrm{KMnO}_{4}\right)$.

${ }^{1} \mathrm{H}$ NMR $\left(\mathrm{CDCl}_{3}, 400 \mathrm{MHz}\right): \delta=7.24-7.17(\mathrm{~m}, 2 \mathrm{H}), 6.89-6.83(\mathrm{~m}, 2 \mathrm{H}), 3.80(\mathrm{~s}, 3 \mathrm{H}), 3.29$ (dddd, $J=11.0,9.8,6.7,5.2 \mathrm{~Hz}, 1 \mathrm{H}$ ), 3.14 (ddd, $J=10.3,6.8,0.8 \mathrm{~Hz}, 1 \mathrm{H}$ ), $3.02-2.93$ (m, $2 \mathrm{H}), 2.87(\mathrm{dd}, J=10.4,9.7 \mathrm{~Hz}, 1 \mathrm{H}), 2.43-2.33(\mathrm{~m}, 1 \mathrm{H}), 2.08-1.95(\mathrm{~m}, 1 \mathrm{H})$.

${ }^{13} \mathrm{C} \mathrm{NMR}\left(\mathrm{CDCl}_{3}, 101 \mathrm{MHz}\right): \delta=158.5,134.1,128.1,114.1,55.4,49.1,38.2,38.0,31.0$.

IR (Diamond-ATR, $\mathrm{CDCl}_{3}$ ): $\tilde{v}_{\max }=2933(m), 1611(w), 1513(v s), 1462(w), 1304(w)$, $1246(s), 1179(m), 1035(m), 828(m), 537(w)$.

HRMS (ESI) calc. for $\mathrm{C}_{22} \mathrm{H}_{28}{ }^{107} \mathrm{AgO}_{2} \mathrm{~S}_{2}\left[2 \mathrm{M}+{ }^{107} \mathrm{Ag}\right]^{+:}$: 495.0576; found: 495.0562 .

\section{Tetrahydrothiophene $9 \mathrm{e}$}

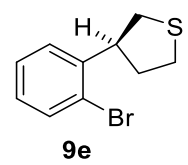

Following the General Procedures GP1 (14 kbar) and GP2, 2-bromostyrene (92 mg, $0.50 \mathrm{mmol}$ ) was converted into tetrahydrothiophene $9 \mathrm{e}$. Purification by flash-column chromatography on silica gel (pentane) furnished 9e (GP1: 75 mg, 62\%; GP2: $47 \mathrm{mg}, 39 \%$ ) as a yellow oil.

TLC (pentane): $R_{\mathrm{f}}=0.19\left(\mathrm{UV}, \mathrm{KMnO}_{4}\right)$.

${ }^{1} \mathrm{H} \operatorname{NMR}\left(\mathrm{CDCl}_{3}, 400 \mathrm{MHz}\right): \delta=7.57(\mathrm{dd}, J=8.0,1.3 \mathrm{~Hz}, 1 \mathrm{H}), 7.41(\mathrm{dd}, J=7.8,1.6 \mathrm{~Hz}, 1 \mathrm{H})$, 7.29 (td, $J=7.6,1.2 \mathrm{~Hz}, 1 \mathrm{H}), 7.09$ (td, $J=7.7,1.7 \mathrm{~Hz}, 1 \mathrm{H}), 3.84-3.74(\mathrm{~m}, 1 \mathrm{H}), 3.26$ (dd, $J=$ 10.5, $6.8 \mathrm{~Hz}, 1 \mathrm{H}), 3.03-2.91(\mathrm{~m}, 2 \mathrm{H}), 2.83(\mathrm{dd}, J=10.5,8.6 \mathrm{~Hz}, 1 \mathrm{H}), 2.43-2.34(\mathrm{~m}, 1 \mathrm{H})$, $2.15-2.03(\mathrm{~m}, 1 \mathrm{H})$. 
${ }^{13} \mathrm{C} \mathrm{NMR}\left(\mathrm{CDCl}_{3}, 101 \mathrm{MHz}\right): \delta=141.2,133.2,128.3,127.9,127.1,124.9,48.3,37.0,36.5$, 30.6.

IR (Diamond-ATR, $\left.\mathrm{CDCl}_{3}\right): \tilde{v}_{\max }=2934(m), 2859(w), 1530(w), 1471(s), 1437(s), 1260(w)$, $1213(w), 1022(s), 754(v s), 739(m)$.

HRMS (ESI) calc. for $\mathrm{C}_{10} \mathrm{H}_{11}{ }^{107} \mathrm{AgBrS}\left[\mathrm{M}+{ }^{107} \mathrm{Ag}\right]^{+}: 348.8810$; found: 348.8795 .

\section{Tetrahydrothiophene $9 f$}

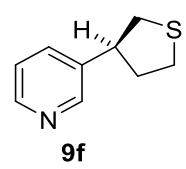

Following the General Procedures GP1 (14 kbar) and GP2, 3-vinylpyridine (S-30, $53 \mathrm{mg}$, $0.50 \mathrm{mmol}$ ) was converted into tetrahydrothiophene 9f. Purification by flash-column chromatography on silica gel (50\% diethyl ether in pentane to diethyl ether) furnished $9 \mathbf{f}$ (GP1: 59 mg, 72\%; GP2: 22 mg, 27\%) as a yellow oil.

TLC (diethyl ether): $R_{\mathrm{f}}=0.21\left(\mathrm{UV}, \mathrm{KMnO}_{4}\right)$.

${ }^{1} \mathrm{H}$ NMR $\left(\mathrm{CDCl}_{3}, 400 \mathrm{MHz}\right): \delta=8.55(\mathrm{~d}, J=2.3 \mathrm{~Hz}, 1 \mathrm{H}), 8.48(\mathrm{dd}, J=4.8,1.6 \mathrm{~Hz}, 1 \mathrm{H}), 7.61$ (dt, $J=7.9,2.0 \mathrm{~Hz}, 1 \mathrm{H}$ ), 7.24 (ddd, $J=7.8,4.8,0.9 \mathrm{~Hz}, 1 \mathrm{H}$ ), $3.40-3.30(\mathrm{~m}, 1 \mathrm{H}), 3.22-3.14$ (m, 1H), $3.02-2.95(\mathrm{~m}, 2 \mathrm{H}), 2.89(\mathrm{dd}, J=10.5,9.2 \mathrm{~Hz}, 1 \mathrm{H}), 2.46-2.37(\mathrm{~m}, 1 \mathrm{H}), 2.05$ (dddd, $J=12.3,10.2,9.1,7.7 \mathrm{~Hz}, 1 \mathrm{H}$ ).

${ }^{13} \mathrm{C} \mathrm{NMR}\left(\mathrm{CDCl}_{3}, 101 \mathrm{MHz}\right): \delta=149.1,148.4,137.6,134.3,123.6,47.1,37.9,37.6,30.9$.

IR (Diamond-ATR, $\left.\mathrm{CDCl}_{3}\right): \tilde{v}_{\max }=2932(m), 2858(w), 1574(w), 1479(m), 1422(s), 1212$ $(w), 1025(m), 809(m), 712(v s), 613(w)$.

HRMS (ESI) calc. for $\mathrm{C}_{9} \mathrm{H}_{12} \mathrm{NS}[\mathrm{M}+\mathrm{H}]^{+}:$166.0685; found: 166.0679 . 


\section{Tetrahydrothiophene $9 \mathrm{~g}$}

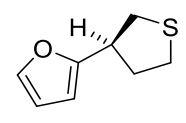

$9 \mathrm{~g}$

Following the General Procedures GP1 (14 kbar) and GP2, 2-vinylfuran (S-32, $47 \mathrm{mg}$, $0.50 \mathrm{mmol}$ ) was converted into tetrahydrothiophene $\mathbf{9 g}$. Purification by flash-column chromatography on silica gel (1\% to $10 \%$ dichloromethane in cyclohexane) furnished $\mathbf{9 g}$ (GP1: $21 \mathrm{mg}, 27 \%$; GP2: $10 \mathrm{mg}, 13 \%$ ) as a colorless oil.

TLC (10\% dichloromethane in cyclohexane): $R_{\mathrm{f}}=0.31(\mathrm{CAM})$.

${ }^{1} \mathrm{H}$ NMR $\left(\mathrm{CDCl}_{3}, 400 \mathrm{MHz}\right): \delta=7.32(\mathrm{dd}, J=1.9,0.8 \mathrm{~Hz}, 1 \mathrm{H}), 6.30(\mathrm{dd}, J=3.2,1.9 \mathrm{~Hz}, 1 \mathrm{H})$, 6.10 (dt, $J=3.2,0.9 \mathrm{~Hz}, 1 \mathrm{H}$ ), 3.43 (dddd, $J=14.9,7.9,5.9,0.9 \mathrm{~Hz}, 1 \mathrm{H}), 3.18-3.11(\mathrm{~m}, 1 \mathrm{H})$, $2.99-2.90$ (m, 3H), 2.36 (dq, $J=12.4,5.4 \mathrm{~Hz}, 1 \mathrm{H}$ ), 2.12 (ddt, $J=12.4,9.5,8.0 \mathrm{~Hz}, 1 \mathrm{H}$ ).

${ }^{13} \mathrm{C}$ NMR $\left(\mathrm{CDCl}_{3}, 101 \mathrm{MHz}\right): \delta=155.8,141.4,110.2,104.8,43.0,35.7,35.5,30.7$.

IR (Diamond-ATR, $\left.\mathrm{CDCl}_{3}\right): \tilde{v}_{\max }=2933(m), 2862(w), 1505(w), 1439(w), 1146(w), 1012$ $(m), 932(w), 804(w), 732(v s), 598(m)$.

HRMS (ESI) calc. for $\mathrm{C}_{16} \mathrm{H}_{20}{ }^{107} \mathrm{AgO}_{2} \mathrm{~S}_{2}\left[2 \mathrm{M}+{ }^{107} \mathrm{Ag}\right]^{+}: 414.9945$; found: 414.9932 .

\section{Tetrahydrothiophene $9 \mathrm{~h}$}

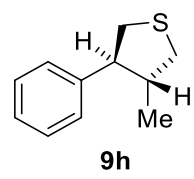

Following the General Procedures GP1 (14 kbar) and GP2, trans- $\beta$-methylstyrene (59 mg, $0.50 \mathrm{mmol}$ ) was converted into tetrahydrothiophene $9 \mathrm{~h}$. Purification by flash-column chromatography on silica gel ( $1 \%$ to $2 \%$ dichloromethane in pentane) furnished $9 \mathrm{~h}$ (GP1: $15 \mathrm{mg}, 17 \%$; GP2: not detected) as a pale-yellow oil.

$\operatorname{TLC}\left(5 \%\right.$ dichloromethane in pentane): $R_{\mathrm{f}}=0.56\left(\mathrm{UV}, \mathrm{KMnO}_{4}\right)$.

${ }^{1} \mathrm{H}$ NMR $\left(\mathrm{CDCl}_{3}, 400 \mathrm{MHz}\right): \delta=7.37-7.30(\mathrm{~m}, 2 \mathrm{H}), 7.24(\mathrm{tt}, J=7.9,1.5 \mathrm{~Hz}, 3 \mathrm{H}), 3.17(\mathrm{dd}, J$ $=10.6,7.0 \mathrm{~Hz}, 1 \mathrm{H}), 3.10(\mathrm{dd}, J=10.4,6.6 \mathrm{~Hz}, 1 \mathrm{H}), 3.02$ (t, $J=10.6 \mathrm{~Hz}, 1 \mathrm{H}), 2.78$ (td, $J=$ 10.7, $7.0 \mathrm{~Hz}, 1 \mathrm{H}$ ), 2.69 (t, $J=10.3 \mathrm{~Hz}, 1 \mathrm{H}$ ), 2.35 (tp, $J=10.5,6.5 \mathrm{~Hz}, 1 \mathrm{H}$ ), 0.95 (d, $J=$ $6.4 \mathrm{~Hz}, 3 \mathrm{H})$. 
${ }^{13} \mathrm{C}$ NMR $\left(\mathrm{CDCl}_{3}, 101 \mathrm{MHz}\right): \delta=141.7,128.8,127.7,127.0,57.0,45.0,38.9,38.6,17.4$.

IR (Diamond-ATR, $\left.\mathrm{CDCl}_{3}\right): \tilde{v}_{\max }=2957(m), 2926(m), 1731(w), 1496(w), 1455(m)$, $1202(w), 845(w), 762(m), 724(m), 699(s)$.

HRMS (ESI) calc. for $\mathrm{C}_{11} \mathrm{H}_{14}{ }^{107} \mathrm{AgS}\left[\mathrm{M}+{ }^{107} \mathrm{Ag}\right]^{+}:$284.9862; found: 284.9839 .

\section{Tetrahydrothiophene 9i}

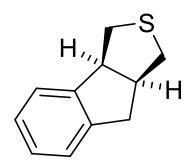

9i

Following the General Procedures GP1 (14 kbar) and GP2, indene (58 mg, $0.50 \mathrm{mmol}$ ) was converted into tetrahydrothiophene $9 \mathbf{i}$. Purification by flash-column chromatography on silica gel (5\% to $50 \%$ dichloromethane in pentane) furnished 9i (GP1: $17 \mathrm{mg}, 19 \%$; GP2: not detected) as a yellow oil.

TLC (2\% diethylether in pentane): $R_{\mathrm{f}}=0.32\left(\mathrm{UV}, \mathrm{KMnO}_{4}\right)$.

${ }^{1} \mathrm{H}$ NMR $\left(\mathrm{CDCl}_{3}, 400 \mathrm{MHz}\right): \delta=7.19(\mathrm{td}, J=4.3,2.5 \mathrm{~Hz}, 4 \mathrm{H}), 3.98(\mathrm{td}, J=7.9,3.6 \mathrm{~Hz}, 1 \mathrm{H})$, $3.34-3.25(\mathrm{~m}, 2 \mathrm{H}), 3.20(\mathrm{dd}, J=16.0,8.1 \mathrm{~Hz}, 1 \mathrm{H}), 3.11-2.95(\mathrm{~m}, 2 \mathrm{H}), 2.84(\mathrm{dd}, J=16.0$, $3.4 \mathrm{~Hz}, 1 \mathrm{H}), 2.61(\mathrm{dd}, J=11.4,6.0 \mathrm{~Hz}, 1 \mathrm{H})$.

${ }^{13} \mathrm{C}$ NMR $\left(\mathrm{CDCl}_{3}, 101 \mathrm{MHz}\right): \delta=145.21,142.69,127.24,127.03,124.84,124.24,54.22$, 48.28, 39.16, 38.57, 38.40.

IR (Diamond-ATR, $\left.\mathrm{CDCl}_{3}\right): \widetilde{v}_{\max }=3019(w), 2928(\mathrm{~s}), 2542(\mathrm{~m}), 1481(\mathrm{~m}), 1457(\mathrm{~m}), 1438$ $(\mathrm{m}), 1359(w), 818(\mathrm{w}), 751(v s), 713(\mathrm{~m})$.

HRMS (ESI) calc. for $\mathrm{C}_{22} \mathrm{H}_{24}{ }^{107} \mathrm{AgS}_{2}\left[2 \mathrm{M}^{107} \mathrm{Ag}\right]^{+}: 459.0359$; found: 459.0348 . 


\subsubsection{1,4- vs. 1,6-Addition and [6+3]-Cycloaddition}

\section{Tetrahydrothiophenes $10 \mathrm{a}$ and $10 \mathrm{~b}$}

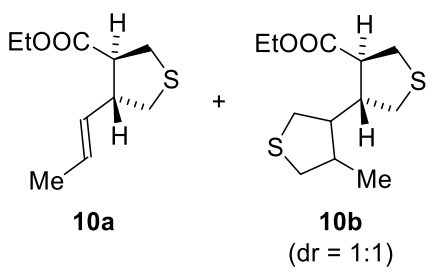

Following the General Procedures GP1 and GP2, ethyl sorbate $(70 \mathrm{mg}, 0.50 \mathrm{mmol}$ ) was converted into tetrahydrothiophenes $10 \mathrm{a}$ and 10b. Purification by flash-column chromatography on silica gel ( $5 \%$ diethyl ether in pentane) furnished monoadduct 10a (GP1: $41 \mathrm{mg}, 41 \%$; GP2: $21 \mathrm{mg}, 21 \%$ ) and an inseparable mixture of diastereomers of double adduct 10b (GP1: $15 \mathrm{mg}, 12 \%, \mathrm{dr}=1: 1$; GP2: not detected) as colorless oils.

\section{Monoadduct 10a:}

TLC (5\% diethyl ether in pentane): $R_{\mathrm{f}}=0.21\left(\mathrm{UV}, \mathrm{KMnO}_{4}\right)$.

${ }^{1} \mathrm{H}$ NMR $\left(\mathrm{CDCl}_{3}, 400 \mathrm{MHz}\right): \delta=5.59(\mathrm{dqd}, J=15.2,6.4,0.8 \mathrm{~Hz}, 1 \mathrm{H}), 5.35(\mathrm{ddq}, J=15.2,7.9$, $1.7 \mathrm{~Hz}, 1 \mathrm{H}), 4.23-4.07(\mathrm{~m}, 2 \mathrm{H}), 3.17-2.95(\mathrm{~m}, 4 \mathrm{H}), 2.78-2.68(\mathrm{~m}, 2 \mathrm{H}), 1.65$ (ddd, $J=6.5$, $1.7,0.5 \mathrm{~Hz}, 3 \mathrm{H}), 1.24(\mathrm{t}, J=7.1 \mathrm{~Hz}, 3 \mathrm{H})$.

${ }^{13} \mathrm{C} \mathrm{NMR}\left(\mathrm{CDCl}_{3}, 101 \mathrm{MHz}\right): \delta=172.9,129.8,127.9,60.9,54.0,51.2,36.3,33.6,18.0,14.4$.

IR (Diamond-ATR, $\mathrm{CDCl}_{3}$ ): $\tilde{v}_{\max }=2937(w), 1730(v s), 1446(w), 1371(m), 1343(w)$, $1252(m), 1182(s), 1158(s), 1037(m), 965(m)$.

HRMS (ESI) calc. for $\mathrm{C}_{10} \mathrm{H}_{16} \mathrm{NaO}_{2} \mathrm{~S}[\mathrm{M}+\mathrm{Na}]^{+}: 223.0763$ found: 223.0761 .

Double adduct $10 \mathrm{~b}(d r=1: 1)$ :

TLC (5\% diethyl ether in pentane): $R_{\mathrm{f}}=0.15\left(\mathrm{KMnO}_{4}\right)$.

${ }^{1} \mathrm{H}$ NMR $\left(\mathrm{CDCl}_{3}, 400 \mathrm{MHz}\right): \delta=4.23-4.12(\mathrm{~m}, 4 \mathrm{H}), 3.15-2.99(\mathrm{~m}, 5 \mathrm{H}), 2.99-2.65(\mathrm{~m}$, $13 \mathrm{H}), 2.56-2.47(\mathrm{~m}, 2 \mathrm{H}), 2.05(\mathrm{tt}, J=7.5,6.5 \mathrm{~Hz}, 1 \mathrm{H}), 1.96-1.82(\mathrm{~m}, 3 \mathrm{H}), 1.28(\mathrm{t}, J=$ $7.1 \mathrm{~Hz}, 6 \mathrm{H}), 1.12-1.06(\mathrm{~m}, 6 \mathrm{H})$.

${ }^{13} \mathrm{C} \mathrm{NMR}\left(\mathrm{CDCl}_{3}, 101 \mathrm{MHz}\right): \delta=173.8,172.9,61.3,61.2,52.4,52.0,50.4,49.8,48.0,47.3$, $42.2,41.1,38.6,38.6,35.5,35.0,34.1,33.6,30.9,30.1,19.0,17.2,14.4,14.3$.

IR (Diamond-ATR, $\mathrm{CDCl}_{3}$ ): $\tilde{v}_{\max }=2959(m), 2931(m), 2869(w), 1730(v s), 1451(w)$, $1373(w), 1254(m), 1156(s), 1096(w), 1033(m)$. 
HRMS (El) calc. for $\mathrm{C}_{12} \mathrm{H}_{20} \mathrm{O}_{2} \mathrm{~S}_{2}[\mathrm{M}]^{+}:$260.0899; found: 260.0892 .

\section{Tetrahydrothiophene 10c}

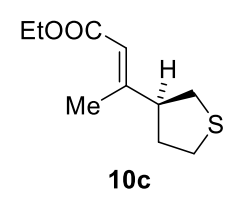

Following the General Procedures GP1 and GP2, enone S-34 (70 mg, $0.50 \mathrm{mmol}$ ) was converted into tetrahydrothiophene $10 \mathrm{c}$. Purification by flash-column chromatography on silica gel (10\% diethyl ether in pentane) furnished 10c (GP1: 89 mg, 89\%; GP2: $63 \mathrm{mg}, 63 \%$ ) as a colorless oil.

TLC (10\% diethyl ether in hexanes): $R_{\mathrm{f}}=0.31\left(\mathrm{UV}, \mathrm{KMnO}_{4}\right)$

${ }^{1} \mathrm{H}$ NMR $\left(\mathrm{CDCl}_{3}, 400 \mathrm{MHz}\right): \delta=5.80(\mathrm{p}, J=1.2 \mathrm{~Hz}, 1 \mathrm{H}), 4.15(\mathrm{q}, J=7.2 \mathrm{~Hz}, 2 \mathrm{H}), 2.98-2.87$ $(\mathrm{m}, 3 \mathrm{H}), 2.83-2.72(\mathrm{~m}, 2 \mathrm{H}), 2.25-2.16(\mathrm{~m}, 4 \mathrm{H}), 1.95-1.83(\mathrm{~m}, 1 \mathrm{H}), 1.28(\mathrm{t}, J=7.1 \mathrm{~Hz}$, $3 \mathrm{H})$.

${ }^{13} \mathrm{C}$ NMR $\left(\mathrm{CDCl}_{3}, 101 \mathrm{MHz}\right): \delta=166.8,158.3,116.0,59.9,53.6,35.1,34.8,30.8,17.4,14.5$.

IR (Diamond-ATR, $\left.\mathrm{CDCl}_{3}\right): \tilde{v}_{\max }=2936(w), 1712(v s), 1643(s), 1441(w), 1368(w), 1222(s)$, $1198(m), 1146(v s), 1042(m), 873(w)$.

HRMS (EI) calc. for $\mathrm{C}_{10} \mathrm{H}_{16} \mathrm{O}_{2} \mathrm{~S}$ [M]+: 200.0866; found: 200.0863. 


\section{Tetrahydro-4H-thiopyran-4-one 11}

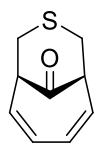

11

Following the General Procedures GP1 and GP2, tropone $(53 \mathrm{mg}, 0.50 \mathrm{mmol}$ ) was converted into tetrahydro-4H-thiopyran-4-one 11. Purification by flash-column chromatography on silica gel (50\% dichloromethane in pentane) furnished 11 (GP1: $44 \mathrm{mg}$, 53\%; GP2: $24 \mathrm{mg}, 28 \%$ ) as a colorless solid. Recrystallization from diethyl ether gave crystals suitable for X-ray analysis.

TLC $\left(60 \%\right.$ dichloromethane in pentane): $R_{\mathrm{f}}=0.22\left(\mathrm{UV}, \mathrm{KMnO}_{4}\right)$.

${ }^{1} \mathbf{H}$ NMR $\left(\mathrm{CDCl}_{3}, 400 \mathrm{MHz}\right): \delta=6.12-6.05(\mathrm{~m}, 2 \mathrm{H}), 5.63-5.52(\mathrm{~m}, 2 \mathrm{H}), 3.60-3.52(\mathrm{~m}$, 2H), 3.33 (ddt, $J=13.8,4.6,1.6 \mathrm{~Hz}, 2 \mathrm{H}), 2.94-2.85(\mathrm{~m}, 2 \mathrm{H})$.

${ }^{13} \mathrm{C}$ NMR $\left(\mathrm{CDCl}_{3}, 101 \mathrm{MHz}\right): \delta=206.3,127.4,126.2,54.2,39.0$.

IR (Diamond-ATR, CDCl $)_{3}$ : $\widetilde{v}_{\max }=2920(w), 1709(s), 1694(s), 1419(w), 1316(m), 1296(m)$, $1103(w), 873(m), 775(m), 706(v s)$.

HRMS (EI) calc. for $\mathrm{C}_{9} \mathrm{H}_{10} \mathrm{OS}[\mathrm{M}]^{+}:$166.0447; found: 166.0444 .

$\mathbf{M P}=117-119^{\circ} \mathrm{C}$. 


\subsubsection{Alkynes}

\section{Dihydrothiophene 12a}

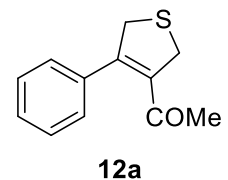

Following the General Procedures GP1 (14 kbar) and GP2, alkyne S-36 (72 mg, $0.50 \mathrm{mmol}$ ) was converted into thiophene 12a. Purification by flash-column chromatography on silica gel ( $30 \%$ to $50 \%$ dichloromethane in pentane) furnished 12a (GP1: $80 \mathrm{mg}, 79 \%$; GP2: $46 \mathrm{mg}$, $45 \%)$ as a yellowish oil.

$\operatorname{TLC}(50 \%$ dichloromethane in pentane $): R_{\mathrm{f}}=0.30\left(\mathrm{UV}, \mathrm{KMnO}_{4}\right)$

${ }^{1} \mathrm{H}$ NMR $\left(\mathrm{CDCl}_{3}, 400 \mathrm{MHz}\right): \delta=7.40(\mathrm{~m}, 3 \mathrm{H}), 7.23(\mathrm{~m}, 2 \mathrm{H}), 4.30-4.09(\mathrm{~m}, 4 \mathrm{H}), 1.82(\mathrm{~s}, 3 \mathrm{H})$.

${ }^{13} \mathrm{C}$ NMR $\left(\mathrm{CDCl}_{3}, 101 \mathrm{MHz}\right): \delta=198.8,150.2,138.4,136.4,129.1,129.0,127.7,46.2,40.9$, 30.0 .

IR (Diamond-ATR, $\left.\mathrm{CDCl}_{3}\right): \tilde{v}_{\max }=2917(w), 1661(v s), 1443(m), 1359(m), 1322(m)$, $1235(w), 1110(w), 772(m), 741(m), 700(s)$.

HRMS (ESI) calc. for $\mathrm{C}_{12} \mathrm{H}_{12} \mathrm{NaO}_{4} \mathrm{~S}[\mathrm{M}+\mathrm{Na}]^{+}:$227.0501; found: 227.0501 .

\section{Dihydrothiophene 12b}

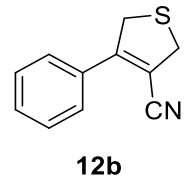

Following the General Procedures GP1 (14 kbar) and GP2, 3-phenylpropiolonitrile (64 mg, $0.50 \mathrm{mmol}$ ) was converted into dihydrothiophene 12b. Purification by flash-column chromatography on silica gel (15\% dichloromethane in pentane) furnished 12b (GP1: $59 \mathrm{mg}$, 63\%; GP2: 32 mg, 34\%) as pale-yellow solid.

TLC (25\% dichloromethane in pentane): $R_{\mathrm{f}}=0.11\left(\mathrm{UV}, \mathrm{KMnO}_{4}\right)$.

${ }^{1} \mathbf{H}$ NMR $\left(\mathrm{CDCl}_{3}, 400 \mathrm{MHz}\right): \delta=7.65-7.59(\mathrm{~m}, 2 \mathrm{H}), 7.48-7.40(\mathrm{~m}, 3 \mathrm{H}), 4.26(\mathrm{dd}, J=5.2$, $4.0 \mathrm{~Hz}, 2 \mathrm{H}), 4.08$ (dd, $J=5.2,4.0 \mathrm{~Hz}, 2 \mathrm{H})$.

${ }^{13} \mathrm{C}$ NMR $\left(\mathrm{CDCl}_{3}, 101 \mathrm{MHz}\right): \delta=156.7,132.6,130.6,129.0,127.4,116.2,106.8,41.6,40.3$. 
IR (Diamond-ATR, $\left.\mathrm{CDCl}_{3}\right): \tilde{v}_{\max }=2911(w), 2214(m), 1621(w), 1492(w), 1442(s)$, $1215(w), 847(m), 766(v s), 691(v s), 605(s)$.

HRMS (ESI) calc. for $\mathrm{C}_{11} \mathrm{H}_{9} \mathrm{NaNS}[\mathrm{M}+\mathrm{Na}]^{+}: 210.0348$; found: 210.0343 .

MP: $59-61^{\circ} \mathrm{C}$.

\section{Dihydrothiophene 12c}

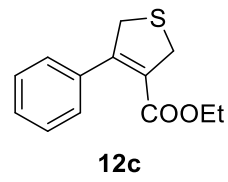

Following the General Procedures GP1 (14 kbar) and GP2, ethyl phenylpropiolate $(87 \mathrm{mg}$, $0.50 \mathrm{mmol}$ ) was converted into dihydrothiophene 12c. Purification by flash-column chromatography on silica gel (30\% dichloromethane in pentane) furnished 12c (GP1: $57 \mathrm{mg}$, 49\%; GP2: $25 \mathrm{mg}, 21 \%$ ) as a yellowish oil.

$\operatorname{TLC}(50 \%$ dichloromethane in pentane $): R_{\mathrm{f}}=0.41\left(\mathrm{UV}, \mathrm{KMnO}_{4}\right)$.

${ }^{1} \mathbf{H}$ NMR $\left(\mathrm{CDCl}_{3}, 400 \mathrm{MHz}\right): \delta=7.38-7.30(\mathrm{~m}, 3 \mathrm{H}), 7.26-7.20(\mathrm{~m}, 2 \mathrm{H}), 4.17(\mathrm{dd}, J=4.8$, $0.8 \mathrm{~Hz}, 4 \mathrm{H}), 4.03$ (q, $J=7.1 \mathrm{~Hz}, 2 \mathrm{H}), 1.04$ (t, $J=7.1 \mathrm{~Hz}, 3 \mathrm{H})$.

${ }^{13} \mathrm{C}$ NMR $\left(\mathrm{CDCl}_{3}, 101 \mathrm{MHz}\right): \delta=164.6,151.5,136.2,128.4,128.4,128.2,127.4,60.6,45.1$, $40.2,13.8$.

IR (Diamond-ATR, $\mathrm{CDCl}_{3}$ ): $\tilde{v}_{\max }=2980(w), 1701(v s), 1443(m), 1326(s), 1200(v s)$, $1120(s), 1044(s), 855(m), 762(s), 696(v s)$.

HRMS (ESI) calc. for $\mathrm{C}_{13} \mathrm{H}_{14} \mathrm{NaO}_{2} \mathrm{~S}[\mathrm{M}+\mathrm{Na}]^{+}: 257.0607$; found: 257.0600 . 


\section{Dihydrothiophene 12d}

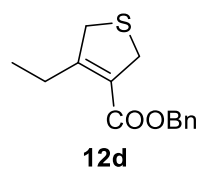

Following the General Procedures GP1 (14 kbar) and GP2, benzyl ester S-38 (101 mg, $0.50 \mathrm{mmol}$ ) was converted into dihydrothiophene 11d. Purification by flash-column chromatography on silica gel (10\% dichloromethane in pentane) furnished 11d (GP1: $60 \mathrm{mg}$, 48\%; GP2: 17 mg, 14\%) as a pale-yellow, amorphous solid.

TLC (30\% dichloromethane in pentane): $\mathrm{R}_{f}=0.15\left(\mathrm{UV}, \mathrm{KMnO}_{4}\right)$.

${ }^{1} \mathrm{H}$ NMR $\left(\mathrm{CDCl}_{3}, 400 \mathrm{MHz}\right) \delta=7.42-7.30(\mathrm{~m}, 5 \mathrm{H}), 5.20(\mathrm{~s}, 2 \mathrm{H}), 4.07-3.96(\mathrm{~m}, 2 \mathrm{H}), 3.94-$ $3.88(\mathrm{~m}, 2 \mathrm{H}), 2.66(\mathrm{q}, J=7.4,8.0 \mathrm{~Hz}, 2 \mathrm{H}), 1.09(\mathrm{t}, J=7.6 \mathrm{~Hz}, 3 \mathrm{H})$.

${ }^{13} \mathrm{C} \mathrm{NMR}\left(\mathrm{CDCl}_{3}, 101 \mathrm{MHz}\right) \delta=164.4,159.0,136.1,128.7,128.3,128.2,125.3,66.2,43.0$, $39.5,23.8,12.6$.

IR (Diamond-ATR, $\left.\mathrm{CDCl}_{3}\right): \tilde{v}_{\max }=2968(w), 2932(m), 1713(s), 1645(m), 1455(w)$, $1273(m), 1211(v s), 1070(m), 750(m), 697(m)$.

HRMS (ESI) calc. for $\mathrm{C}_{14} \mathrm{H}_{16} \mathrm{NaO}_{2} \mathrm{~S}[\mathrm{M}+\mathrm{Na}]^{+}:$271.0763; found: 271.0756 .

\section{Dihydrothiophene 12e}

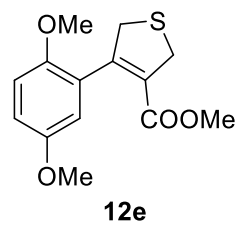

Following the General Procedures GP1 (14 kbar) and GP2, methyl propiolate S-41 (110 mg, $0.50 \mathrm{mmol}$ ) was converted into dihydrothiophene 12e. Purification by flash-column chromatography on silica gel (30\% dichloromethane in pentane) furnished 12e (GP1: $68 \mathrm{mg}$, 49\%; GP2: 20 mg, 14\%) as yellowish solid. Recrystallization from diethyl ether gave crystals suitable for X-ray diffraction.

$\operatorname{TLC}\left(50 \%\right.$ dichloromethane in pentane): $R_{\mathrm{f}}=0.31\left(\mathrm{UV}, \mathrm{KMnO}_{4}\right)$.

${ }^{1} \mathrm{H} \mathrm{NMR}\left(\mathrm{CDCl}_{3}, 400 \mathrm{MHz}\right) \delta=6.84-6.80(\mathrm{~m}, 2 \mathrm{H}), 6.66(\mathrm{t}, J=1.8 \mathrm{~Hz}, 1 \mathrm{H}), 4.18-4.13(\mathrm{~m}$, $2 \mathrm{H}), 4.13-4.09(\mathrm{~m}, 2 \mathrm{H}), 3.75(\mathrm{~s}, 3 \mathrm{H}), 3.74(\mathrm{~s}, 3 \mathrm{H}), 3.57(\mathrm{~s}, 3 \mathrm{H})$. 
${ }^{13} \mathrm{C}$ NMR $\left(\mathrm{CDCl}_{3}, 101 \mathrm{MHz}\right) \delta=164.7,153.4,150.2,149.1,129.3,126.2,114.9,114.0$, 112.0, 56.2, 55.8, 51.6, 43.9, 39.6.

IR (Diamond-ATR, $\left.\mathrm{CDCl}_{3}\right): \tilde{v}_{\max }=2947(w), 2833(w), 1726(m), 1498(m), 1276(m)$, $1216(s), 1118(m), 1043(m), 804(w), 729(w)$.

HRMS (ESI) calc. for $\mathrm{C}_{14} \mathrm{H}_{16} \mathrm{NaO}_{4} \mathrm{~S}[\mathrm{M}+\mathrm{Na}]^{+}:$303.0662; found: 303.0653 .

MP: $83-85^{\circ} \mathrm{C}$.

\section{Thiophenes $12 \mathrm{f}$ and $12 \mathrm{~g}$}

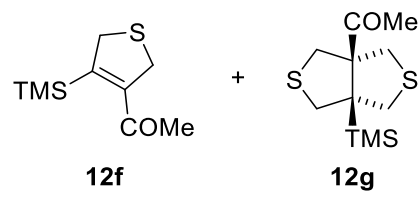

Following the General Procedures GP1 (14 kbar) and GP2, 4-trimethylsilyl-3-butyn-2-one (70 mg, $0.50 \mathrm{mmol}$ ) was converted into thiophenes $12 \mathrm{f}$ and $\mathbf{1 2 g}$. Purification by flash-column chromatography on silica gel (25\% dichloromethane in pentane) furnished 12f (GP1: $21 \mathrm{mg}$, 21\%; GP2: 10 mg, 10\%) and 12g (GP1: 10 mg, 8\%; GP2: not detected) as colorless oils.

\section{Dihydrothiophene 12f:}

TLC (50\% dichloromethane in pentane): $\mathrm{R}_{f}=0.38\left(\mathrm{UV}, \mathrm{KMnO}_{4}\right)$

${ }^{1} \mathrm{H}$ NMR $\left(\mathrm{CDCl}_{3}, 400 \mathrm{MHz}\right): \delta=4.14-4.09(\mathrm{~m}, 2 \mathrm{H}), 4.05-4.00(\mathrm{~m}, 2 \mathrm{H}), 2.25(\mathrm{~s}, 3 \mathrm{H}), 0.16$ $(\mathrm{s}, 9 \mathrm{H})$.

${ }^{13} \mathrm{C}$ NMR $\left(\mathrm{CDCl}_{3}, 101 \mathrm{MHz}\right): \delta=196.5,157.2,148.3,46.1,42.0,29.7,-0.9$.

IR (Diamond-ATR, $\left.\mathrm{CDCl}_{3}\right): \tilde{v}_{\max }=2951(w), 2900(w), 1685(s), 1578(m), 1357(m), 1246(s)$, $1204(m), 839(v s), 761(m), 616(m)$.

HRMS (ESI) calc. for $\mathrm{C}_{9} \mathrm{H}_{16} \mathrm{NaOSSi}[\mathrm{M}+\mathrm{Na}]^{+}:$223.0583; found: 223.0581.

Tetrahydrothiophene 12g:

$\operatorname{TLC}\left(50 \%\right.$ dichloromethane in pentane): $\mathrm{R}_{f}=0.33\left(\mathrm{UV}, \mathrm{KMnO}_{4}\right)$

${ }^{1} \mathbf{H}$ NMR $\left(\mathrm{CDCl}_{3}, 400 \mathrm{MHz}\right): \delta=3.28(\mathrm{~d}, J=11.5 \mathrm{~Hz}, 2 \mathrm{H}), 3.20-3.13(\mathrm{~m}, 4 \mathrm{H}), 3.02(\mathrm{~d}$, $J=11.1 \mathrm{~Hz}, 2 \mathrm{H}), 2.34(\mathrm{~s}, 3 \mathrm{H}), 0.16(\mathrm{~s}, 9 \mathrm{H})$.

${ }^{13} \mathrm{C}$ NMR $\left(\mathrm{CDCl}_{3}, 101 \mathrm{MHz}\right) \delta=207.9,76.1,51.5,39.8,39.1,27.5,-0.8$. 
IR (Diamond-ATR, $\left.\mathrm{CDCl}_{3}\right): \widetilde{v}_{\max }=2949(m), 1708(s), 1453(w), 1356(m), 1249(s), 1175(w)$, $1129(w), 841(v s), 759(m), 688(w)$.

HRMS (ESI) calc. for $\mathrm{C}_{11} \mathrm{H}_{20}{ }^{107} \mathrm{AgOS} \mathrm{Si}\left[\mathrm{M}+{ }^{107} \mathrm{Ag}\right]^{+}: 366.9770$; found: 366.9760 .

\subsubsection{Limitations}

- Reactivity and Selectivity<smiles>O=CC1=C(c2ccccc2)CSC1</smiles>

S-43ab

$48 \%, 1.4: 1(-1 \%, 1: 1.3)$

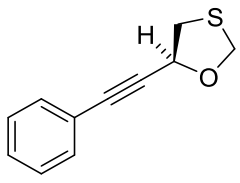

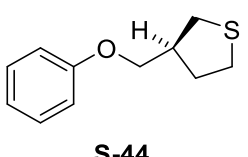

S-44

$30 \%(+30 \%)$

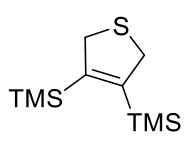

S-45

$34 \%(+19 \%)$

- Purification

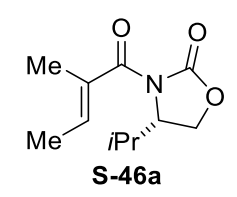

(mixture of diastereomers)<smiles>CCOC(=O)C=C1CCC1</smiles>

S-46b

(copolarity)

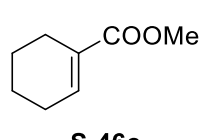

S-46c

(copolarity)

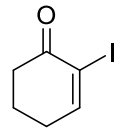

s-46d

(complex mixture)

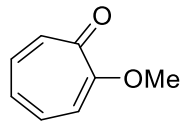

S-46e

(complex mixture)

_ Unreactive Substrates<smiles>COC1=CC(=O)CCC1</smiles>

s-47a<smiles>C=C(C)c1ccccc1</smiles>

S-47h<smiles>Oc1ccccc1</smiles>

S-47b<smiles>C/C=C\c1ccccc1</smiles>

S-47i<smiles>CCOC(=O)C=C1CCCC1</smiles>

S-47c<smiles>C#Cc1ccccc1</smiles>

S-47j<smiles>CCOC(=O)C=C1CCCCC1</smiles>

S-47d<smiles>C1=CCCCC1</smiles>

S-47e<smiles>C1=COCCC1</smiles>

S-47f<smiles>C=C([OH2+])c1ccccc1</smiles>

S-47g<smiles>C#COc1ccccc1</smiles>

S-47k<smiles>O=Cc1c[nH]c2ccccc12</smiles><smiles>Cn1cc(C=O)c2ccccc21</smiles>

Figure 2: Limitations of the high-pressure mediated cycloaddition of sulfoxide 1a. 


\section{Dihydrothiophene S-43a and 1,3-Oxathiolane S-43b}
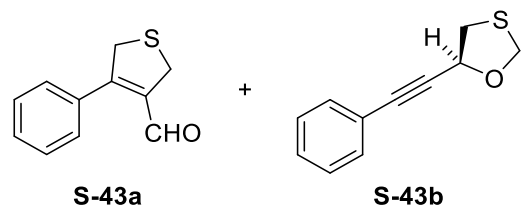

Following the General Procedures GP1 (14 kbar) and GP2, 3-phenylpropiolaldehyde (65 mg,

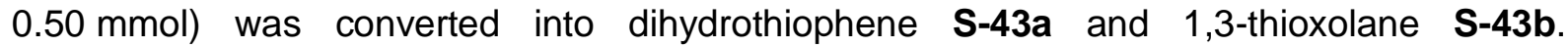
Purification by flash-column chromatography on silica gel (20\% toluene in cyclohexane) furnished S-43a (GP1: $27 \mathrm{mg}, 28 \%$; GP2: $20 \mathrm{mg}, 21 \%$ ) as a yellowish oil and S-43b (GP1: 19 mg, 20\%; GP2: 27 mg, 28\%) as a colorless oil.

\section{Dihydrothiophene S-43a:}

TLC (10\% ethyl acetate in cyclohexane): $\mathrm{R}_{f}=0.39\left(\mathrm{UV}, \mathrm{KMnO}_{4}\right)$.

${ }^{1} \mathrm{H}$ NMR $\left(\mathrm{CDCl}_{3}, 400 \mathrm{MHz}\right): \delta=9.64(\mathrm{~s}, 1 \mathrm{H}), 7.46-7.42(\mathrm{~m}, 3 \mathrm{H}), 7.34(\mathrm{~d}, J=2.3 \mathrm{~Hz}, 2 \mathrm{H})$, 4.32 (dd, $J=4.9,3.8 \mathrm{~Hz}, 2 \mathrm{H}), 4.11$ (dd, $J=4.8,3.8 \mathrm{~Hz}, 2 \mathrm{H}$ ).

${ }^{13} \mathrm{C}$ NMR $\left(\mathrm{CDCl}_{3}, 101 \mathrm{MHz}\right): \delta=189.7,159.7,137.9,133.5,130.0,128.9,128.8,44.7,37.6$.

IR (Diamond-ATR, $\mathrm{CDCl}_{3}$ ): $\tilde{v}_{\max }=2918(w), 2846(v s), 2188(m), 1660(v s), 1624(m)$, $1328(m), 1176(m), 767(s), 740(m), 699(s)$.

HRMS (ESI) calc. for $\mathrm{C}_{11} \mathrm{H}_{11} \mathrm{OS}[\mathrm{M}+\mathrm{H}]^{+}:$191.0525; found: 191.0520 .

\section{1,3-Oxathiolane S-43b:}

TLC (toluene): $\mathrm{R}_{f}=0.26\left(\mathrm{UV}, \mathrm{KMnO}_{4}\right)$

${ }^{1} \mathrm{H}$ NMR $\left(\mathrm{CDCl}_{3}, 400 \mathrm{MHz}\right): \delta=7.48-7.43(\mathrm{~m}, 2 \mathrm{H}), 7.36-7.29(\mathrm{~m}, 3 \mathrm{H}), 5.11-5.06(\mathrm{~m}$, 2H), $4.99-4.96(\mathrm{~m}, 1 \mathrm{H}), 3.31(\mathrm{dd}, J=10.1,6.0 \mathrm{~Hz}, 1 \mathrm{H}), 3.18(\mathrm{dd}, J=10.1,5.5 \mathrm{~Hz}, 1 \mathrm{H})$.

${ }^{13} \mathrm{C}$ NMR $\left(\mathrm{CDCl}_{3}, 101 \mathrm{MHz}\right): \delta=132.0,128.9,128.4,122.1,86.2,85.2,72.3,71.0,38.0$.

IR (Diamond-ATR, $\left.\mathrm{CDCl}_{3}\right): \tilde{v}_{\max }=2941(w), 2869(w), 2234(w), 1489(s), 1442(m)$, $1333(m), 1052$ (vs), $959(m), 756(v s), 691(v s)$.

HRMS (ESI) calc. for $\mathrm{C}_{11} \mathrm{H}_{10} \mathrm{NaOS}$ [M+Na] $]^{+}: 213.0345$; found: 213.0340 . 


\section{Tetrahydrothiophene S-44}

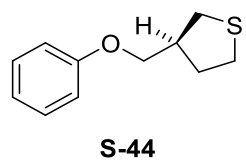

Following the General Procedures GP1 (14 kbar) and GP2, allyl phenyl ether $(67 \mathrm{mg}$, $0.50 \mathrm{mmol}$ ) was converted into tetrahydrothiophene $\mathbf{S - 4 4}$. Purification by flash-column chromatography on silica gel (1\% diethyl ether in pentane) furnished S-44 (GP1: $29 \mathrm{mg}$, $30 \%$; GP2: not detected) as a colorless oil.

TLC (1\% diethyl ether in pentane): $R_{\mathrm{f}}=0.33\left(\mathrm{UV}, \mathrm{KMnO}_{4}\right)$.

${ }^{1} \mathrm{H}$ NMR $\left(\mathrm{CDCl}_{3}, 400 \mathrm{MHz}\right): \delta=7.31-7.26(\mathrm{~m}, 2 \mathrm{H}), 6.95(\mathrm{tt}, J=7.3,1.1 \mathrm{~Hz}, 1 \mathrm{H}), 6.92-6.88$ $(\mathrm{m}, 2 \mathrm{H}), 3.95(\mathrm{~d}, J=6.5 \mathrm{~Hz}, 2 \mathrm{H}), 3.07-3.01(\mathrm{~m}, 1 \mathrm{H}), 2.95-2.89(\mathrm{~m}, 2 \mathrm{H}), 2.80-2.68(\mathrm{~m}$, 2H), 2.22 (ddt, $J=12.0,6.2,5.4 \mathrm{~Hz}, 1 \mathrm{H}), 1.90(\mathrm{dq}, J=13.1,7.9 \mathrm{~Hz}, 1 \mathrm{H})$.

${ }^{13} \mathrm{C}$ NMR $\left(\mathrm{CDCl}_{3}, 101 \mathrm{MHz}\right): \delta=159.0,129.6,121.0,114.6,69.4,44.0,34.1,33.7,30.8$.

IR (Diamond-ATR, $\left.\mathrm{CDCl}_{3}\right): \tilde{v}_{\max }=2931(w), 1598(m), 1586(w), 1495(m), 1467(m)$, $1238(v s), 1171(w), 1036(m), 752(s), 690(s)$.

HRMS (ESI) calc. for $\mathrm{C}_{11} \mathrm{H}_{14}{ }^{107} \mathrm{AgOS}\left[\mathrm{M}+{ }^{107} \mathrm{Ag}\right]^{+}: 300.9811$; found: 300.9797 .

\section{Dihydrothiophene S-45}

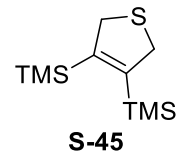

Following the General Procedures GP1 (14 kbar), bis(trimethylsilyl)acetylene (85 mg, $0.50 \mathrm{mmol}$ ) was converted into dihydrothiophene S-45. Purification by flash-column chromatography on silica gel (pentane) furnished S-45 (GP1: $39 \mathrm{mg}, 43 \%$ ) as colorless liquid. The obtained analytical data was in full agreement with those reported in literature. ${ }^{22}$

TLC (pentane): $\mathrm{R}_{f}=0.21\left(\mathrm{UV}, \mathrm{KMnO}_{4}\right)$.

${ }^{1} \mathrm{H}$ NMR $\left(\mathrm{CDCl}_{3}, 400 \mathrm{MHz}\right) \delta=4.06(\mathrm{~s}, 4 \mathrm{H}), 0.21(\mathrm{~s}, 18 \mathrm{H})$.

${ }^{13} \mathrm{C} \mathrm{NMR}\left(\mathrm{CDCl}_{3}, 101 \mathrm{MHz}\right) \delta=150.8,50.6,1.0$.

IR (Diamond-ATR, $\mathrm{CDCl}_{3}$ ): $\tilde{v}_{\max }=2953(w), 2897(w), 1558(w), 1248(s), 985(m), 904(s)$, $824(v s), 752(s), 688(m), 624(m)$. 
HRMS (ESI) calc. for $\mathrm{C}_{10} \mathrm{H}_{22}{ }^{107} \mathrm{AgSSi}_{2}\left[\mathrm{M}+{ }^{107} \mathrm{Ag}\right]^{+}:$: 337.0026; found: 337.0021 .

Note: An experiment following GP2 was not conducted since there is a reported yield for the thermal [3+2]-cycloaddition yielding S-45 using hexamethylphosphoramide as solvent (yield: 15\%). ${ }^{22}$ 


\subsection{Postfunctionalizations}

\section{Ketone 13}

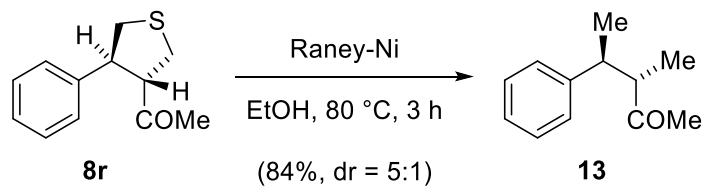

To a suspension of Raney ${ }^{\circledR}$-Nickel (0.50 g, W.R. Grace and Co. Raney ${ }^{\circledR}$ 2800, slurry in water) in ethanol ( $4 \mathrm{~mL}$ ) was added tetrahydrothiophene $8 \mathrm{r}$ (50 mg, $0.24 \mathrm{mmol}, 1$ equiv) in one portion at $23^{\circ} \mathrm{C}$. Upon addition, the mixture was heated to $80^{\circ} \mathrm{C}$. After three hours, the suspension was filtered through a plug of Celite $^{\circledR}$ and the filtrate was concentrated. The crude product was purified by flash-column chromatography on silica gel (20\% ethyl acetate in cyclohexane) to furnish ketone 13 (36 $\mathrm{mg}, 84 \%$, inseparable mixture of two diastereomers, $d r=5: 1)$ as a colorless oil. The obtained analytical data for both diastereomers were in full agreement with those reported in literature. ${ }^{23}$

TLC (20\% diethyl ether in pentane): $R_{\mathrm{f}}=0.57\left(\mathrm{UV}, \mathrm{KMnO}_{4}\right)$

\section{Major diastereomer:}

${ }^{1} \mathbf{H}$ NMR $\left(\mathrm{CDCl}_{3}, 400 \mathrm{MHz}\right): \delta=7.32-7.26(\mathrm{~m}, 2 \mathrm{H}), 7.21-7.15(\mathrm{~m}, 3 \mathrm{H}), 3.02(\mathrm{dq}, J=8.8$, $7.0 \mathrm{~Hz}, 1 \mathrm{H}), 2.79(\mathrm{dq}, J=8.7,6.9 \mathrm{~Hz}, 1 \mathrm{H}), 1.88(\mathrm{~s}, 3 \mathrm{H}), 1.25(\mathrm{~d}, J=7.0 \mathrm{~Hz}, 3 \mathrm{H}), 1.11(\mathrm{~d}, J=$ $6.9 \mathrm{~Hz}, 3 \mathrm{H})$.

${ }^{13} \mathrm{C}$ NMR $\left(\mathrm{CDCl}_{3}, 101 \mathrm{MHz}\right): \delta=212.5,145.4,128.6,127.4,126.5,53.9,42.0,29.5,18.0$, 13.9.

HRMS (ESI) calc. for $\mathrm{C}_{12} \mathrm{H}_{16} \mathrm{NaO}[\mathrm{M}+\mathrm{Na}]^{+}:$199.1093; found: 199.1093.

\section{Sulfoxide 14}

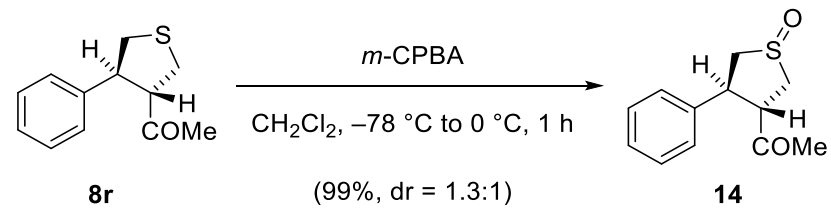

To a solution of tetrahydrothiophene $8 \mathrm{r} \quad(50 \mathrm{mg}, \quad 0.24 \mathrm{mmol}, \quad 1$ equiv) in dichloromethane $(1 \mathrm{~mL})$ was added a solution of meta-chloroperbenzoic acid $(61 \mathrm{mg}$, $0.27 \mathrm{mmol}, 1.1$ equiv) in dichloromethane $(1 \mathrm{~mL})$ dropwise at $-78^{\circ} \mathrm{C}$. Upon addition, the reaction mixture was allowed to warm to $0^{\circ} \mathrm{C}$. After one hour, water $(10 \mathrm{~mL})$ and dichloromethane $(5 \mathrm{~mL})$ were added, the layers separated and the aqueous layer was 
extracted with dichloromethane $(2 \times 7 \mathrm{~mL})$. The combined organic layers were washed with saturated aqueous bicarbonate solution $(30 \mathrm{~mL})$, dried over sodium sulfate, filtered and concentrated to furnish sulfoxide 14 (54 $\mathrm{mg}, 99 \%, \mathrm{dr}=1.3: 1)$ as a colorless solid.

$\operatorname{TLC}\left(5 \%\right.$ methanol in dichloromethane): $R_{f}=0.49\left(\mathrm{UV}, \mathrm{KMnO}_{4}\right)$.

Major diastereomer:

${ }^{1} \mathrm{H}$ NMR $\left(\mathrm{CDCl}_{3}, 400 \mathrm{MHz}\right): \delta=7.44-7.22(\mathrm{~m}, 5 \mathrm{H}), 4.15$ (ddd, $\left.J=12.8,10.4,5.3 \mathrm{~Hz}, 1 \mathrm{H}\right)$, $3.80(\mathrm{dd}, J=14.6,10.2 \mathrm{~Hz}, 1 \mathrm{H}), 3.69-3.57(\mathrm{~m}, 1 \mathrm{H}), 3.29$ (ddd, $J=13.5,5.3,2.4 \mathrm{~Hz}, 1 \mathrm{H}$ ), 2.94 (ddd, $J=15.0,7.4,2.6 \mathrm{~Hz}, 1 \mathrm{H}), 2.90-2.84(\mathrm{~m}, 1 \mathrm{H}), 1.99$ (s, 3H).

${ }^{13} \mathrm{C}$ NMR $\left(\mathrm{CDCl}_{3}, 101 \mathrm{MHz}\right): \delta=206.9,140.3,129.2,128.0,127.3,61.9,59.4,55.8,49.7$, 31.3.

Minor diastereomer:

${ }^{1} \mathrm{H}$ NMR $\left(\mathrm{CDCl}_{3}, 400 \mathrm{MHz}\right): \delta=7.44-7.22(\mathrm{~m}, 5 \mathrm{H}), 4.32(\mathrm{ddd}, J=12.6,10.3,5.7 \mathrm{~Hz}, 1 \mathrm{H})$, $3.70-3.58(\mathrm{~m}, 2 \mathrm{H}), 3.42-3.32(\mathrm{~m}, 1 \mathrm{H}), 3.20$ (ddd, $J=14.2,7.7,2.1 \mathrm{~Hz}, 1 \mathrm{H}), 2.91-2.77$ $(\mathrm{m}, 1 \mathrm{H}), 2.01(\mathrm{~s}, 3 \mathrm{H})$.

${ }^{13} \mathrm{C}$ NMR $\left(\mathrm{CDCl}_{3}, 101 \mathrm{MHz}\right): \delta=205.2,138.4,129.3,128.0,127.6,60.1,59.0,56.4,48.1$, 29.4 .

IR (Diamond-ATR, $\left.\mathrm{CDCl}_{3}\right): \tilde{v}_{\max }=3444(w), 1707(s), 1407(m), 1359(w), 1169(w)$, $1016(v s), 768(m), 754(m), 703(s), 523(m)$.

HRMS (ESI) calc. for $\mathrm{C}_{12} \mathrm{H}_{14} \mathrm{NaO}_{2} \mathrm{~S}[\mathrm{M}+\mathrm{Na}]^{+}: 245.0607$; found: 245.0598 .

MP $=47-49^{\circ} \mathrm{C}$.

\section{Sulfolane 15}

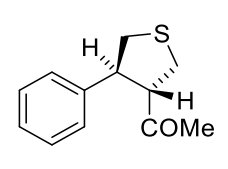

$8 \mathbf{r}$

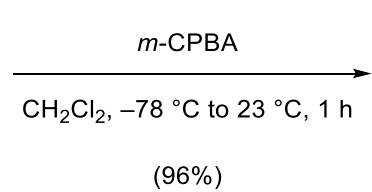

(96\%)

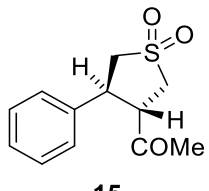

15

To a solution of tetrahydrothiophene $8 \mathrm{r} \quad(240 \mathrm{mg}, \quad 1.16 \mathrm{mmol}, \quad 1$ equiv) in dichloromethane $(5 \mathrm{~mL})$ was added a solution of meta-chloroperbenzoic acid $(590 \mathrm{mg}$, $2.56 \mathrm{mmol}, 2.20$ equiv) in dichloromethane $(5 \mathrm{~mL})$ dropwise at $-78{ }^{\circ} \mathrm{C}$. Upon addition, the reaction mixture was allowed to warm to $23^{\circ} \mathrm{C}$. After one hour, water $(20 \mathrm{~mL})$ and 
dichloromethane $(10 \mathrm{~mL})$ were added, the layers separated, and the aqueous layer was extracted with dichloromethane $(2 \times 10 \mathrm{~mL})$. The combined organic layers were washed with saturated aqueous bicarbonate solution $(30 \mathrm{~mL})$, dried over sodium sulfate, filtered and concentrated to furnish sulfolane 15 (266 $\mathrm{mg}, 96 \%$ ) as a colorless solid.

TLC (30\% ethyl acetate in cyclohexane): $R_{\mathrm{f}}=0.38\left(\mathrm{UV}, \mathrm{KMnO}_{4}\right)$.

${ }^{1} \mathbf{H}$ NMR $\left(\mathrm{CDCl}_{3}, 400 \mathrm{MHz}\right): \delta=7.41-7.34(\mathrm{~m}, 2 \mathrm{H}), 7.34-7.28(\mathrm{~m}, 3 \mathrm{H}), 3.75-3.56(\mathrm{~m}$, $3 \mathrm{H}), 3.48-3.36(\mathrm{~m}, 2 \mathrm{H}), 3.27$ (ddt, $J=12.7,9.2,0.9 \mathrm{~Hz}, 1 \mathrm{H}), 1.87$ (s, 3H).

${ }^{13} \mathrm{C}$ NMR $\left(\mathrm{CDCl}_{3}, 101 \mathrm{MHz}\right): \delta=204.5,138.0,129.5,128.5,127.3,58.6,54.4,54.4,45.0$, 31.3.

IR (Diamond-ATR, $\mathrm{CDCl}_{3}$ ): $\tilde{v}_{\max }=1715(\mathrm{vs}), 1307(\mathrm{~m}), 1179(\mathrm{~m}), 1146(\mathrm{~m}), 1117(\mathrm{~m})$, $875(w), 736(m), 698(w), 527(w), 448(w)$.

HRMS (ESI) calc. for $\mathrm{C}_{12} \mathrm{H}_{14} \mathrm{NaO}_{3} \mathrm{~S}[\mathrm{M}+\mathrm{Na}]^{+}:$261.0556; found: 261.0547 .

$M P=134-136^{\circ} \mathrm{C}$.

\section{Enone 16}

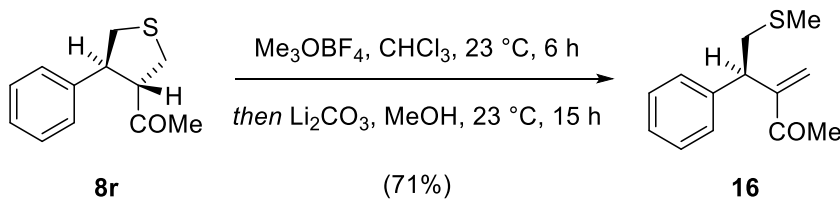

To a solution of dihydrothiophene $8 \mathrm{r}$ (100 mg, $485 \mu \mathrm{mol}, 1$ equiv) in chloroform (6 mL) was added trimethyloxonium tetrafluoroborate (113 mg, $727 \mu \mathrm{mol}, 1.50$ equiv) in one portion at $23^{\circ} \mathrm{C}$. After six hours, the obtained suspension was concentrated. The residue was dissolved in methanol $(6 \mathrm{~mL})$ and lithium carbonate $(107 \mathrm{mg}, 1.45 \mathrm{mmol}, 3.00$ equiv) was added at $23{ }^{\circ} \mathrm{C}$. After 15 hours, saturated aqueous ammonium chloride solution ( $10 \mathrm{~mL}$ ) was added, the layers separated and the aqueous layer extracted with diethyl ether $(3 \times 50 \mathrm{~mL})$. The combined organic layers were dried over sodium sulfate, filtered and concentrated. The crude product was purified by flash-column chromatography on silica gel (10\% ethyl acetate in cyclohexane) to furnish enone $16(76.0 \mathrm{mg}, 71 \%)$ as a colorless oil.

TLC $\left(10 \%\right.$ ethyl acetate in cyclohexane): $R_{\mathrm{f}}=0.35\left(\mathrm{UV}, \mathrm{KMnO}_{4}\right)$.

${ }^{1}$ H NMR $\left(\mathrm{CDCl}_{3}, 400 \mathrm{MHz}\right): \delta=7.34-7.20(\mathrm{~m}, 5 \mathrm{H}), 6.23,(\mathrm{~s}, 1 \mathrm{H}), 5.86(\mathrm{~d}, J=1.0 \mathrm{~Hz}, 1 \mathrm{H})$, $4.33(\mathrm{dt}, J=7.8,0.9 \mathrm{~Hz}, 1 \mathrm{H}) .3 .03-2.88(\mathrm{~m}, 2 \mathrm{H}), 2.34(\mathrm{~s}, 3 \mathrm{H}), 2.08(\mathrm{~s}, 3 \mathrm{H})$. 
${ }^{13} \mathrm{C} \mathrm{NMR}\left(\mathrm{CDCl}_{3}, 101 \mathrm{MHz}\right): \delta=198.9,150.7,141.8,128.6,128.2,126.9,125.5,44.1,38.9$, 26.4, 16.1 .

IR (Diamond-ATR, $\left.\mathrm{CDCl}_{3}\right): \tilde{v}_{\max }=2916(\mathrm{w}), 1674(\mathrm{~s}), 1493(\mathrm{~m}), 1426(\mathrm{~m}), 1362(\mathrm{~m})$, $1114(\mathrm{~m}), 942(\mathrm{~m}), 733(\mathrm{~m}), 699(\mathrm{~s}), 545(\mathrm{~m})$.

HRMS (ESI) calc. for $\mathrm{C}_{13} \mathrm{H}_{16} \mathrm{NaOS}[\mathrm{M}+\mathrm{Na}]^{+}:$243.0820; found: 243.0806 .

\section{Thiophene 17}

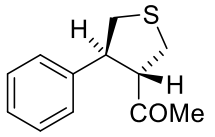

$8 r$

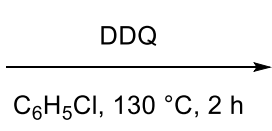

$(63 \%)$

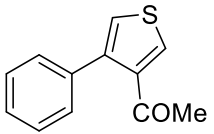

17

To a solution of tetrahydrothiophene $8 \mathrm{r} \quad(50 \mathrm{mg}, \quad 0.24 \mathrm{mmol}, 1$ equiv) in chlorobenzene (3.5 mL) under argon was added 2,3-dichloro-5,6-dicyano-1,4-benzochinone $\left(0.14 \mathrm{~g}, 0.61 \mathrm{mmol}, 2.5\right.$ equiv) in one portion at $23^{\circ} \mathrm{C}$. Upon addition, the mixture was heated to $130^{\circ} \mathrm{C}$. After two hours, the brownish suspension was filtered and concentrated. The crude product was purified by flash-column chromatography on silica gel (20\% ethyl acetate in cyclohexane) to furnish thiophene 17 (31 $\mathrm{mg}, 63 \%)$ as a yellow oil.

TLC (30\% ethyl acetate in cyclohexane): $R_{\mathrm{f}}=0.44\left(\mathrm{UV}, \mathrm{KMnO}_{4}\right)$.

${ }^{1} \mathbf{H}$ NMR $\left(\mathrm{CDCl}_{3}, 400 \mathrm{MHz}\right): \delta=8.01(\mathrm{~d}, J=3.3 \mathrm{~Hz}, 1 \mathrm{H}), 7.42-7.32(\mathrm{~m}, 5 \mathrm{H}), 7.18(\mathrm{~d}, J=$ $3.3 \mathrm{~Hz}, 1 \mathrm{H}), 2.32(\mathrm{~s}, 3 \mathrm{H})$.

${ }^{13} \mathrm{C}$ NMR $\left(\mathrm{CDCl}_{3}, 101 \mathrm{MHz}\right): \delta=194.5,142.9,141.3,136.5,133.2,129.0,128.2,127.7$, 125.2, 29.4 .

IR (Diamond-ATR, $\left.\mathrm{CDCl}_{3}\right): \tilde{v}_{\max }=3098(w), 1675(\mathrm{vs}), 1486(\mathrm{~m}), 1426(\mathrm{~m}), 1366(\mathrm{~m})$, $1222(s), 928(w), 808(s), 769(v s), 769(m)$.

HRMS (ESI) calc. for $\mathrm{C}_{12} \mathrm{H}_{10} \mathrm{NaOS}$ [M+Na] $]^{+}$:225.0345; found: 225.0344 . 


\section{Thiophene 17}

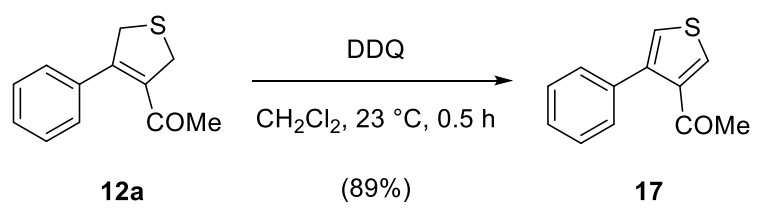

To a solution of dihydrothiophene $12 \mathrm{a} \quad(41 \mathrm{mg}, \quad 0.20 \mathrm{mmol}, \quad 1$ equiv) in dichloromethane $(2.5 \mathrm{~mL})$ under argon was added 2,3-dichloro-5,6-dicyano-1,4benzochinone (50 mg, $0.22 \mathrm{mmol}, 1.1$ equiv) in one portion at $23^{\circ} \mathrm{C}$. After 30 minutes, the orange suspension was filtered and concentrated. The crude product was purified by flashcolumn chromatography on silica gel (20\% ethyl acetate in cyclohexane) to furnish thiophene 17 (36 mg, 89\%) as a yellow oil. The obtained analytical data were in full agreement with those obtained for the oxidation of tetrahydrothiophene $8 \mathrm{r}$.

\section{Sulfolene 18}

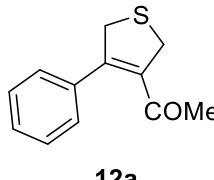

$12 \mathrm{a}$

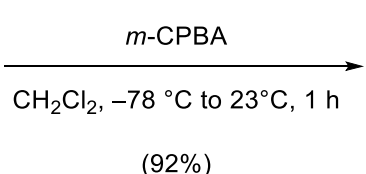

$(92 \%)$

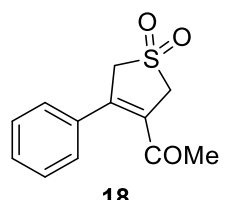

18

To a solution of dihydrothiophene $12 \mathrm{a}(50 \mathrm{mg}, 0.24 \mathrm{mmol}, 1$ equiv) in dichloromethane $(1 \mathrm{~mL})$ was added a solution of meta-chloroperbenzoic acid $(0.14 \mathrm{~g}, 0.61 \mathrm{mmol}, 2.5$ equiv) in dichloromethane $(1 \mathrm{~mL})$ dropwise at $-78^{\circ} \mathrm{C}$. Upon addition, the reaction mixture was allowed to warm to $23^{\circ} \mathrm{C}$. After one hour, water $(10 \mathrm{~mL})$ was added, the layers separated, and the aqueous layer was extracted with dichloromethane $(3 \times 10 \mathrm{~mL})$. The combined organic layers were washed with saturated aqueous sodium bicarbonate solution $(3 \times 10 \mathrm{~mL})$, dried over sodium sulfate, filtered and concentrated. The obtained residue was purified by flashcolumn chromatography on silica gel (1\% methanol in dichloromethane) to furnish sulfolene 18 (53 mg, 92\%) as a colorless solid.

TLC (1\% methanol in dichloromethane): $R_{\mathrm{f}}=0.53\left(\mathrm{UV}, \mathrm{KMnO}_{4}\right)$

${ }^{1} \mathrm{H}$ NMR $\left(\mathrm{CDCl}_{3}, 400 \mathrm{MHz}\right): \delta=7.26-7.23(\mathrm{~m}, 3 \mathrm{H}), 7.09-7.01(\mathrm{~m}, 2 \mathrm{H}), 4.08(\mathrm{t}, J=1.5 \mathrm{~Hz}$, $2 \mathrm{H}), 4.02(\mathrm{t}, J=1.5 \mathrm{~Hz}, 2 \mathrm{H}), 1.65(\mathrm{~s}, 3 \mathrm{H})$.

${ }^{13} \mathrm{C} \mathrm{NMR}\left(\mathrm{CDCl}_{3}, 101 \mathrm{MHz}\right): \delta=196.8,143.6,134.4,132.9,130.2,129.4,127.6,62.9,58.2$, 30.0 . 
IR (Diamond-ATR, $\mathrm{CDCl}_{3}$ ): $\tilde{v}_{\max }=2976(w), 2927(w), 1665(s), 1307$ (vs), 1233 (vs), 1123 (vs), 944 (m), 764 (vs), 698 (s), 442 (s).

HRMS (ESI) calc. for $\mathrm{C}_{12} \mathrm{H}_{12} \mathrm{NaO}_{3} \mathrm{~S}$ [M+Na] $]^{+}$:259.0399; found: 259.0397 .

MP: $101-103^{\circ} \mathrm{C}$.

\section{Diels-Alder adduct 20}
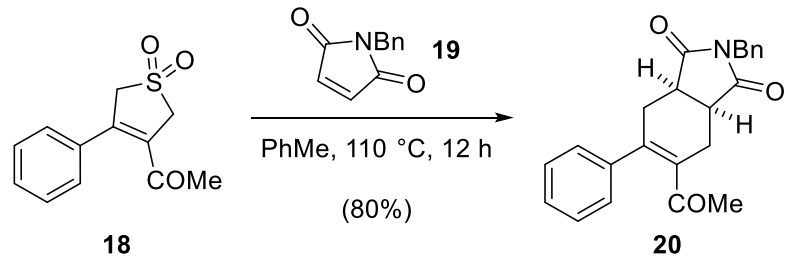

To a solution of sulfolene 18 (45 mg, $0.19 \mathrm{mmol}, 1$ equiv) in toluene (2 mL) was added $\mathrm{N}$-benzylmaleimide 19 (71 $\mathrm{mg}, 0.38 \mathrm{mmol}, 2.0$ equiv) in one portion. The reaction mixture was heated to $110^{\circ} \mathrm{C}$. After twelve hours, the solvent was removed and the crude product was purified by flash-column chromatography on silica gel $(30 \%$ ethyl acetate in cyclohexane) to furnish Diels-Alder adduct $20(55 \mathrm{mg}, 80 \%)$ as a colorless solid. Recrystallization from diethyl ether gave crystals suitable for $\mathrm{X}$-ray diffraction.

$\operatorname{TLC}\left(30 \%\right.$ ethyl acetate in cyclohexane): $R_{\mathrm{f}}=0.23\left(\mathrm{UV}, \mathrm{KMnO}_{4}\right)$.

${ }^{1} \mathrm{H}$ NMR $\left(\mathrm{CDCl}_{3}, 300 \mathrm{MHz}\right): \delta=7.49-7.07(\mathrm{~m}, 8 \mathrm{H}), 6.92-6.81(\mathrm{~m}, 2 \mathrm{H}), 4.61(\mathrm{~s}, 2 \mathrm{H}), 3.29-$ $3.17(\mathrm{~m}, 2 \mathrm{H}), 3.05(\mathrm{dd}, J=22.0,15.4 \mathrm{~Hz}, 2 \mathrm{H}), 2.64-2.50(\mathrm{~m}, 1 \mathrm{H}), 2.40-2.28(\mathrm{~m}, 1 \mathrm{H}), 1.24$ $(\mathrm{s}, 3 \mathrm{H})$.

${ }^{13} \mathrm{C}$ NMR $\left(\mathrm{CDCl}_{3}, 101 \mathrm{MHz}\right): \delta=203.4,179.3,178.9,145.2,139.9,136.5,136.0,129.1$, $128.7,128.7,128.7,128.1,127.8,42.9,39.8,39.4,32.6,29.5,26.6$.

IR (Diamond-ATR, $\left.\mathrm{CDCl}_{3}\right): \tilde{v}_{\max }=2928(w), 1693(v s), 1430(w), 1397(m), 1346(s)$, $1238(m), 1166(s), 917(m), 763(s), 700(v s)$.

HRMS (ESI) calc. for $\mathrm{C}_{23} \mathrm{H}_{21} \mathrm{NaNO}_{3}[\mathrm{M}+\mathrm{Na}]^{+}: 382.1414$; found: 382.1410 .

MP: $119-121^{\circ} \mathrm{C}$. 


\section{Diene 21}

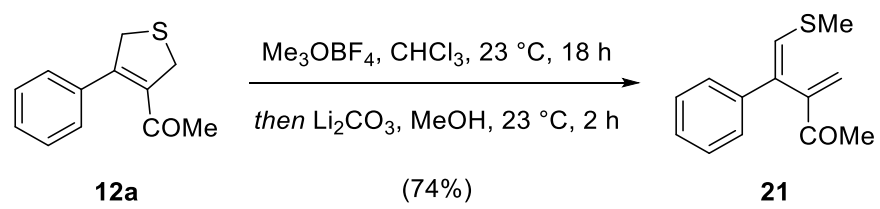

To a solution of dihydrothiophene $12 \mathrm{a}(150 \mathrm{mg}, 0.734 \mathrm{mmol}, 1$ equiv) in chloroform $(7.5 \mathrm{~mL})$ was added trimethyloxonium tetrafluoroborate $(171 \mathrm{mg}, 1.10 \mathrm{mmol}, 1.50$ equiv) in one portion at $23^{\circ} \mathrm{C}$. After 18 hours, the obtained suspension was concentrated. The residue was dissolved in methanol $(7.5 \mathrm{~mL})$ and lithium carbonate (109 mg, $1.47 \mathrm{mmol}, 2.00$ equiv) was added at $23{ }^{\circ} \mathrm{C}$. After two hours, saturated aqueous ammonium chloride solution $(10 \mathrm{~mL})$ was added, the layers separated and the aqueous layer extracted with diethyl ether $(3 \times 50 \mathrm{~mL})$. The combined organic layers were dried over sodium sulfate, filtered and concentrated. The crude product was purified by flash-column chromatography on silica gel (5\% diethyl ether in pentane) to furnish diene 21 (119 mg, 74\%) as an orange oil.

TLC (10\% diethyl ether in pentane): $R_{\mathrm{f}}=0.28\left(\mathrm{UV}, \mathrm{KMnO}_{4}\right)$.

${ }^{1} \mathrm{H}$ NMR $\left(\mathrm{CDCl}_{3}, 400 \mathrm{MHz}\right): \delta=7.25-7.13(\mathrm{~m}, 5 \mathrm{H}), 6.57(\mathrm{~s}, 1 \mathrm{H}), 6.35(\mathrm{~d}, J=1.2 \mathrm{~Hz}, 1 \mathrm{H})$, 5.69 (d, $J=1.2 \mathrm{~Hz}, 1 \mathrm{H}), 2.31(\mathrm{~s}, 3 \mathrm{H}), 2.20(\mathrm{~s}, 3 \mathrm{H})$.

${ }^{13} \mathrm{C}$ NMR $\left(\mathrm{CDCl}_{3}, 101 \mathrm{MHz}\right): \delta=198.7,146.7,139.3,135.1,129.4,128.7,128.6,127.2$, $125.5,27.1,17.5$.

IR (Diamond-ATR, $\left.\mathrm{CDCl}_{3}\right): \tilde{v}_{\max }=2922(w), 1694(s), 1685(s), 1422(w), 1356(m), 1141(w)$, $954(m), 820(m), 760(v s), 696(m)$.

HRMS (ESI) calc. for $\mathrm{C}_{13} \mathrm{H}_{14} \mathrm{NaOS}[\mathrm{M}+\mathrm{Na}]^{+}:$241.0658; found: 241.0648 .

\section{Dihydropyran 23}

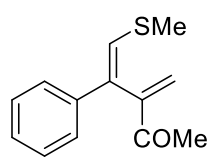

21

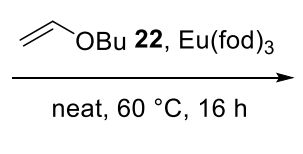

$(75 \%)$

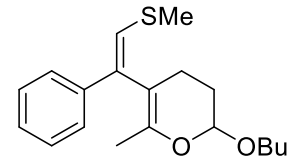

23

To a mixture of diene 21 (10 mg, $46 \mu \mathrm{mol}, 1$ equiv) and $n$-butyl vinyl ether $(22,0.20 \mathrm{~mL}$ ) was added tris(6,6,7,7,8,8,8-heptafluoro-2,2-dimethyl-3,5-octanedionato)europium (2.4 mg, $2.3 \mu \mathrm{mol}, 0.05$ equiv). Upon addition, the reaction mixture was heated to $60^{\circ} \mathrm{C}$. After 16 hours, the reaction was concentrated and the residue was purified by flash-column 
chromatography on silica gel (3\% diethyl ether in pentane) to furnish dihydropyran 23 (11 mg, 75\%) as a colorless oil.

TLC $\left(10 \%\right.$ diethyl ether in pentane): $R_{\mathrm{f}}=0.63$ (UV, CAM)

${ }^{1} \mathbf{H}$ NMR $\left(\mathrm{CDCl}_{3}, 400 \mathrm{MHz}\right): \delta=7.37-7.31(\mathrm{~m}, 2 \mathrm{H}), 7.31-7.26(\mathrm{~m}, 2 \mathrm{H}), 7.22-7.16(\mathrm{~m}$, $1 \mathrm{H}), 6.46(\mathrm{~s}, 1 \mathrm{H}), 5.06(\mathrm{t}, J=3.2 \mathrm{~Hz}, 1 \mathrm{H}), 3.87(\mathrm{dt}, J=9.6,6.7 \mathrm{~Hz}, 1 \mathrm{H}), 3.58(\mathrm{dt}, J=9.6$, $6.4 \mathrm{~Hz}, 1 \mathrm{H}), 2.37(\mathrm{~s}, 3 \mathrm{H}), 2.25-2.09(\mathrm{~m}, 1 \mathrm{H}), 1.96-1.86(\mathrm{~m}, 3 \mathrm{H}), 1.70(\mathrm{t}, J=1.7 \mathrm{~Hz}, 3 \mathrm{H})$, $1.68-1.59(\mathrm{~m}, 2 \mathrm{H}), 1.51-1.40(\mathrm{~m}, 2 \mathrm{H}), 0.96(\mathrm{t}, J=7.4 \mathrm{~Hz}, 3 \mathrm{H})$.

${ }^{13} \mathrm{C}$ NMR $\left(\mathrm{CDCl}_{3}, 101 \mathrm{MHz}\right): \delta=145.7,139.24,136.4,128.6,127.4,126.7,125.4,106.8$, $97.3,68.0,32.2,27.2,20.1,19.6,17.3,17.2,14.1$.

IR (Diamond-ATR, $\left.\mathrm{CDCl}_{3}\right): \tilde{v}_{\max }=2919(m), 2870(m), 1673(m), 1441(w), 1243(m)$, $1097(s), 1060(s), 866(m), 754(s), 694(s)$.

HRMS (ESI) calc. for $\mathrm{C}_{19} \mathrm{H}_{26} \mathrm{NaO}_{2} \mathrm{~S}$ [M+Na] $]^{+}$341.1546; found: 341.1529 .

\section{[3]Dendralene 24}

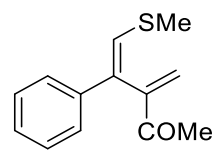

21

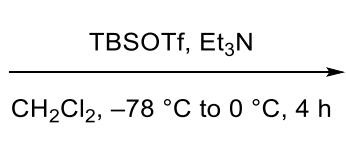

$(43 \%)$

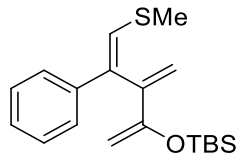

24

To a solution of diene 21 (10 mg, $50 \mu \mathrm{mol}, 1$ equiv) in dichloromethane $(0.4 \mathrm{~mL})$ under argon were subsequently added triethylamine $(24 \mu \mathrm{L}, 0.12 \mathrm{mmol}, 2.5$ equiv $)$ and tertbutyldimethylsilyl trifluoromethanesulfonate $\left(21 \mu \mathrm{L}, 0.10 \mathrm{mmol}, 2.0\right.$ equiv) at $-78{ }^{\circ} \mathrm{C}$. After one hour, the reaction mixture was allowed to warm to $0^{\circ} \mathrm{C}$. After three hours, saturated aqueous sodium bicarbonate solution $(5 \mathrm{~mL})$ and dichloromethane $(5 \mathrm{~mL})$ were added. The layers were separated, and the aqueous phase was extracted with dichloromethane $(3 \times 10 \mathrm{~mL})$. The combined organic layers were washed with saturated aqueous sodium chloride solution $(20 \mathrm{~mL})$, dried over sodium sulfate, filtered and concentrated. The crude product was purified by flash-column chromatography on silica gel (5\% diethyl ether in pentane, deactivated by using $0.5 \%$ triethylamine) to furnish [3]dendralene 24 (6.5 mg, 43\%) as a colorless liquid.

TLC (10\% diethyl ether in pentane): $R_{\mathrm{f}}=0.80\left(\mathrm{UV}, \mathrm{KMnO}_{4}\right)$. 
${ }^{1} \mathrm{H}$ NMR $\left(\mathrm{CDCl}_{3}, 400 \mathrm{MHz}\right): \delta=7.38-7.34(\mathrm{~m}, 2 \mathrm{H}), 7.30-7.25(\mathrm{~m}, 2 \mathrm{H}), 7.22-7.17(\mathrm{~m}$, $1 \mathrm{H}), 6.58(\mathrm{~s}, 1 \mathrm{H}), 5.95-5.94(\mathrm{~m}, 1 \mathrm{H}), 5.19-5.17(\mathrm{~m}, 1 \mathrm{H}), 4.38-4.36(\mathrm{~m}, 1 \mathrm{H}), 4.32-4.30$ $(\mathrm{m}, 1 \mathrm{H}), 2.38(\mathrm{~s}, 3 \mathrm{H}), 1.02-0.99(\mathrm{~m}, 9 \mathrm{H}), 0.22-0.20(\mathrm{~m}, 6 \mathrm{H})$.

${ }^{13} \mathrm{C}$ NMR $\left(\mathrm{CDCl}_{3}, 101 \mathrm{MHz}\right): \delta=153.4,143.5,139.9,136.7,128.6,128.5,126.8,125.3$, 117.9, 95.8, 29.9, 26.1, 18.5, 17.7, -4.3.

IR (Diamond-ATR, $\left.\mathrm{CDCl}_{3}\right): \tilde{v}_{\max }=2956(w), 2927(m), 1581(w), 1353(m), 1254(m)$, $1155(m), 1015(s), 827(v s), 781(m), 694(w)$.

HRMS (ESI) calc. for $\mathrm{C}_{19} \mathrm{H}_{28}{ }^{107} \mathrm{AgOSSi}\left[\mathrm{M}+{ }^{107} \mathrm{Ag}\right]^{+}:$439.0676; found: 439.0672 .

\subsubsection{Quaternary Carbon Centers}

\section{Thiophenes 26 and S-48}

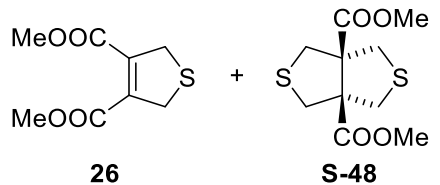

Following the General Procedures GP1 and GP2, dimethyl acetylenedicarboxylate (25, $71 \mathrm{mg}, 0.50 \mathrm{mmol}$ ) was converted into thiophenes 26 and S-48. Purification by flash-column chromatography on silica gel (20\% ethyl acetate in hexanes) furnish 26 (GP1: $60 \mathrm{mg}, 59 \%$; GP2: $78 \mathrm{mg}, 77 \%$ ) as a pale-yellow oil and S-48 (GP1: $16 \mathrm{mg}, 12 \%$; GP2: not detected) as a colorless solid. The obtained analytical data for $\mathbf{2 6}$ were in full agreement with those reported in literature. ${ }^{24}$

\section{Dihydrothiophene 26:}

TLC (30\% ethyl acetate in hexanes): $R_{\mathrm{f}}=0.54\left(\mathrm{KMnO}_{4}\right)$.

${ }^{1} \mathrm{H}$ NMR $\left(\mathrm{CDCl}_{3}, 400 \mathrm{MHz}\right): \delta=4.05(\mathrm{~s}, 4 \mathrm{H}), 3.80(\mathrm{~s}, 6 \mathrm{H})$.

${ }^{13} \mathrm{C}$ NMR $\left(\mathrm{CDCl}_{3}, 101 \mathrm{MHz}\right): \delta=164.7,138.6,52.7,39.5$.

IR (Diamond-ATR, $\left.\mathrm{CDCl}_{3}\right): \tilde{v}_{\max }=2953(w), 1724(v s), 1659(w), 1435(m), 1319(m)$, $1277(s), 1213(s), 1081(m), 759(w)$.

HRMS (El) calc. for $\mathrm{C}_{8} \mathrm{H}_{10} \mathrm{O}_{4} \mathrm{~S}$ [M]+: 202.0294; found: 202.0289. 


\section{Tetrahydrothiophene S-48:}

TLC $(20 \%$ ethyl acetate in hexanes $): R_{\mathrm{f}}=0.32\left(\mathrm{KMnO}_{4}\right)$.

${ }^{1} \mathrm{H}$ NMR $\left(\mathrm{CDCl}_{3}, 400 \mathrm{MHz}\right): \delta=3.75(\mathrm{~s}, 6 \mathrm{H}), 3.47(\mathrm{~d}, J=11.5 \mathrm{~Hz}, 4 \mathrm{H}), 3.23(\mathrm{~d}, J=11.4 \mathrm{~Hz}$, $4 \mathrm{H})$.

${ }^{13} \mathrm{C}$ NMR $\left(\mathrm{CDCl}_{3}, 101 \mathrm{MHz}\right): \delta=172.7,67.6,52.8,38.6$.

IR (Diamond-ATR, $\left.\mathrm{CDCl}_{3}\right): \tilde{v}_{\max }=2959(w), 1723(v s), 1438(m), 1300(s), 1242(s)$, $1200(m), 1151(m), 1053(m), 1018(w), 1003(w)$.

HRMS (El) calc. for $\mathrm{C}_{10} \mathrm{H}_{14} \mathrm{O}_{4} \mathrm{~S}_{2}[\mathrm{M}]^{+}:$262.0328; found: 262.0321.

$\mathrm{MP}=104-106^{\circ} \mathrm{C}$

\section{Tetrahydrothiophene 27}
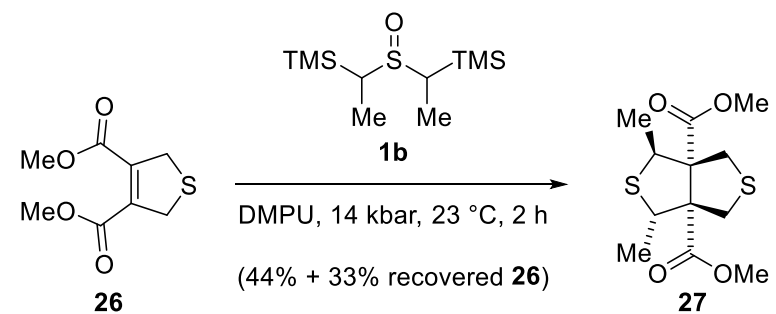

Following the General Procedures GP1 (14 kbar) and GP2, dihydrothiophene 26 (40 mg, $0.20 \mathrm{mmol}$ ) was converted into tetrahydrothiophene 27 by using sulfoxide $\mathbf{1 b}(0.20 \mathrm{~g}$, $0.79 \mathrm{mmol}, 4.0$ equiv). Purification by flash-column chromatography on silica gel (10\% diethyl ether in pentane) furnish 27 (GP1: $25 \mathrm{mg}, 44 \%$; GP2: not detected) as a colorless oil and remaining starting material 26 (GP1: $13 \mathrm{mg}, 33 \%$ ).

TLC (10\% diethyl ether in pentane $): R_{\mathrm{f}}=0.38\left(\mathrm{KMnO}_{4}, \mathrm{CAM}\right)$

${ }^{1} \mathbf{H}$ NMR $\left(\mathrm{CDCl}_{3}, 400 \mathrm{MHz}\right): \delta=4.44(\mathrm{q}, J=6.8 \mathrm{~Hz}, 1 \mathrm{H}), 3.77(\mathrm{q}, J=6.8 \mathrm{~Hz}, 1 \mathrm{H}), 3.75(\mathrm{~s}$, $3 \mathrm{H}), 3.74(\mathrm{~s}, 3 \mathrm{H}), 3.62(\mathrm{~d}, J=11.5 \mathrm{~Hz}, 1 \mathrm{H}), 3.53(\mathrm{~d}, J=11.8 \mathrm{~Hz}, 1 \mathrm{H}), 3.21(\mathrm{~d}, J=11.7 \mathrm{~Hz}$, $1 \mathrm{H}), 2.99(\mathrm{~d}, J=11.5 \mathrm{~Hz}, 1 \mathrm{H}), 1.41(\mathrm{~d}, J=6.8 \mathrm{~Hz}, 3 \mathrm{H}), 1.17(\mathrm{~d}, J=6.8 \mathrm{~Hz}, 3 \mathrm{H})$.

${ }^{13} \mathrm{C}$ NMR $\left(\mathrm{CDCl}_{3}, 101 \mathrm{MHz}\right): \delta=172.5,171.1,72.1,71.7,52.9,52.1,46.1,45.3,36.2,35.1$, 17.3, 16.2 .

IR (Diamond-ATR, $\mathrm{CDCl}_{3}$ ): $\tilde{v}_{\max }=2951(w), 1731(v s), 1446(w), 1378(w), 1344(w)$, $1280(m), 1217(m), 1141(w), 834(w)$. 
HRMS (ESI) calc. for $\mathrm{C}_{12} \mathrm{H}_{18} \mathrm{NaO}_{4} \mathrm{~S}_{2}[\mathrm{M}+\mathrm{Na}]^{+}: 313.0539$; found: 313.0531 .

\section{Diol meso-28}

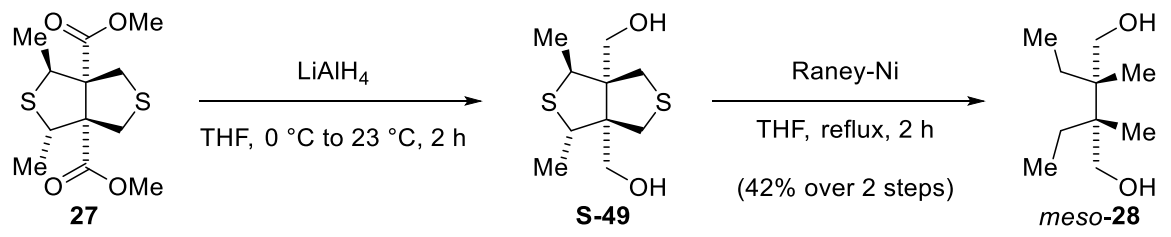

To a solution of tetrahydrothiophene 27 (12 mg, $41 \mu \mathrm{mol}, 1$ equiv) in tetrahydrofuran $(2.0 \mathrm{~mL})$ under argon was added lithium aluminum hydride $(6.2 \mathrm{mg}, 0.16 \mathrm{mmol}, 4.0$ equiv) in one portion at $0{ }^{\circ} \mathrm{C}$. Upon addition, the reaction mixture was allowed to warm to $23^{\circ} \mathrm{C}$. After two hours, saturated aqueous potassium sodium tartrate solution (Rochelle's salt, $2 \mathrm{~mL}$ ) and dichloromethane $(5 \mathrm{~mL})$ were added. After one hour, the layers were separated, and the aqueous layer was extracted with dichloromethane $(3 \times 10 \mathrm{~mL})$. The combined organic layers were dried over sodium sulfate, filtered and concentrated. The crude diol S-49 was used in the next step without further purification.

TLC (5\% methanol in dichloromethane): $R_{\mathrm{f}}=0.42\left(\mathrm{KMnO}_{4}, \mathrm{CAM}\right)$.

HRMS (ESI) calc. for $\mathrm{C}_{10} \mathrm{H}_{18} \mathrm{NaO}_{2} \mathrm{~S}_{2}[\mathrm{M}+\mathrm{Na}]^{+}: 257.0640$; found: 257.0638 .

To a suspension of Raney ${ }^{\circledR}$-Nickel (0.25 g, W.R. Grace and Co. Raney ${ }^{\circledR} 2800$, slurry in water) in tetrahydrofuran $(0.25 \mathrm{~mL})$ was added a solution of crude diol S-49 in tetrahydrofuran $(0.25 \mathrm{~mL})$ at $23^{\circ} \mathrm{C}$. Upon addition, the reaction mixture was heated to $70{ }^{\circ} \mathrm{C}$. After two hours, the suspension was filtered through a plug of Celite ${ }^{\circledR}$ and the filtrate was concentrated. The crude product was purified by flash-column chromatography on silica gel (5\% methanol in dichloromethane) to furnish meso-28 (3.0 mg, 42\% over 2 steps) as a colorless oil.

TLC (10\% methanol in dichloromethane): $R_{\mathrm{f}}=0.47(\mathrm{CAM})$.

${ }^{1} \mathbf{H}$ NMR $\left(\mathrm{CDCl}_{3}, 400 \mathrm{MHz}\right): \delta=3.51(\mathrm{~s}, 4 \mathrm{H}), 3.30(\mathrm{~s}, 2 \mathrm{H}), 1.52(\mathrm{dt}, J=14.7,7.3 \mathrm{~Hz}, 2 \mathrm{H})$, $1.35-1.27(\mathrm{~m}, 2 \mathrm{H}), 0.85(\mathrm{t}, J=7.5 \mathrm{~Hz}, 6 \mathrm{H}), 0.77(\mathrm{~d}, J=0.5 \mathrm{~Hz}, 6 \mathrm{H})$.

${ }^{13} \mathrm{C}$ NMR $\left(\mathrm{CDCl}_{3}, 101 \mathrm{MHz}\right): \delta=65.4,42.6,24.4,16.8,9.0$.

IR (Diamond-ATR, $\left.\mathrm{CDCl}_{3}\right)$ : $\tilde{v}_{\max }=3202(b r), 2966(s), 2926(v s), 2882(s), 1671(w)$, $1464(m), 1381(w), 1068(m), 1038(m), 1003(w)$.

HRMS (ESI) calc. for $\mathrm{C}_{10} \mathrm{H}_{22} \mathrm{NaO}_{2}[\mathrm{M}+\mathrm{Na}]^{+}:$197.1512; found: 197.1511 . 
Note: Treatment of diol S-49 with Raney ${ }^{\circledR}$-Nickel in tetrahydrofuran at ambient temperature $\left(23^{\circ} \mathrm{C}\right)$ exclusively led to the cleavage of the less-substituted tetrahydrothiophene ring.

\subsubsection{NGB 4420}

\section{Tetrahydrothiophene 30}

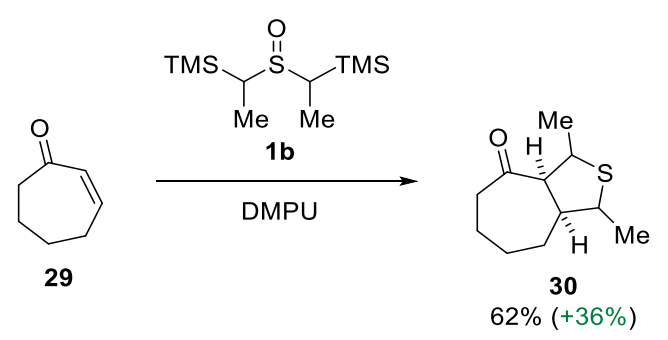

Following the General Procedures GP1 (14 kbar) and GP2, 2-Cyclohepten-1-one (29, 55 mg, $0.50 \mathrm{mmol}$ ) was converted into tetrahydrothiophene 30 by using sulfoxide $\mathbf{1 b}(0.25 \mathrm{~g}$, $1.0 \mathrm{mmol}$, 2.0 equiv). Purification by flash-column chromatography on silica gel (dichloromethane) furnished 30 (GP1: $62 \mathrm{mg}, 62 \%$; GP2: $26 \mathrm{mg}, 26 \%$; inseparable mixture of inconsequential diastereomers) as a colorless oil.

TLC (dichloromethane): $R_{\mathrm{f}}=0.28\left(\mathrm{KMnO}_{4}\right)$.

HRMS (ESI) calc. for $\mathrm{C}_{11} \mathrm{H}_{18}{ }^{107} \mathrm{AgOS}\left[\mathrm{M}+{ }^{107} \mathrm{Ag}\right]^{+}: 305.0124$; found: 305.0119 .

Note: Separation of the diastereomers of tetrahydrothiophene $\mathbf{3 0}$ was unsuccessful. ${ }^{1} \mathrm{H}$ and ${ }^{13} \mathrm{C}$ NMR data of the mixture is displayed in section 4 , however the assignment to determine the diastereomeric ratio was unproductive.

\section{Thiophene 31}

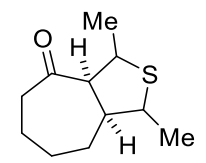

30

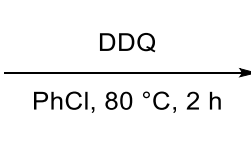

$(60 \%)$

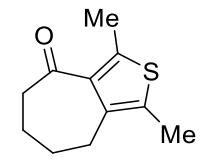

31

To a solution of tetrahydrothiophene $30(20 \mathrm{mg}, 0.10 \mathrm{mmol}, 1$ equiv $)$ in chlorobenzene $(20 \mathrm{~mL})$ under argon was added 2,3-dichloro-5,6-dicyano-1,4-benzochinone (57 mg, $0.25 \mathrm{mmol}, 2.5$ equiv) in one portion at $23^{\circ} \mathrm{C}$. Upon addition, the reaction mixture was heated to $80^{\circ} \mathrm{C}$. After two hours, the brownish suspension was filtered and concentrated. The crude product was purified by flash-column chromatography on silica gel (10\% diethyl ether in pentane) to furnish thiophene $\mathbf{3 1}(12 \mathrm{mg}, 60 \%)$ as a yellow oil. 
TLC (dichloromethane): $R_{\mathrm{f}}=0.26\left(\mathrm{UV}, \mathrm{CAM}, \mathrm{KMnO}_{4}\right)$.

${ }^{1} \mathrm{H}$ NMR $\left(\mathrm{CDCl}_{3}, 600 \mathrm{MHz}\right): \delta=2.73-2.69(\mathrm{~m}, 2 \mathrm{H}), 2.67-2.62(\mathrm{~m}, 2 \mathrm{H}), 2.55(\mathrm{~s}, 3 \mathrm{H}), 2.29$ (s, 3H), $1.83-1.75(\mathrm{~m}, 4 \mathrm{H})$.

${ }^{13} \mathrm{C} \mathrm{NMR}\left(\mathrm{CDCl}_{3}, 201 \mathrm{MHz}\right): \delta=200.8,143.9,138.2,136.8,128.4,41.5,25.1,25.1,22.0$, $15.4,12.8$.

IR (Diamond-ATR, $\mathrm{CDCl}_{3}$ ): $\tilde{v}_{\max }=2925(s), 2861(w), 1665(v s), 1553(w), 1477(m)$, $1455(m), 1375(w), 1261(m), 1131(w), 845(w)$.

HRMS (ESI) calc. for $\mathrm{C}_{11} \mathrm{H}_{15} \mathrm{OS}[\mathrm{M}+\mathrm{H}]^{+}:$195.0838; found: 195.0838 .

\subsubsection{Tenilapine}

\section{Dienone 33}

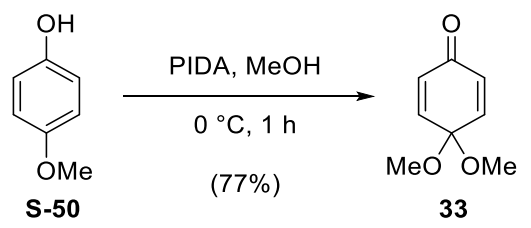

To a solution of 4-methoxyphenol S-50 (1.0 g, $8.1 \mathrm{mmol}, 1$ equiv) in methanol ( $20 \mathrm{~mL})$ under argon was slowly added (diacetoxyiodo)benzene $\left(2.6 \mathrm{~g}, 8.1 \mathrm{mmol}, 1.0\right.$ equiv) at $0{ }^{\circ} \mathrm{C}$. After one hour, saturated aqueous sodium bicarbonate solution $(30 \mathrm{~mL})$ was added and the aqueous layer was extracted with diethyl ether $(3 \times 30 \mathrm{~mL})$. The combined organic layers were washed with saturated aqueous sodium chloride solution $(50 \mathrm{~mL})$, dried over magnesium sulfate, filtered and concentrated. The crude product was purified by flashcolumn chromatography on silica gel ( $20 \%$ ethyl acetate in cyclohexane, deactivated by using $0.5 \%$ triethylamine) to furnish dienone $33(0.96 \mathrm{~g}, 77 \%)$ as a yellow oil. The obtained analytical data were in full agreement with those reported in literature. ${ }^{25}$

$\operatorname{TLC}\left(20 \%\right.$ ethyl acetate in cyclohexane): $\mathrm{R}_{f}=0.30\left(\mathrm{UV}, \mathrm{KMnO}_{4}, \mathrm{CAM}\right)$. 


\section{Tetrahydrothiophenes $34 a$, cis-34b and trans-34b}

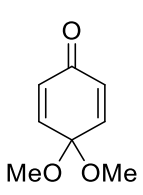

33

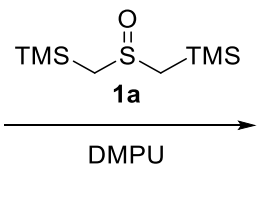

$$
\begin{gathered}
34 \mathbf{a} \\
49 \%(+7 \%)
\end{gathered}
$$
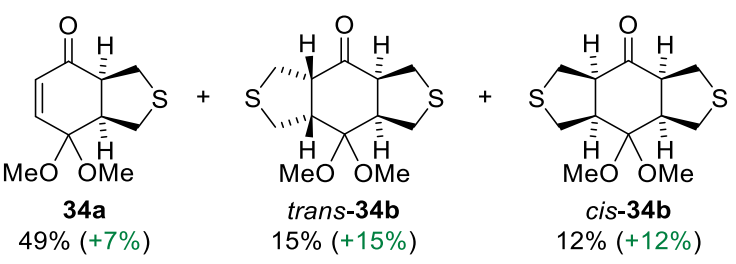

Following General Procedures GP1 (14 kbar) and GP2, dienone 33 (77 mg, 0.50 mmol) was converted into tetrahydrothiophenes $\mathbf{3 4 a}$, trans-34b and cis-34b by using sulfoxide $\mathbf{1 a}$ $(2+2$ equiv., see Note). Purification by flash-column chromatography on silica gel $(10 \%$ to 50\% ethyl acetate in cyclohexane) furnished 34a (GP1: $104 \mathrm{mg}, 49 \%$; GP2: $90 \mathrm{mg}, 42 \%$ ) as a colorless, crystalline solid, trans-34b (GP1: 42 mg, 15\%; GP2: not detected) as a colorless oil, cis-34b (HP: 33 mg, 12\%; GP2: not detected) as a colorless solid.

\section{Monoadduct 34a:}

TLC (25\% diethyl ether in pentane): $R_{\mathrm{f}}=0.40\left(\mathrm{UV}, \mathrm{KMnO}_{4}\right)$.

${ }^{1} \mathrm{H}$ NMR $\left(\mathrm{CDCl}_{3}, 400 \mathrm{MHz}\right): \delta=6.70(\mathrm{dt}, J=10.4,1.9 \mathrm{~Hz}, 1 \mathrm{H}), 6.06(\mathrm{dd}, J=10.4,1.7 \mathrm{~Hz}$, $1 \mathrm{H}), 3.57-3.49(\mathrm{~m}, 1 \mathrm{H}), 3.33(\mathrm{~d}, J=1.5 \mathrm{~Hz}, 3 \mathrm{H}), 3.30(\mathrm{~d}, J=1.5 \mathrm{~Hz}, 3 \mathrm{H}), 3.26(\mathrm{t}, J=$ $5.8 \mathrm{~Hz}, 1 \mathrm{H}$ ), 3.06 (dddd, $J=11.2,7.8,5.5,2.3 \mathrm{~Hz}, 1 \mathrm{H}), 2.95-2.86(\mathrm{~m}, 2 \mathrm{H}), 2.63$ (td, $J=$ $11.1,1.8 \mathrm{~Hz}, 1 \mathrm{H})$.

${ }^{13} \mathrm{C}$ NMR $\left(\mathrm{CDCl}_{3}, 101 \mathrm{MHz}\right): \delta=197.2,146.0,129.5,98.3,52.0,50.0,49.9,48.3,31.9,30.2$.

IR (Diamond-ATR, $\left.\mathrm{CDCl}_{3}\right): \tilde{v}_{\max }=2941(\mathrm{~m}), 1683(\mathrm{~s}), 1455(w), 1382(\mathrm{~m}), 1259(w)$, $1223(w), 1096(v s), 1049(v s), 963(m), 952(m)$.

HRMS (ESI) calc. for $\mathrm{C}_{10} \mathrm{H}_{14} \mathrm{NaO}_{3} \mathrm{~S}$ [M+Na] $]^{+}:$237.0556; found: 237.0549 .

MP $=97-99^{\circ} \mathrm{C}$

Double adduct trans-34b:

TLC (25\% diethyl ether in pentane): $R_{\mathrm{f}}=0.37\left(\mathrm{KMnO}_{4}\right)$.

${ }^{1} \mathrm{H}$ NMR $\left(\mathrm{CDCl}_{3}, 400 \mathrm{MHz}\right): \delta=3.30(\mathrm{~s}, 6 \mathrm{H}), 3.22-3.03(\mathrm{~m}, 10 \mathrm{H}), 2.98(\mathrm{dd}, J=11.3,7.0 \mathrm{~Hz}$, $2 \mathrm{H})$.

${ }^{13} \mathrm{C}$ NMR $\left(\mathrm{CDCl}_{3}, 101 \mathrm{MHz}\right): \delta=209.3,100.7,53.5,50.0,49.7,33.6,33.1$.

IR (Diamond-ATR, $\left.\mathrm{CDCl}_{3}\right): \tilde{v}_{\max }=2941(m), 2832(w), 1712(s), 1462(w), 1340(w)$, $1220(w), 1125(m), 1050(s), 974(w), 852(w)$. 
HRMS (ESI) calc. for $\mathrm{C}_{12} \mathrm{H}_{18} \mathrm{NaO}_{3} \mathrm{~S}_{2}[\mathrm{M}+\mathrm{Na}]^{+}:$297.0590; found: 297.0581 .

$M P=80-82^{\circ} \mathrm{C}$.

Double adduct cis-34b:

TLC (25\% diethyl ether in pentane): $R_{\mathrm{f}}=0.29\left(\mathrm{KMnO}_{4}\right)$.

${ }^{1} \mathrm{H}$ NMR $\left(\mathrm{CDCl}_{3}, 400 \mathrm{MHz}\right): \delta=3.64(\mathrm{dd}, J=10.9,1.1 \mathrm{~Hz}, 2 \mathrm{H}), 3.36(\mathrm{~s}, 3 \mathrm{H}), 3.32-3.27(\mathrm{~m}$, 5H), 3.14 (dddd, $J=10.0,8.9,7.5,2.3 \mathrm{~Hz}, 2 \mathrm{H}$ ), 2.89 (dd, $J=11.3,7.5 \mathrm{~Hz}, 2 \mathrm{H}$ ), $2.80-2.71$ $(\mathrm{m}, 4 \mathrm{H})$.

${ }^{13} \mathrm{C}$ NMR $\left(\mathrm{CDCl}_{3}, 101 \mathrm{MHz}\right): \delta=204.4,100.6,52.5,49.0,48.4,30.9,29.0$.

IR (Diamond-ATR, $\left.\mathrm{CDCl}_{3}\right): \tilde{v}_{\max }=2941(m), 1718(s), 1459(w), 1371(m), 1269(w)$, $1176(w), 1107(s), 1050(s), 984(m), 936(m)$.

HRMS (ESI) calc. for $\mathrm{C}_{12} \mathrm{H}_{18} \mathrm{NaO}_{3} \mathrm{~S}_{2}[\mathrm{M}+\mathrm{Na}]^{+}:$297.0590; found: 297.0581 .

Note: GP1 and GP2 were modified in terms of repeated treatment with sulfoxide 1a (2 equiv) and elongated reaction times (GP1: additional two hours; GP2: additional one hour).

\section{Thiophene 35}

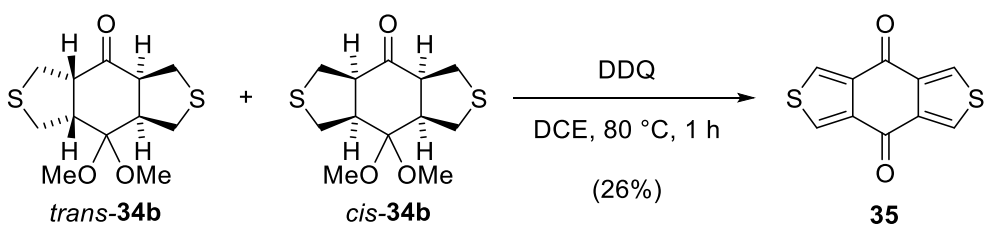

To a solution of a mixture of tetrahydrothiophenes trans-35b and cis-35b $(20 \mathrm{mg}, 73 \mu \mathrm{mol}$, 1 equiv) in 1,2-dichloroethane (1 mL) was added 2,3-dichloro-5,6-dicyano-1,4-benzochinone (84 mg, $0.36 \mathrm{mmol}, 5.0$ equiv). Upon addition, the reaction mixture was heated to $80^{\circ} \mathrm{C}$. After one hour, the reaction was allowed to cool down to $23^{\circ} \mathrm{C}$, filtered through a plug of Celite $^{\circledR}$ and the filtrate was concentrated. The residue was purified by flash-column chromatography on silica gel (cyclohexane/toluene/ethyl acetate $=6: 3: 1$ ) to yield thiophene $\mathbf{3 6}$ as a yellow solid (4.2 $\mathrm{mg}, 26 \%)$.

TLC (cyclohexane/toluene/ethyl acetate $=6: 3: 1): R_{f}=0.15\left(\mathrm{UV}, \mathrm{KMnO}_{4}\right)$.

${ }^{1} \mathrm{H}$ NMR $\left(\mathrm{CD}_{2} \mathrm{Cl}_{2} / \mathrm{d}-\mathrm{TFA}=3: 1,300 \mathrm{MHz}\right): \delta=8.58(\mathrm{~s}, 4 \mathrm{H})$.

${ }^{13} \mathrm{C}$ NMR $\left(\mathrm{CD}_{2} \mathrm{Cl}_{2} / \mathrm{d}-\mathrm{TFA}=3: 1,75 \mathrm{MHz}\right): \delta=178.7,139.1,137.5$. 
IR (Diamond-ATR, $\mathrm{CDCl}_{3}$ ): $\tilde{v}_{\max }=2921(\mathrm{~s}), 2852(\mathrm{~m}), 1651(\mathrm{~m}), 1504(\mathrm{~m}), 1448(\mathrm{~s}), 1376$ $(w), 1229(s), 861(m), 845(m), 729(m)$.

HRMS (ESI) calc. for $\mathrm{C}_{10} \mathrm{H}_{5} \mathrm{O}_{2} \mathrm{~S}_{2}[\mathrm{M}+\mathrm{H}]^{+}:$220.9725; found: 220.9715 .

MP $=162^{\circ} \mathrm{C}$ (decomposition) 


\section{References}

(1) Still, W. C.; Kahn, M.; Mitra, A. Rapid chromatographic technique for preparative separations with moderate resolution. J. Org. Chem. 1978, 43, 2923-2925.

(2) Kofron, W. G.; Baclawski, L. M. A convenient method for estimation of alkyllithium concentrations. J. Org. Chem. 1976, 41, 1879-1880.

(3) Terao, Y.; Aono, M.; Imai, N.; Achiwa, K. Thiocarbonyl Ylides. VI.1) New Generation of Thiocarbonyl Ylides from Organosilicon Compounds Containing Sulfur and Their 1,3Cycloadditions. Chem. Pharm. Bull. 1987, 35, 1734-1740.

(4) Winter, N.; Trauner, D. Thiocarbonyl Ylide Chemistry Enables a Concise Synthesis of (士)-Hippolachnin A. J. Am. Chem. Soc. 2017, 139, 11706-11709.

(5) Matsuo, K.; Shindo, M. Cu(II)-Catalyzed Acylation by Thiol Esters Under Neutral Conditions: Tandem Acylation-Wittig Reaction Leading to a One-Pot Synthesis of Butenolides. Org. Lett. 2010, 12, 5346-5349.

(6) Rüedi, G.; Hansen, H.-J. Diradical-Promoted Two-Carbon Ring-Expansion Reactions by Thermal Isomerization: Synthesis of Functionalized Macrocyclic Ketones. Helvetica Chim. Acta. 2004, 87, 1628-1665.

(7) Stephens, T. C.; Lodi, M.; Steer, A. M.; Lin, Y.; Gill, M. T.; Unsworth, W. P. Synthesis of Cyclic Peptide Mimetics by the Successive Ring Expansion of Lactams. Chem. Eur. J. 2017, 23, 13314-13318.

(8) Wyler, B.; Brucelle, F.; Renaud, P. Preparation of the Core Structure of Aspidosperma and Strychnos Alkaloids from Aryl Azides by a Cascade Radical Cyclization. Org. Lett. 2016, 18, 1370-1373.

(9) Wu, H.; Garcia, J. M.; Haeffner, F.; Radomkit, S.; Zhugralin, A. R.; Hoveyda, A. H. Mechanism of NHC-Catalyzed Conjugate Additions of Diboron and Borosilane Reagents to a, $\beta$-Unsaturated Carbonyl Compounds. J. Am. Chem. Soc. 2015, 137, 10585-10602.

(10) Ciszek, B.; Fleischer, I. Homogeneous Palladium-Catalyzed Transfer Hydrogenolysis of Benzylic Alcohols Using Formic Acid as Reductant. Chem. Eur. J. 2018, 24, 1225912263.

(11) Wolstenhulme, J. R.; Rosenqvist, J.; Lozano, O.; llupeju, J.; Wurz, N.; Engle, K. M.; Pidgeon, G. W.; Moore, P. R.; Sandford, G.; Gouverneur, V. Asymmetric Electrophilic Fluorocyclization with Carbon Nucleophiles. Angew. Chem. Int. Ed. 2013, 52, 9796-9800.

(12) Qin, T.; Cornella, J.; Li, C.; Malins, L. R.; Edwards, J. T.; Kawamura, S.; Maxwell, B. D.; Eastgate, M. D.; Baran, P. S. A general alkyl-alkyl cross-coupling enabled by redox-active esters and alkylzinc reagents. Science 2016, 352, 801-805.

(13) Sirisoma, N. S.; Höld, K. M.; Casida, J. E. $\alpha$ - and $\beta$-Thujones (Herbal Medicines and Food Additives): Synthesis and Analysis of Hydroxy and Dehydro Metabolites. J. Agric. Food Chem. 2001, 49, 1915-1921.

(14) Vuagnoux-d' Augustin, M.; Alexakis, A. Influence of the Double-Bond Geometry of the Michael Acceptor on Copper-Catalyzed Asymmetric Conjugate Addition. Eur. J. Org. Chem. 2007, 5852-5860. 
(15) Konradova, D.; Kozubikova, H.; Dolezal, K.; Pospisil, J. Microwave-Assisted Synthesis of Phenylpropanoids and Coumarins: Total Synthesis of Osthol. Eur. J. Org. Chem. 2017, 5204-5213.

(16) Peng, Y.; Li, J.; Sun, Y.; Chan, J. Y.-W.; Sheng, D.; Wang, K.; Wei, P.; Ouyang, P.; Wang, D.; Lee, S. M. Y.; Zhou, G.-C. SAR studies of 3,14,19-derivatives of andrographolide on anti-proliferative activity to cancer cells and toxicity to zebrafish: an in vitro and in vivo study. RSC Adv. 2015, 5, 22510-22526.

(17) Alunni, S.; Laureti, V.; Ottavi, L.; Ruzziconi, R. Catalysis of the $\beta$-Elimination of HF from Isomeric 2-Fluoroethylpyridines and 1-Methyl-2-fluoroethylpyridinium Salts. ProtonActivating Factors and Methyl-Activating Factors as a Mechanistic Test To Distinguish between Concerted E2 and E1cb Irreversible Mechanisms. J. Org. Chem. 2003, 68, 718725 .

(18) Shiramizu, M.; Toste, F. D. Deoxygenation of Biomass-Derived Feedstocks: Oxorhenium-Catalyzed Deoxydehydration of Sugars and Sugar Alcohols. Angew. Chem. Int. Ed. 2012, 51, 8082-8086.

(19) Yildizan, S.; Schulz, S. Prolinamide/PPTS-Catalyzed Hajos-Parrish Annulation: Efficient Approach to the Tricyclic Core of Cylindricine-Type Alkaloids. Synlett 2011, 19, 2831-2833.

(20) Sadamitsu, Y.; Komatsuki, K.; Saito, K.; Yamada, T. Access to Tetronic Acids via Silver-Catalyzed CO2 Incorporation into Conjugated Ynones. Org. Lett. 2017, 19, 31913194.

(21) Yoon, J.; Ryu, J.-S. A rapid synthesis of lavendustin-mimetic small molecules by click fragment assembly. Bioorg. Med. Chem. Lett. 2010, 20, 3930-3935.

(22) Ye, X.-S.; Wong, H. N. C. Synthetic Applications of 3,4-Bis(trimethylsilyl)thiophene: Unsymmetrically 3,4-Disubstituted Thiophenes and 3,4-Didehydrothiophene. J. Org. Chem. 1997, 62, 1940-1954.

(23) Flemming, I.; Lewis, J. J. The diastereoselectivity of electrophilic attack on trigonal carbon adjacent to a stereogenic centre: diastereoselective alkylation and protonation of open-chain enolates having a stereogenic centre at the $\beta$ position. J. Chem. Soc., Perkin Trans. 11992, 24, 3357-3366.

(24) Aono, M.; Hyodo, C.; Terao, Y.; Achiwa, K. Generation of thiocarbonyl ylides with release of disiloxane from bis(trimethylsilylmethyl) sulfoxides. Tetrahedron Lett. 1986, 27, 4039-4042.

(25) Yi, C.-B.; She, Z.-Y.; Cheng, Y.-F.; Qu, J. Redox-Neutral $\alpha-C-H$ Functionalization of Pyrrolidin-3-ol. Org. Lett. 2018, 20, 668-671. 


\section{$4 \quad{ }^{1} \mathrm{H}$ and ${ }^{13} \mathrm{C}$ NMR Spectra}

TMS S $\sim$ TMS

S-2

${ }^{1} \mathrm{H} \mathrm{NMR}\left(\mathrm{CDCl}_{3}, 400 \mathrm{MHz}\right)$
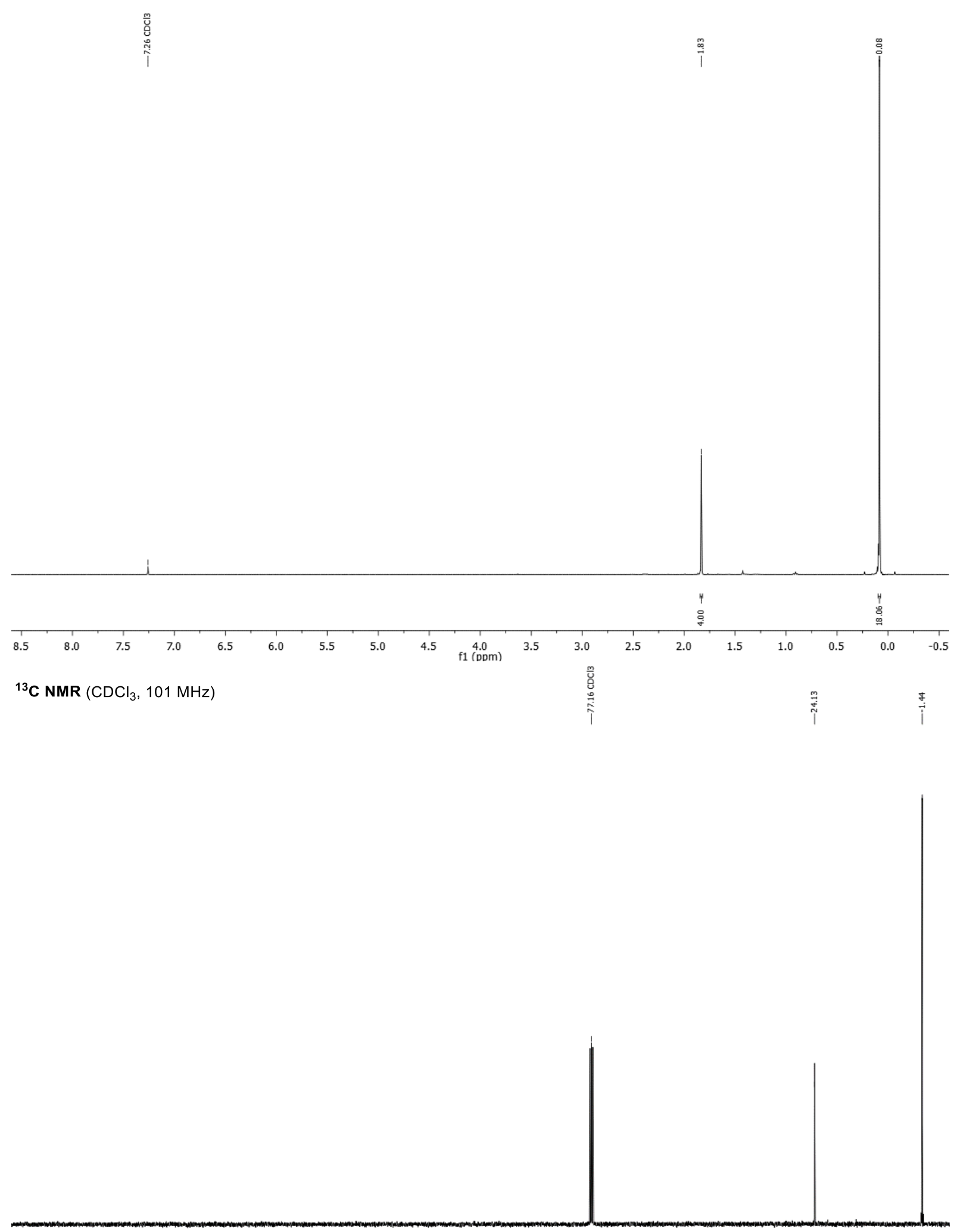

$\begin{array}{lllllllllll}210 & 200 & 190 & 180 & 170 & 160 & 150 & 140 & 130 & 120 & 110 \\ \mathrm{f} 1(\mathrm{ppm}) & 100\end{array}$ 
TMS $\stackrel{\text { II }}{\text { STMS }}$

$1 \mathrm{a}$

${ }^{1} \mathrm{H} \mathrm{NMR}\left(\mathrm{CDCl}_{3}, 400 \mathrm{MHz}\right)$

$\underset{\substack{1 \\ 0}}{\substack{0 \\ 0}}$

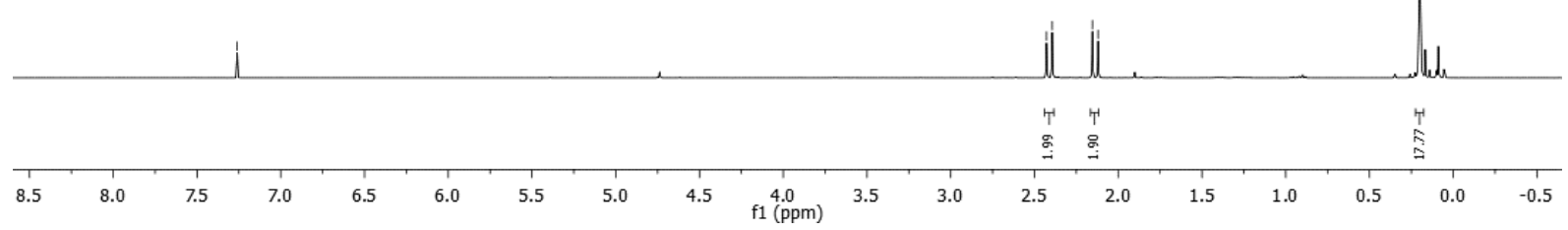

${ }^{13} \mathrm{C} \mathrm{NMR}\left(\mathrm{CDCl}_{3}, 101 \mathrm{MHz}\right)$
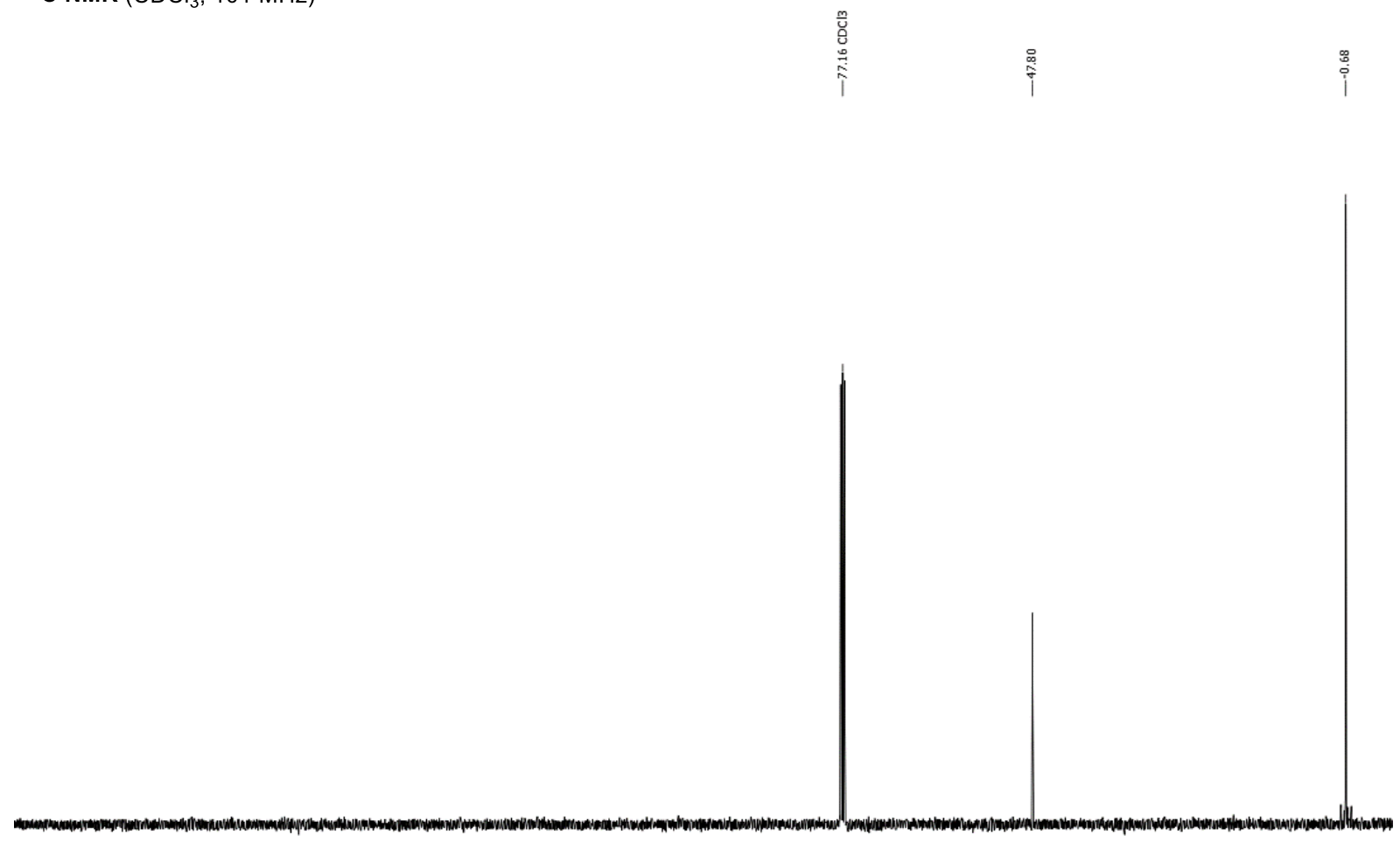

200 190180 $\begin{array}{lllllll}170 & 160 & 150 & 140 & 130 & 120 & 110\end{array}$ 100
$f 1(\mathrm{ppm})$ 
TMS S $\rightleftharpoons$ OTMS

S-5

${ }^{1} \mathrm{H}$ NMR $\left(\mathrm{CD}_{2} \mathrm{Cl}_{2}, 400 \mathrm{MHz}\right)$

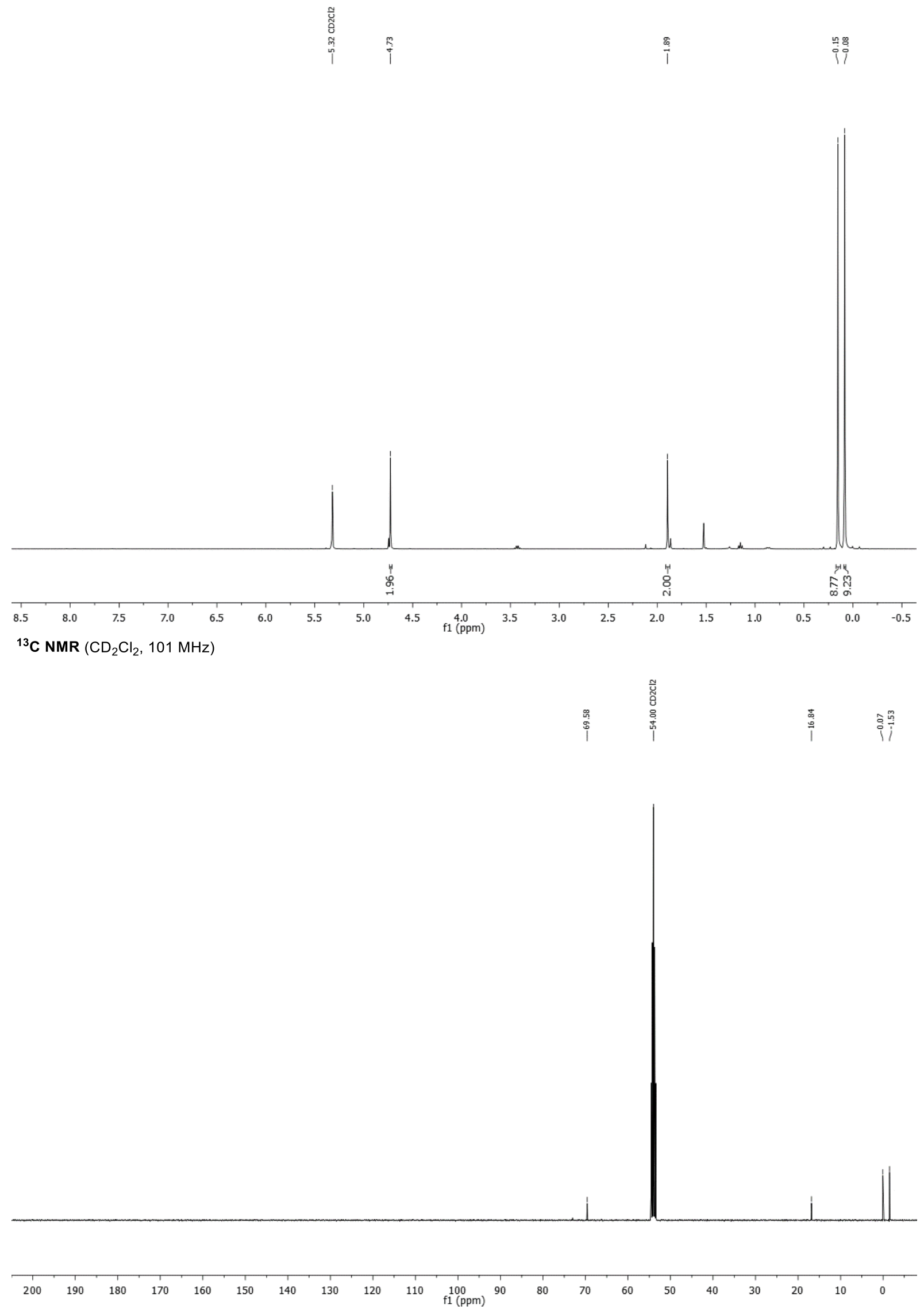




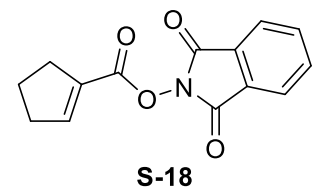

${ }^{1} \mathrm{H}$ NMR $\left(\mathrm{CDCl}_{3}, 600 \mathrm{MHz}\right)$

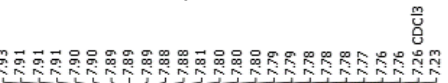

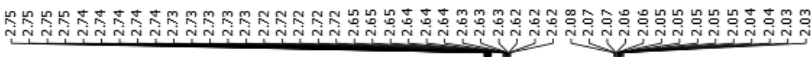

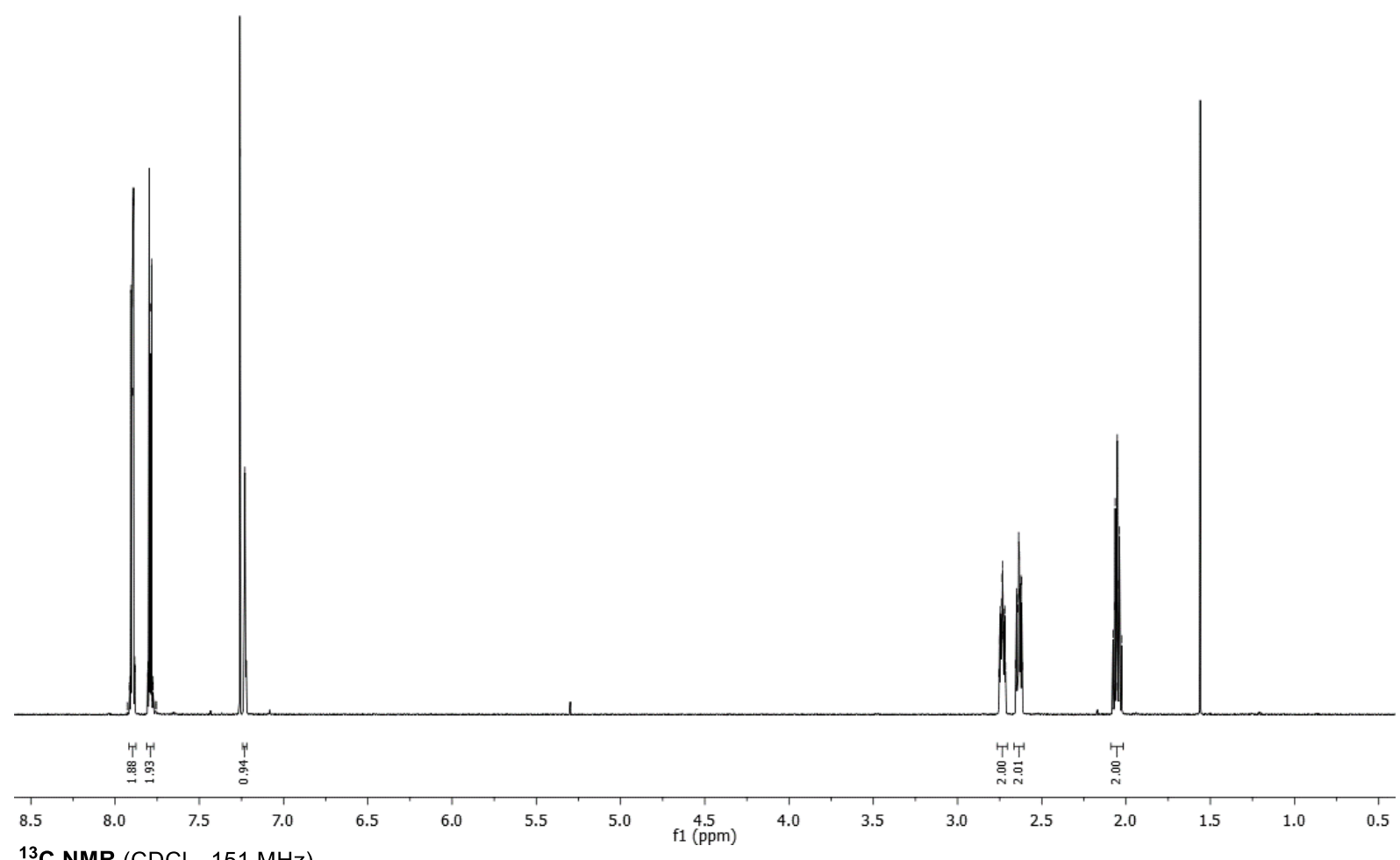

${ }^{13} \mathrm{C}$ NMR $\left(\mathrm{CDCl}_{3}, 151 \mathrm{MHz}\right)$

آ

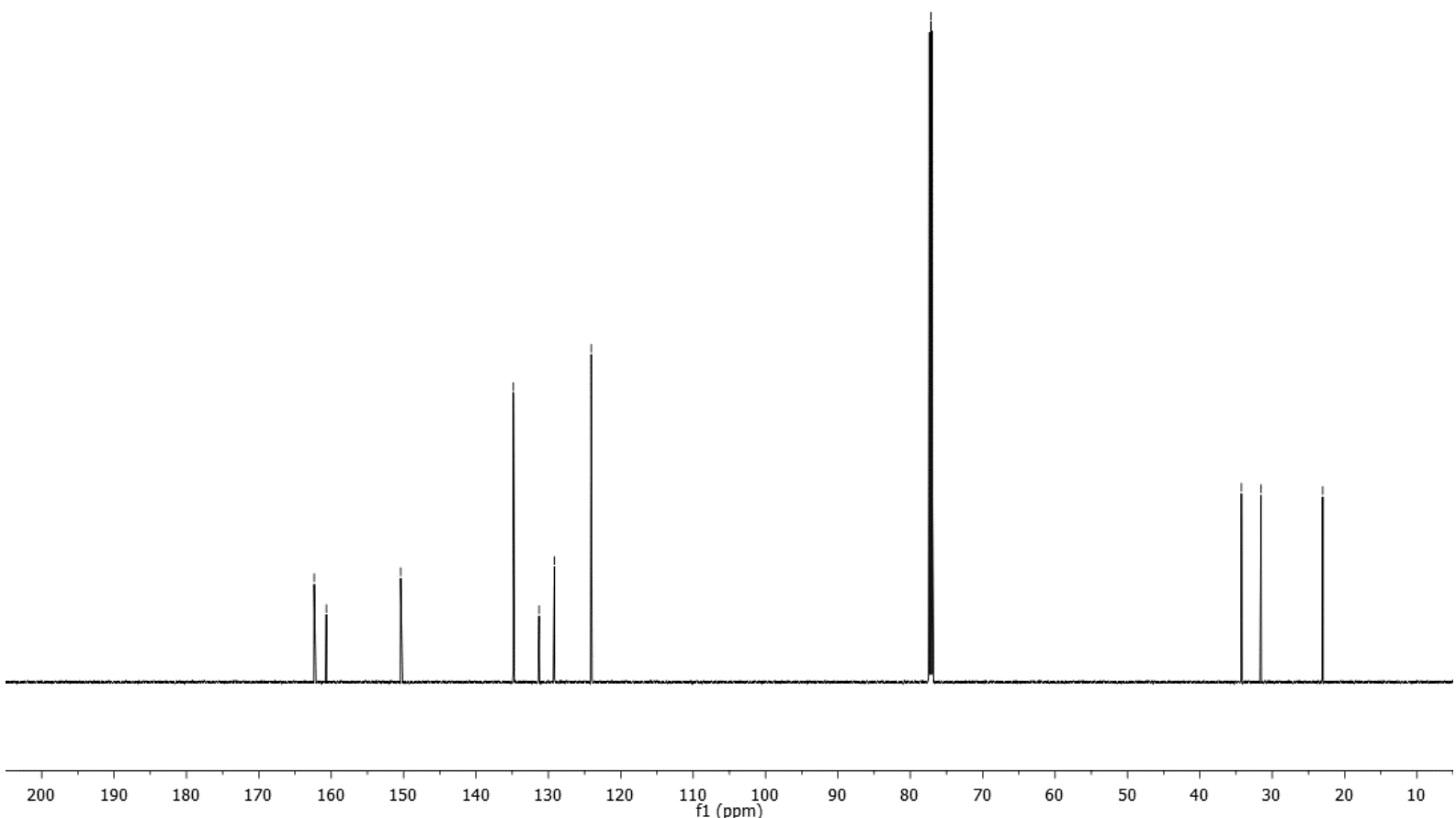




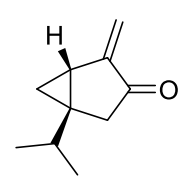

S-21

${ }^{1} \mathrm{H}$ NMR $\left(\mathrm{CDCl}_{3}, 400 \mathrm{MHz}\right)$
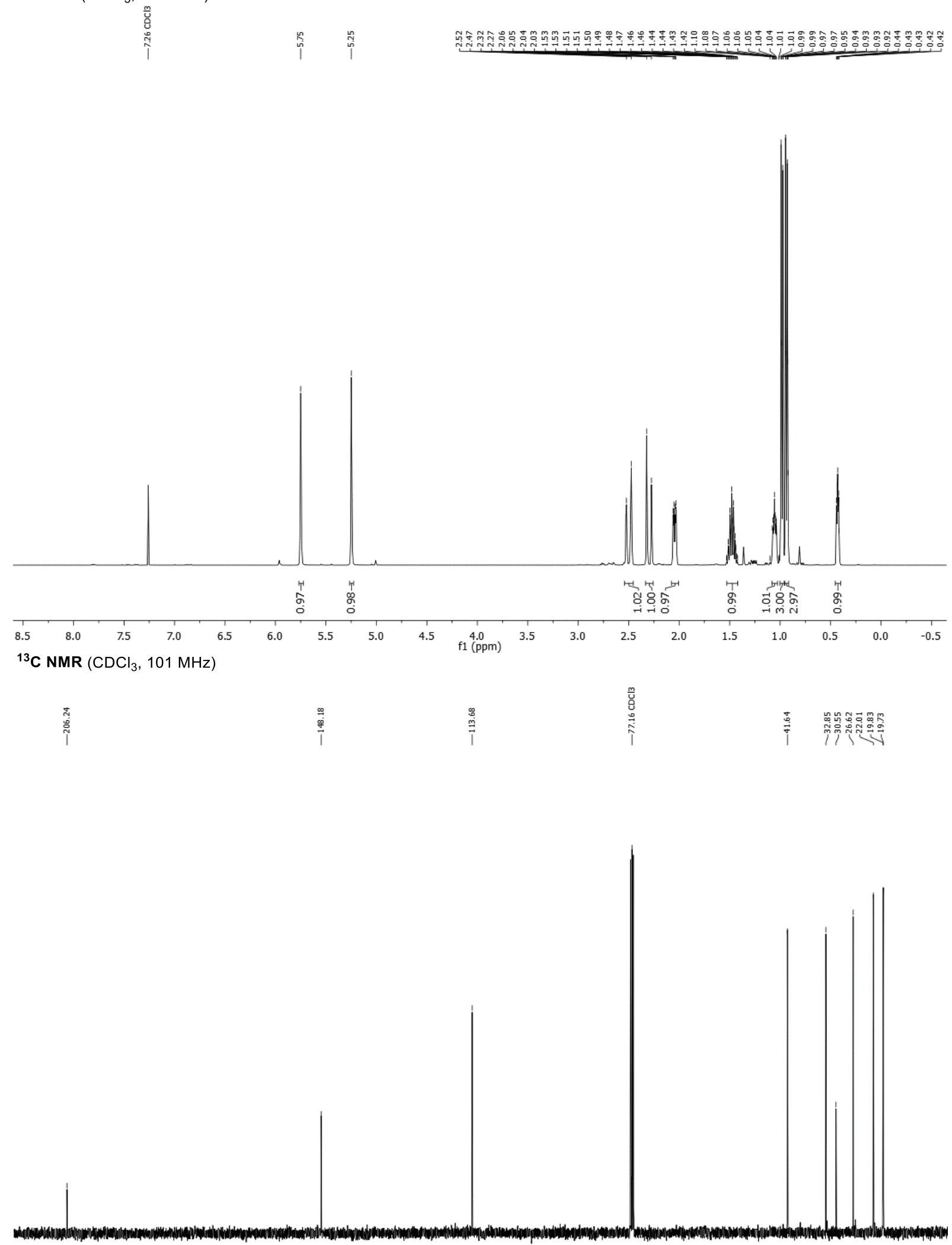

$\begin{array}{llllllllll}210 & 200 & 190 & 180 & 170 & 160 & 150 & 140 & 130 & 120 \\ \mathrm{f} 1(\mathrm{ppm})\end{array}$ 


$$
\text { EtOOC\/ }
$$

${ }^{1} \mathrm{H}$ NMR $\left(\mathrm{CDCl}_{3}, 400 \mathrm{MHz}\right)$

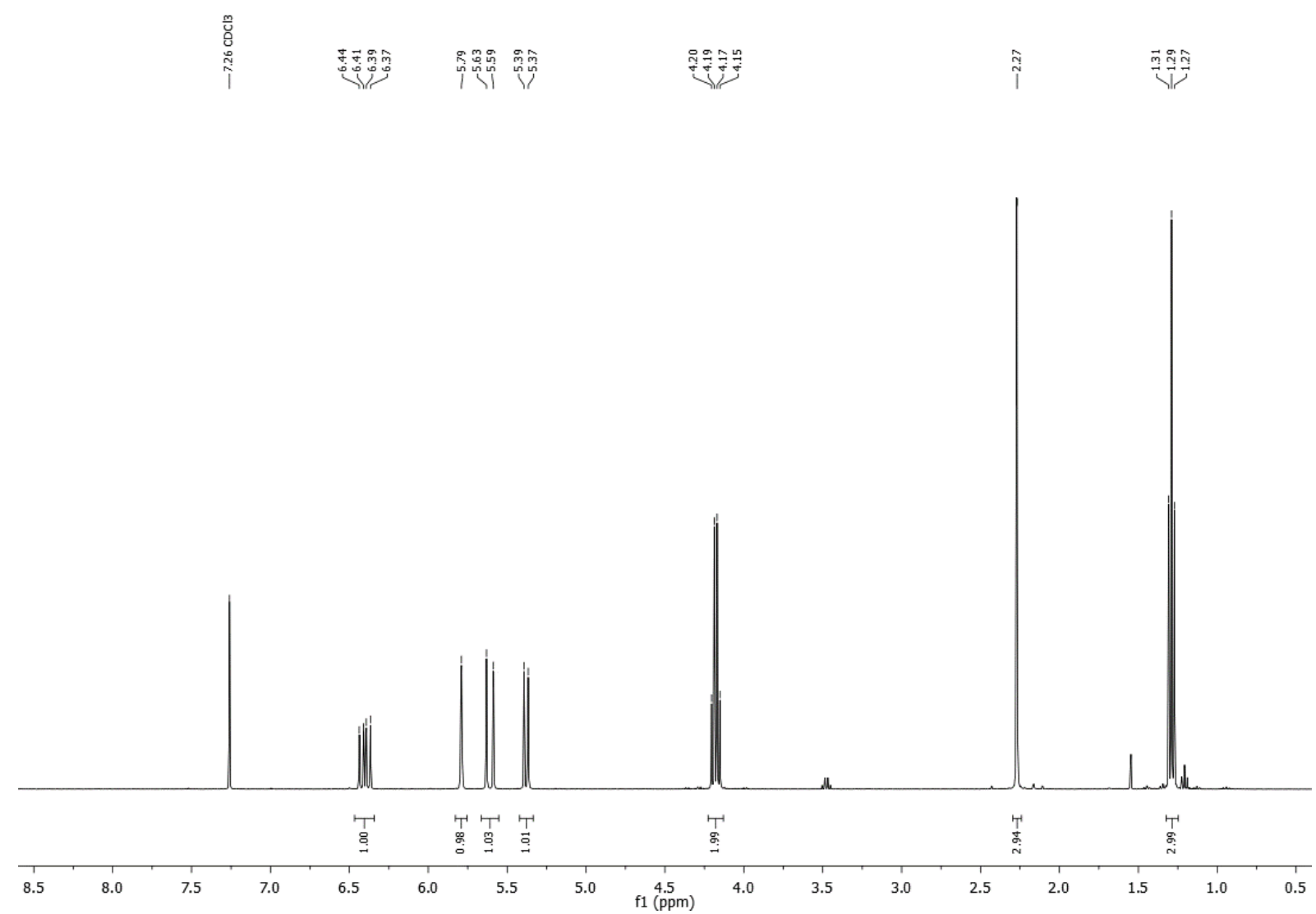

${ }^{13} \mathrm{C}$ NMR $\left(\mathrm{CDCl}_{3}, 101 \mathrm{MHz}\right)$

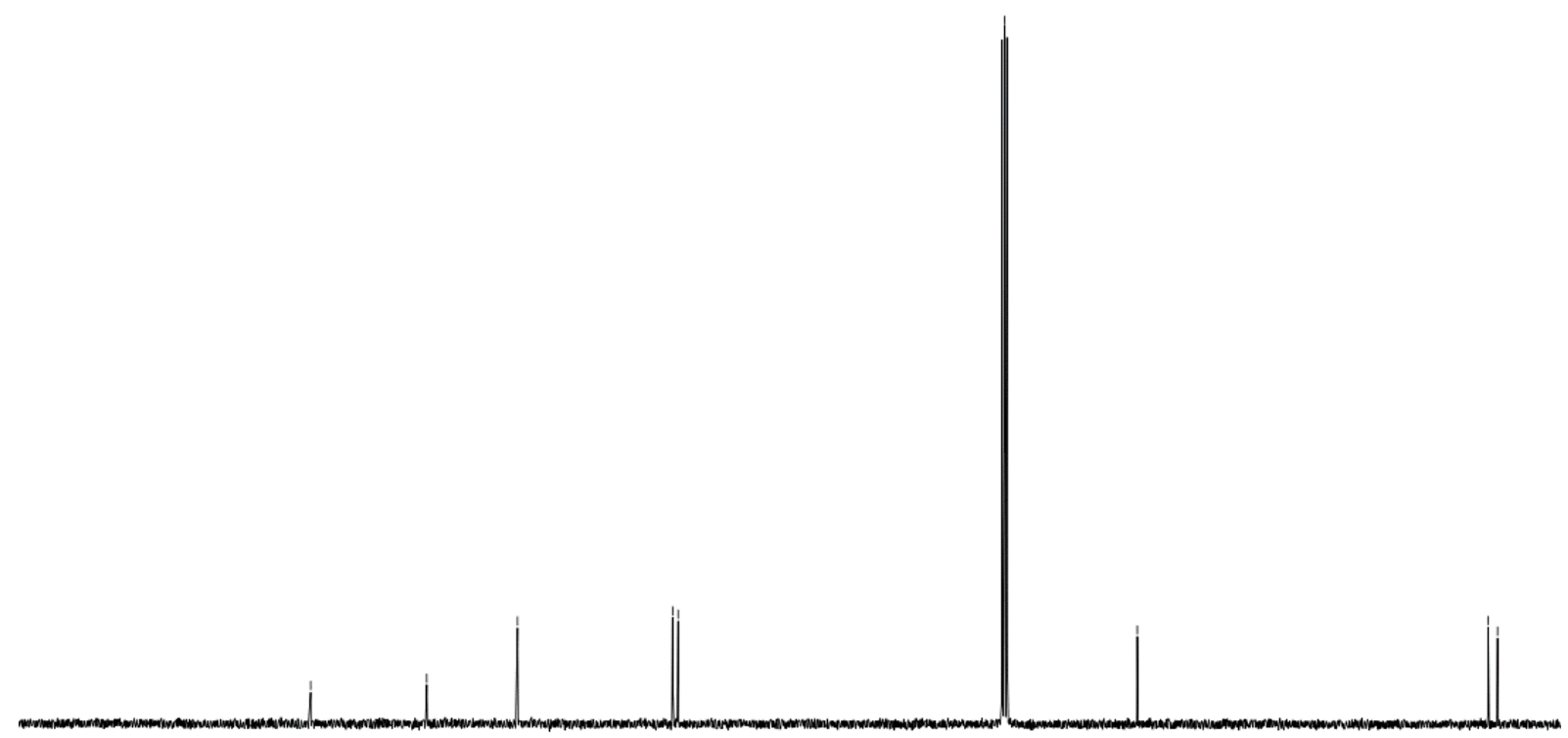

$\begin{array}{llllllllll}200 & 190 & 180 & 170 & 160 & 150 & 140 & 130 & 120 & 110 \\ \text { f1 (ppm) }\end{array}$ 
S-38

${ }^{1} \mathrm{H}$ NMR $\left(\mathrm{CDCl}_{3}, 400 \mathrm{MHz}\right)$

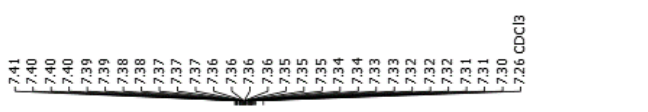

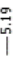
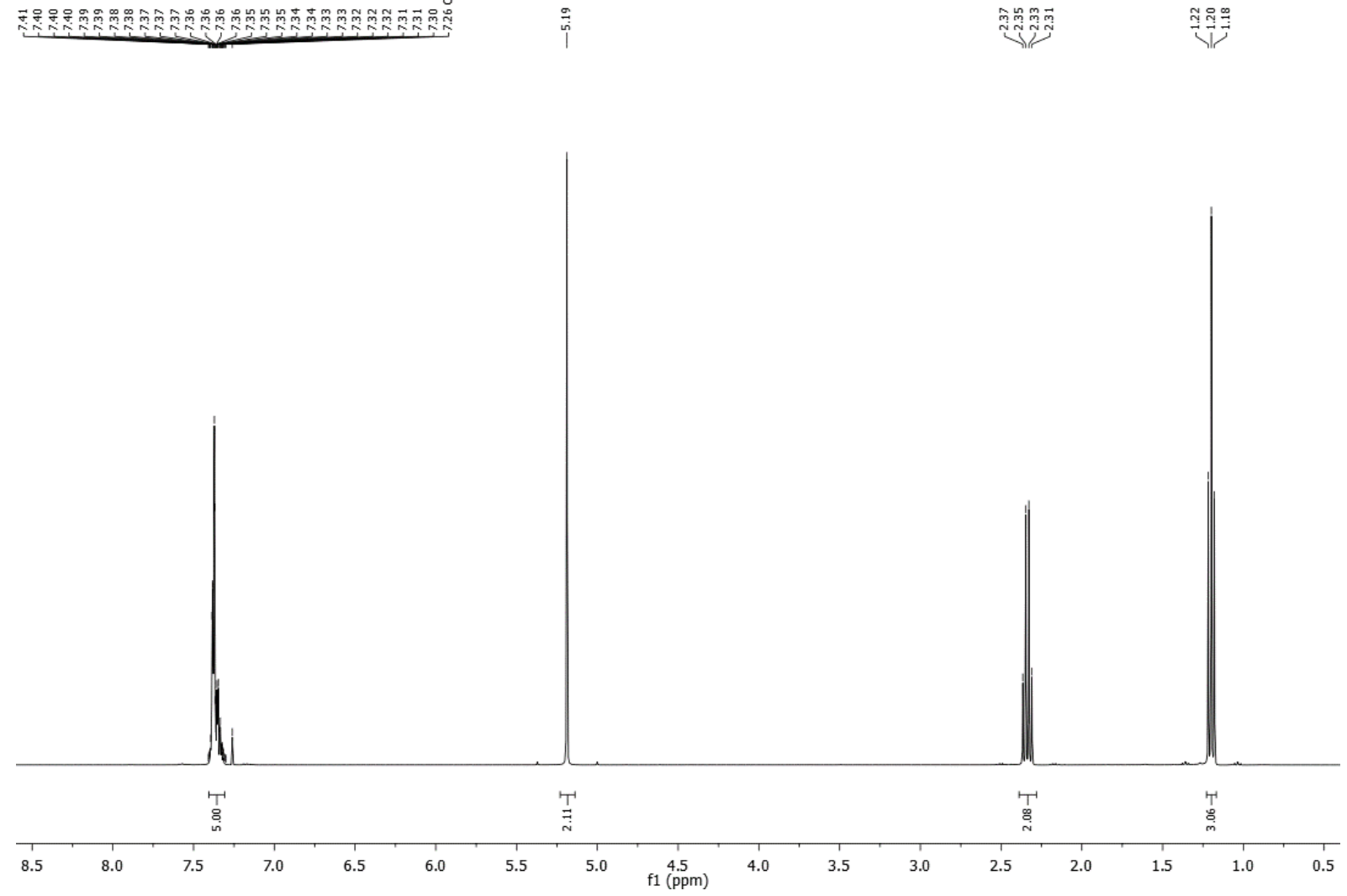

${ }^{13} \mathrm{C} \mathrm{NMR}\left(\mathrm{CDCl}_{3}, 101 \mathrm{MHz}\right)$

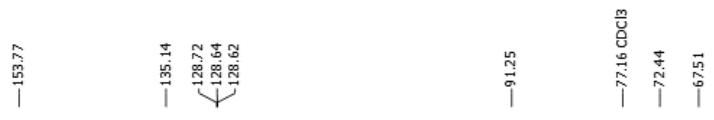

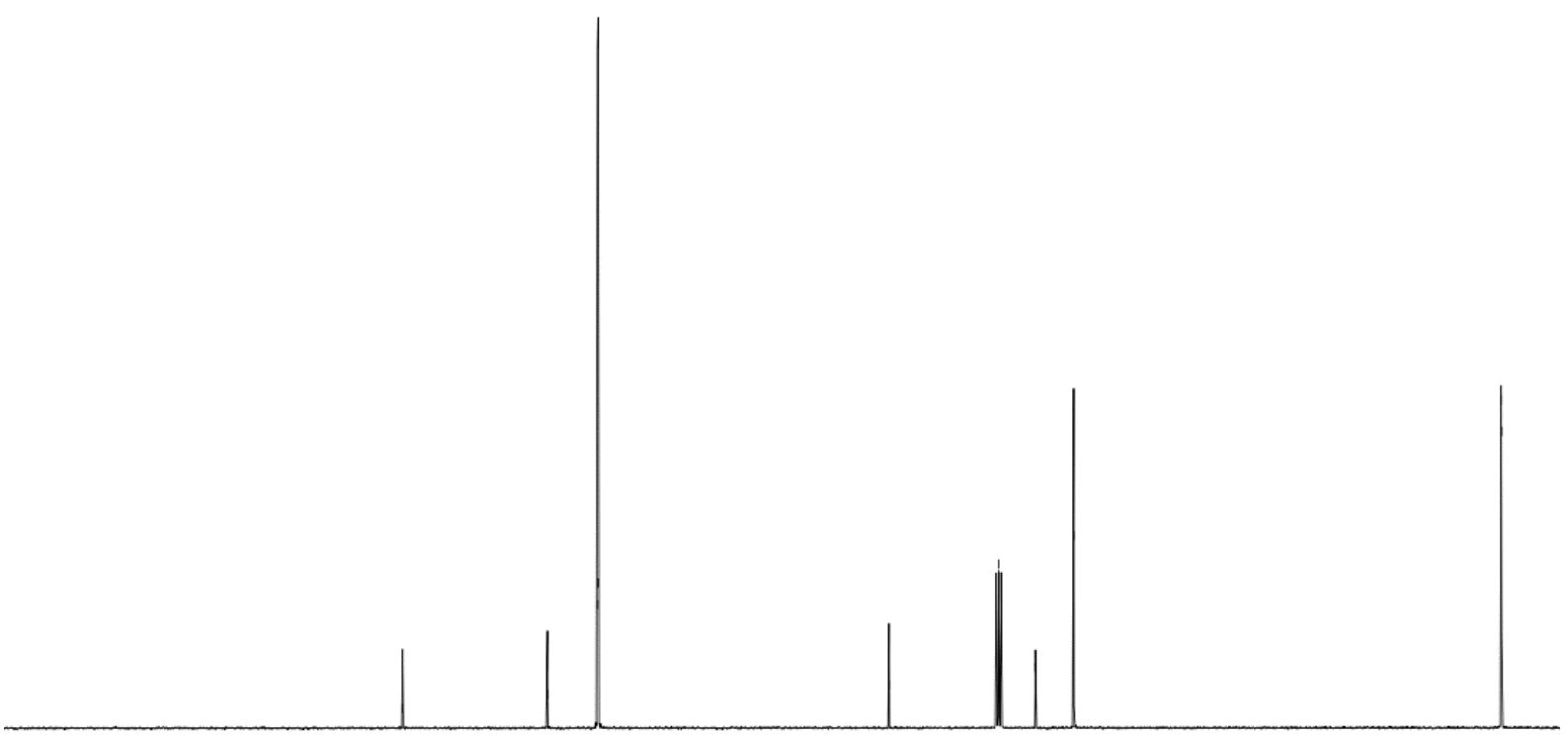




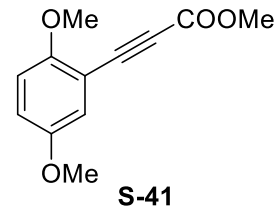

${ }^{1} \mathrm{H}$ NMR $\left(\mathrm{CDCl}_{3}, 400 \mathrm{MHz}\right)$
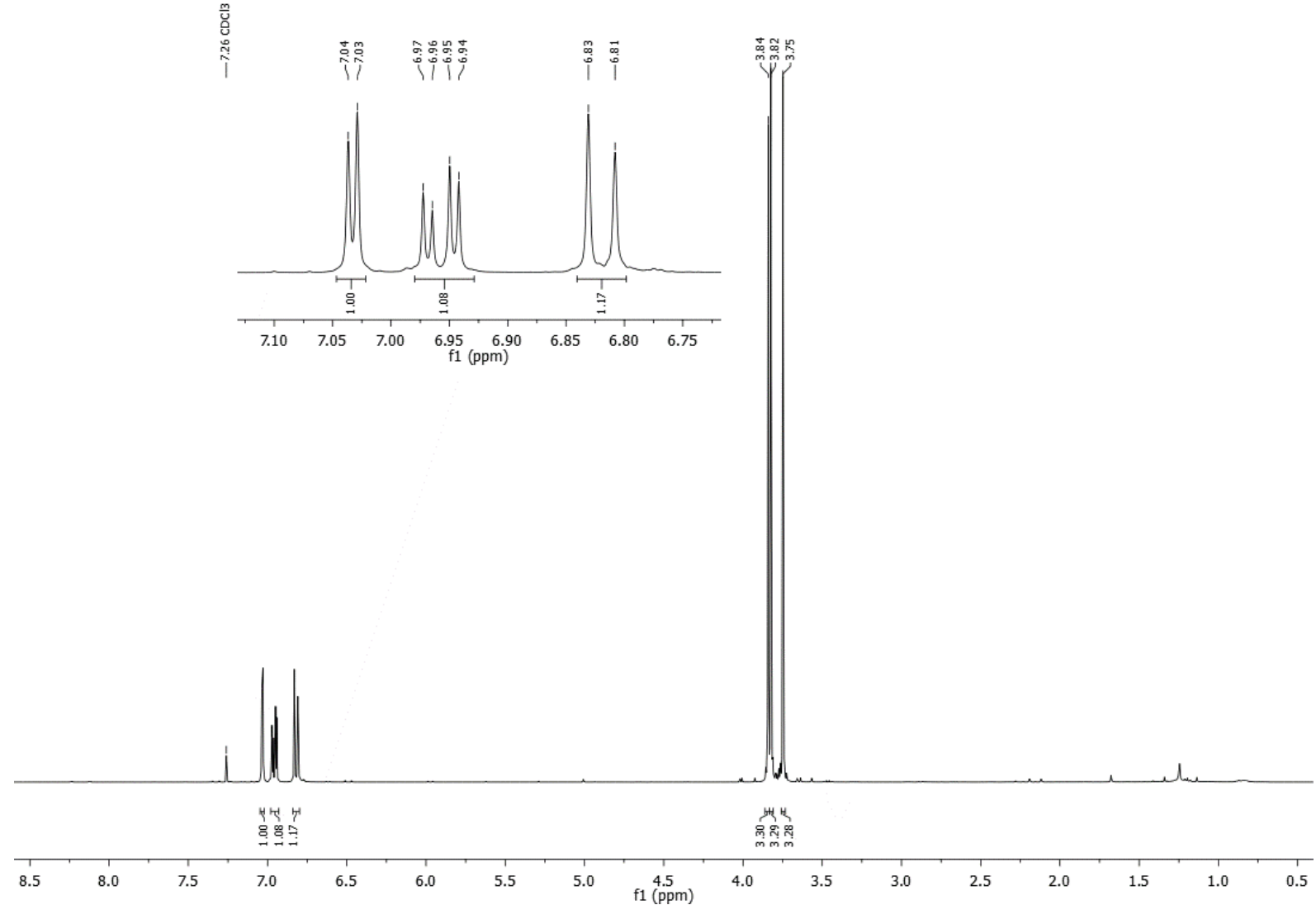

${ }^{13} \mathrm{C}$ NMR $\left(\mathrm{CDCl}_{3}, 101 \mathrm{MHz}\right)$

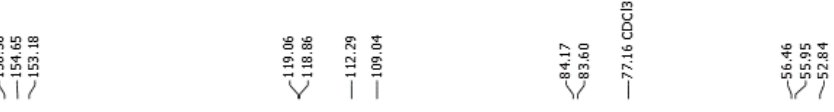

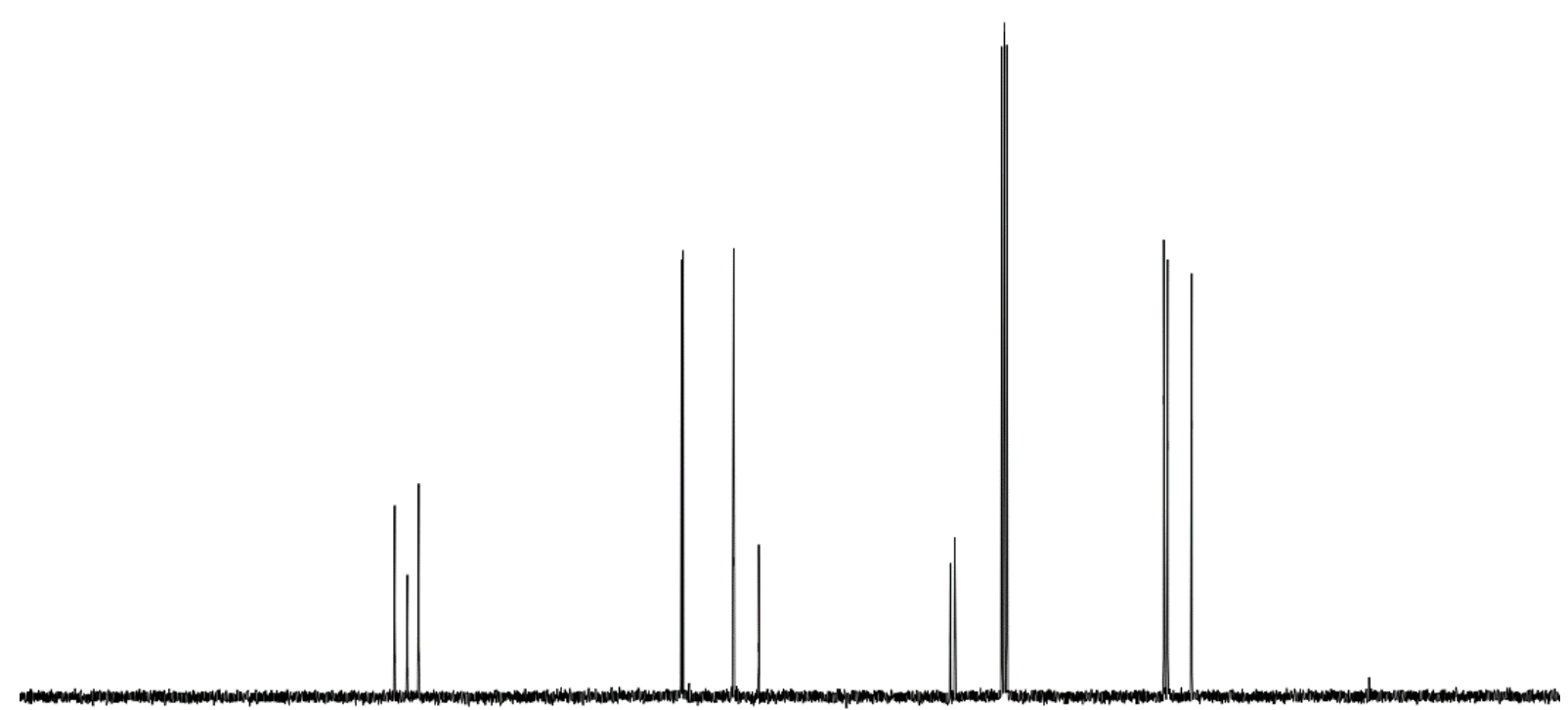

$\begin{array}{llllllllll}200 & 190 & 180 & 170 & 160 & 150 & 140 & 130 & 120 & 110 \\ \mathrm{f} 1(\mathrm{ppm})\end{array}$ 


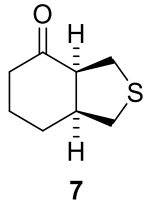

${ }^{1} \mathrm{H} \mathrm{NMR}\left(\mathrm{CDCl}_{3}, 400 \mathrm{MHz}\right)$
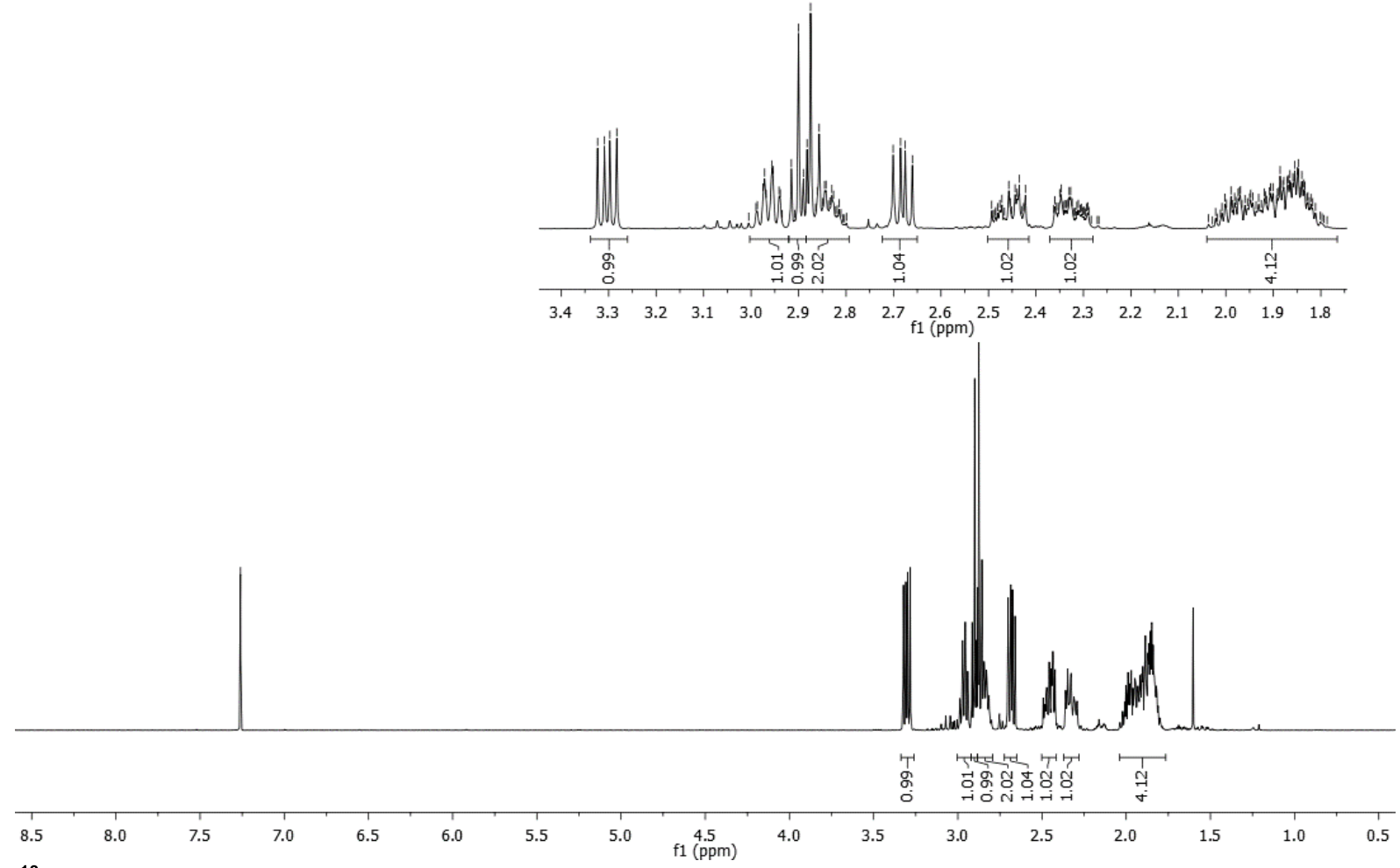

${ }^{13} \mathrm{C}$ NMR $\left(\mathrm{CDCl}_{3}, 101 \mathrm{MHz}\right)$ 


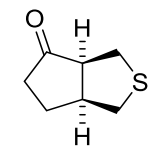

$8 \mathbf{a}$

${ }^{1} \mathrm{H} \mathrm{NMR}\left(\mathrm{CDCl}_{3}, 400 \mathrm{MHz}\right)$
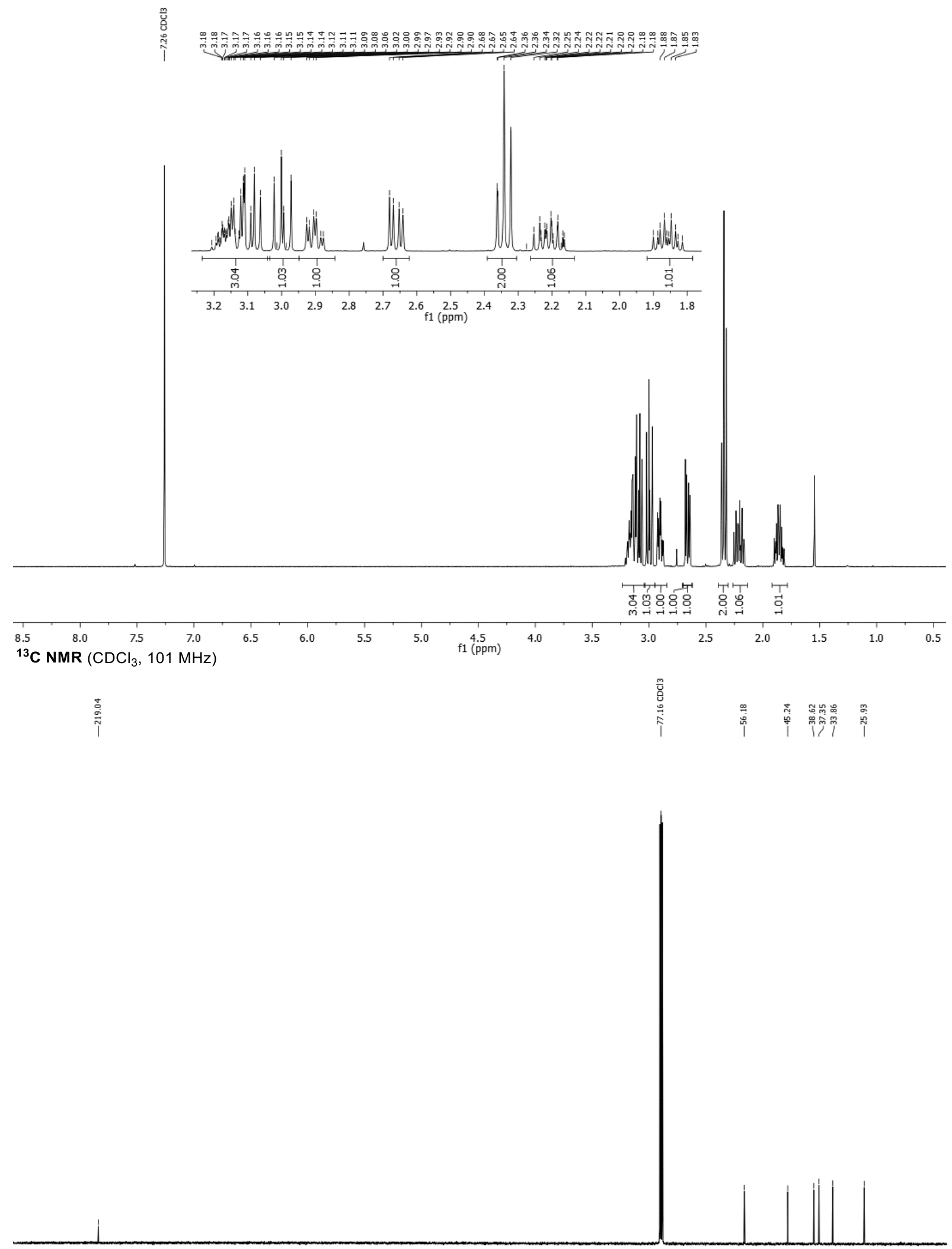

\begin{tabular}{llllllllllllllllllllllllll}
\hline 40 & 230 & 220 & 210 & 200 & 190 & 180 & 170 & 160 & 150 & 140 & 130 & 120 & 110 & 100 & 90 & 80 & 70 & 60 & 50 & 40 & 30 & 20 & 10
\end{tabular} 


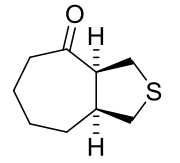

$8 \mathbf{b}$

${ }^{1} \mathrm{H}$ NMR $\left(\mathrm{CDCl}_{3}, 400 \mathrm{MHz}\right)$
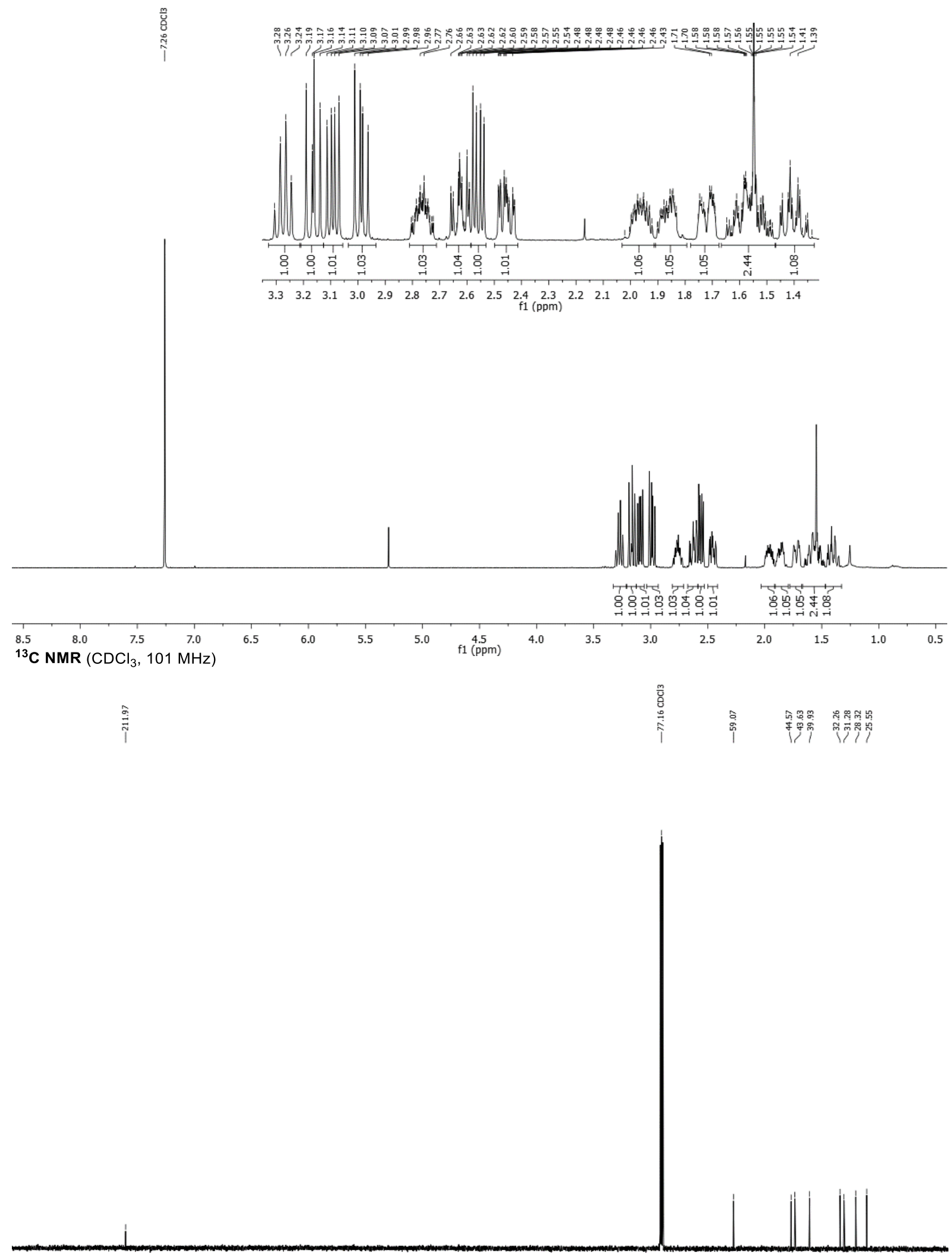

$\begin{array}{rllllllllllllllllllllll}40 & 230 & 220 & 210 & 200 & 190 & 180 & 170 & 160 & 150 & 140 & 130 \begin{array}{c}120 \\ (\mathrm{ppm})\end{array} & 110 & 100 & 90 & 80 & 70 & 60 & 50 & 40 & 30 & 20 & 10\end{array}$ 


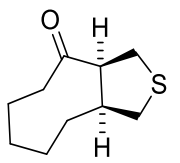

$8 \mathrm{c}$

${ }^{1} \mathrm{H}$ NMR $\left(\mathrm{CDCl}_{3}, 400 \mathrm{MHz}\right)$

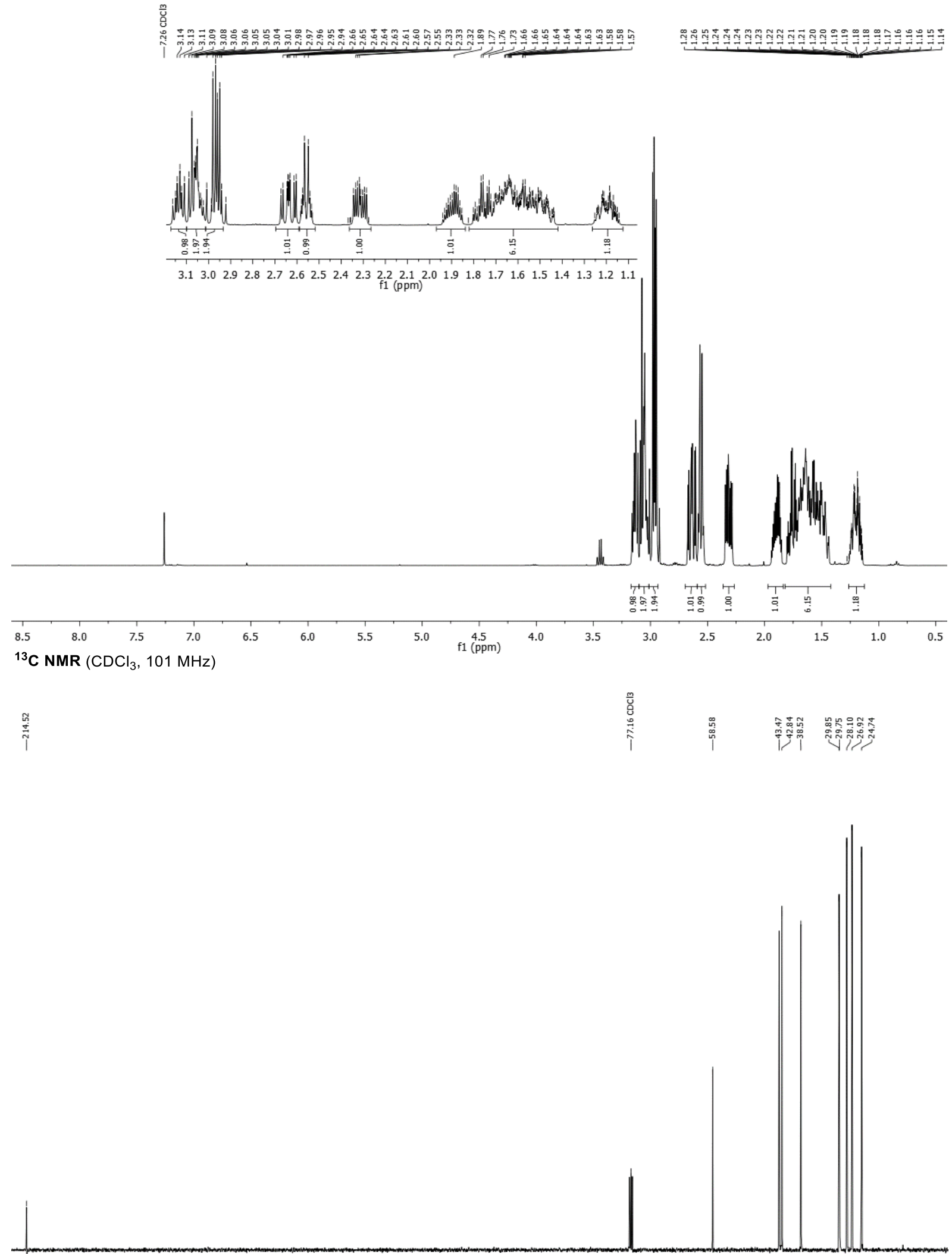

$\begin{array}{lllllllllllllllllllll}210 & 200 & 190 & 180 & 170 & 160 & 150 & 140 & 130 & 120 & \begin{array}{l}110 \\ \mathrm{f} 1(\mathrm{ppm})\end{array} & 100 & 90 & 80 & 70 & 60 & 50 & 40 & 30 & 20 & 10\end{array}$ 


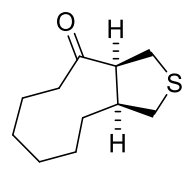

$8 d$

${ }^{1} \mathrm{H}$ NMR $\left(\mathrm{CDCl}_{3}, 400 \mathrm{MHz}\right)$
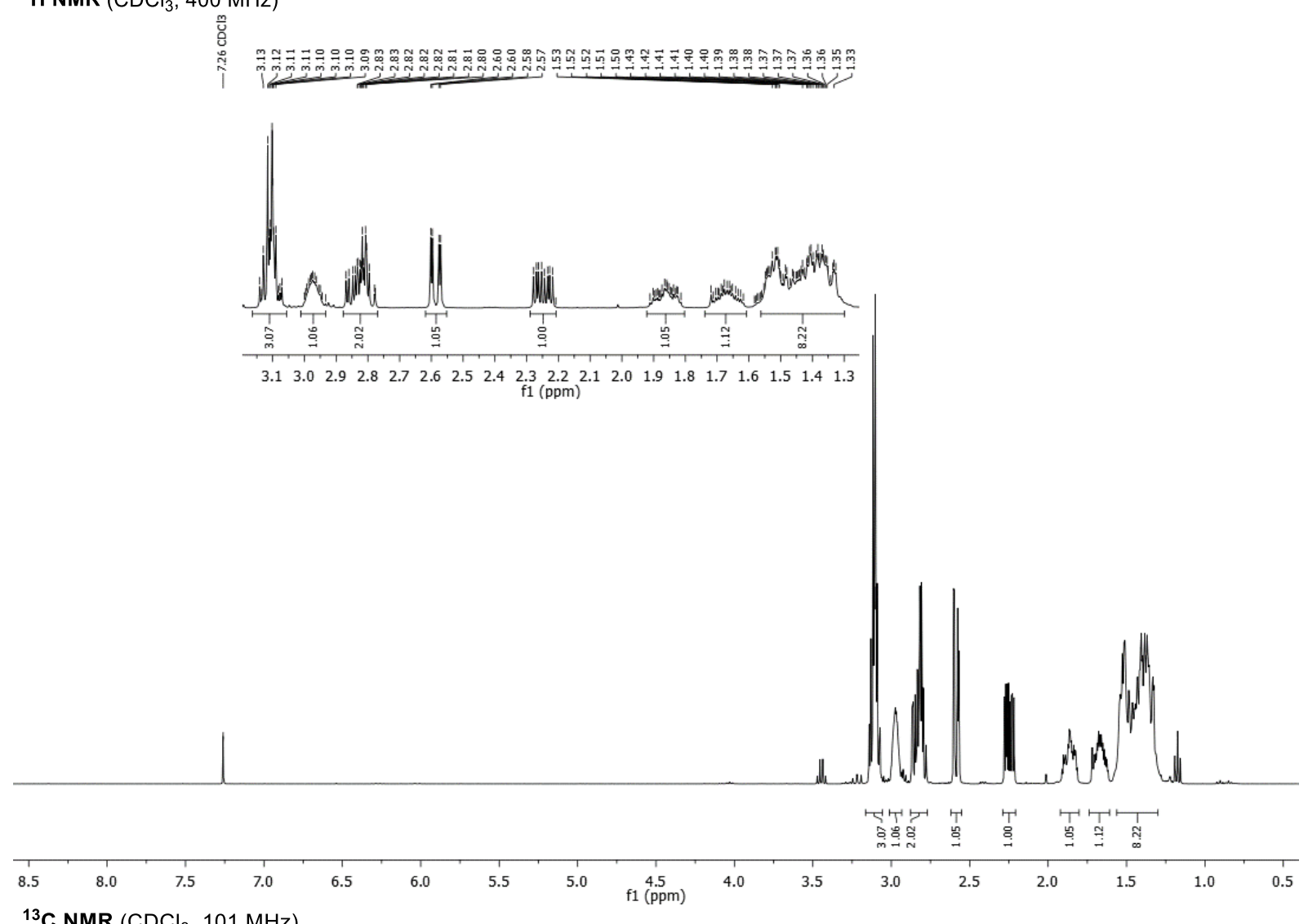

${ }^{13} \mathrm{C}$ NMR $\left(\mathrm{CDCl}_{3}, 101 \mathrm{MHz}\right)$

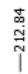
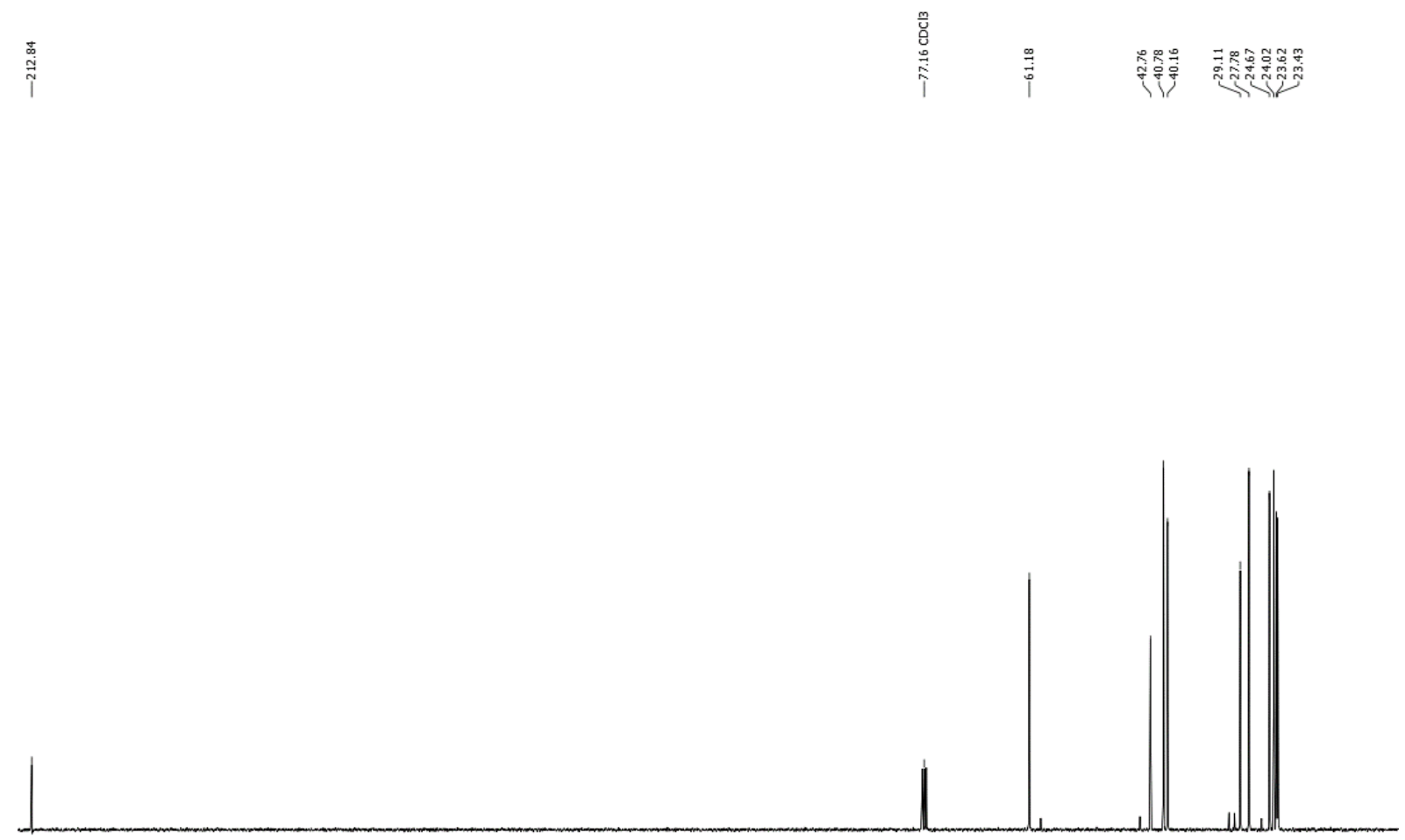

210

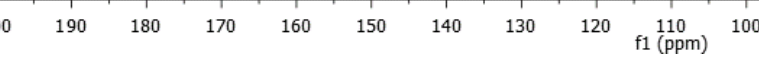




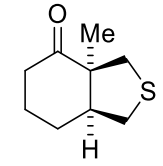

$8 \mathrm{e}$

${ }^{1} \mathrm{H}$ NMR $\left(\mathrm{CDCl}_{3}, 599 \mathrm{MHz}\right)$

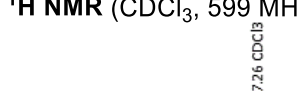
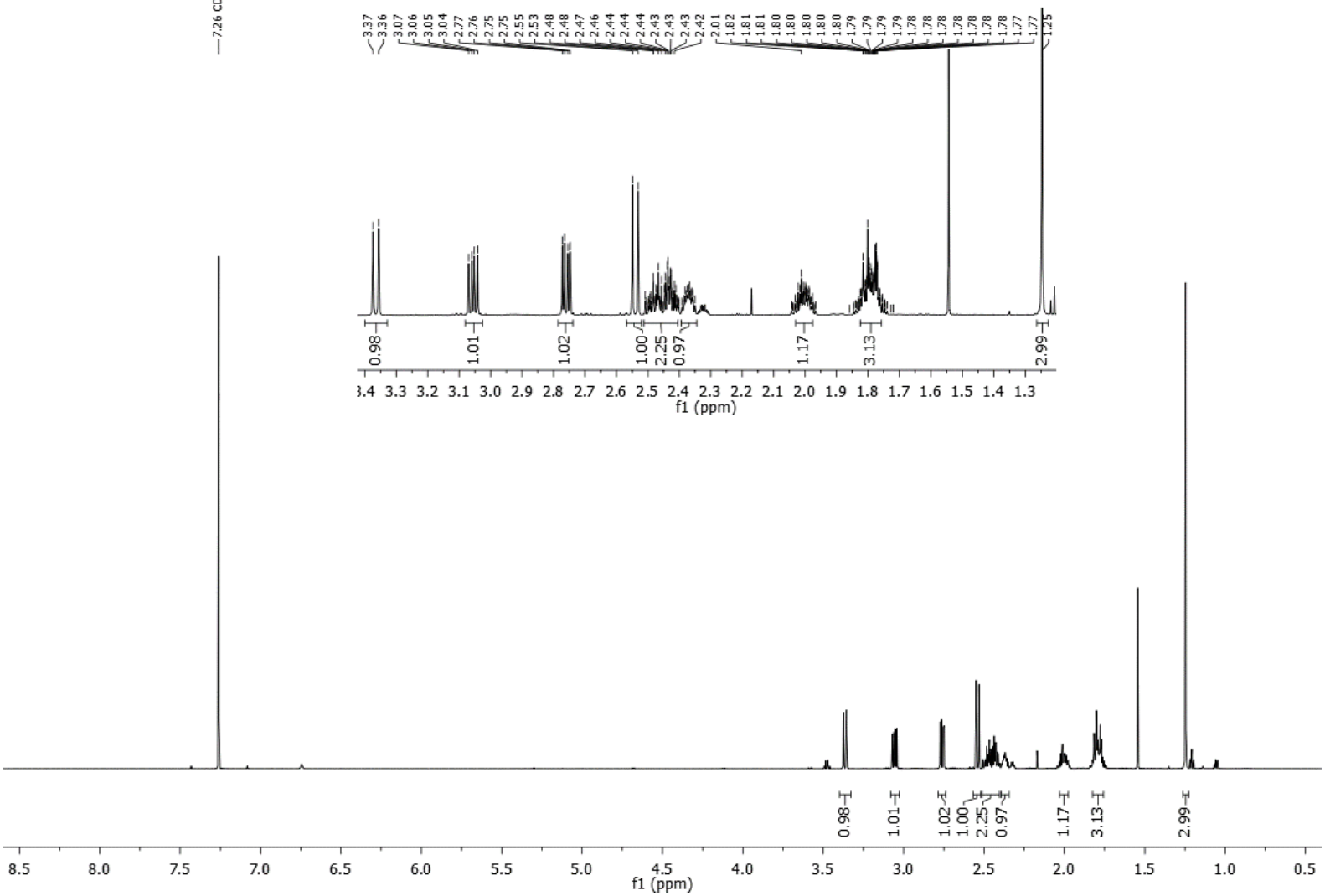

${ }^{13} \mathrm{C}$ NMR $\left(\mathrm{CDCl}_{3}, 151 \mathrm{MHz}\right)$

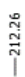

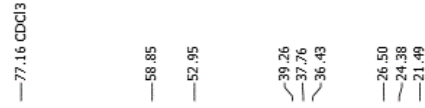

$\begin{array}{lllllllllll}220 & 210 & 200 & 190 & 180 & 170 & 160 & 150 & 140 & 130 & 120 \quad 110 \\ (\mathrm{ppm})\end{array}$ 


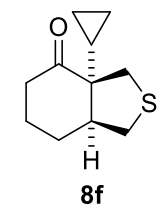

${ }^{1} \mathrm{H}$ NMR $\left(\mathrm{CDCl}_{3}, 400 \mathrm{MHz}\right)$
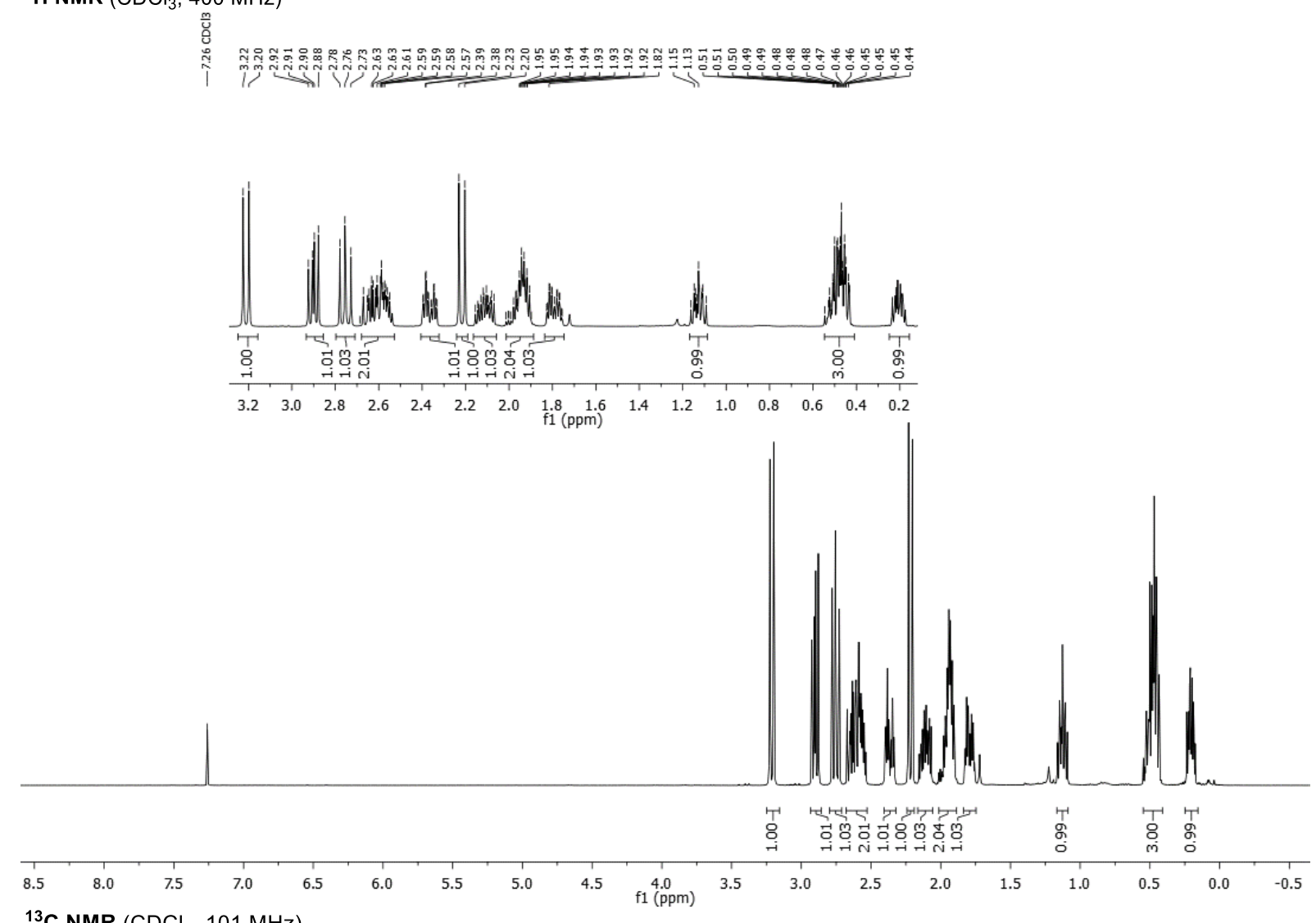

${ }^{13} \mathrm{C}$ NMR $\left(\mathrm{CDCl}_{3}, 101 \mathrm{MHz}\right)$ 


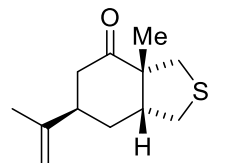

$(+)-\mathbf{8 g}$

${ }^{1} \mathrm{H}$ NMR $\left(\mathrm{CDCl}_{3}, 400 \mathrm{MHz}\right)$

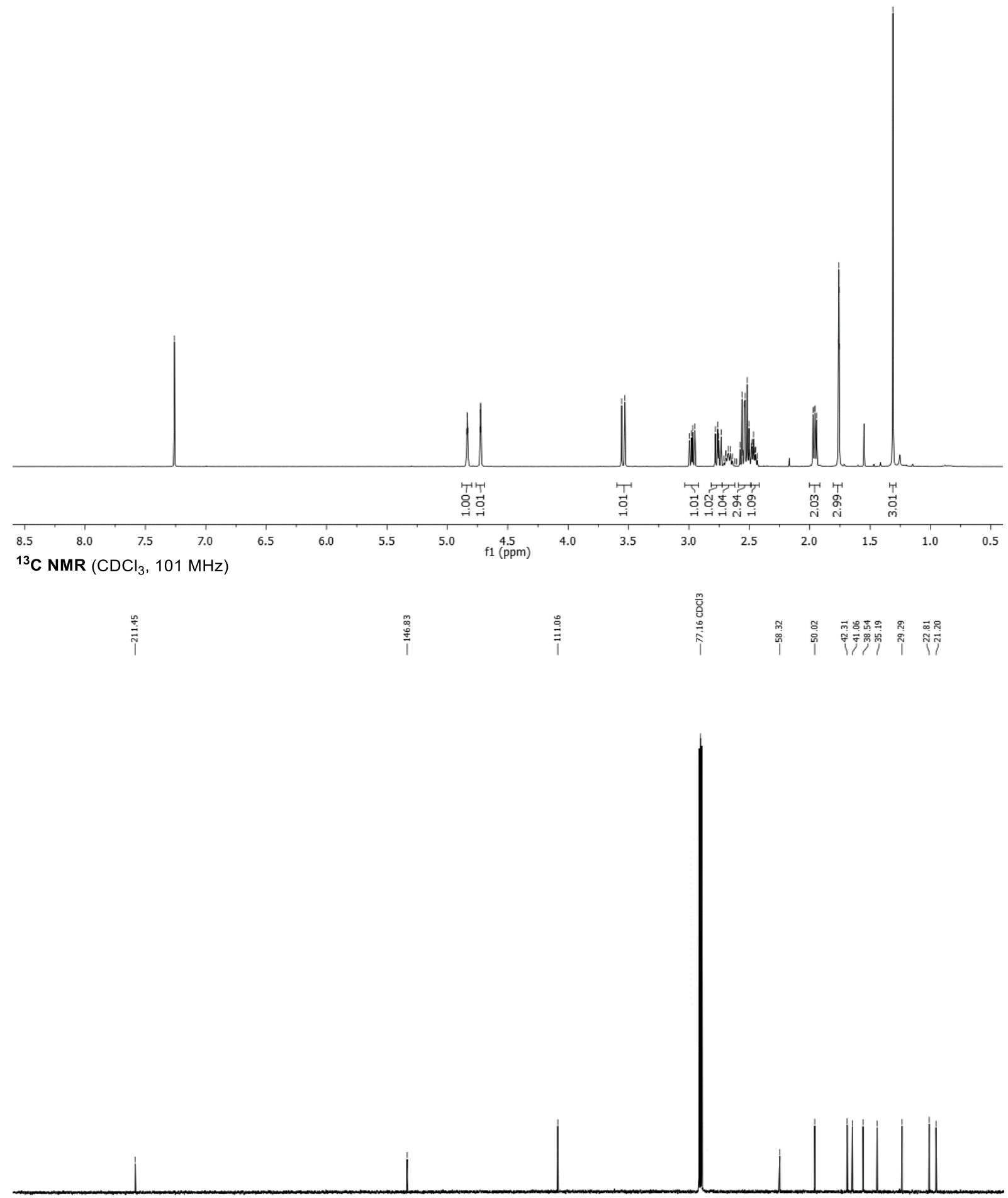

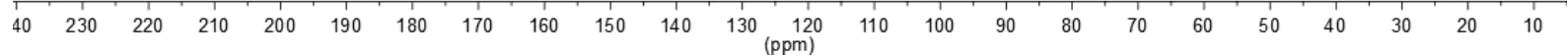




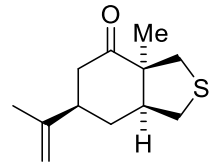

(+)-S-42

${ }^{1} \mathrm{H}$ NMR $\left(\mathrm{CDCl}_{3}, 599 \mathrm{MHz}\right)$

熵

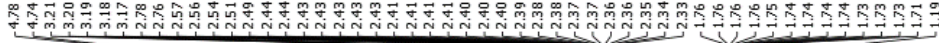

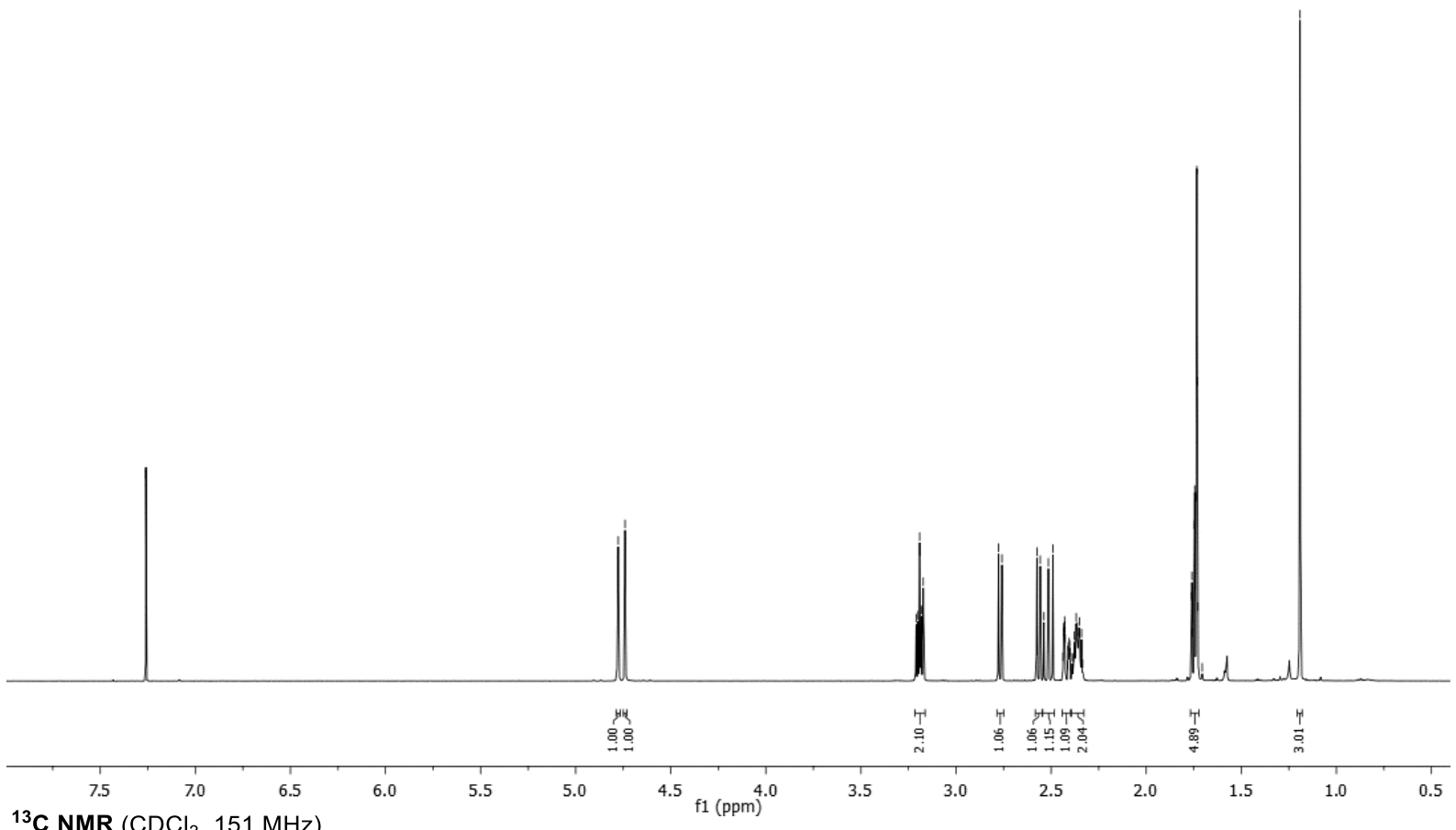

${ }^{13} \mathbf{C}$ NMR $\left(\mathrm{CDCl}_{3}, 151 \mathrm{MHz}\right)$

$\stackrel{\text { ํำ }}{\stackrel{N}{1}}$

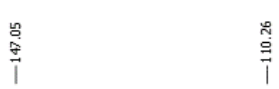

$\frac{m}{u}$
0
0
0
$i$
$i$

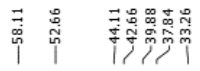

กั้

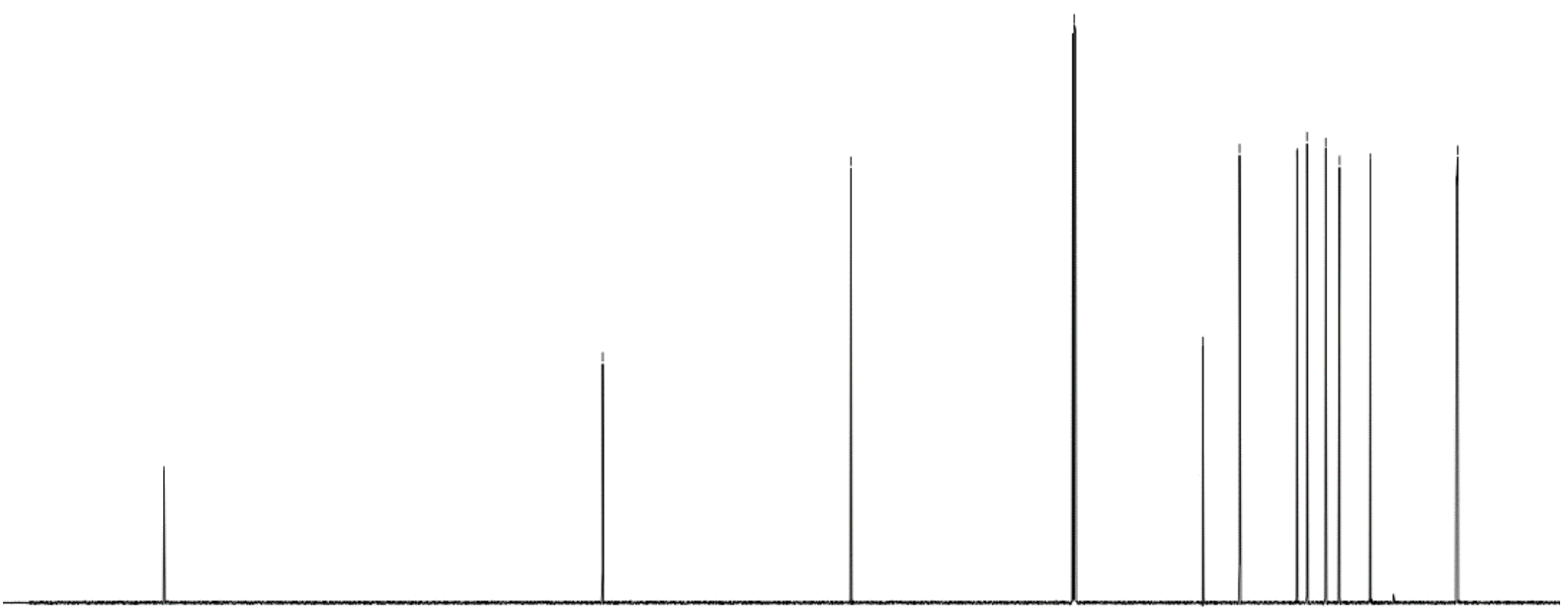

$230 \quad 2$ $210 \quad 200$ $\begin{array}{llllll}190 & 180 & 170 & 160 & 150 & 140\end{array}$ $30 \begin{gathered}120 \\ \mathrm{f} 1(\mathrm{ppm})\end{gathered}$ 
<smiles>CC1(C)CCC(=O)C2CCCC21</smiles>

$8 \mathbf{h}$

${ }^{1} \mathbf{H}$ NMR $\left(\mathrm{CDCl}_{3}, 599 \mathrm{MHz}\right)$
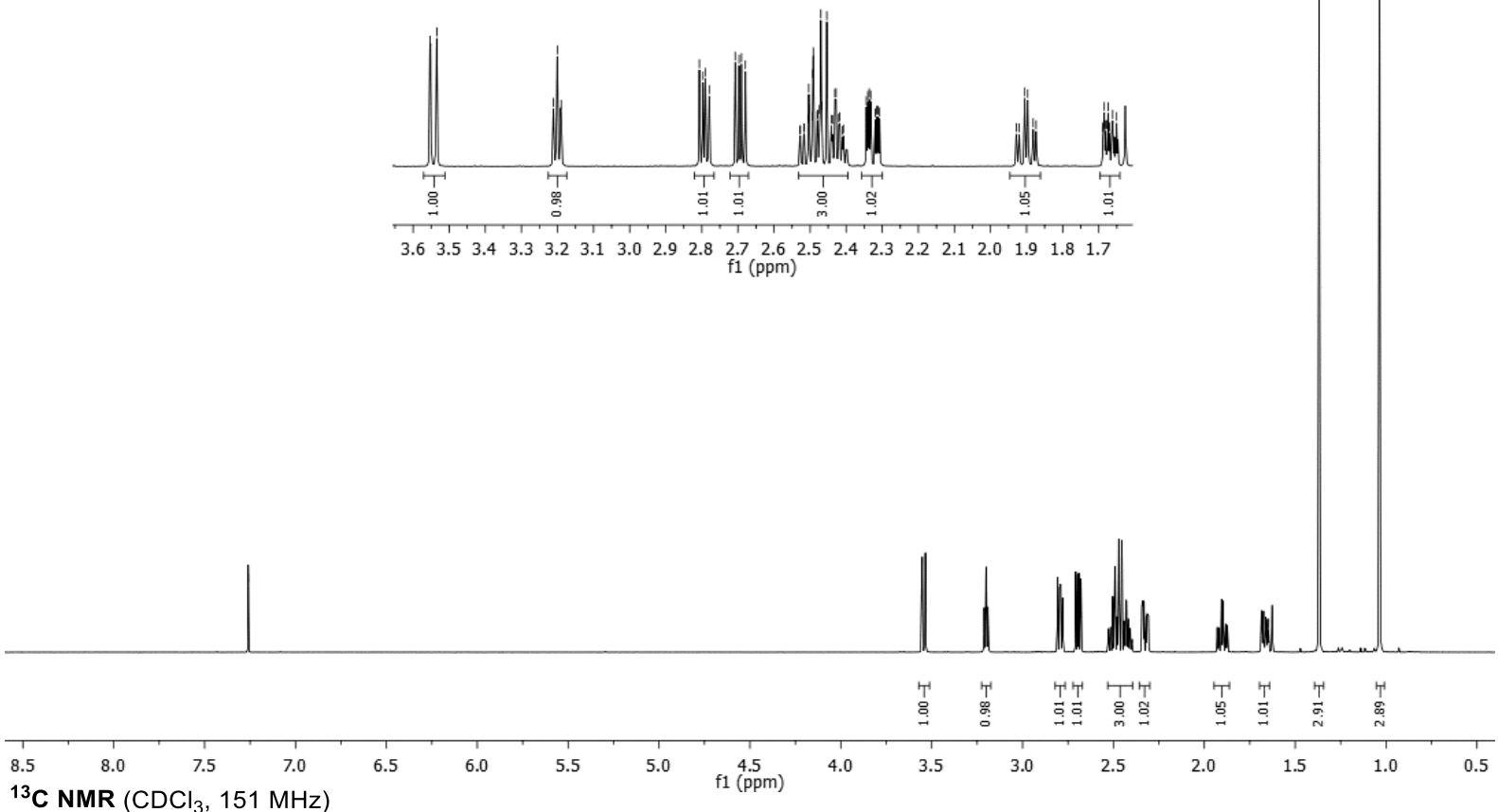


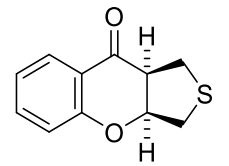

$8 \mathbf{i}$

${ }^{1} \mathrm{H}$ NMR $\left(\mathrm{CDCl}_{3}, 400 \mathrm{MHz}\right)$
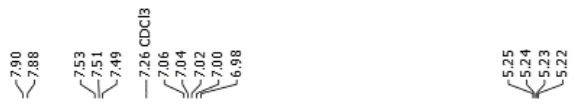

$\stackrel{m}{m} \stackrel{m}{m}$

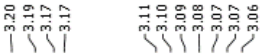

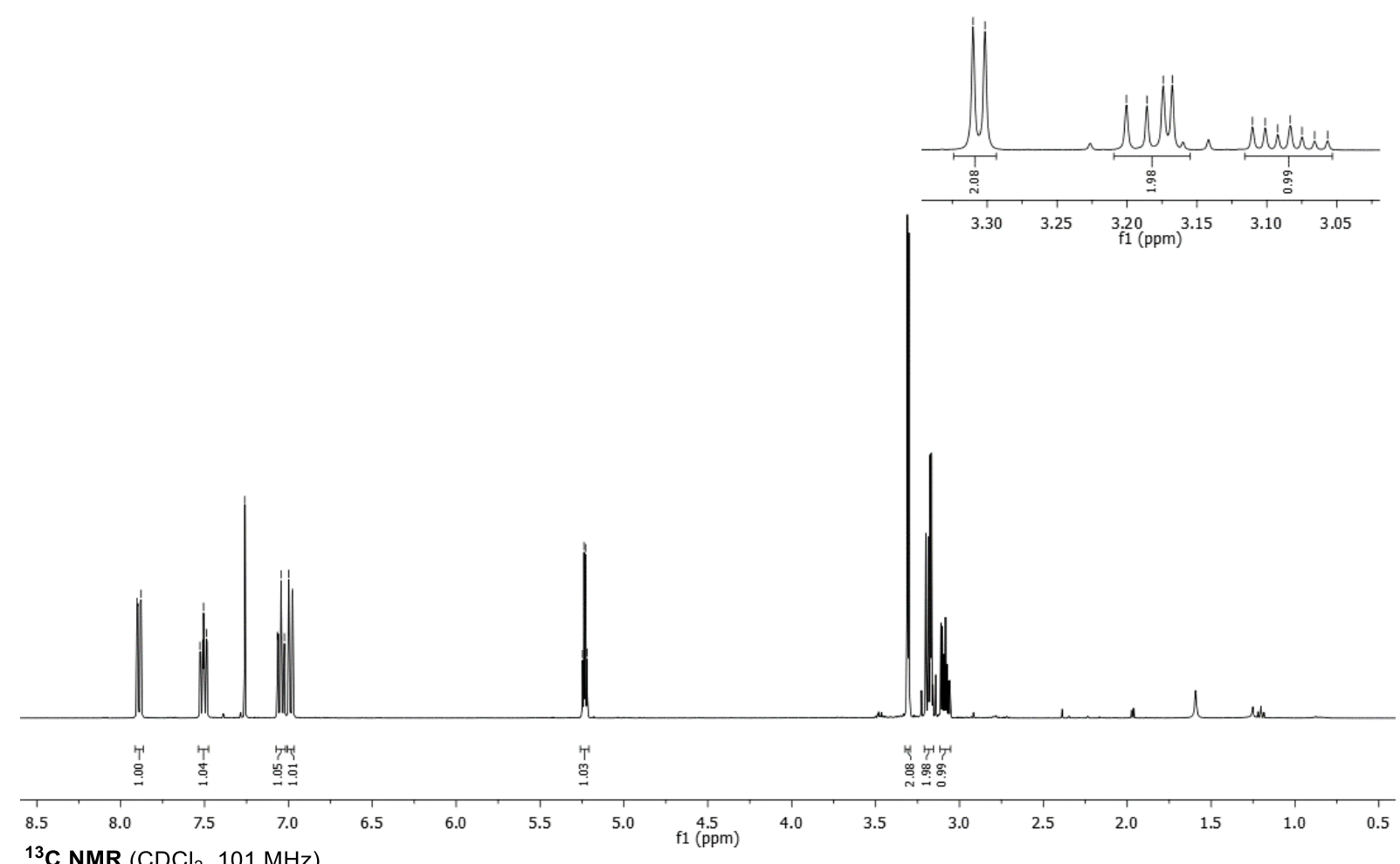

${ }^{13} \mathrm{C}$ NMR $\left(\mathrm{CDCl}_{3}, 101 \mathrm{MHz}\right)$

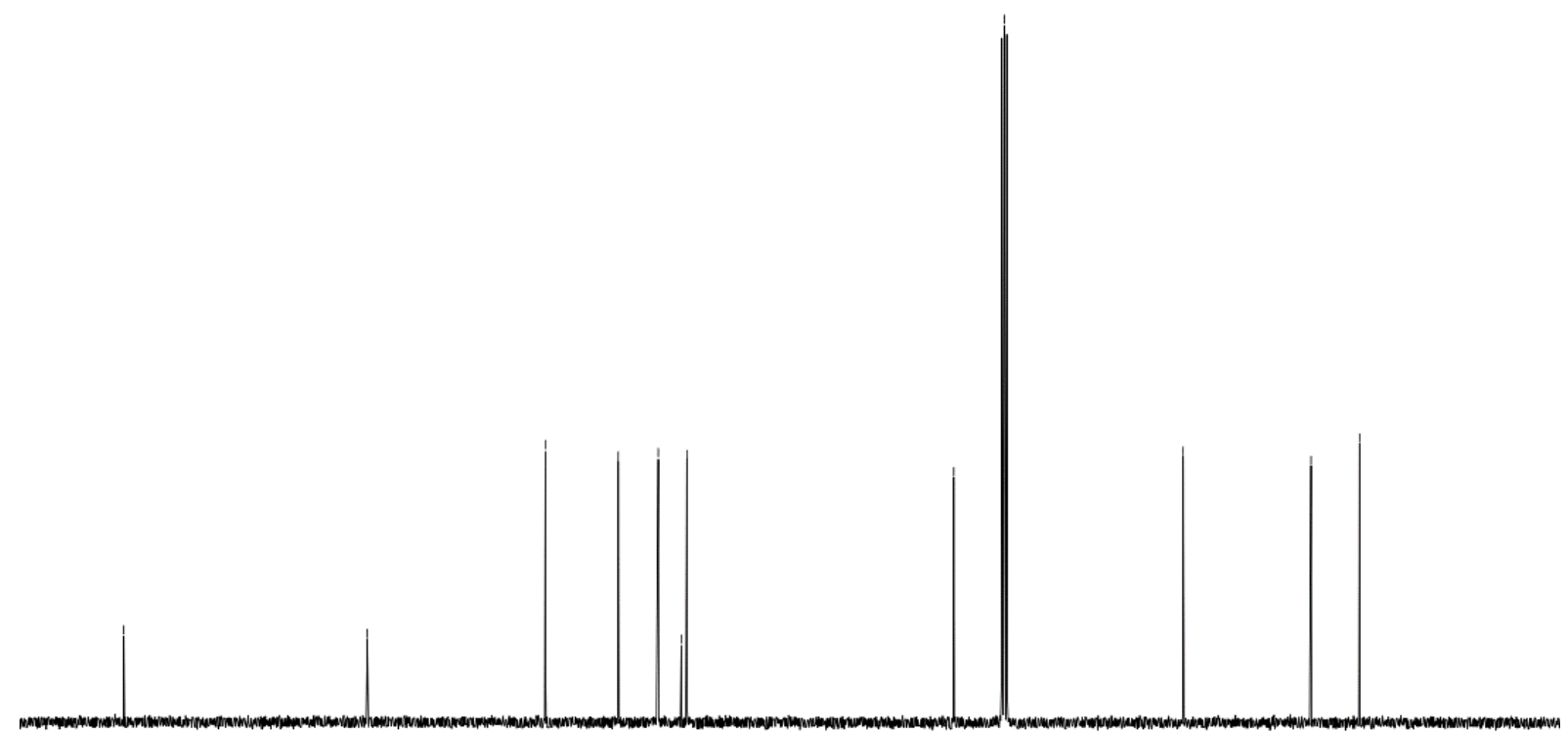




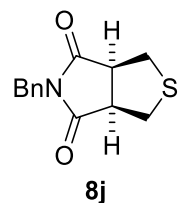

${ }^{1} \mathrm{H}$ NMR $\left(\mathrm{CDCl}_{3}, 400 \mathrm{MHz}\right)$

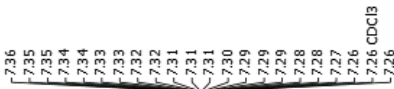

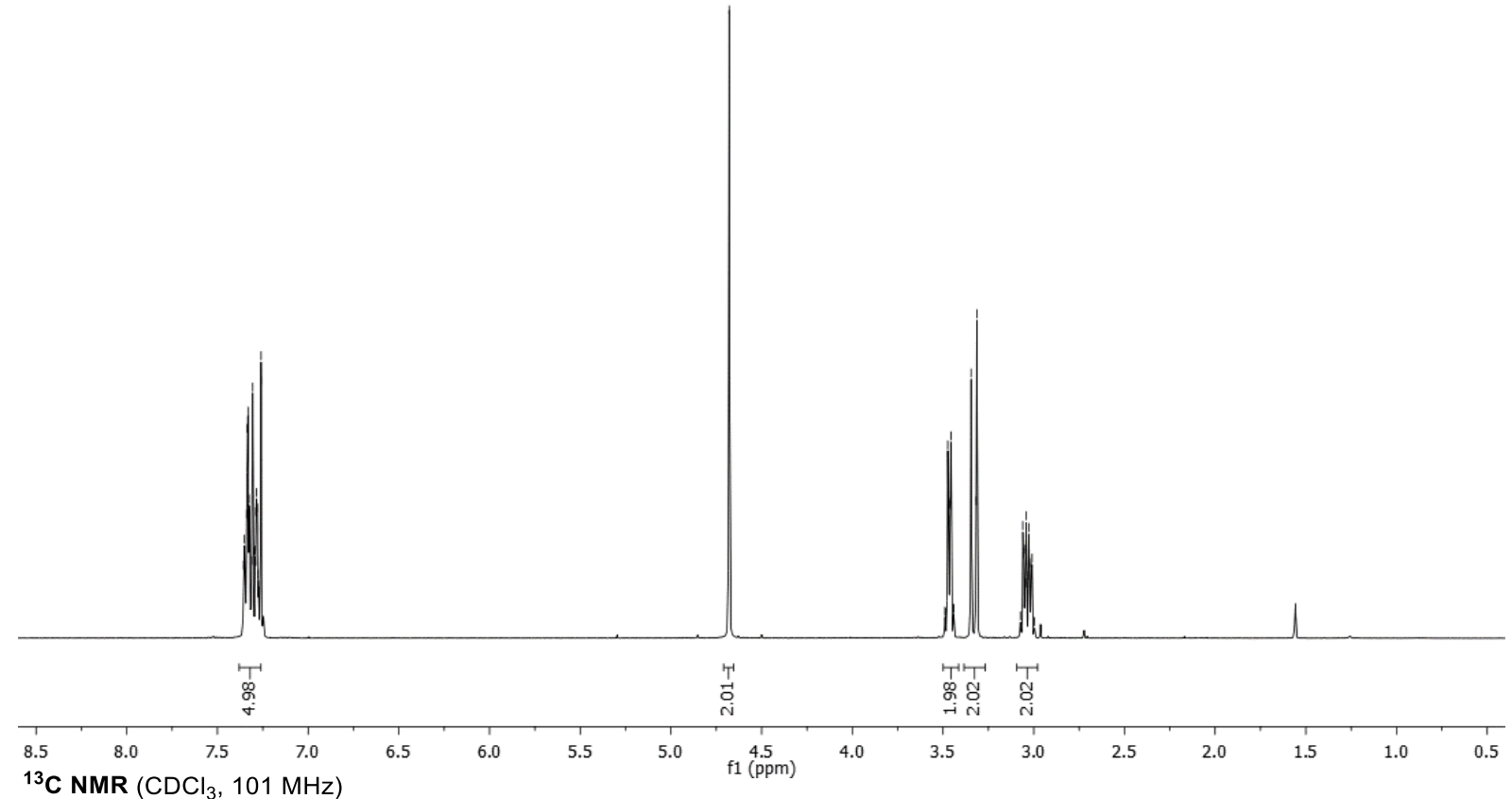

${ }^{13} \mathrm{C}$ NMR $\left(\mathrm{CDCl}_{3}, 101 \mathrm{MHz}\right)$
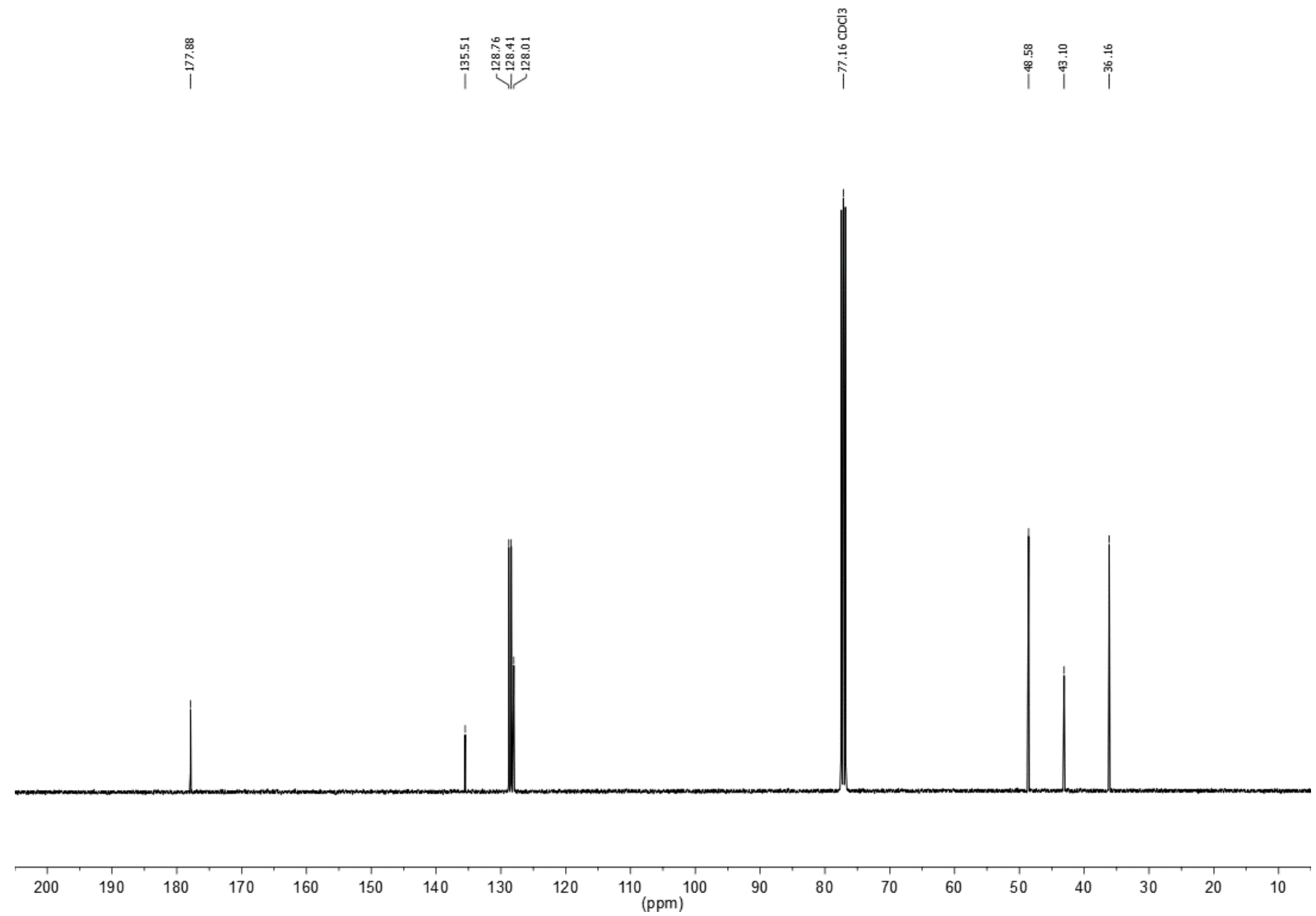
<smiles>C=C(C)C1CCC2(CO)CSCC2C1</smiles>

$(+)-8 \mathbf{k}$

${ }^{1} \mathrm{H}$ NMR $\left(\mathrm{CDCl}_{3}, 400 \mathrm{MHz}\right)$

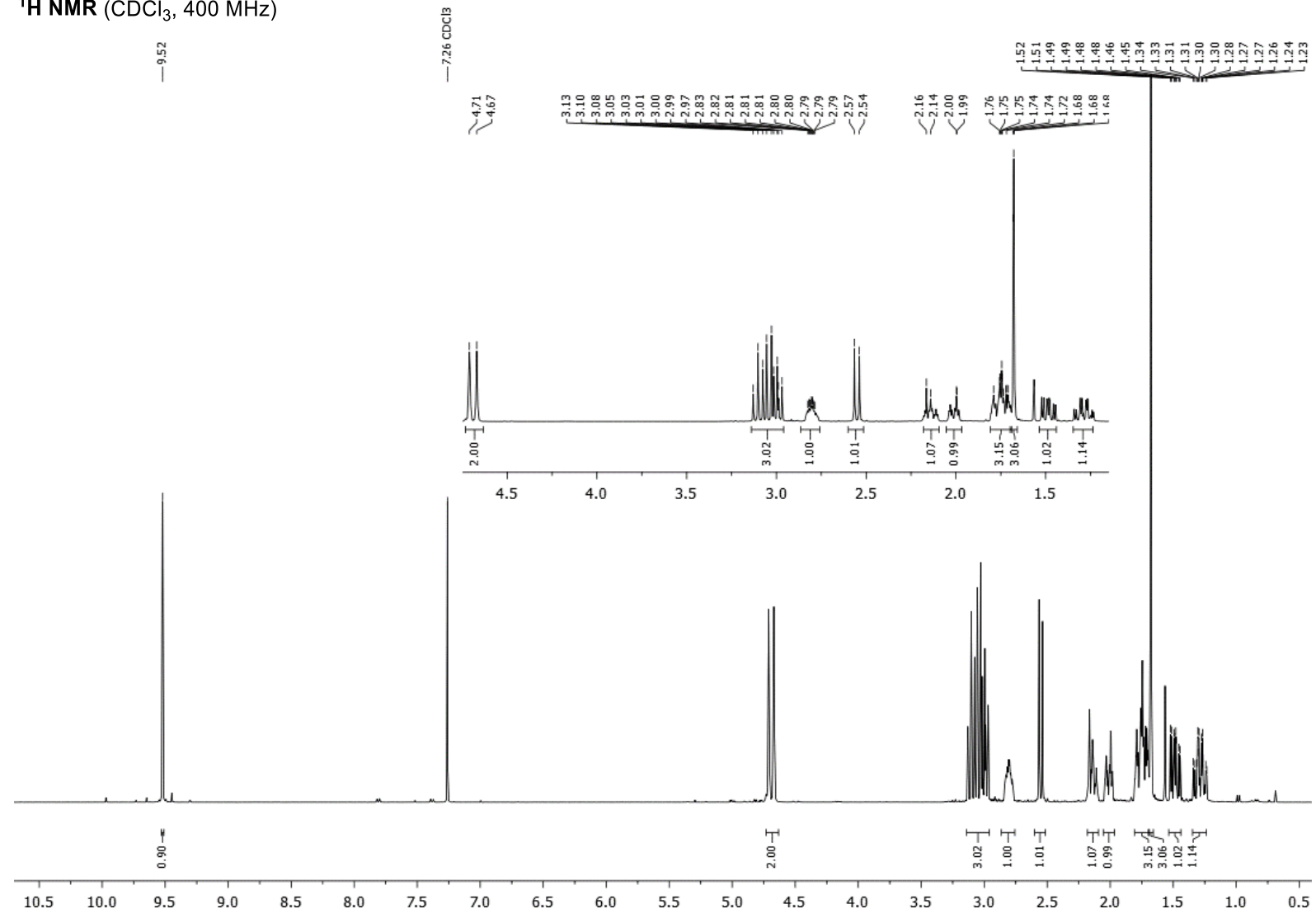

${ }^{13} \mathrm{C}$ NMR $\left(\mathrm{CDCl}_{3}, 101 \mathrm{MHz}\right)$

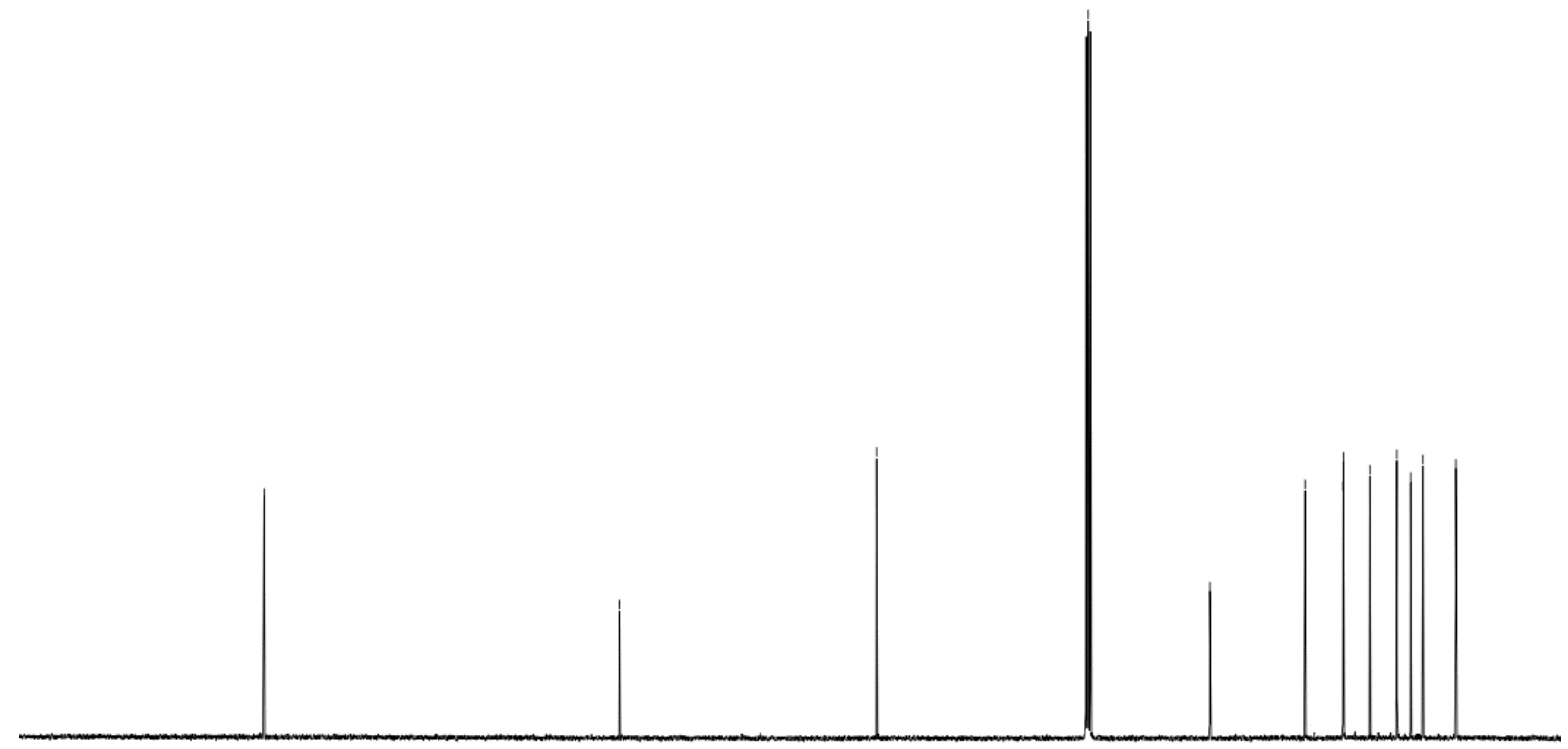

$\begin{array}{llllllllllllllllllllllll}40 & 230 & 220 & 210 & 200 & 190 & 180 & 170 & 160 & 150 & 140 & 130 & 120 & 110 & 100 & 90 & 80 & 70 & 60 & 50 & 40 & 30 & 20 & 10\end{array}$ 


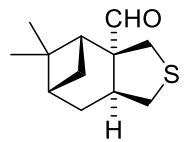

$(-)-8 \mathbf{I}$

${ }^{1} \mathrm{H}$ NMR $\left(\mathrm{CDCl}_{3}, 400 \mathrm{MHz}\right)$

$\stackrel{\substack{0 \\ \hdashline}}{i}$
|

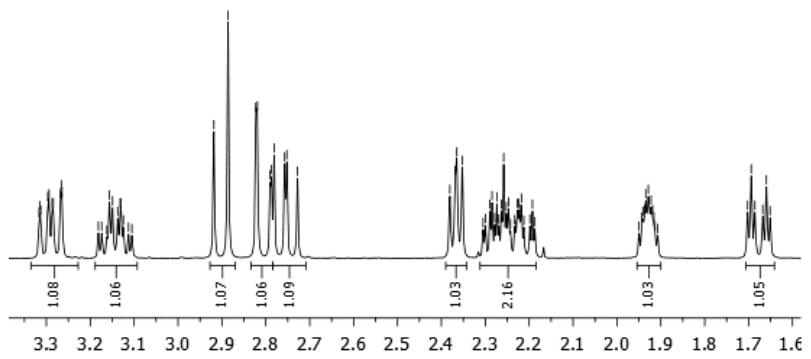

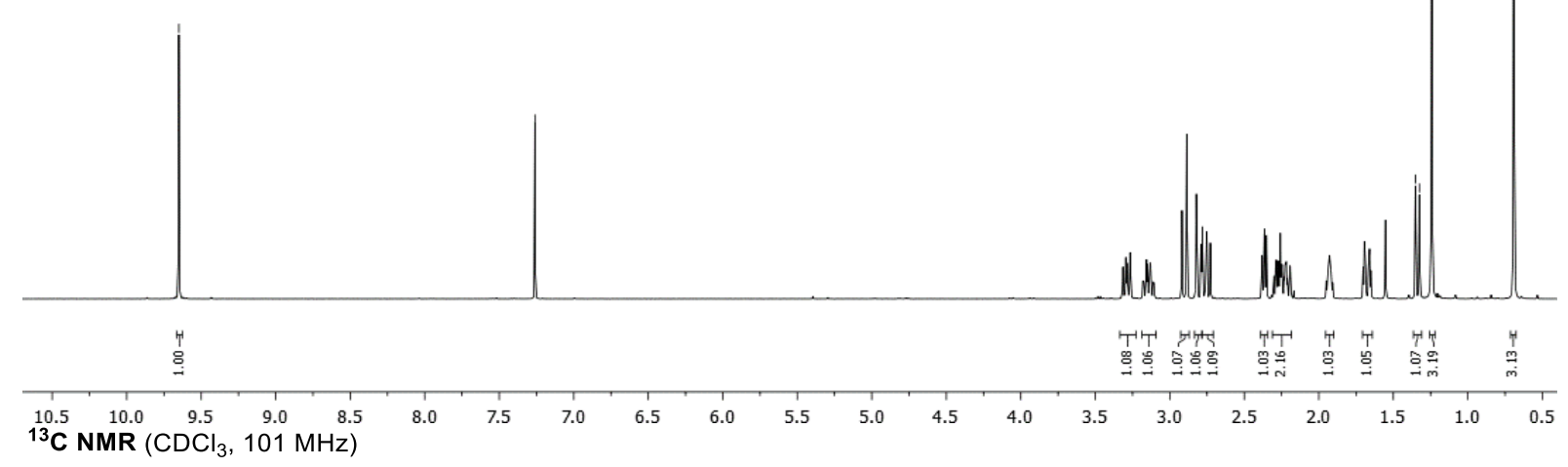

$\vec{i}$
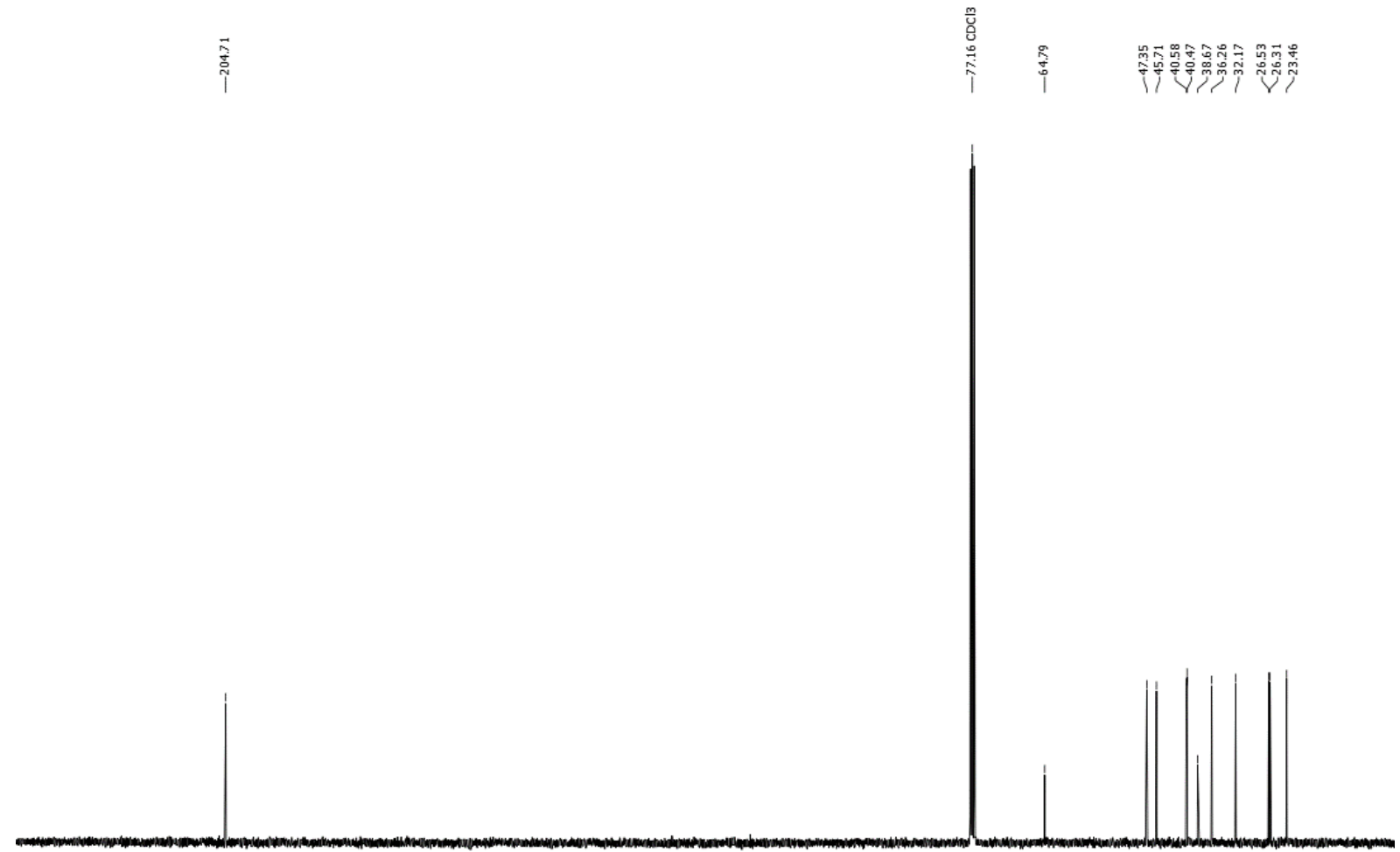

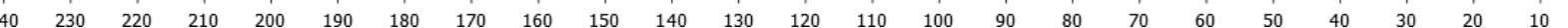




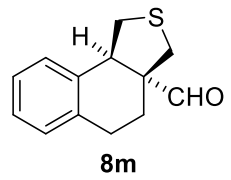

${ }^{1} \mathrm{H}$ NMR $\left(\mathrm{CDCl}_{3}, 400 \mathrm{MHz}\right)$
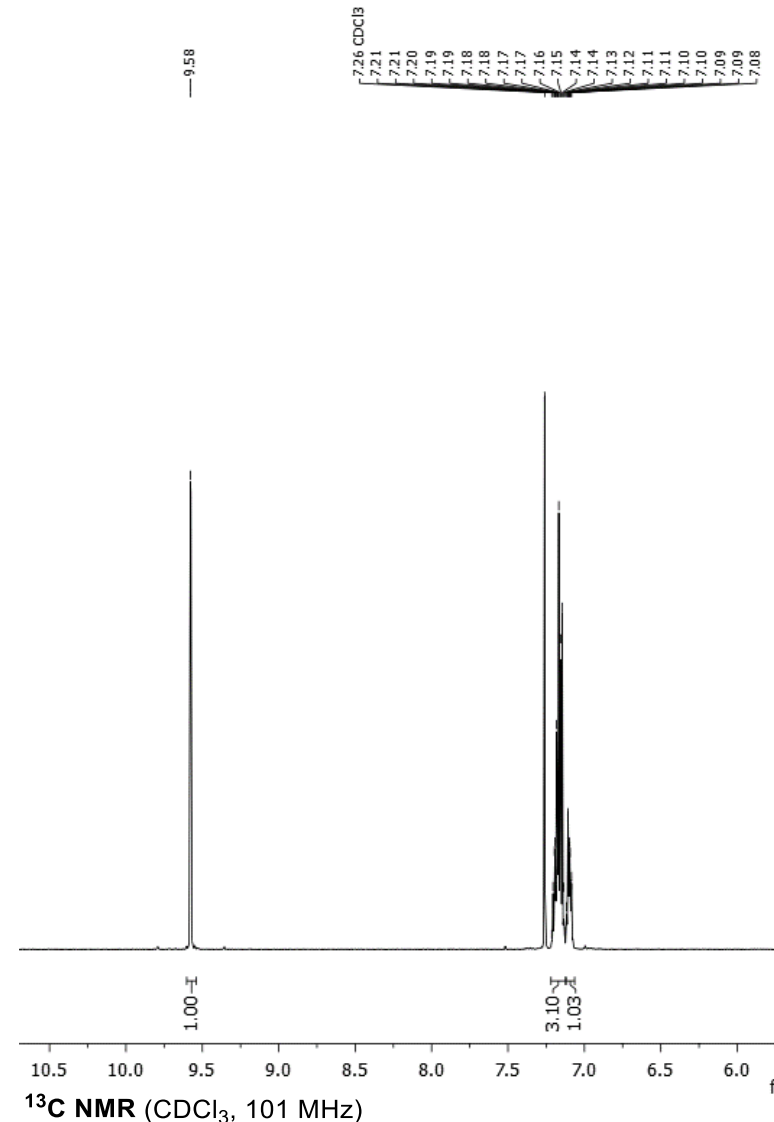

${ }^{13} \mathrm{C} \mathrm{NMR}\left(\mathrm{CDCl}_{3}, 101 \mathrm{MHz}\right)$
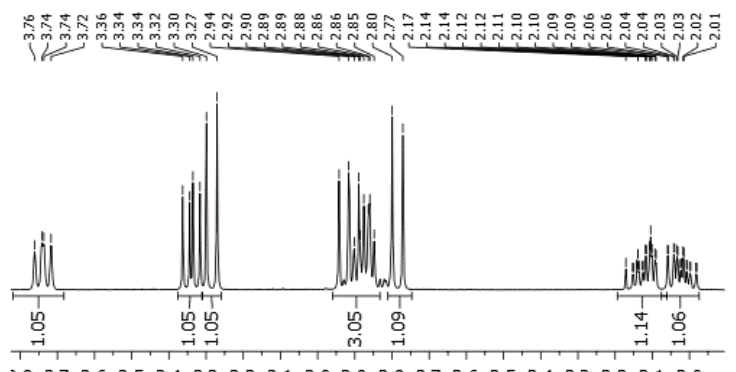

$\begin{array}{lllllllllllllllllll}3.8 & 3.7 & 3.6 & 3.5 & 3.4 & 3.3 & 3.2 & 3.1 & 3.0 & 2.9 & 2.8 & 2.7 & 2.6 & 2.5 & 2.4 & 2.3 & 2.2 & 2.1 & 2.0\end{array}$ f1 (ppm)

$\stackrel{\infty}{\stackrel{\infty}{\oplus}}$

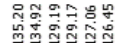

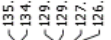

$m$
0
0
0
0

†

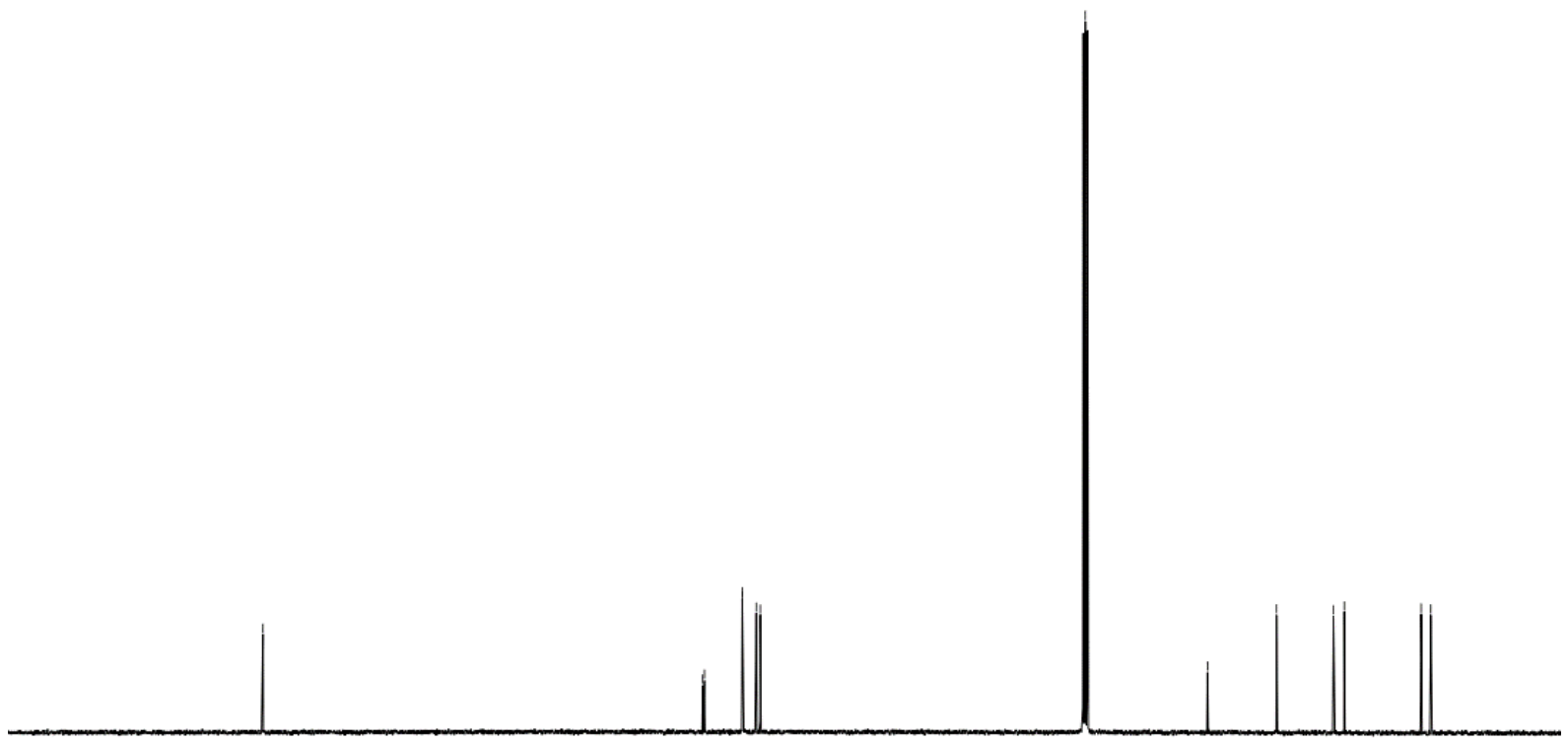

$\begin{array}{llllllllllll}40 & 230 & 220 & 210 & 200 & 190 & 180 & 170 & 160 & 150 & 140 & 130 \begin{array}{r}120 \\ (\mathrm{ppm})\end{array}\end{array}$ 
$\int_{0}^{S} \int_{0}^{2-N}$

$8 n$

${ }^{1} \mathrm{H}$ NMR $\left(\mathrm{CDCl}_{3}, 400 \mathrm{MHz}\right)$

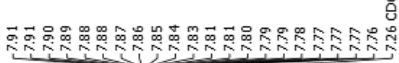

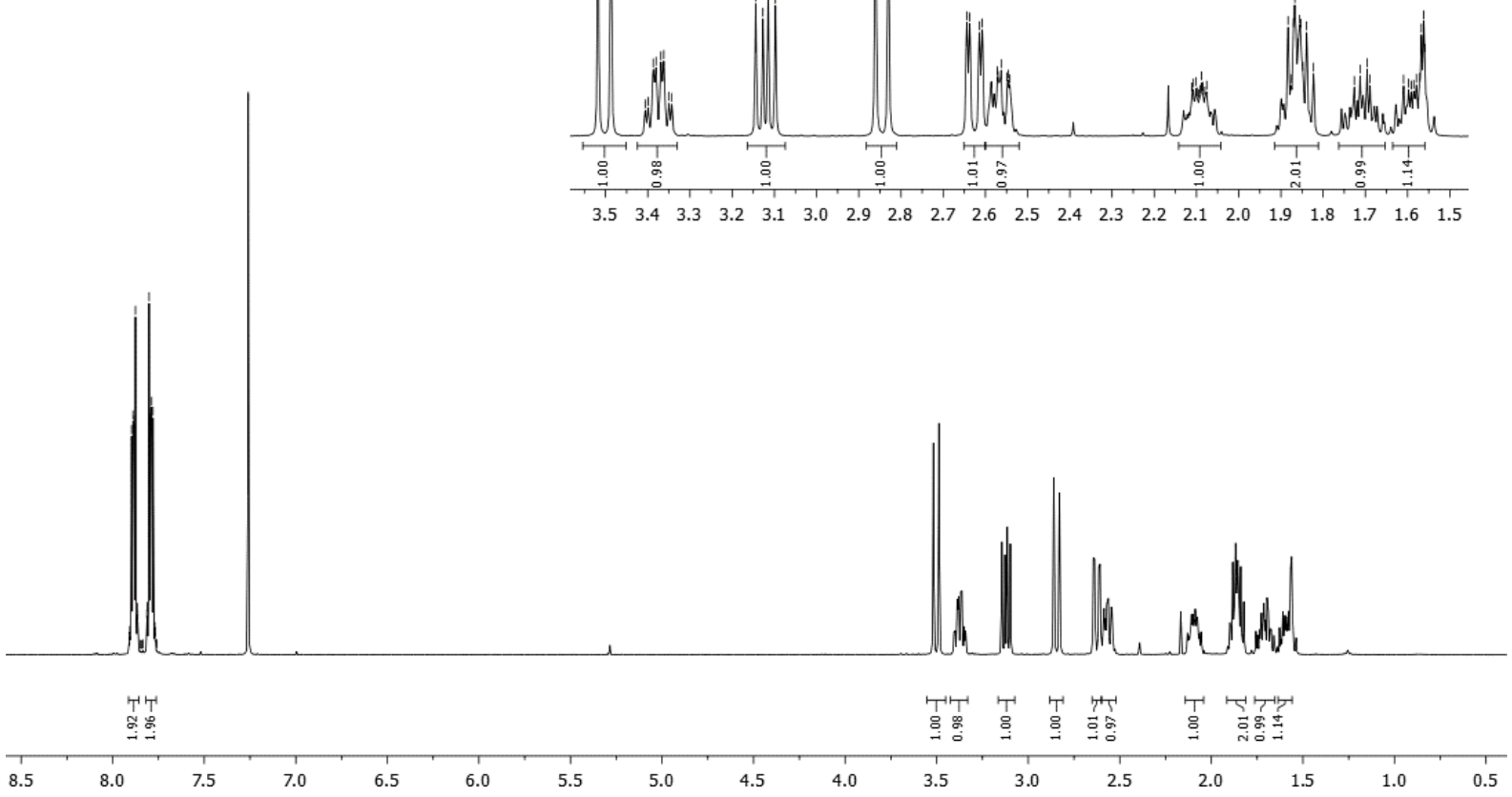

${ }^{13} \mathrm{C}$ NMR $\left(\mathrm{CDCl}_{3}, 101 \mathrm{MHz}\right)$

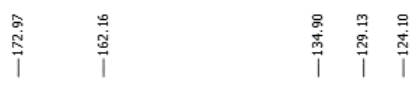

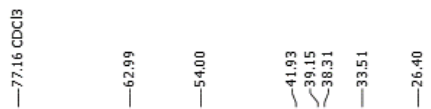

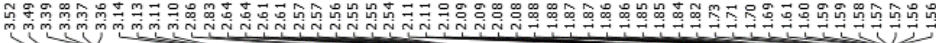

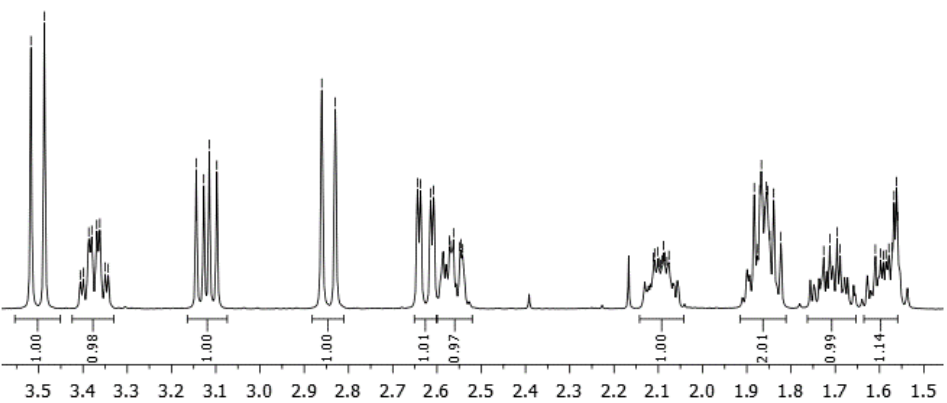

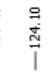

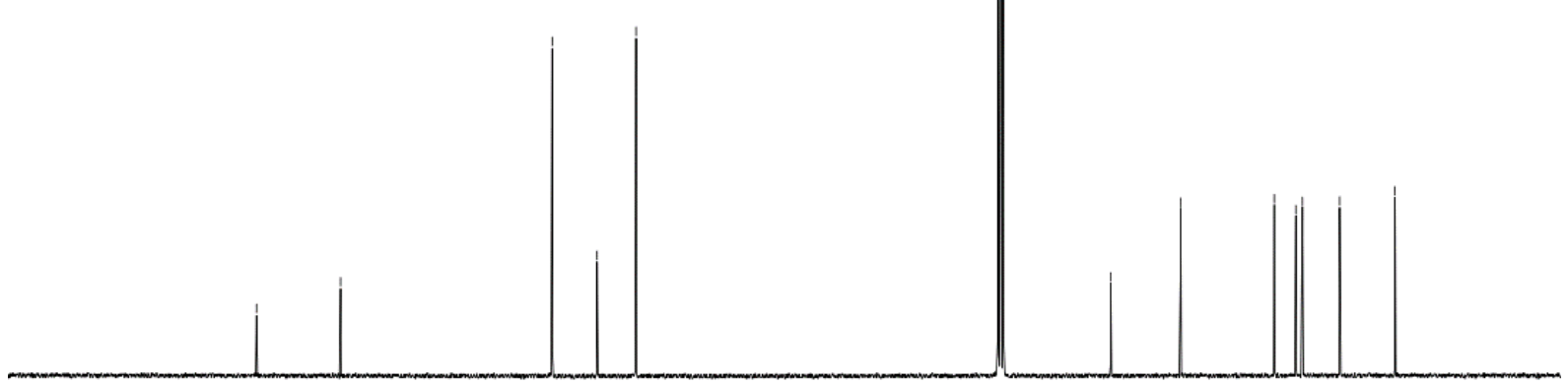

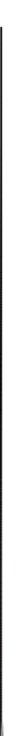




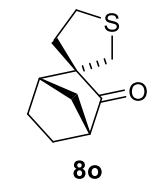

${ }^{1} \mathrm{H}$ NMR $\left(\mathrm{CDCl}_{3}, 400 \mathrm{MHz}\right)$
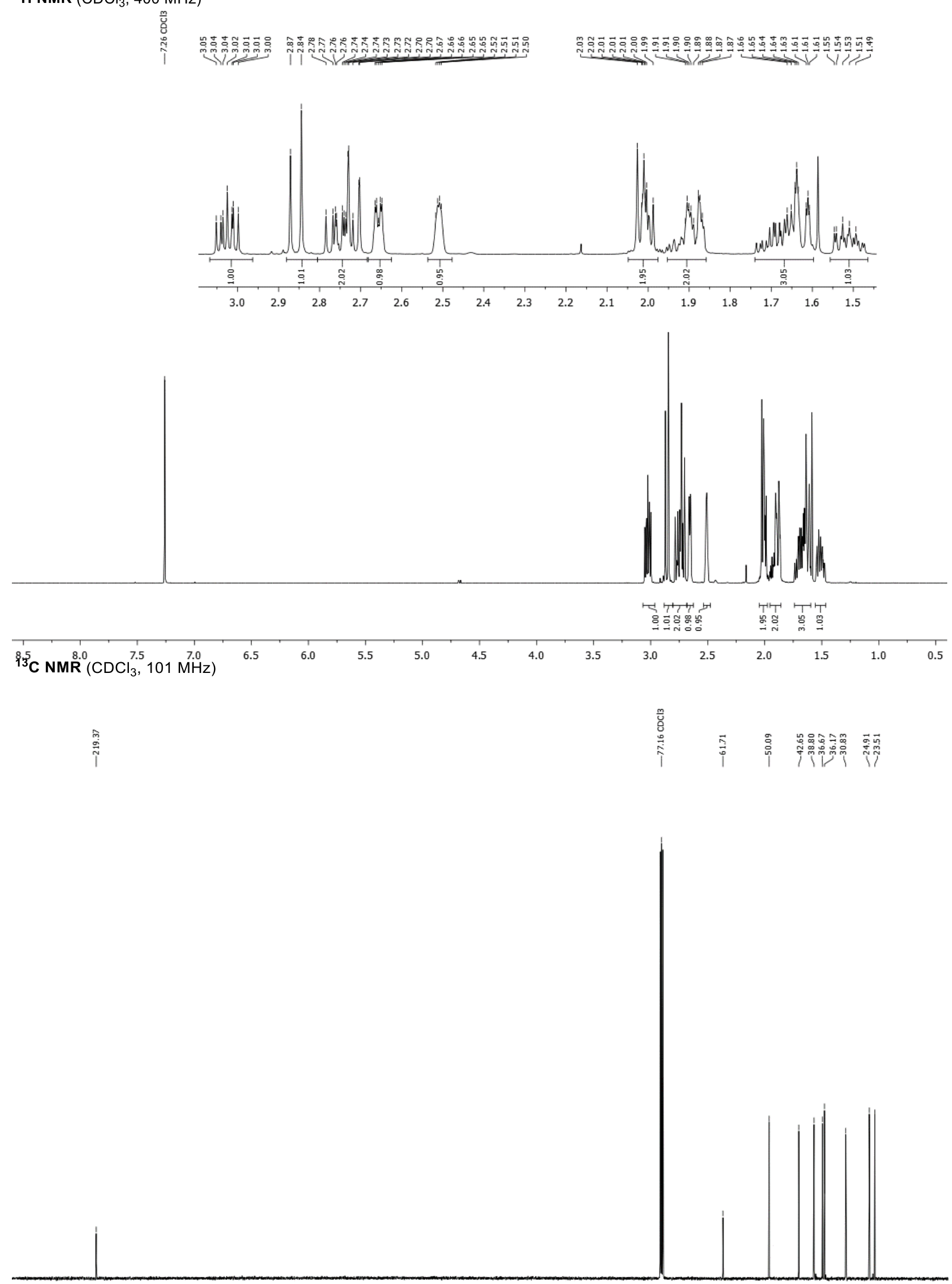

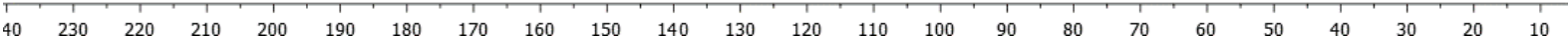




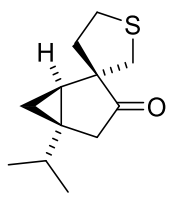

$8 p$

${ }^{1} \mathbf{H}$ NMR $\left(\mathrm{CDCl}_{3}, 400 \mathrm{MHz}\right)$
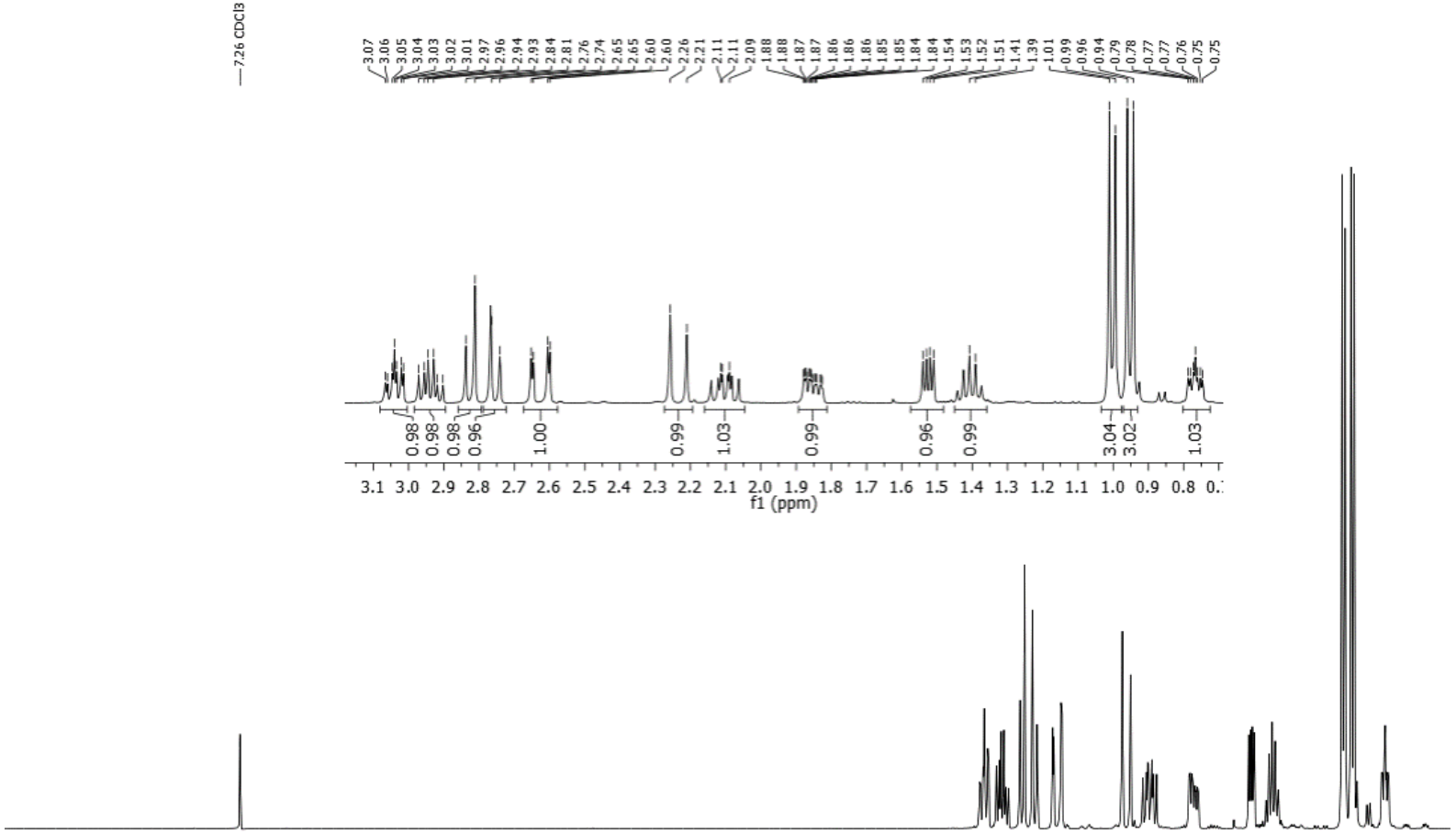

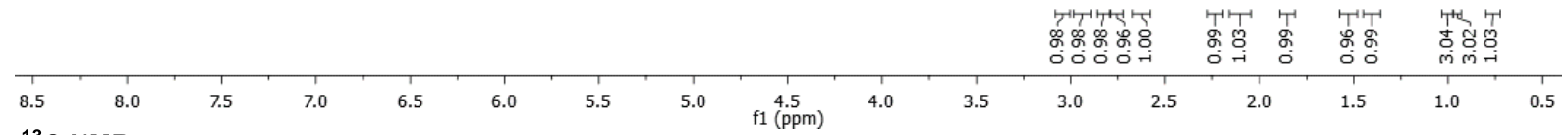

${ }^{13} \mathbf{C}$ NMR $\left(\mathrm{CDCl}_{3}, 101 \mathrm{MHz}\right)$

$\stackrel{g}{\stackrel{g}{\sim}}$
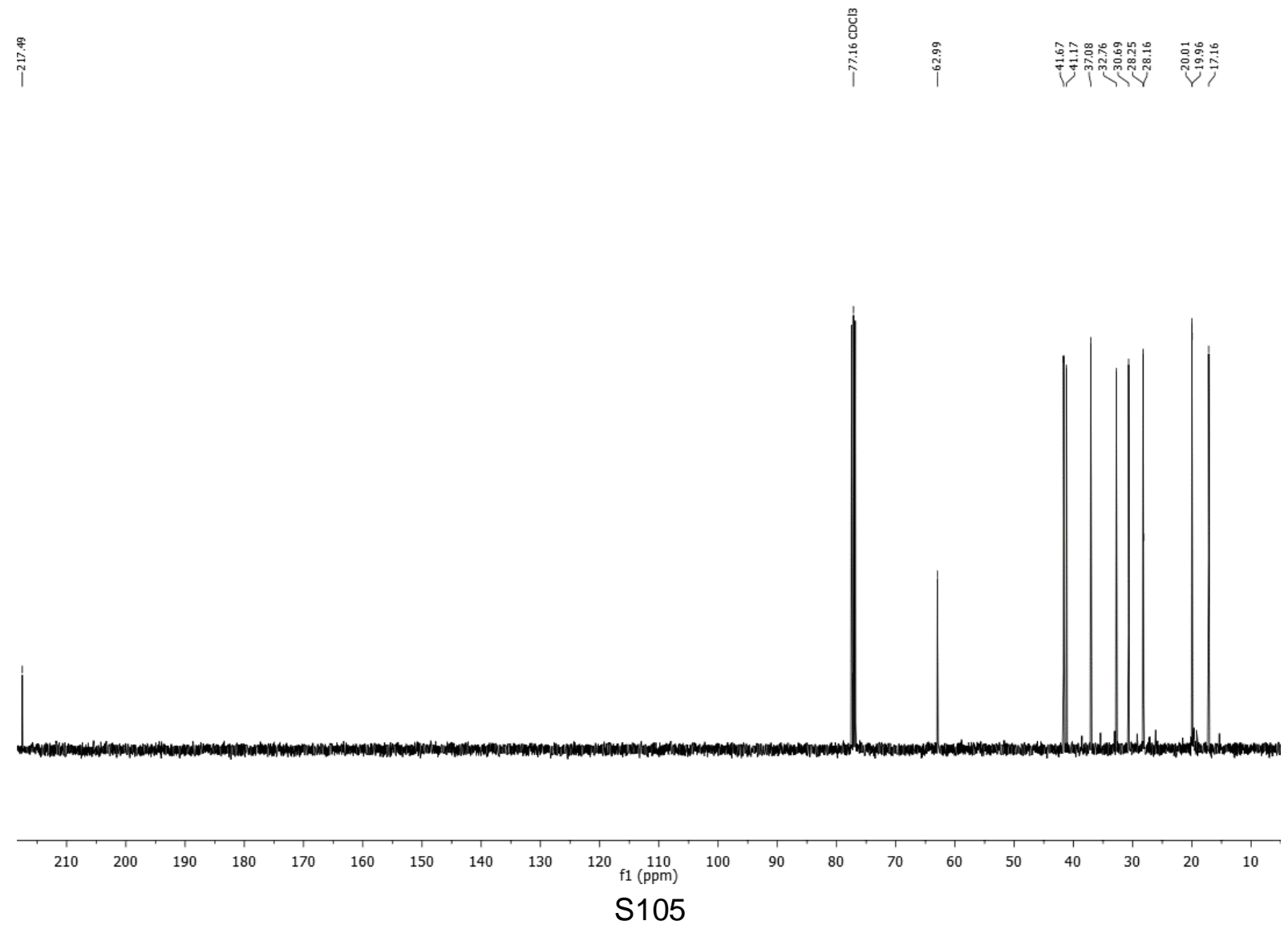


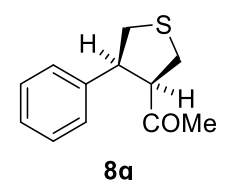

${ }^{1} \mathrm{H}$ NMR $\left(\mathrm{CDCl}_{3}, 400 \mathrm{MHz}\right)$

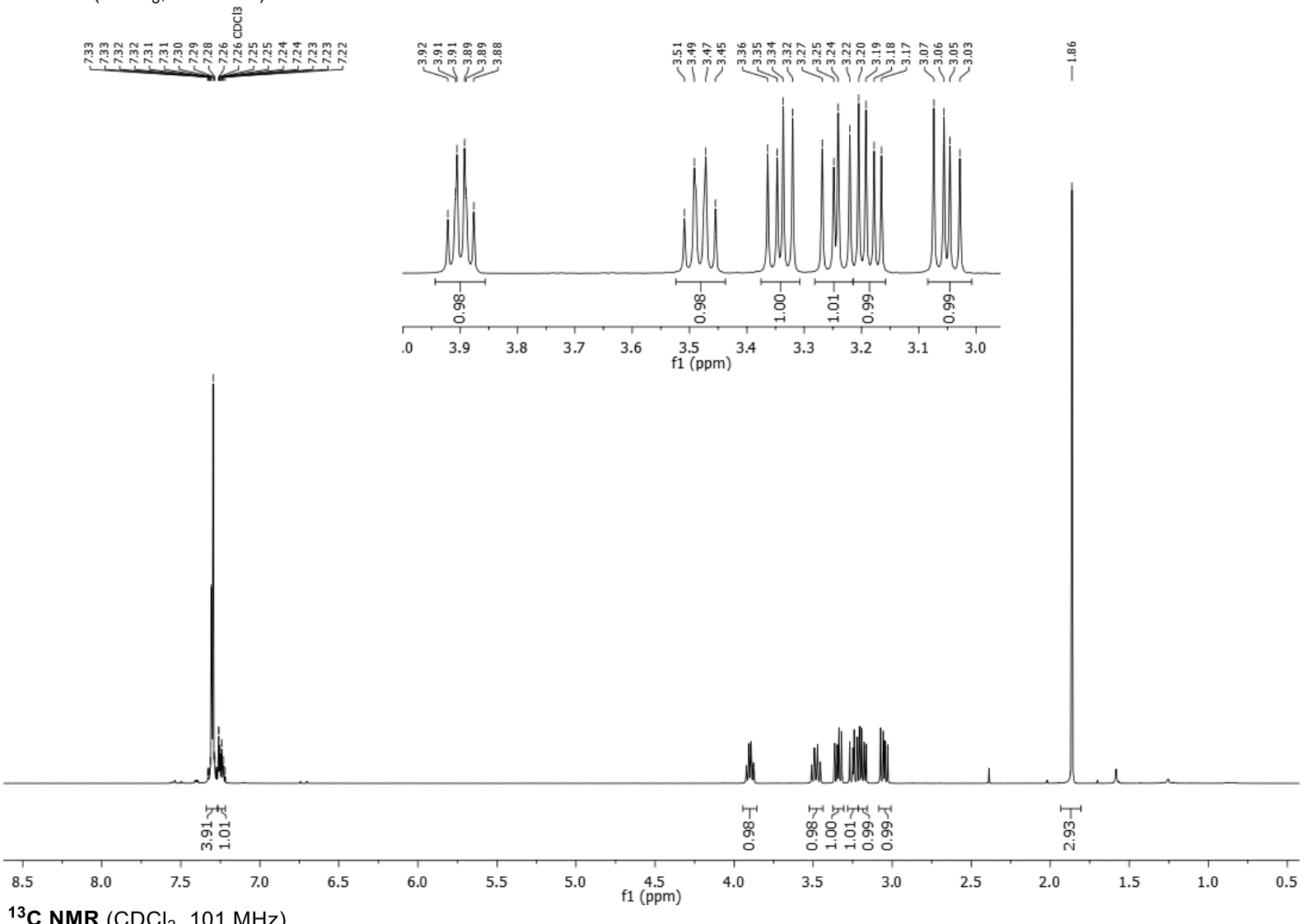

${ }^{13} \mathrm{C} \mathrm{NMR}\left(\mathrm{CDCl}_{3}, 101 \mathrm{MHz}\right)$
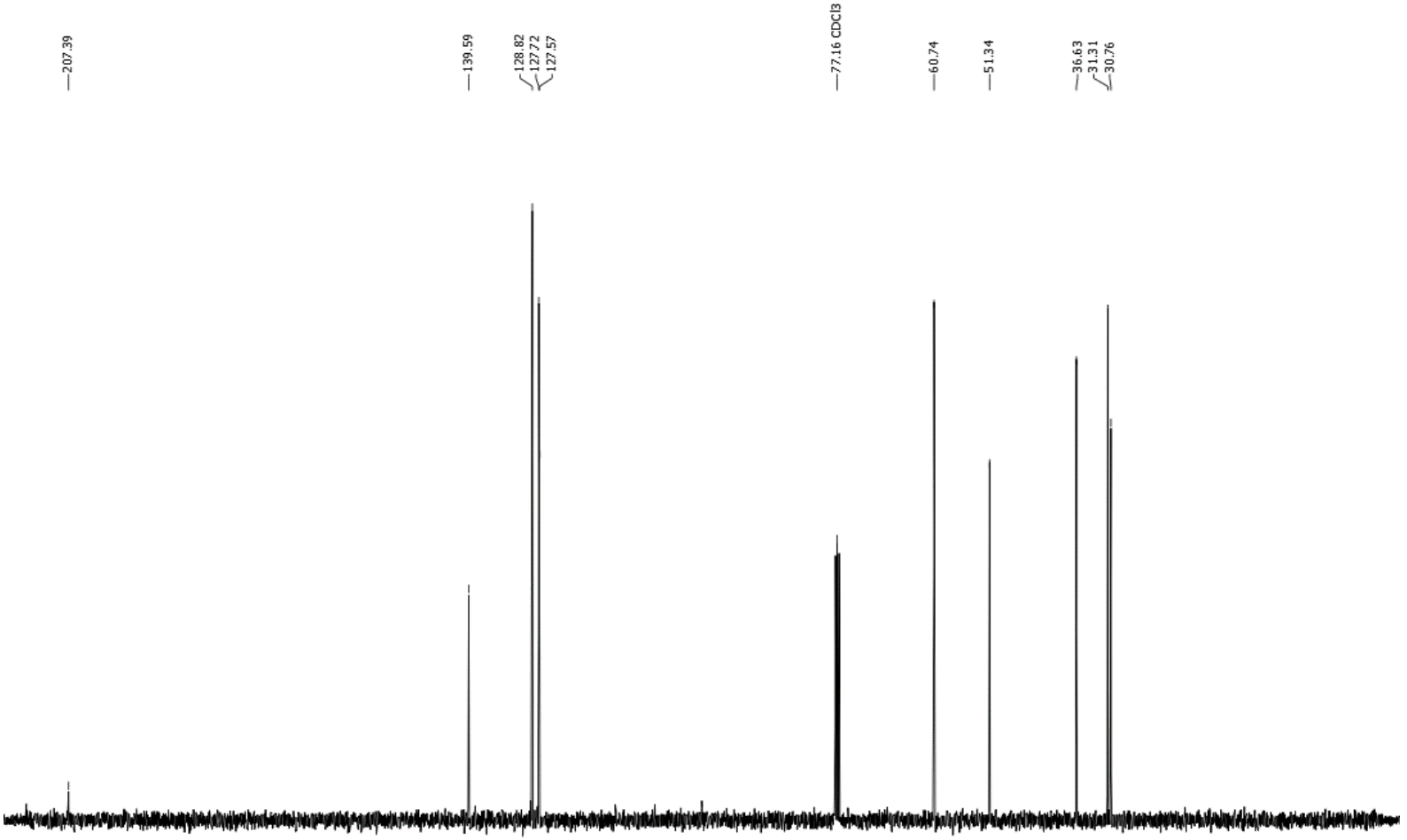

$\begin{array}{lllllllllllllllllllllll}210 & 200 & 190 & 180 & 170 & 160 & 150 & 140 & 130 & 120 & 110 & \begin{array}{c}100 \\ 100\end{array} & 90 & 80 & 70 & 60 & 50 & 40 & 30 & 20 & 10 & 0 & -10\end{array}$ 


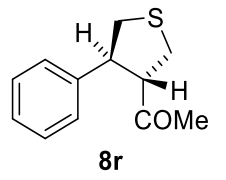

${ }^{1} \mathrm{H} \mathrm{NMR}\left(\mathrm{CDCl}_{3}, 400 \mathrm{MHz}\right)$
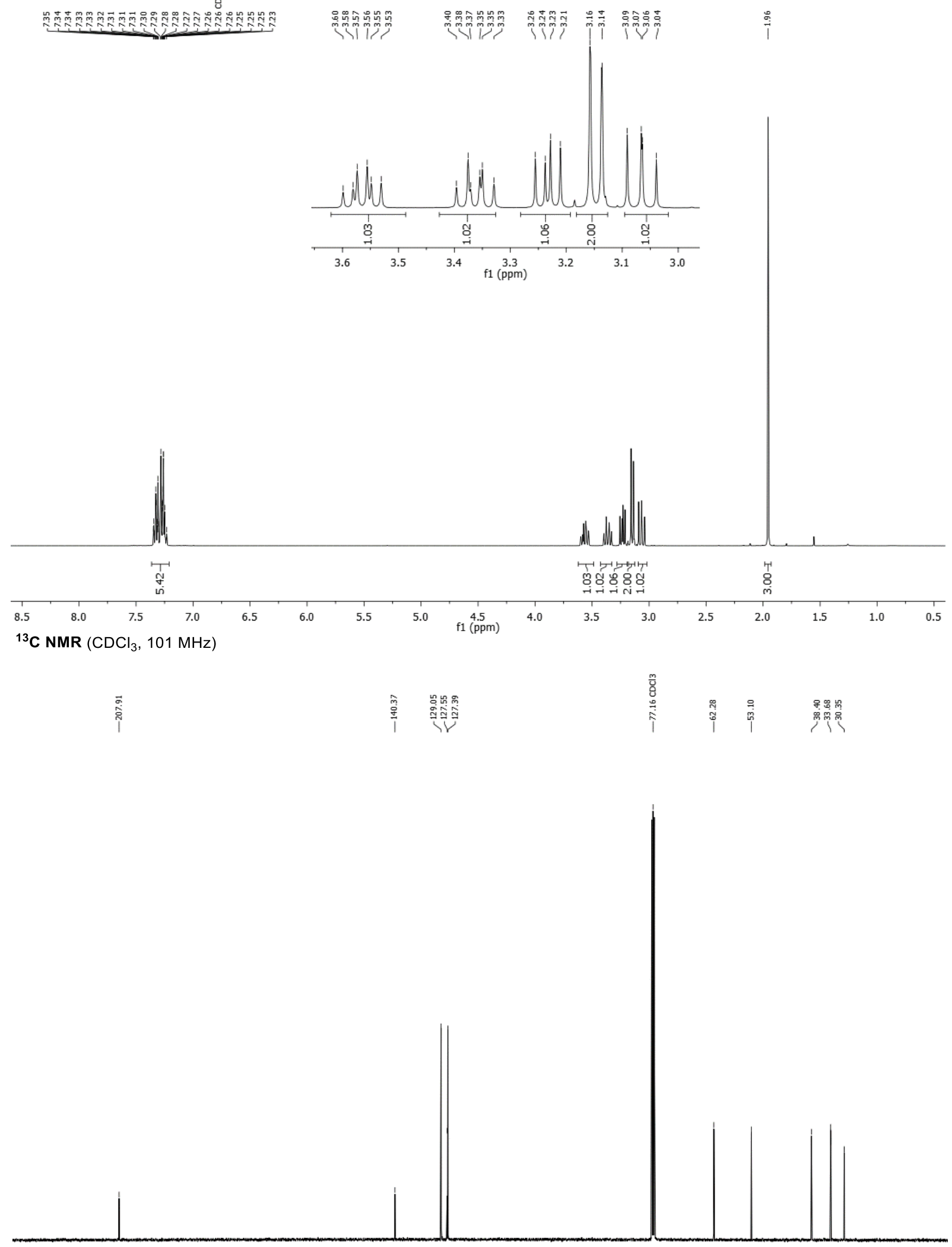

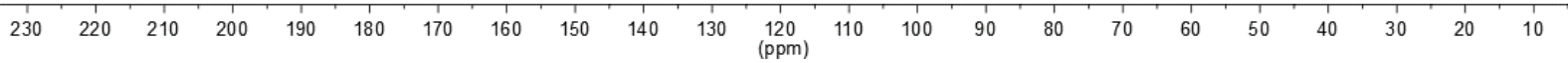




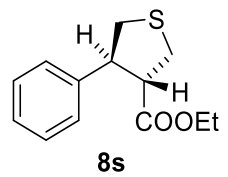

${ }^{1} \mathrm{H}$ NMR $\left(\mathrm{CDCl}_{3}, 599 \mathrm{MHz}\right)$

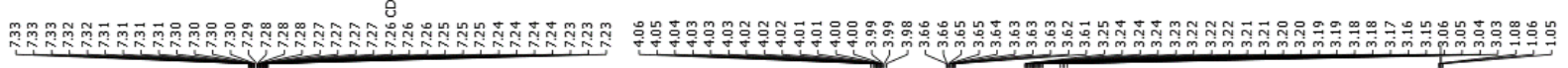

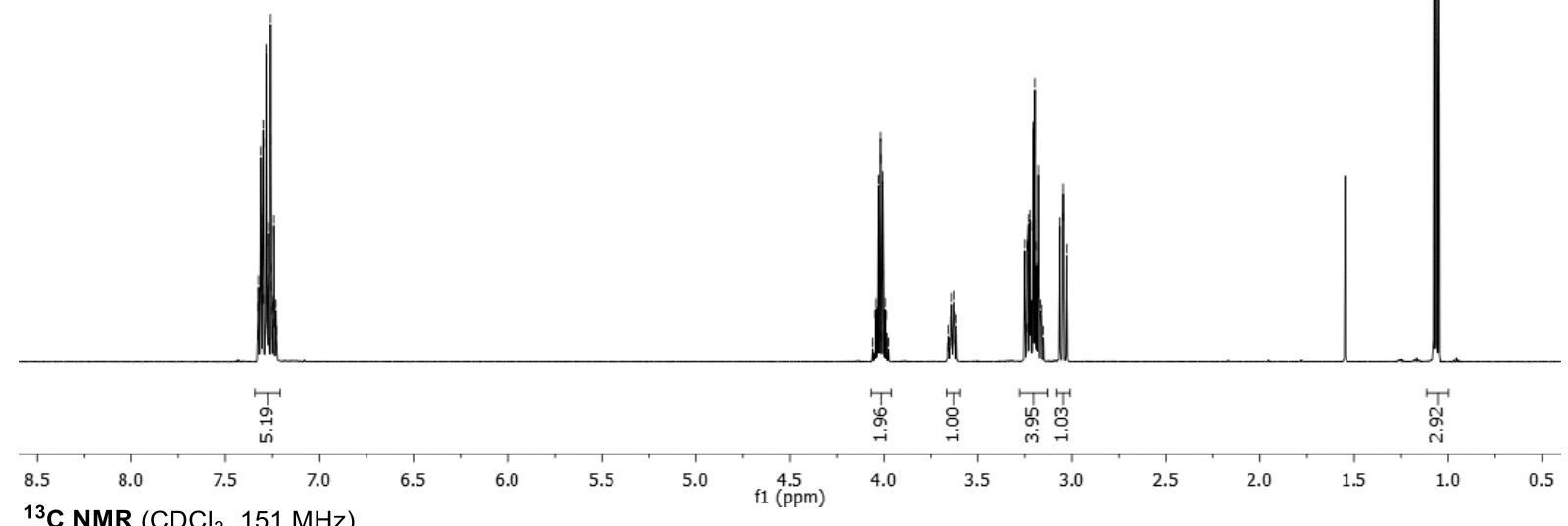

${ }^{13} \mathrm{C} \mathrm{NMR}\left(\mathrm{CDCl}_{3}, 151 \mathrm{MHz}\right)$

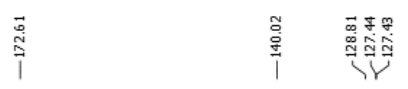

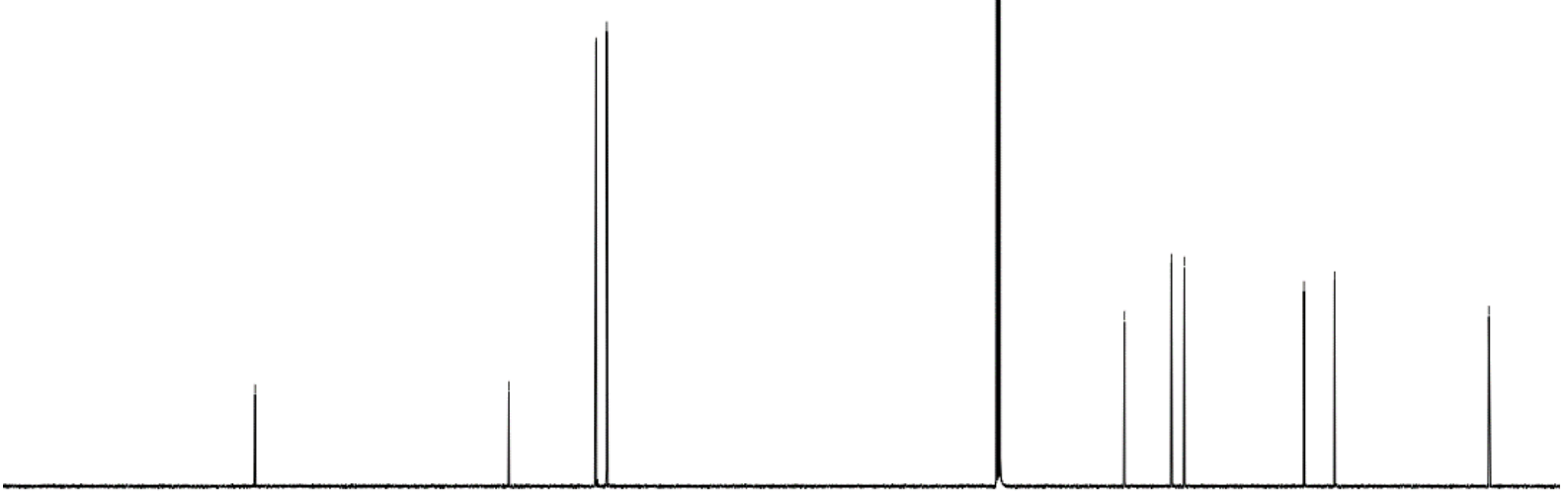




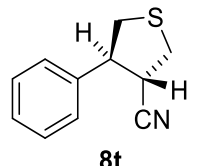

$8 \mathbf{t}$

${ }^{1} \mathrm{H}$ NMR $\left(\mathrm{CDCl}_{3}, 599 \mathrm{MHz}\right)$

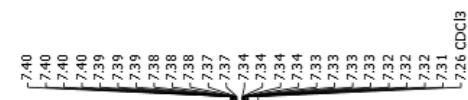
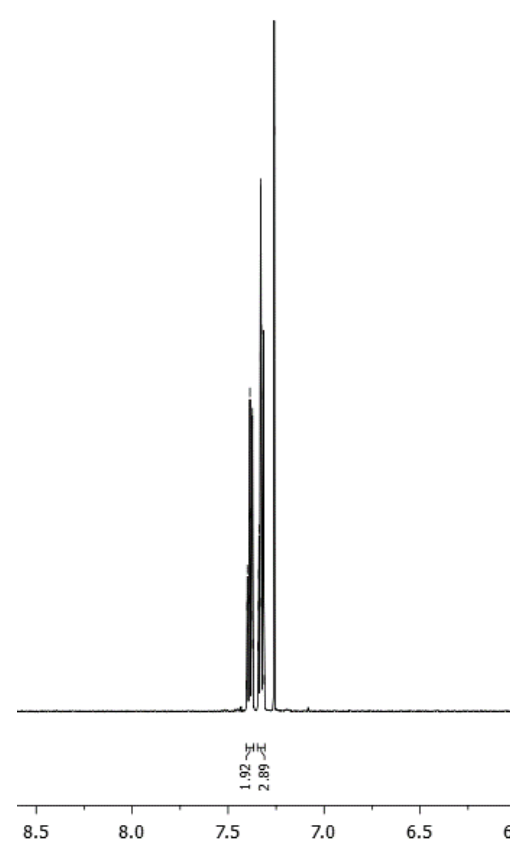

${ }^{13} \mathrm{C}$ NMR $\left(\mathrm{CDCl}_{3}, 151 \mathrm{MHz}\right)$

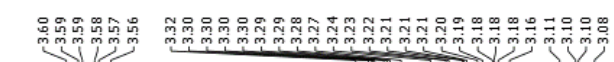
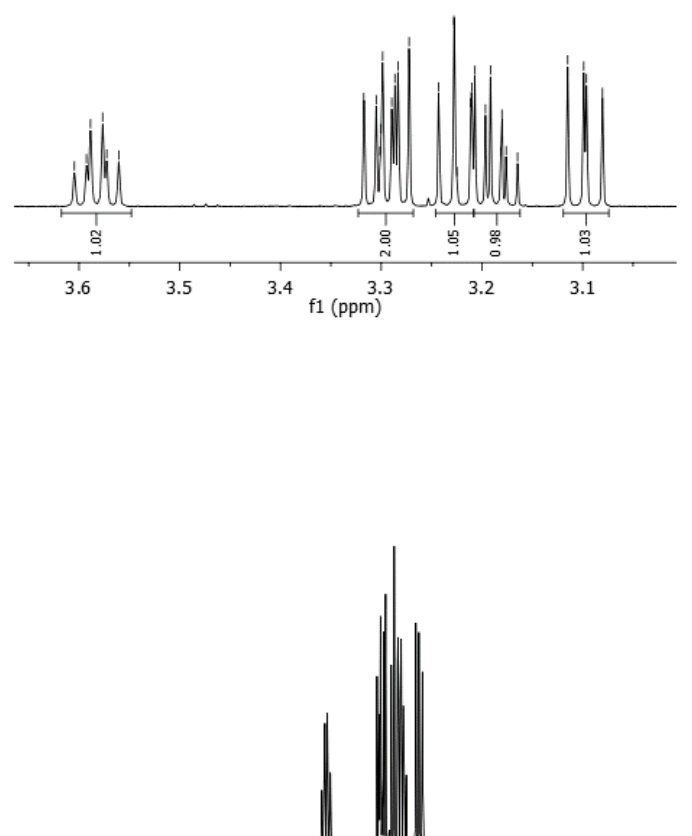

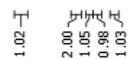
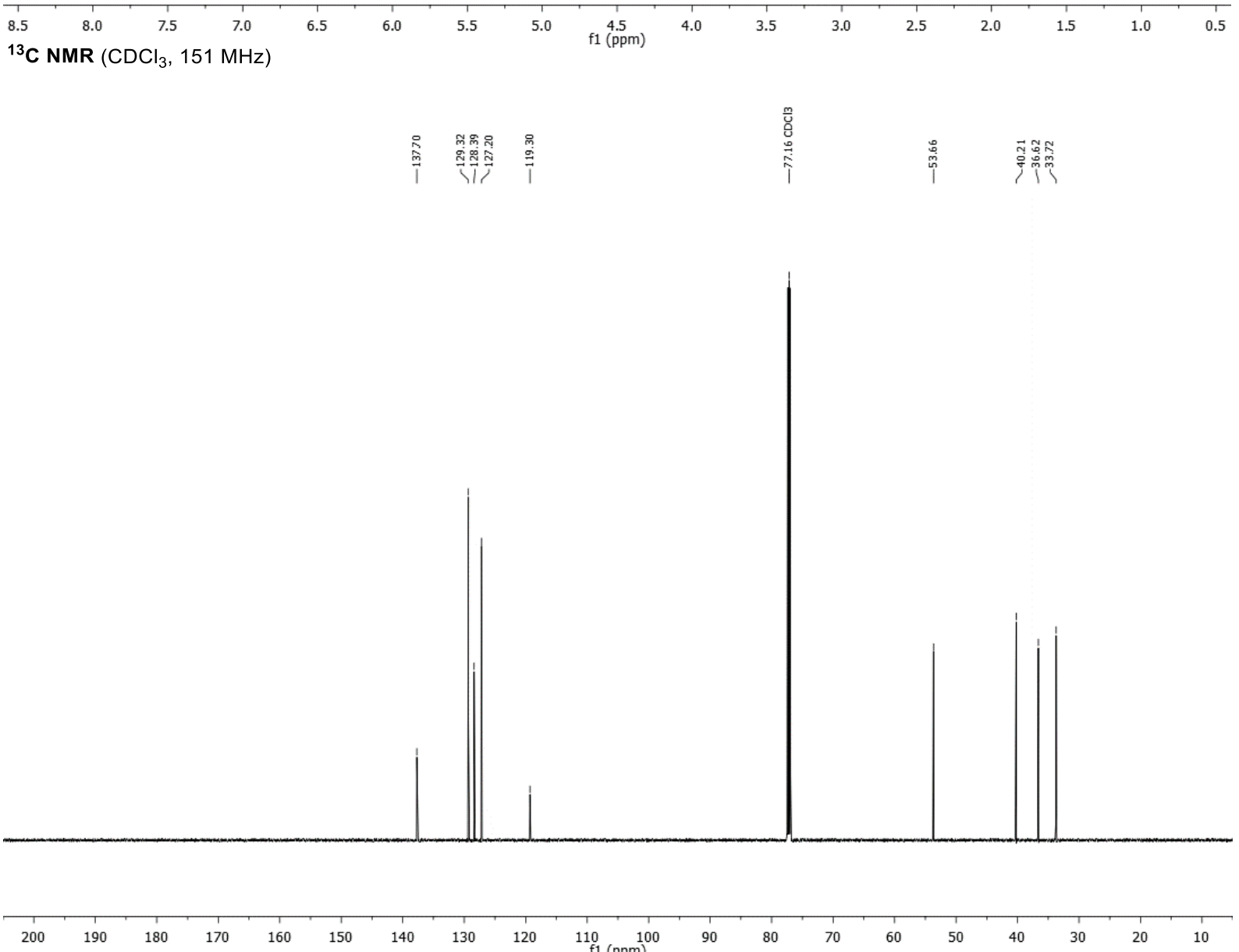

$\underset{\substack{0 \\ \stackrel{0}{0}}}{\stackrel{m}{i}}$

象

180

S109 


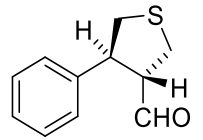

$8 \mathbf{u}$

${ }^{1} \mathrm{H}$ NMR $\left(\mathrm{CDCl}_{3}, 400 \mathrm{MHz}\right)$

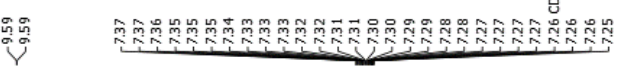

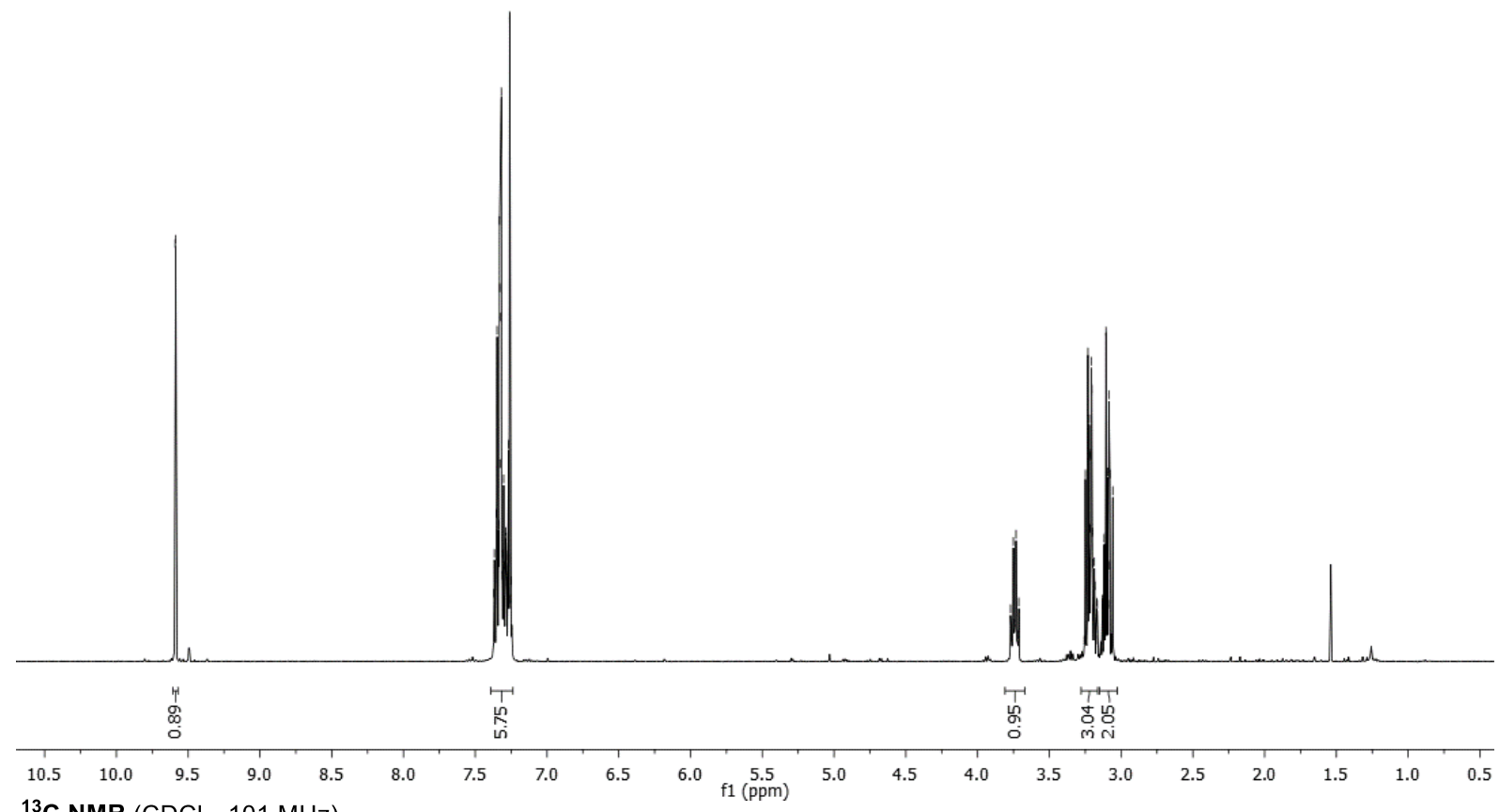

${ }^{13} \mathrm{C}$ NMR $\left(\mathrm{CDCl}_{3}, 101 \mathrm{MHz}\right)$



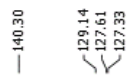

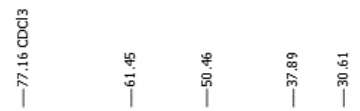

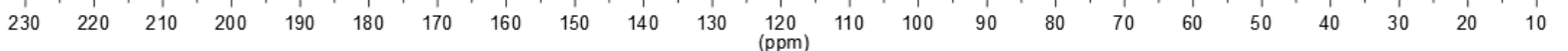




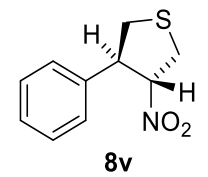

${ }^{1} \mathrm{H}$ NMR $\left(\mathrm{CDCl}_{3}, 599 \mathrm{MHz}\right)$

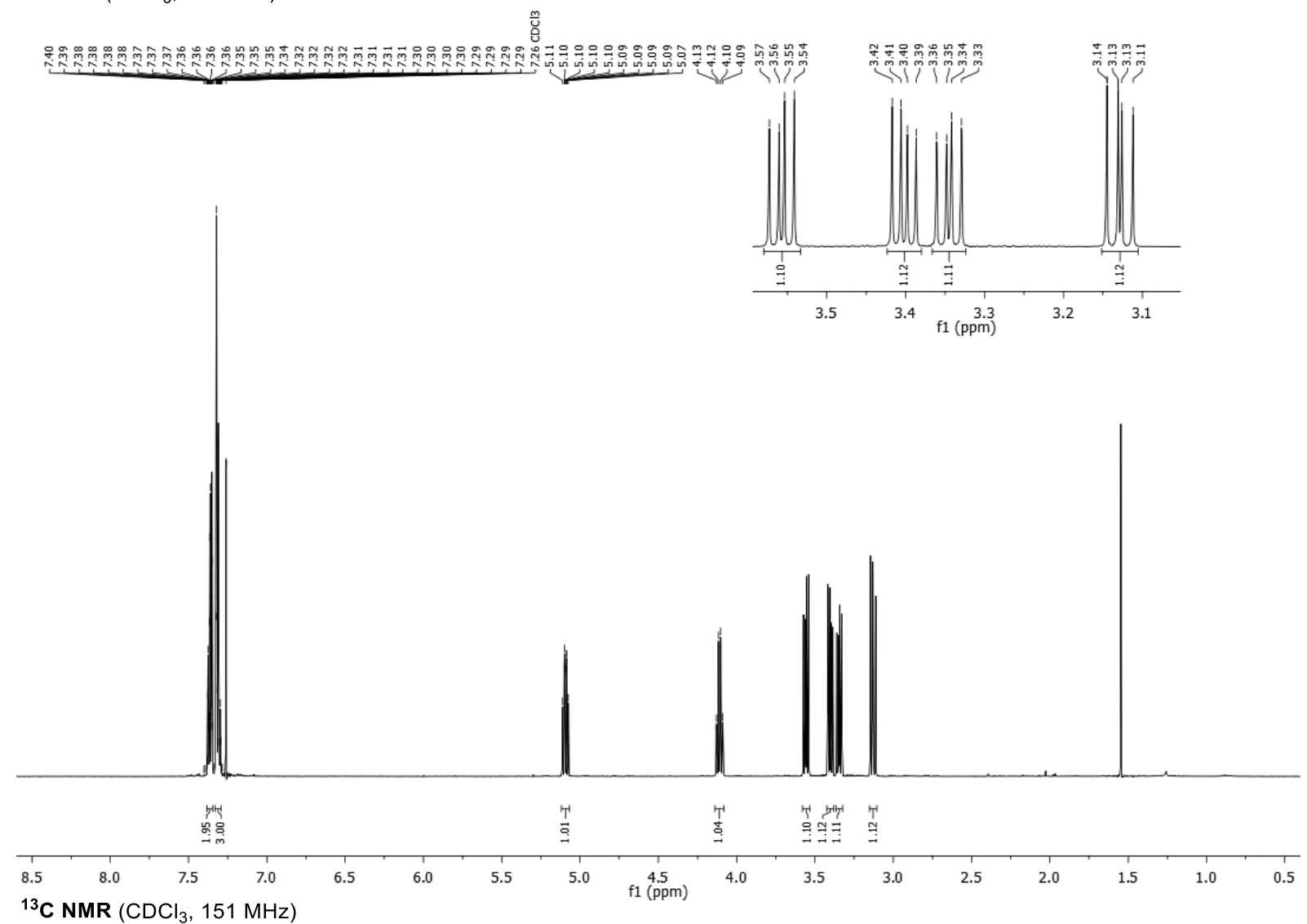

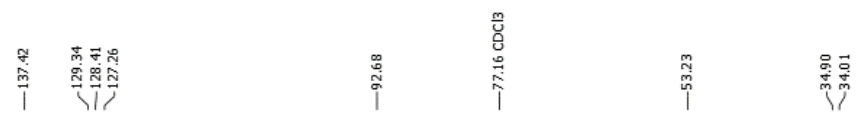

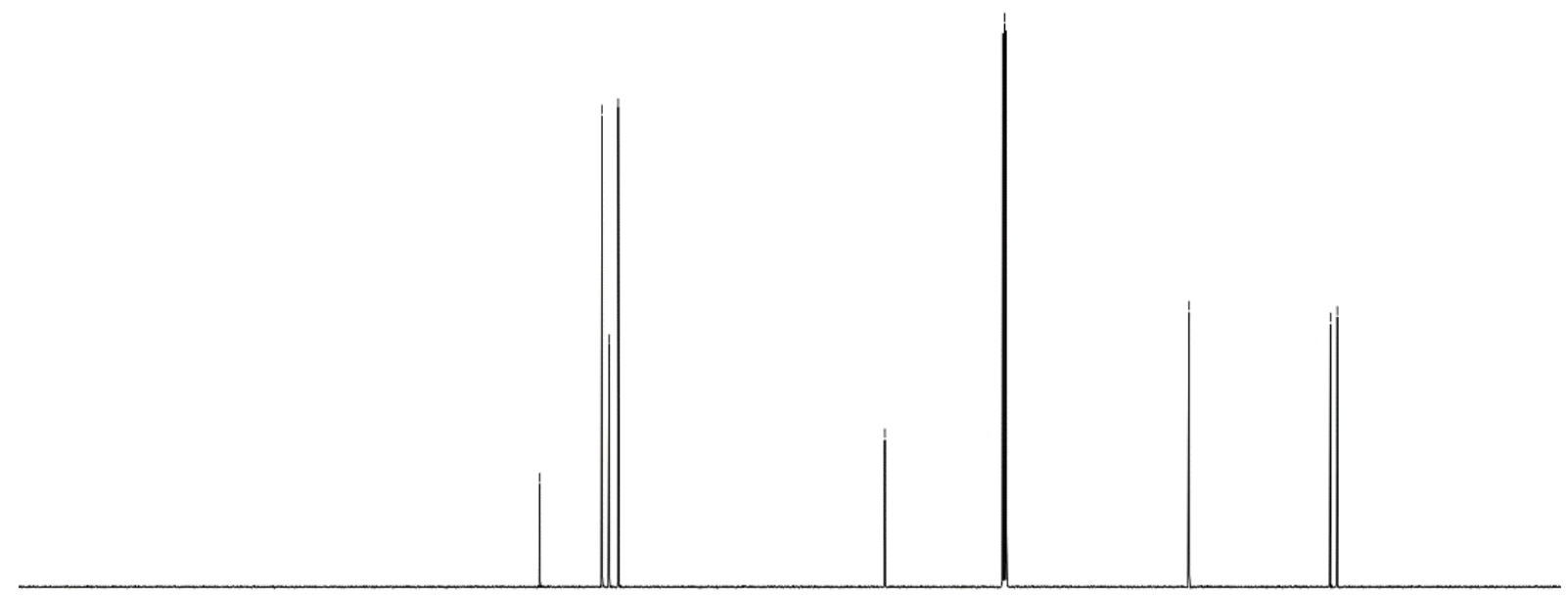


<smiles>CC1(C=O)CSCC1c1ccccc1</smiles>

$8 \mathrm{w}$

${ }^{1} \mathrm{H} \mathrm{NMR}\left(\mathrm{CDCl}_{3}, 400 \mathrm{MHz}\right)$

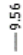

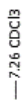

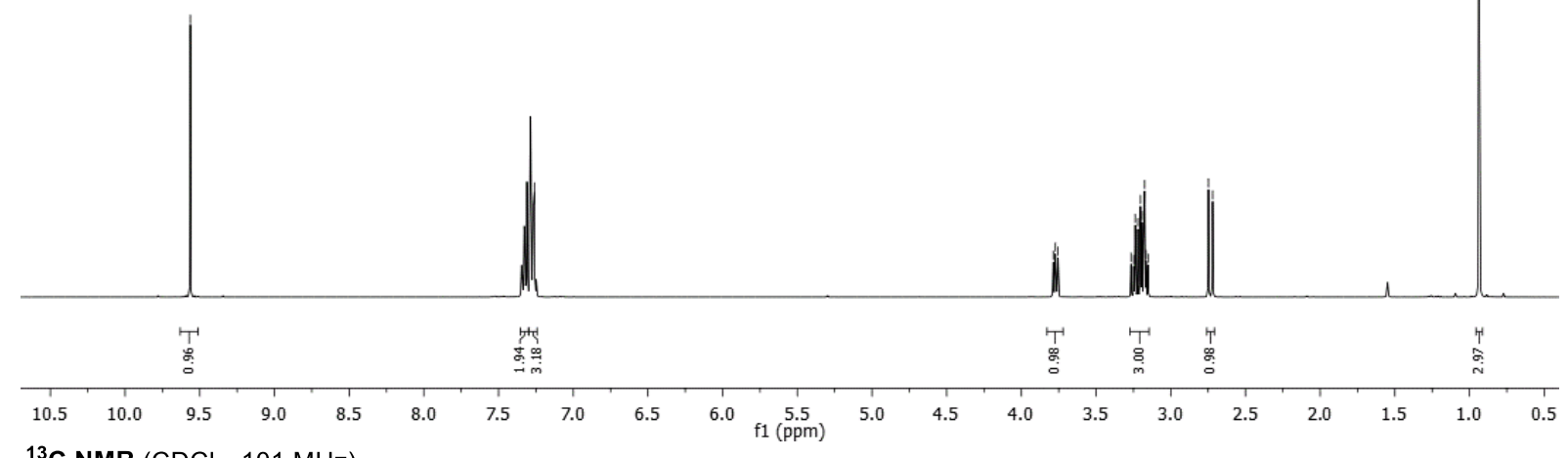

${ }^{13} \mathrm{C} \mathrm{NMR}\left(\mathrm{CDCl}_{3}, 101 \mathrm{MHz}\right)$
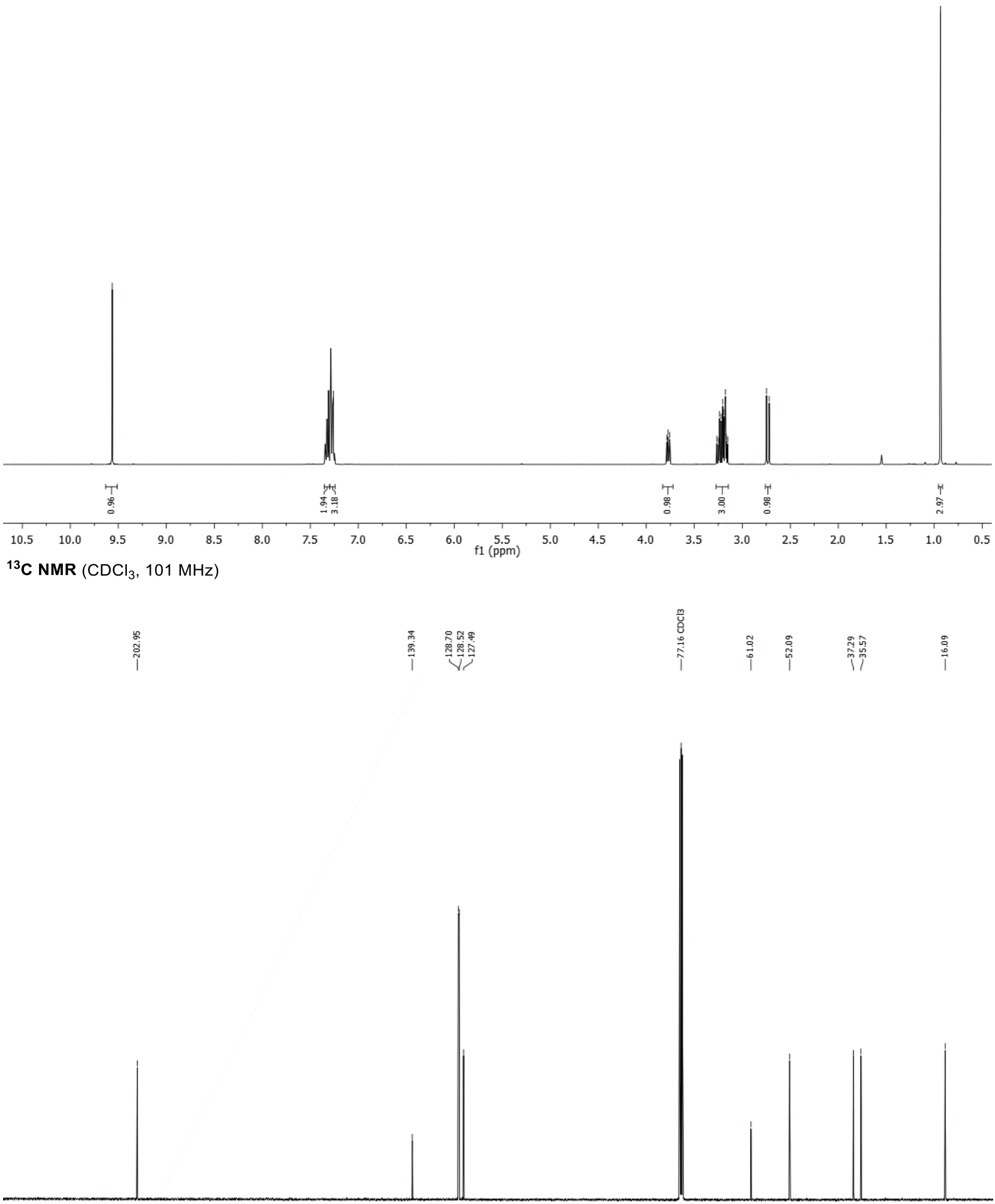


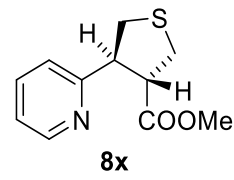

${ }^{1} \mathrm{H} \mathrm{NMR}\left(\mathrm{CDCl}_{3}, 400 \mathrm{MHz}\right)$

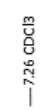

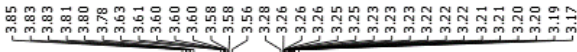

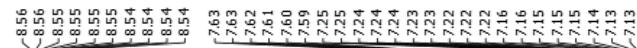
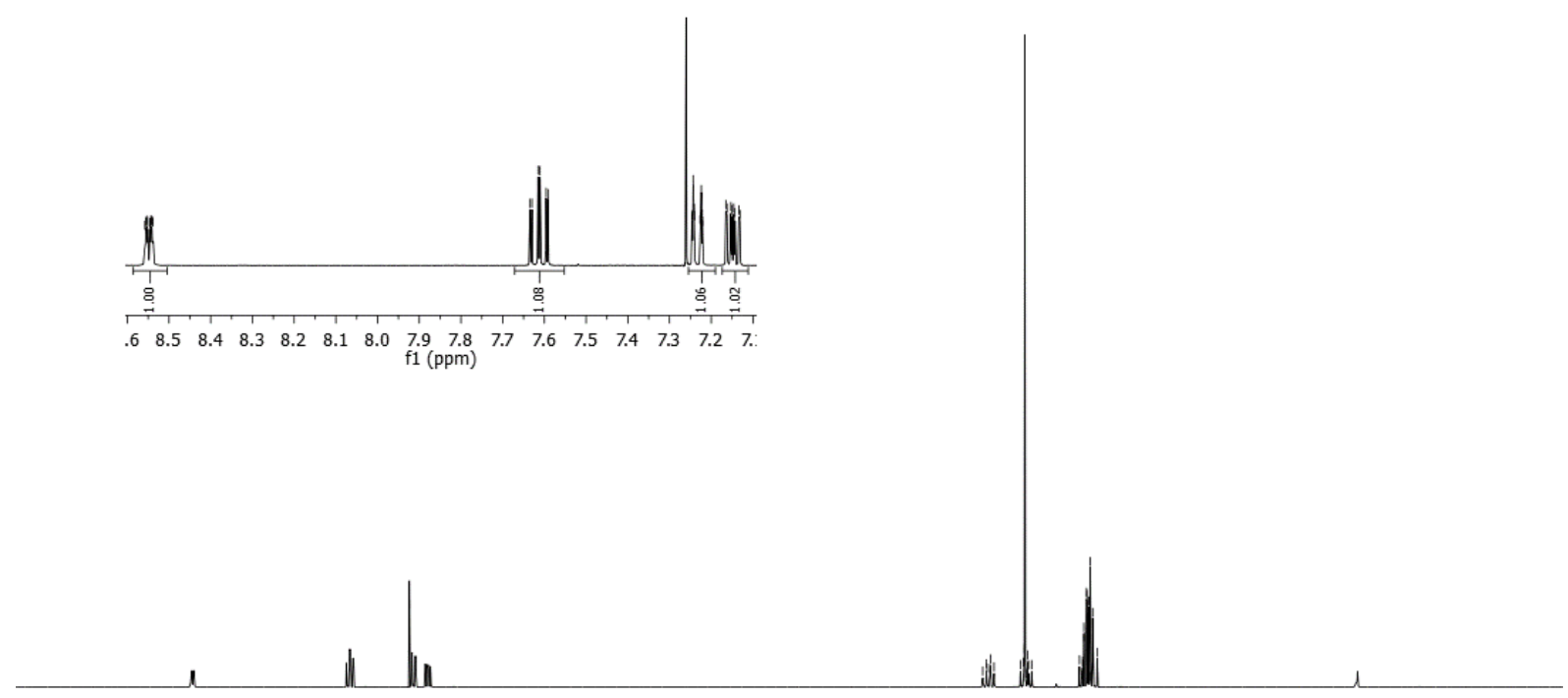

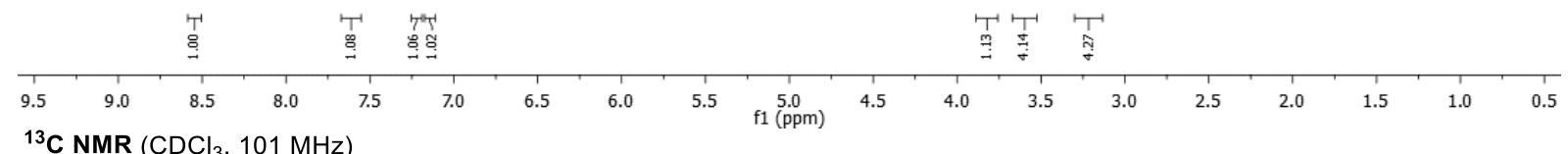

${ }^{13} \mathrm{C} \mathrm{NMR}\left(\mathrm{CDCl}_{3}, 101 \mathrm{MHz}\right)$

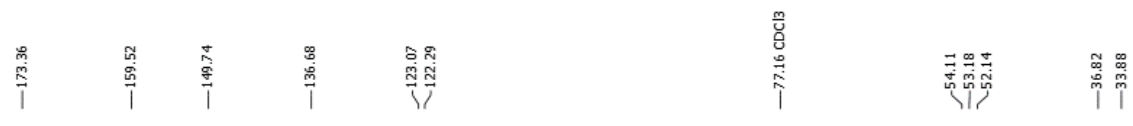

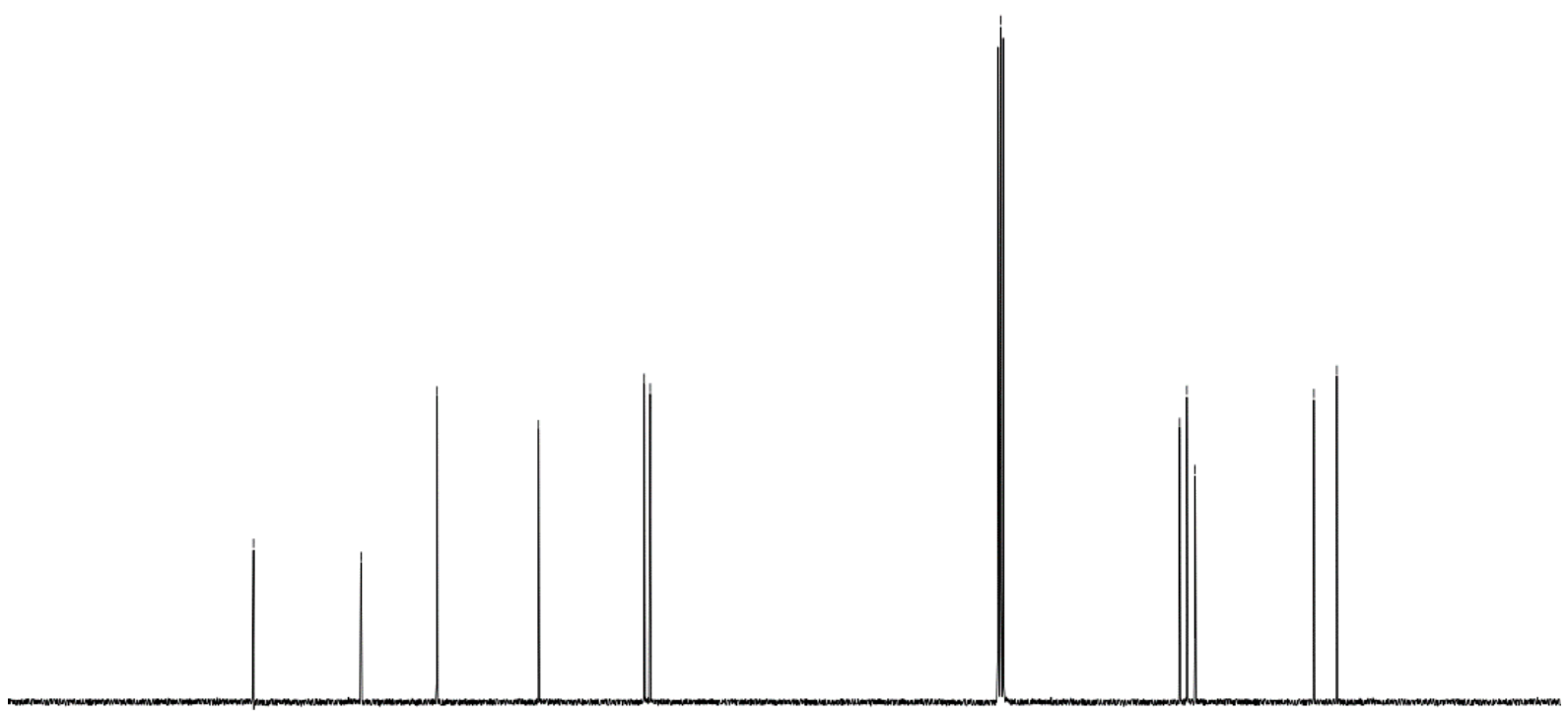

$\begin{array}{llllllllll}200 & 190 & 180 & 170 & 160 & 150 & 140 & 130 & 120 & 110 \\ \mathrm{f} 1(\mathrm{ppm}) & 100\end{array}$ 


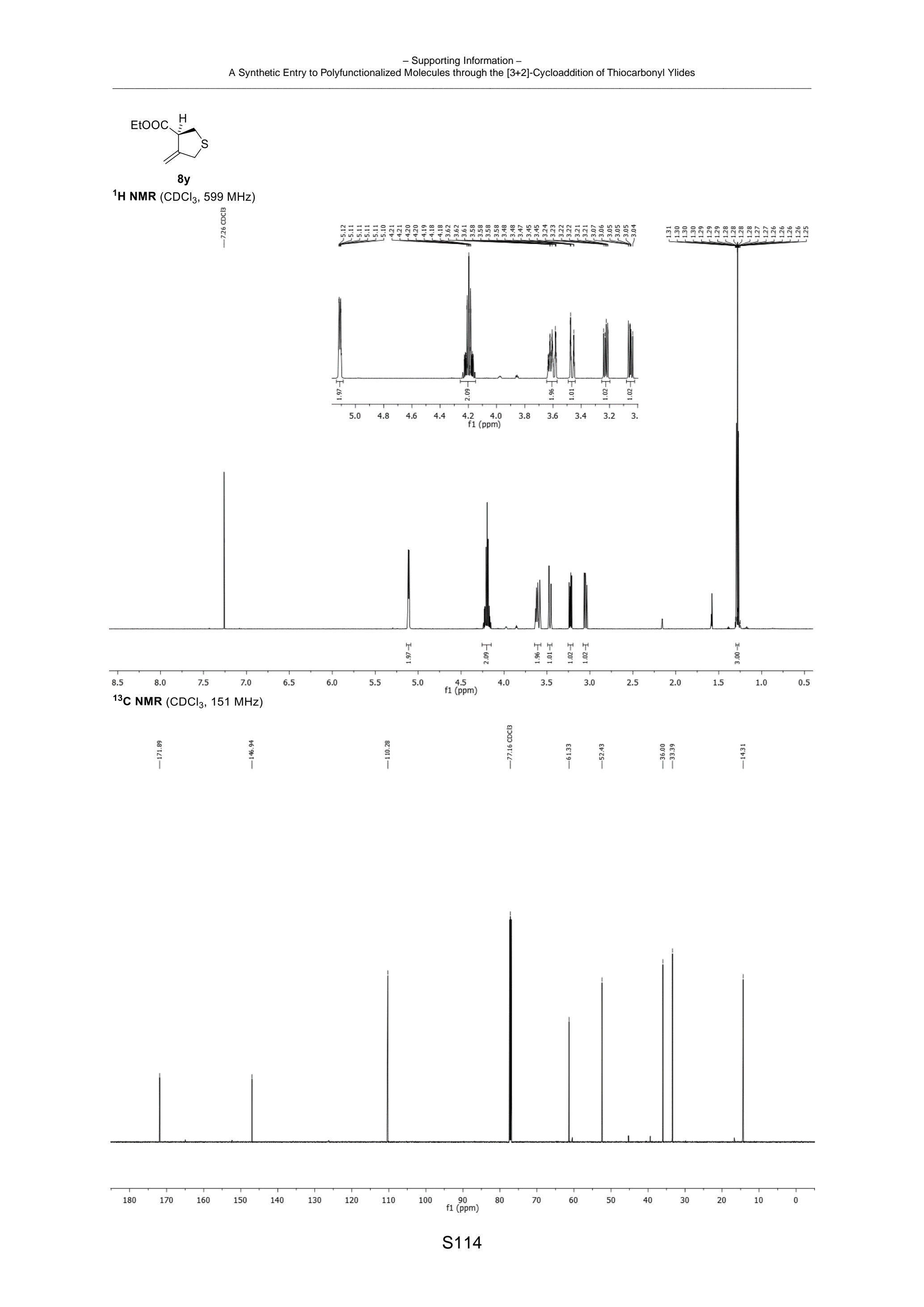




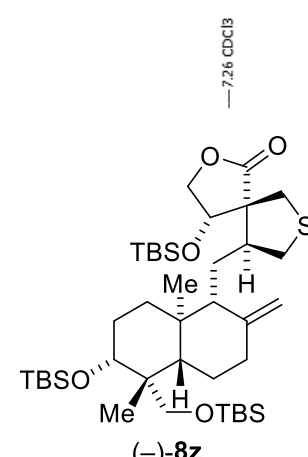

${ }^{1} \mathrm{H}$ NMR $\left(\mathrm{CDCl}_{3}, 599 \mathrm{MHz}\right)$

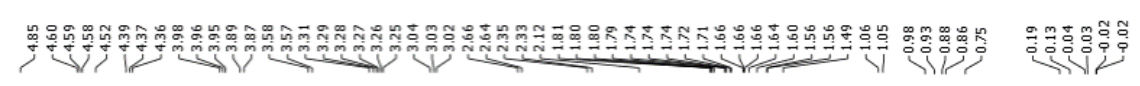
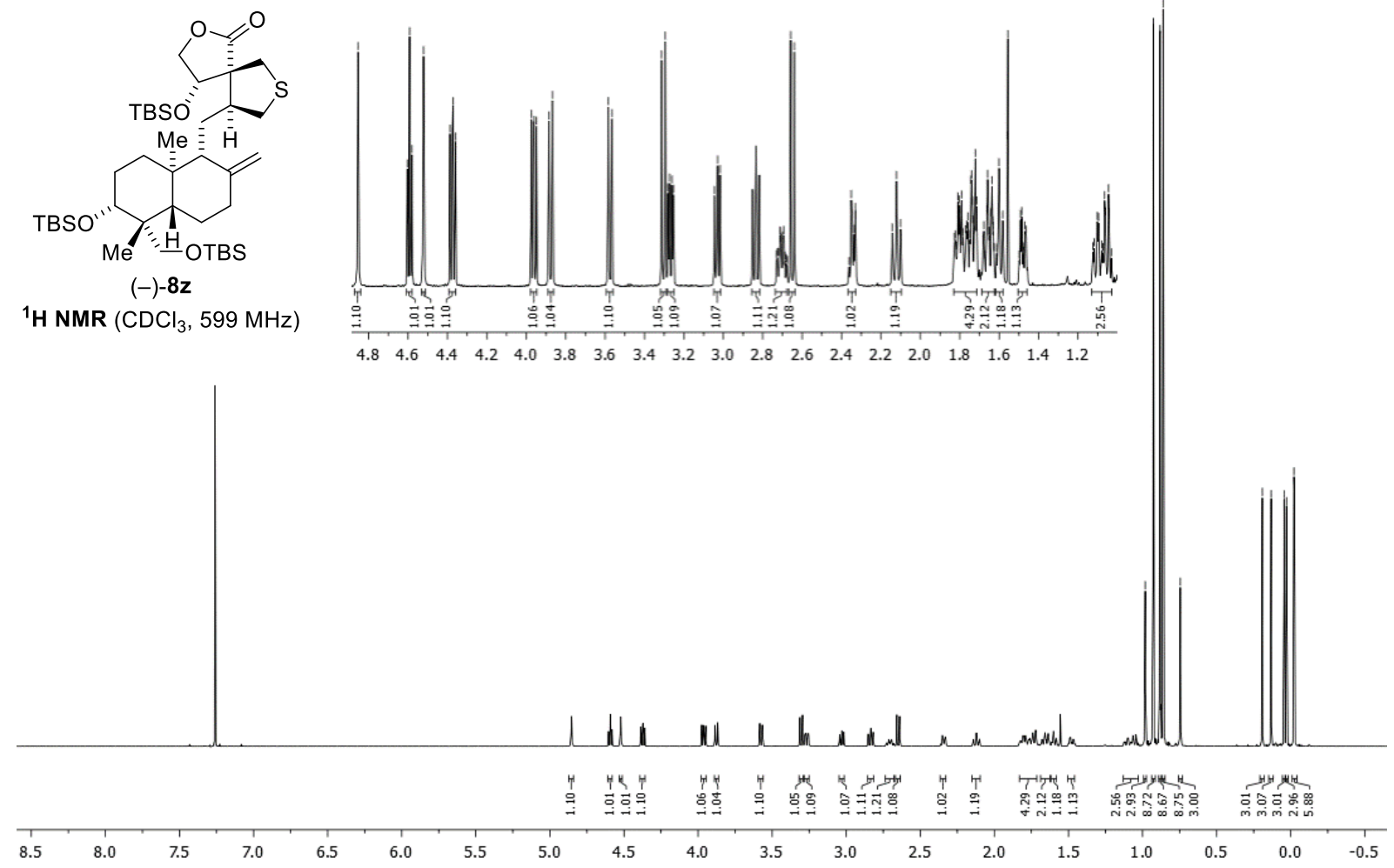

${ }^{13} \mathrm{C} \mathrm{NMR}\left(\mathrm{CDCl}_{3}, 151 \mathrm{MHz}\right)$

$\stackrel{\text { 总 }}{\stackrel{1}{i}}$

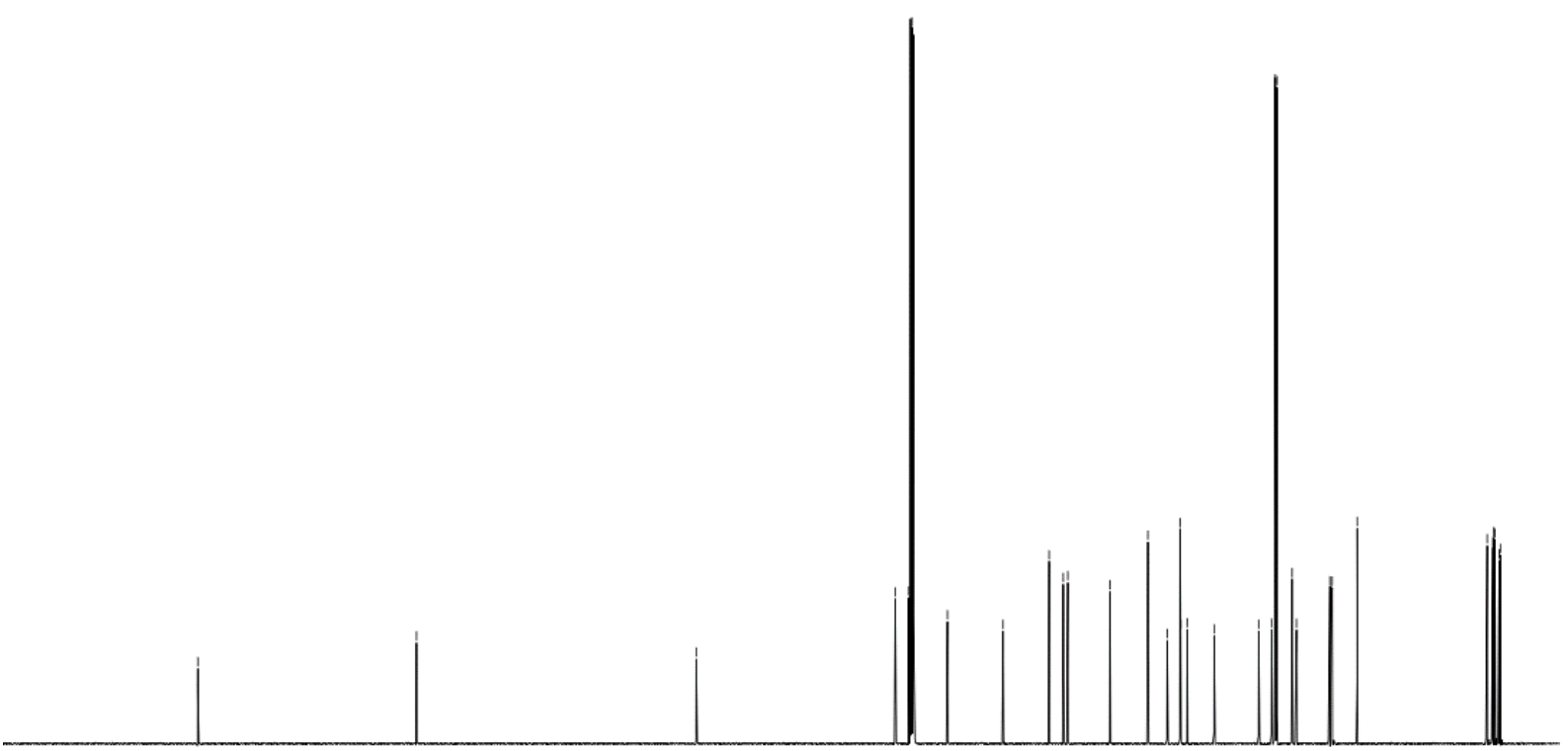




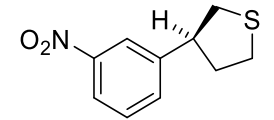

9a

${ }^{1} \mathrm{H}$ NMR $\left(\mathrm{CDCl}_{3}, 400 \mathrm{MHz}\right)$

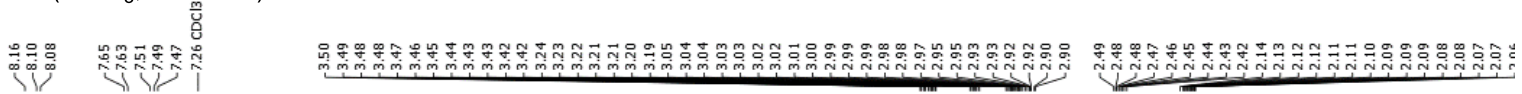

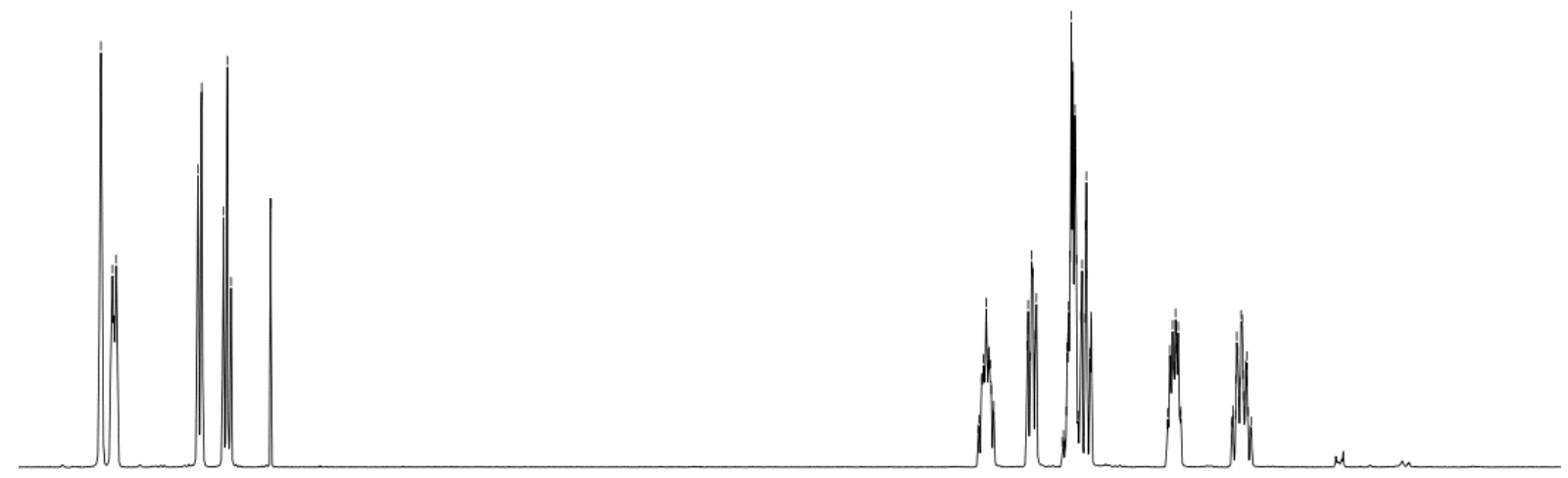

\begin{tabular}{|c|c|c|c|c|c|c|c|c|c|c|c|c|c|c|c|c|}
\hline & 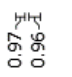 & 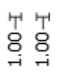 & & & & & & & & 'T & 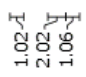 & $\begin{array}{l}\text { 'T } \\
\stackrel{2}{0}\end{array}$ & Ț & & & \\
\hline 8.5 & 8.0 & 7.5 & 7.0 & 6.5 & 6.0 & 5.5 & 5.0 & $\begin{array}{c}4.5 \\
\mathrm{f} 1(\mathrm{ppm})\end{array}$ & 4.0 & 3.5 & 3.0 & 2.5 & 2.0 & 1.5 & 1.0 & 0.5 \\
\hline
\end{tabular}

${ }^{13} \mathrm{C} \mathrm{NMR}\left(\mathrm{CDCl}_{3}, 101 \mathrm{MHz}\right)$
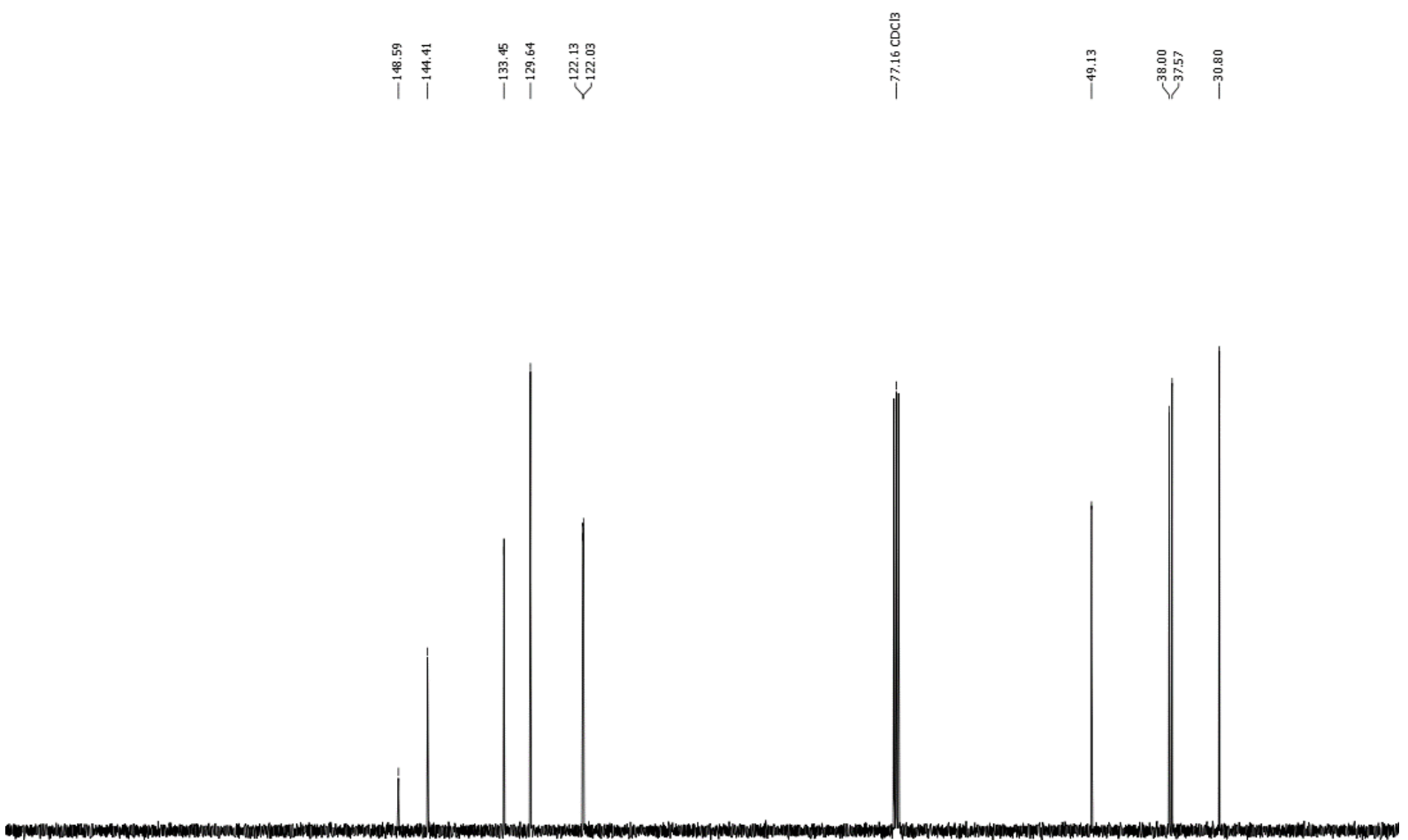

$\begin{array}{llllllllll}200 & 190 & 180 & 170 & 160 & 150 & 140 & 130 & 120 & \underset{\mathrm{f} 1(\mathrm{ppm})}{110}\end{array}$ 
<smiles>Fc1ccc([C@H]2CCSC2)cc1</smiles>

9b

${ }^{1} \mathrm{H}$ NMR $\left(\mathrm{CDCl}_{3}, 400 \mathrm{MHz}\right)$

范

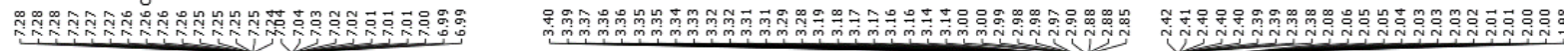

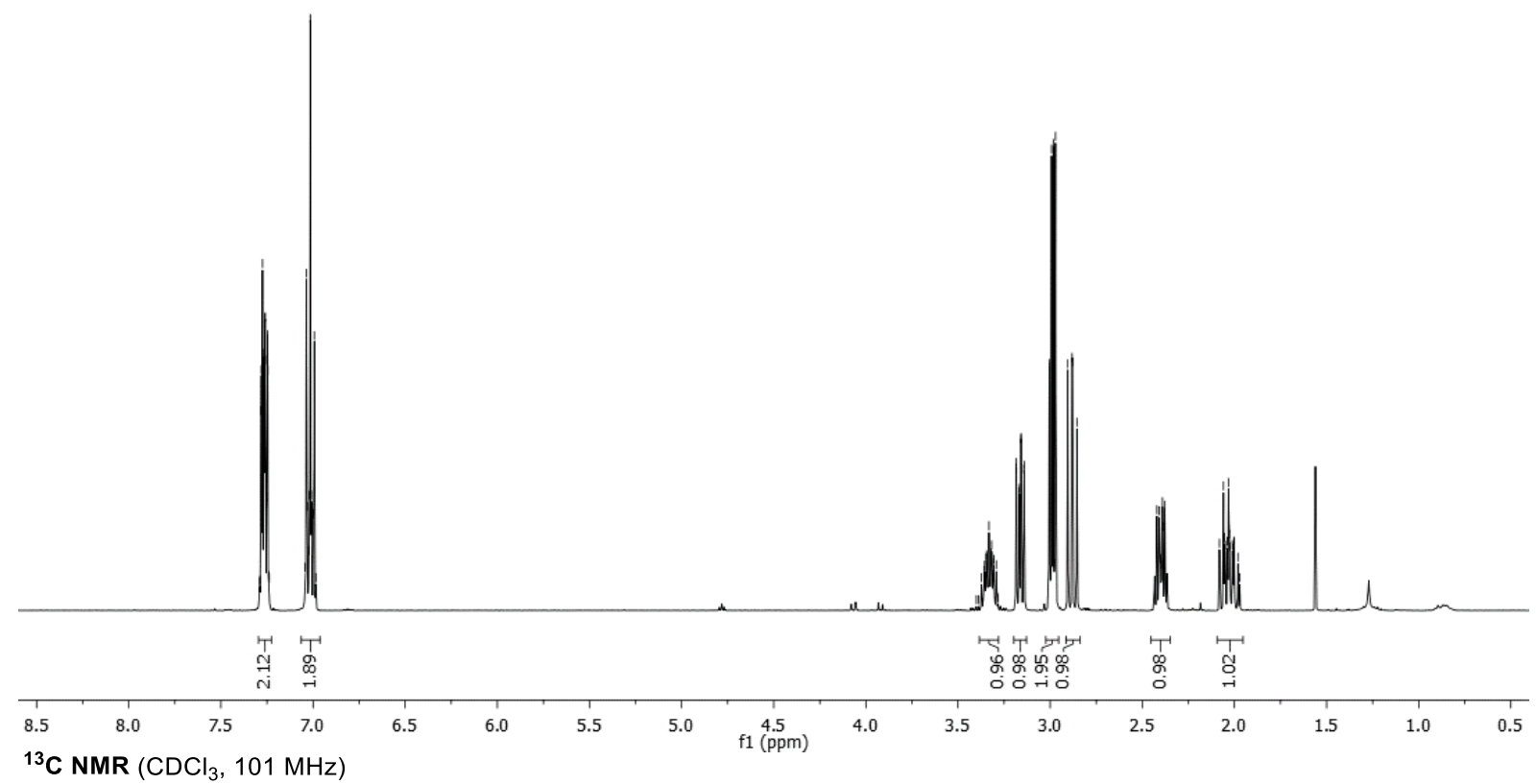


${ }^{19} \mathrm{~F} \mathrm{NMR}\left(\mathrm{CDCl}_{3}, 376 \mathrm{MHz}\right)$ 


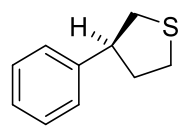

9c

${ }^{1} \mathrm{H} \mathrm{NMR}\left(\mathrm{CDCl}_{3}, 400 \mathrm{MHz}\right)$

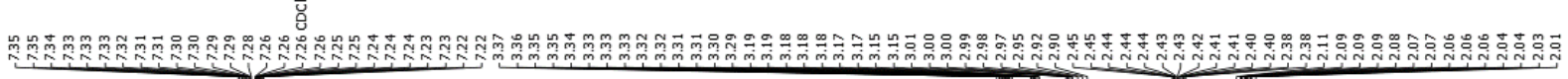

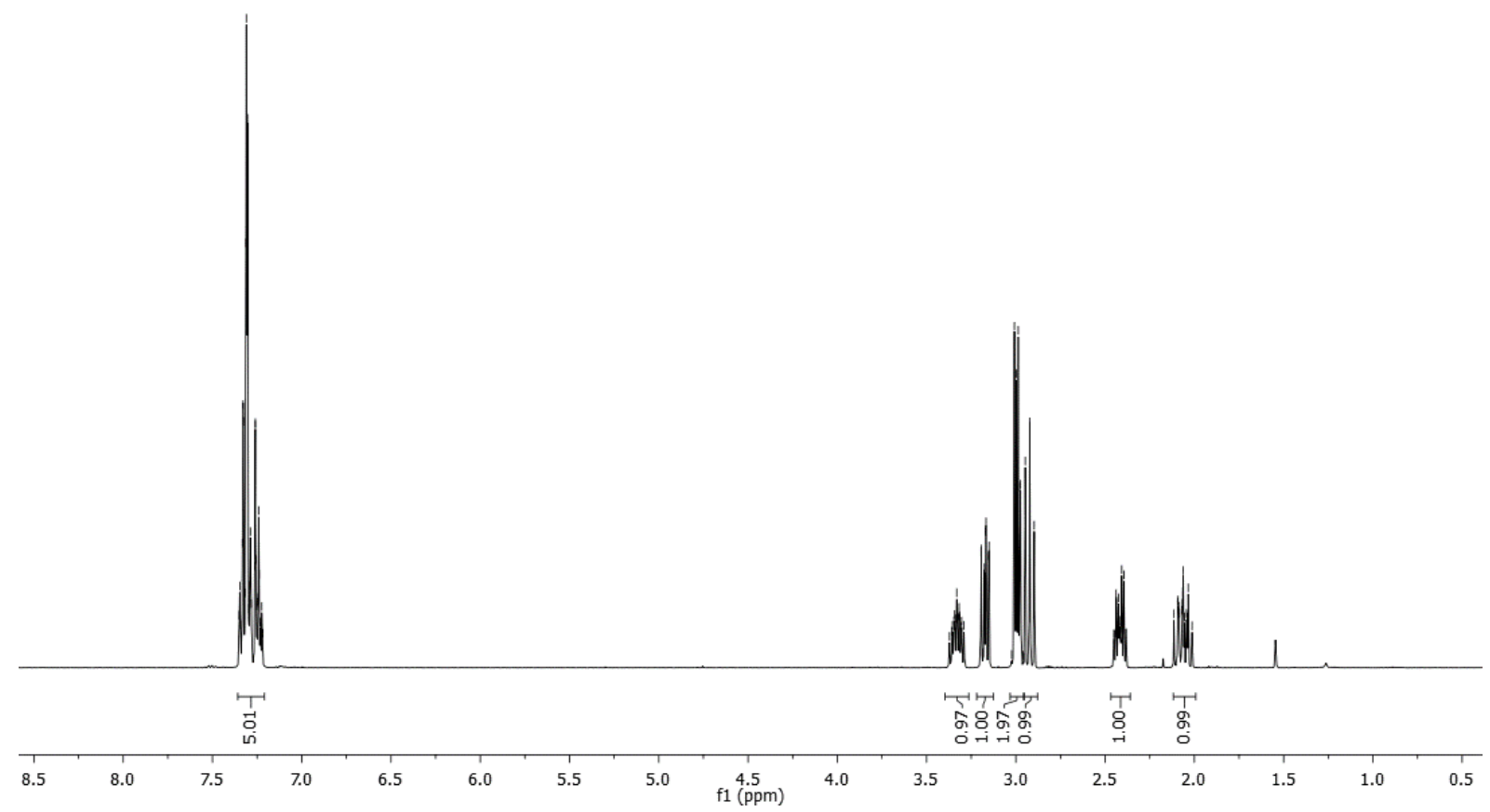

${ }^{13} \mathrm{C}$ NMR $\left(\mathrm{CDCl}_{3}, 101 \mathrm{MHz}\right)$

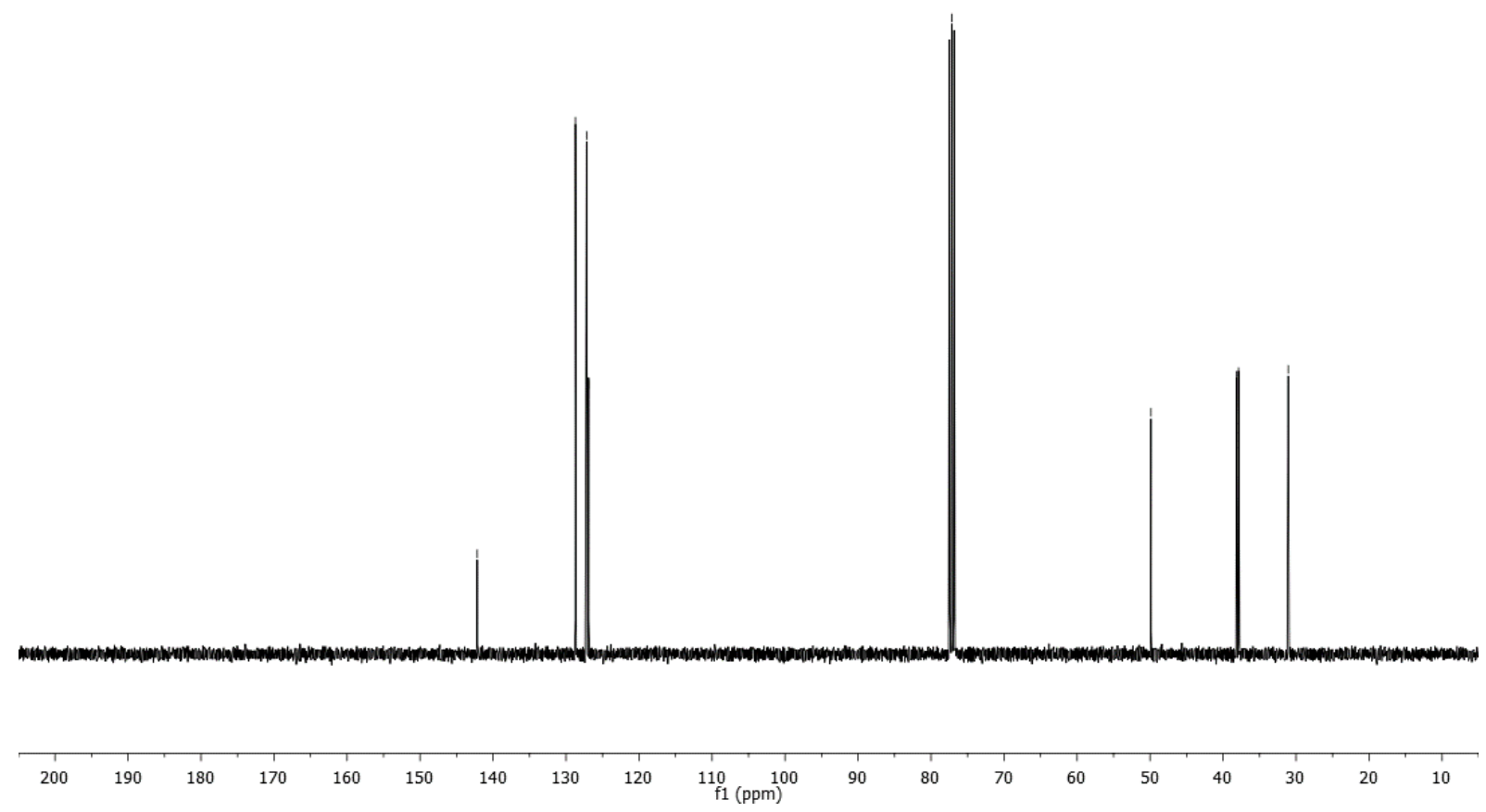




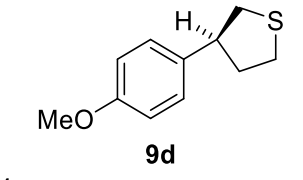

${ }^{1} \mathrm{H}$ NMR $\left(\mathrm{CDCl}_{3}, 400 \mathrm{MHz}\right)$

䒿

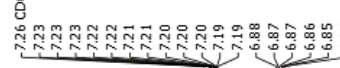

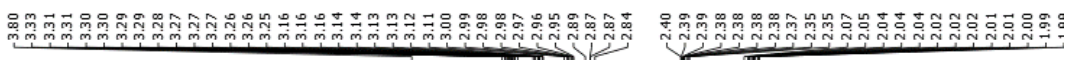

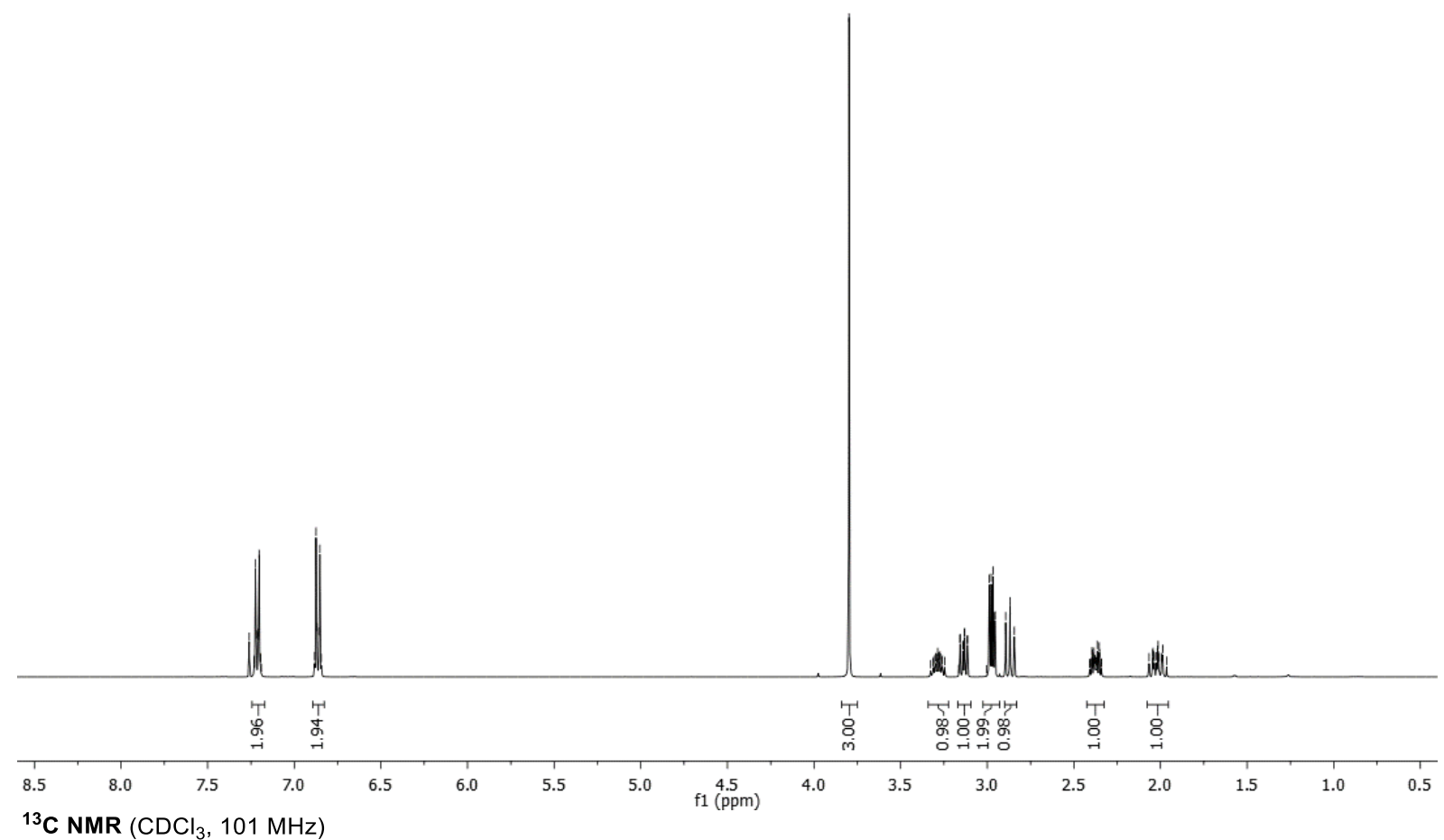

${ }^{13} \mathrm{C}$ NMR $\left(\mathrm{CDCl}_{3}, 101 \mathrm{MHz}\right)$

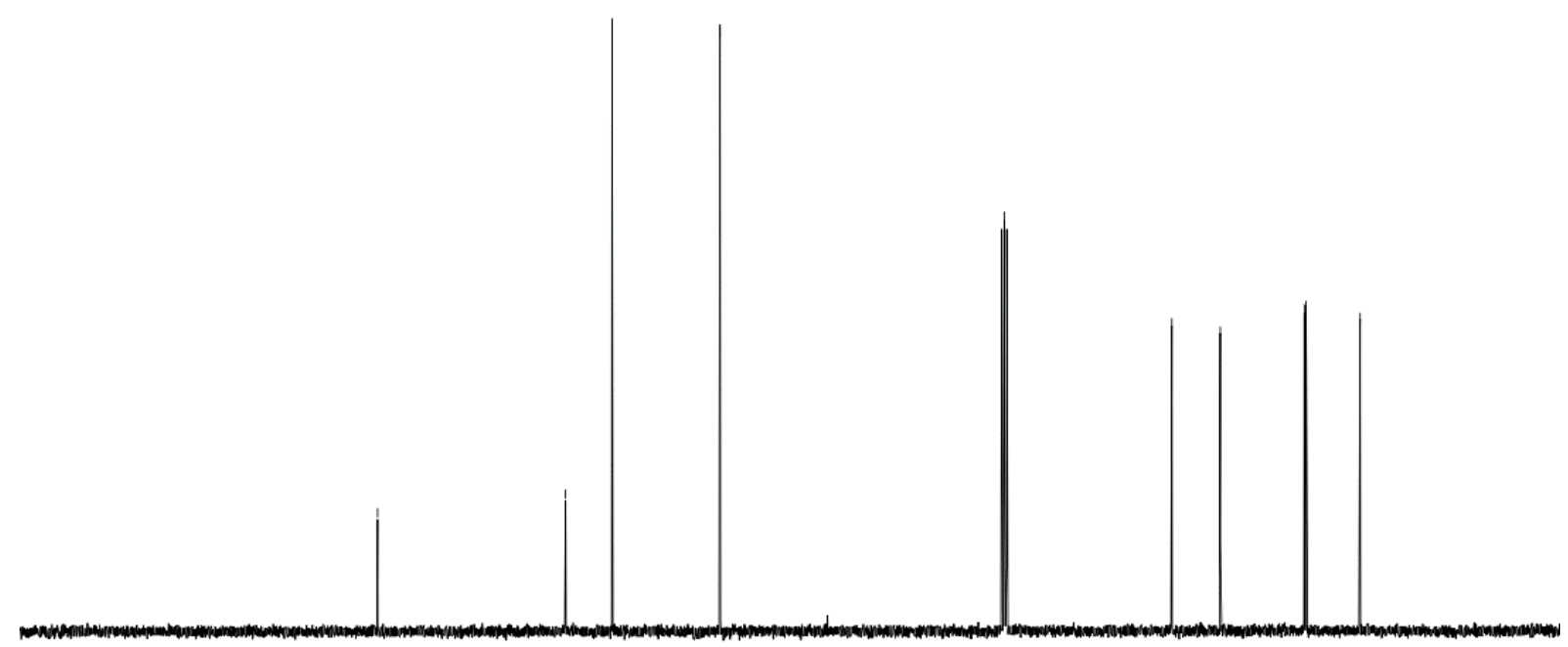




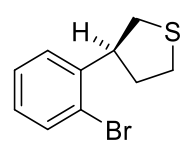

$9 e$

${ }^{1} \mathrm{H}$ NMR $\left(\mathrm{CDCl}_{3}, 400 \mathrm{MHz}\right)$

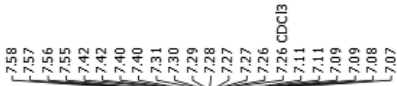

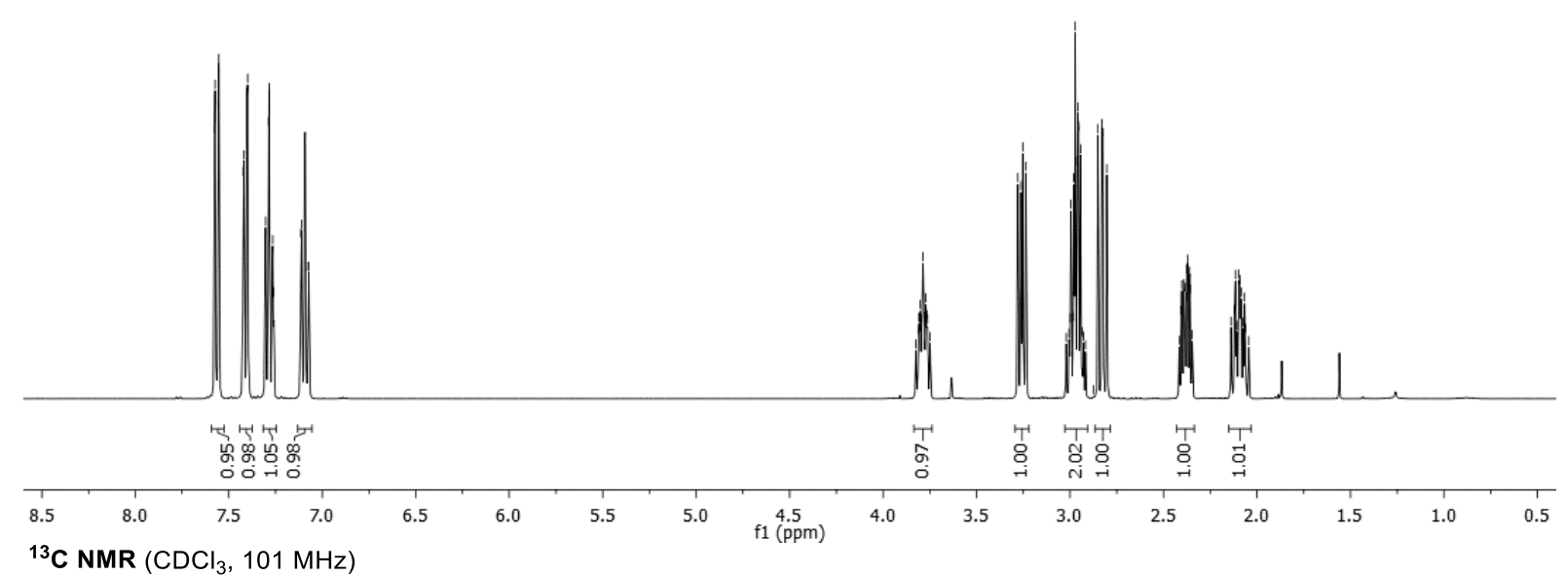

${ }^{13} \mathrm{C}$ NMR $\left(\mathrm{CDCl}_{3}, 101 \mathrm{MHz}\right)$
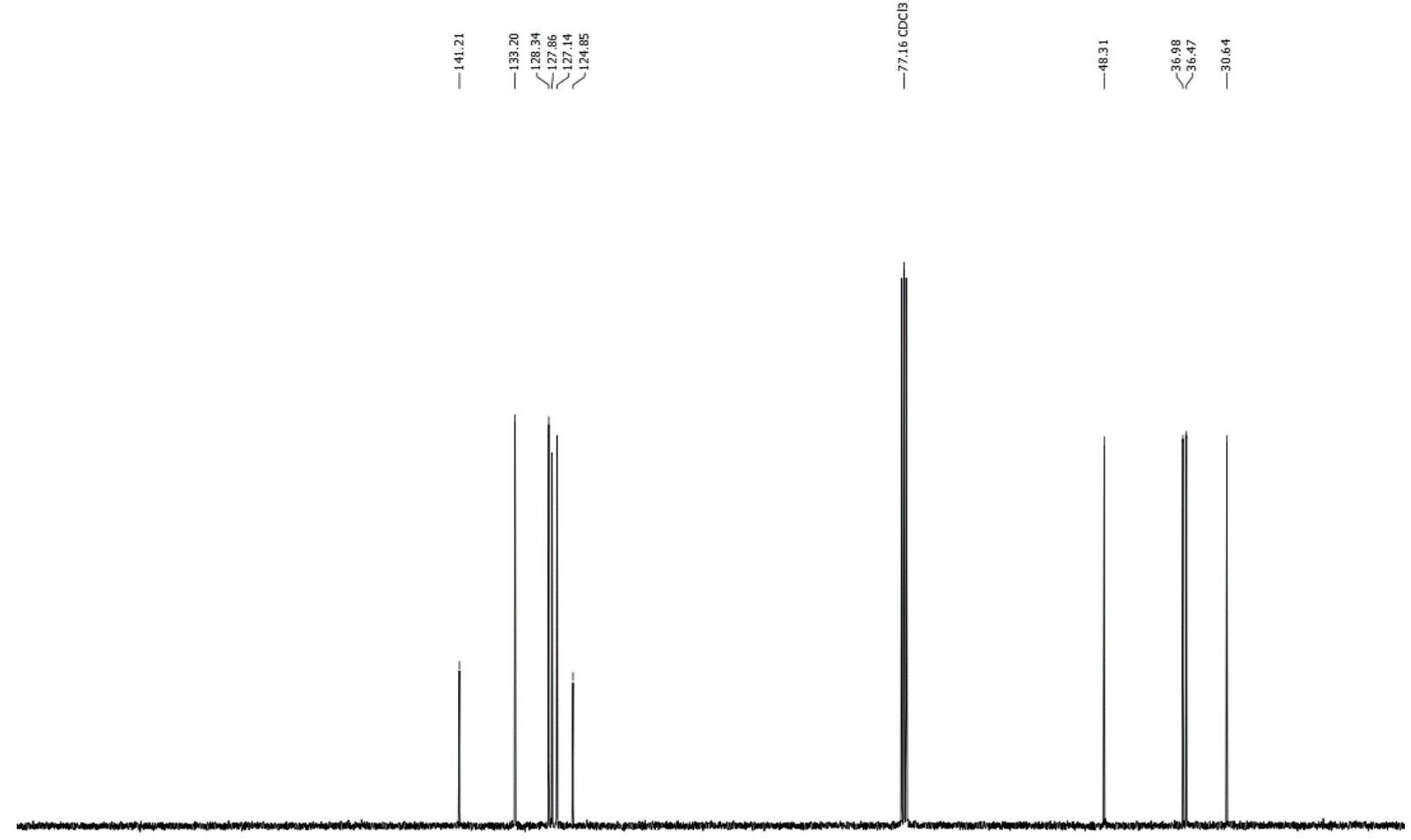

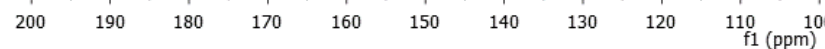




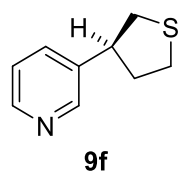

${ }^{1} \mathrm{H} \mathrm{NMR}\left(\mathrm{CDCl}_{3}, 400 \mathrm{MHz}\right)$

学

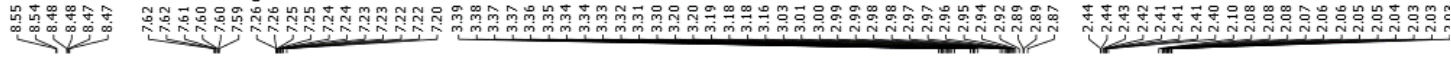

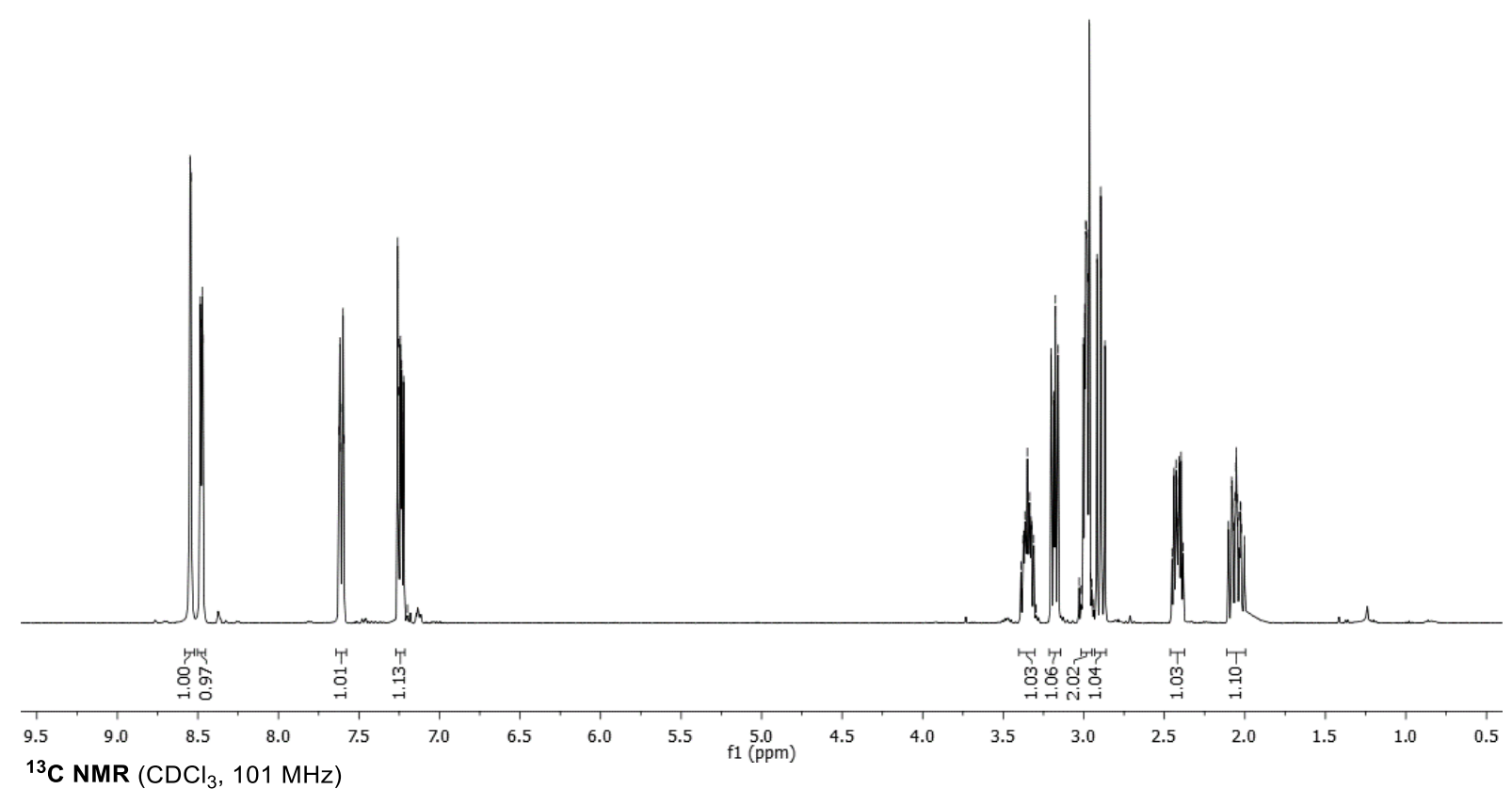

${ }^{13} \mathrm{C}$ NMR $\left(\mathrm{CDCl}_{3}, 101 \mathrm{MHz}\right)$

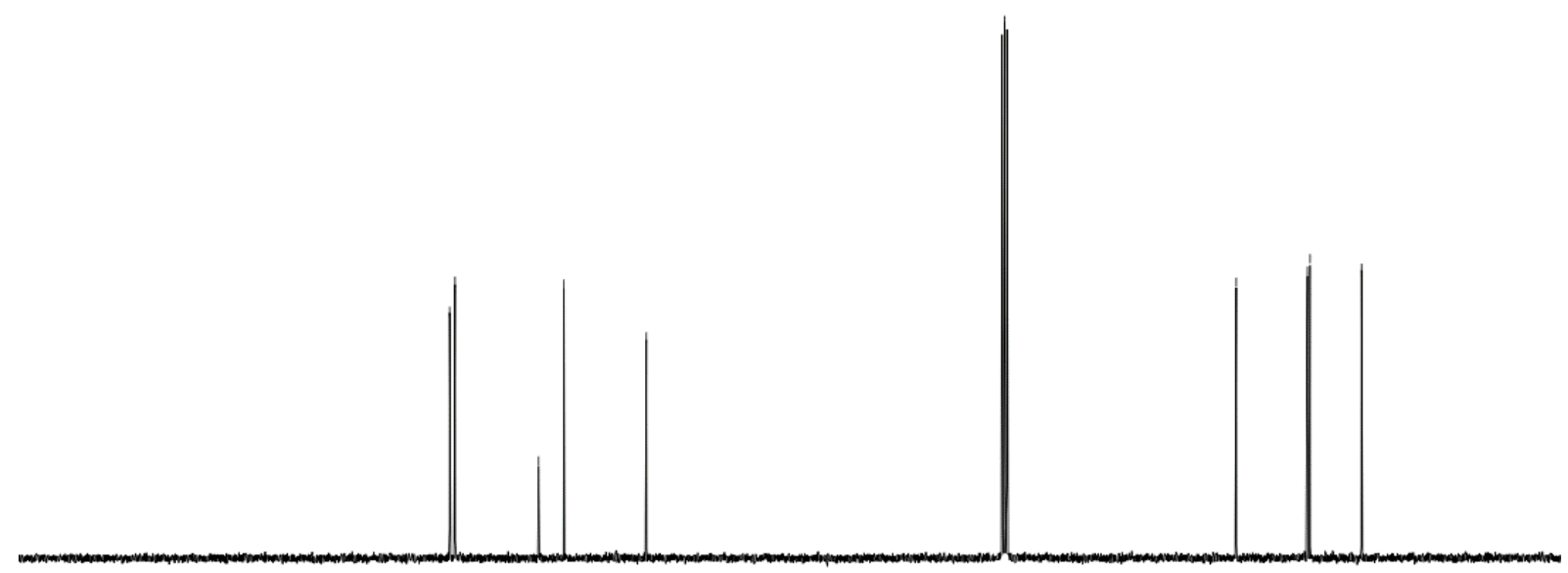

$200 \quad 190$ 


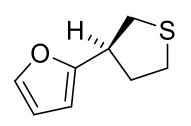

9g

${ }^{1} \mathrm{H} \mathrm{NMR}\left(\mathrm{CDCl}_{3}, 400 \mathrm{MHz}\right)$
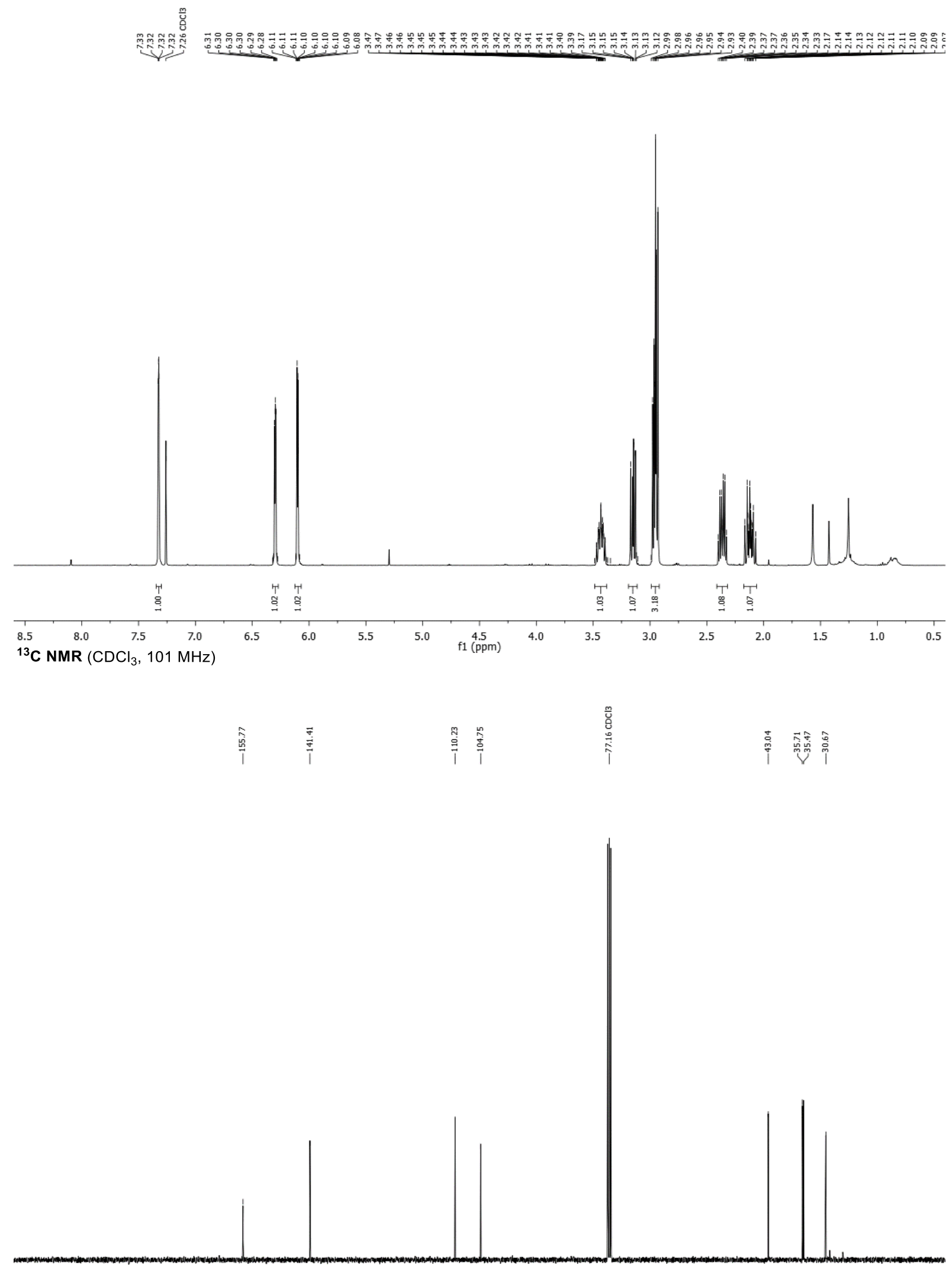

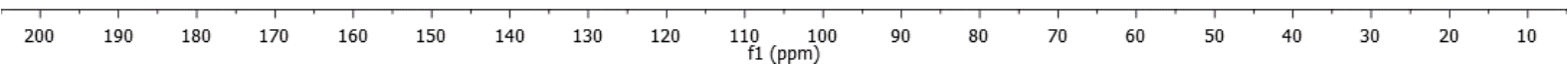




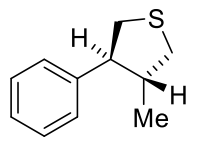

$9 \mathbf{h}$

${ }^{1} \mathrm{H}$ NMR $\left(\mathrm{CDCl}_{3}, 400 \mathrm{MHz}\right)$

觉

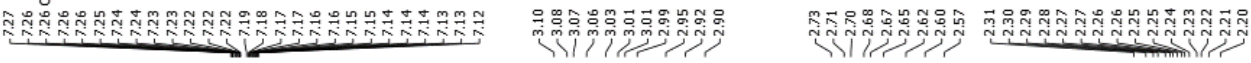
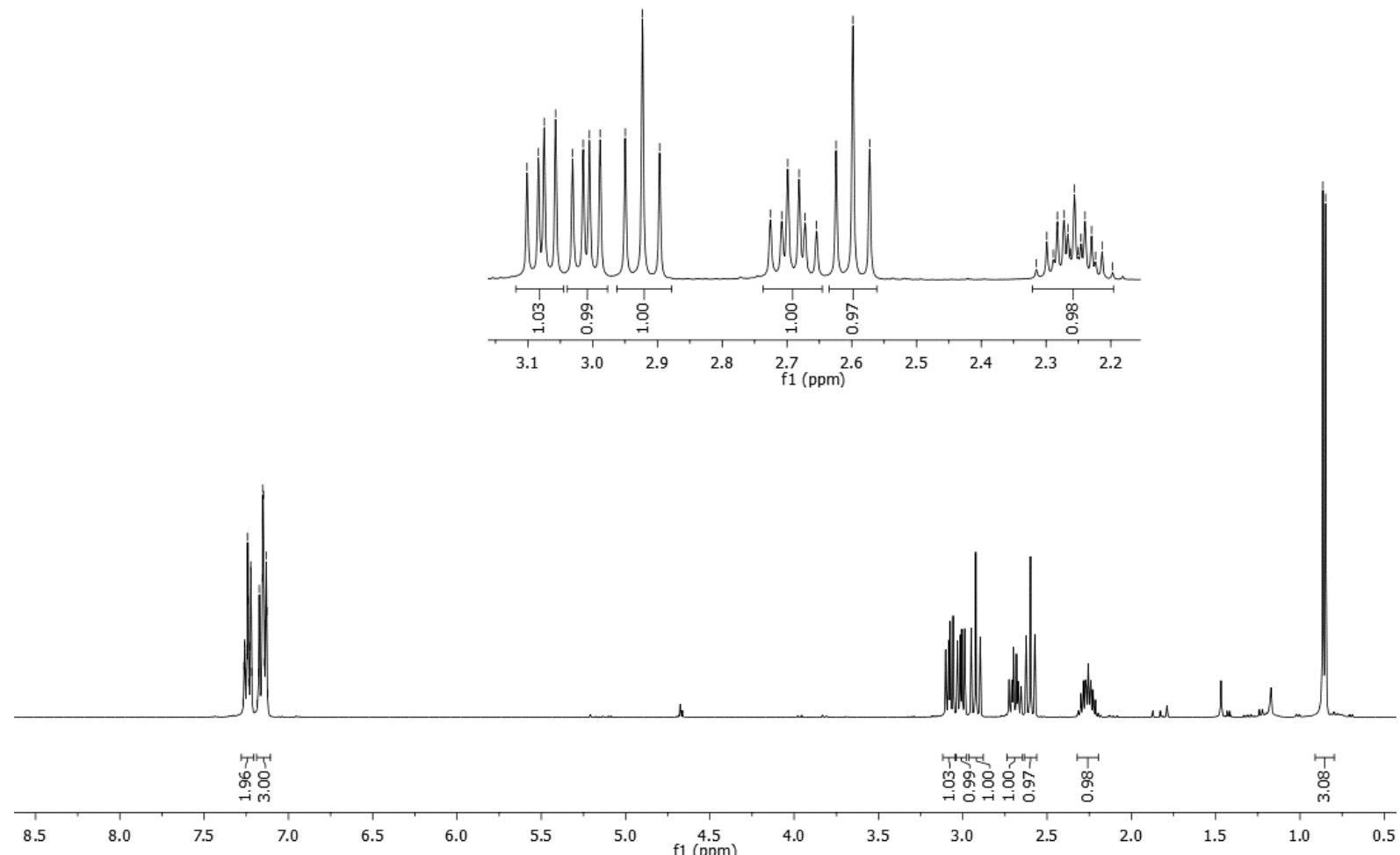

${ }^{13} \mathrm{C}$ NMR $\left(\mathrm{CDCl}_{3}, 101 \mathrm{MHz}\right)$

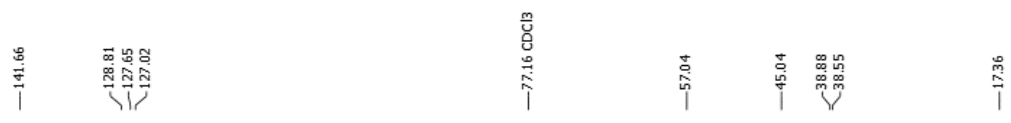

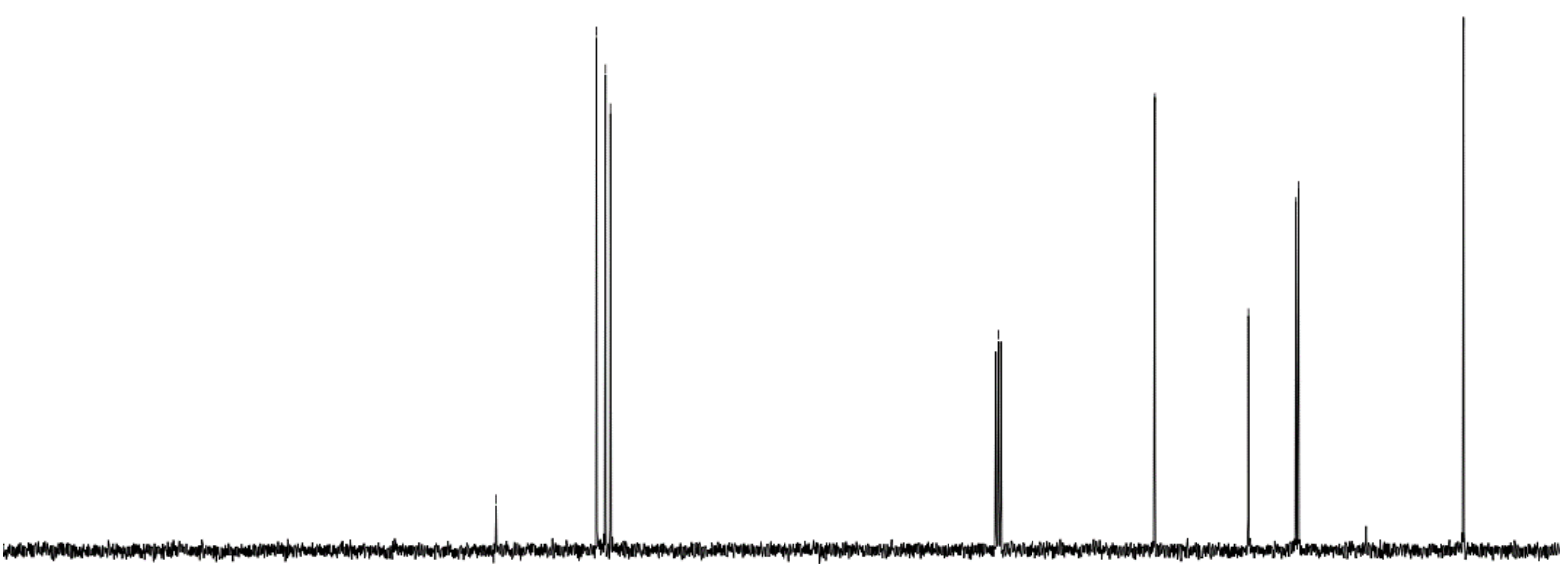

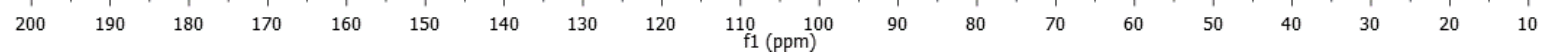




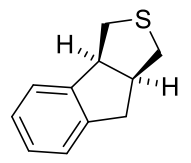

$9 \mathbf{i}$

${ }^{1} \mathrm{H}$ NMR $\left(\mathrm{CDCl}_{3}, 400 \mathrm{MHz}\right)$

$\frac{\mathrm{m}}{\mathrm{u}}$

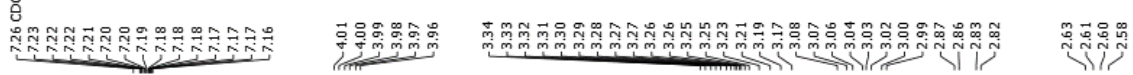
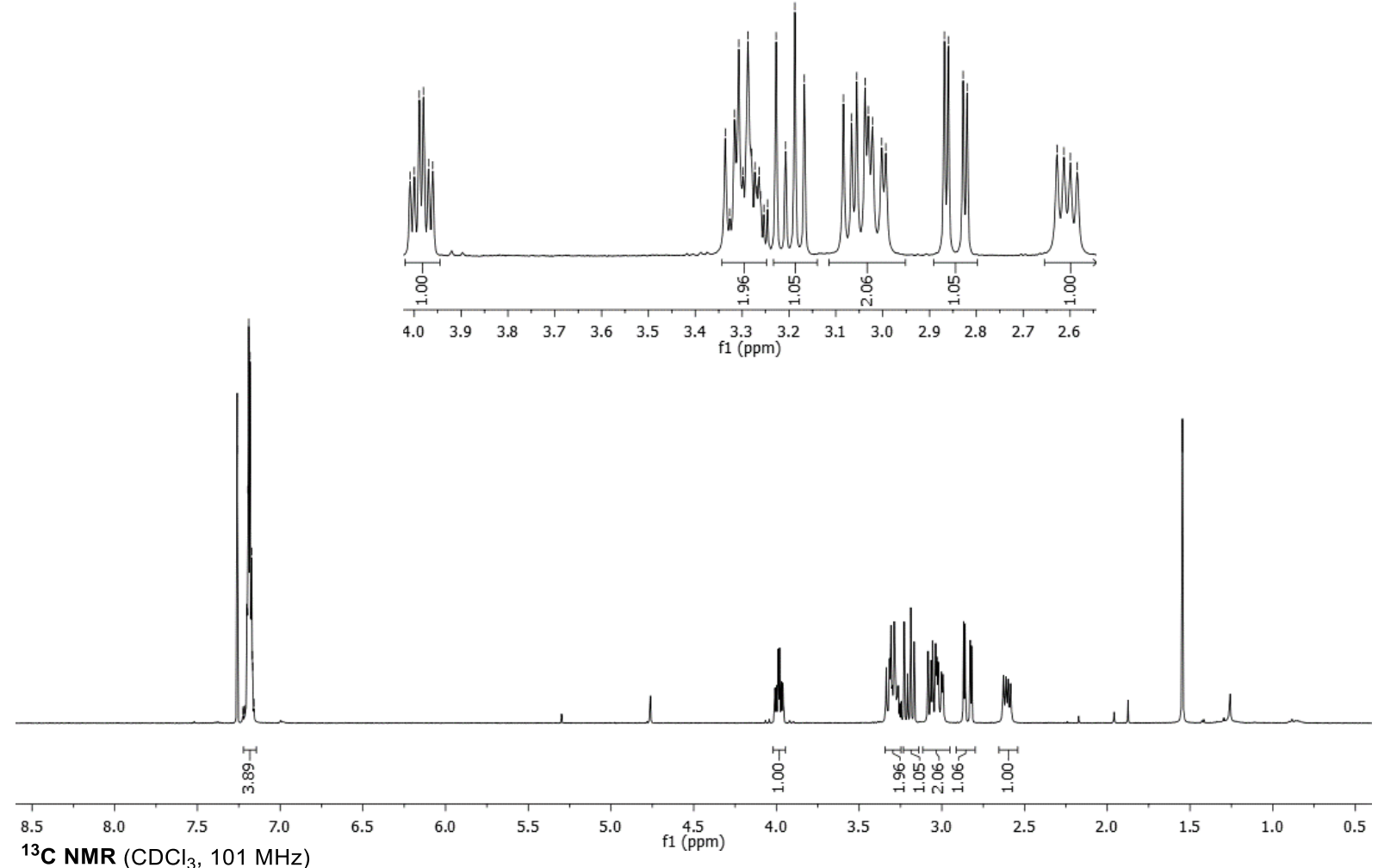

${ }^{13} \mathrm{C}$ NMR $\left(\mathrm{CDCl}_{3}, 101 \mathrm{MHz}\right)$

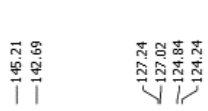

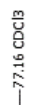

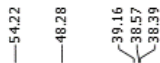

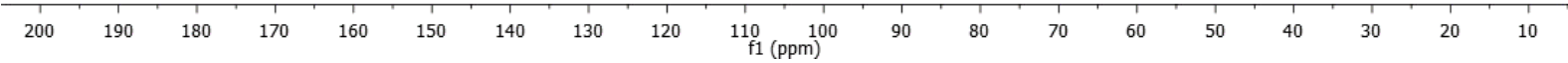




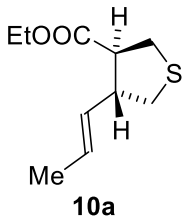

${ }^{1} \mathrm{H}$ NMR $\left(\mathrm{CDCl}_{3}, 400 \mathrm{MHz}\right)$
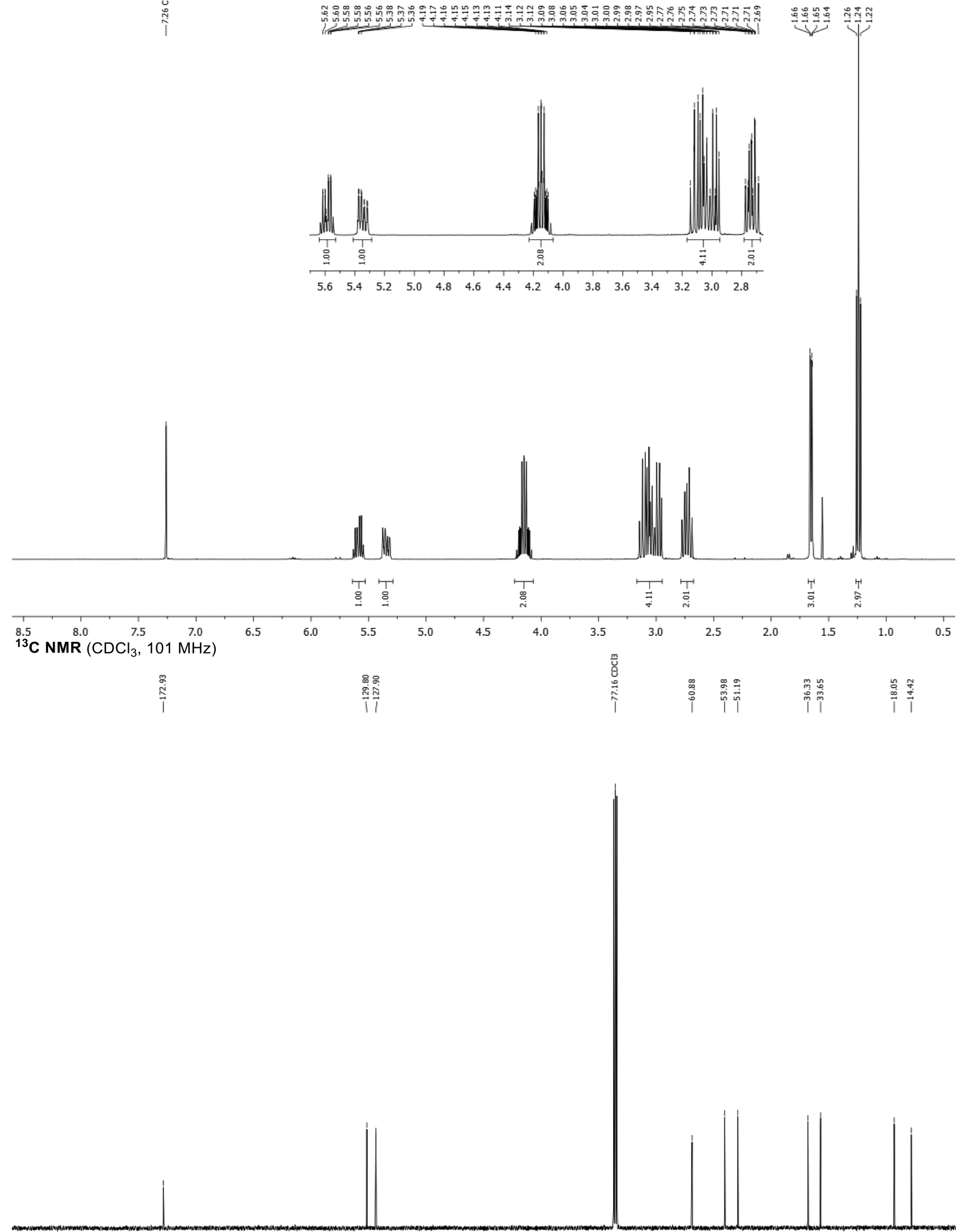

200
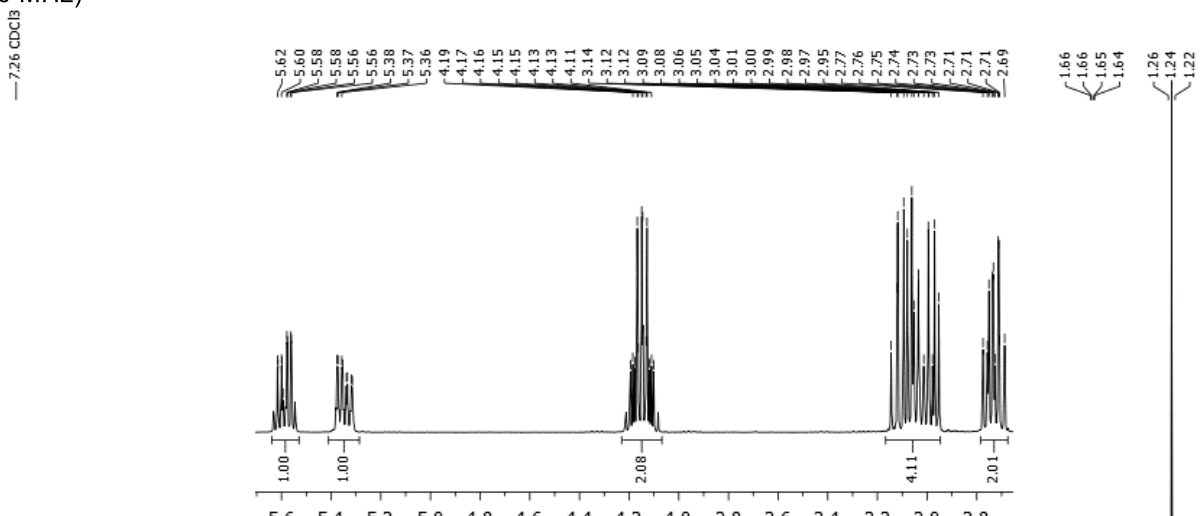


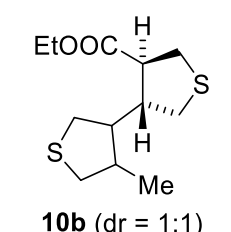

${ }^{1} \mathbf{H}$ NMR $\left(\mathrm{CDCl}_{3}, 400 \mathrm{MHz}\right)$

远

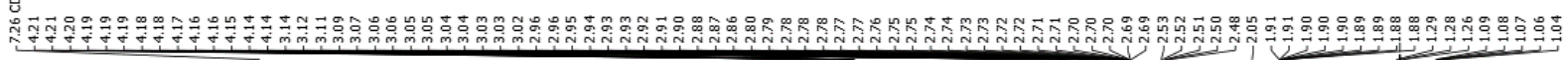
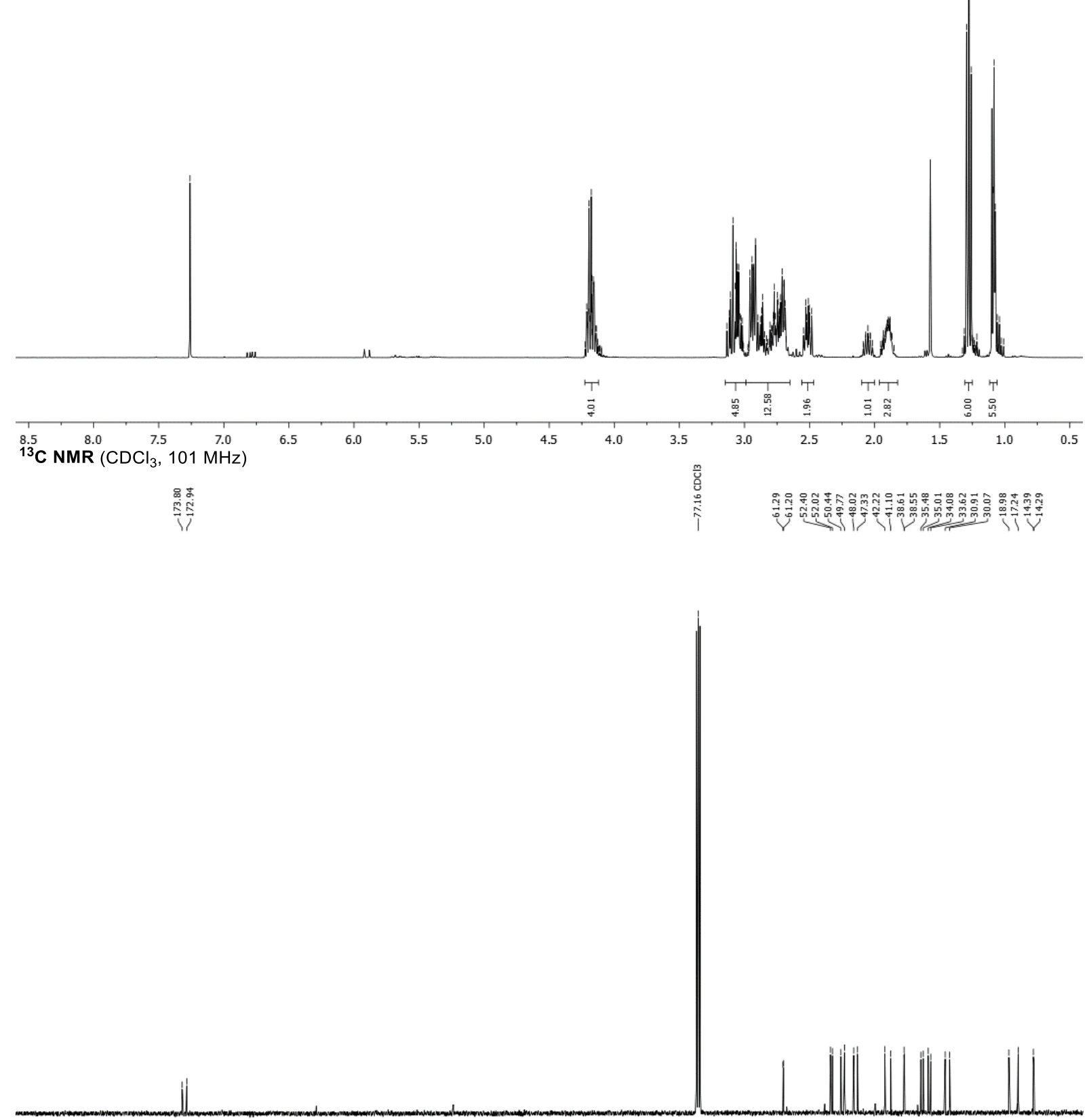


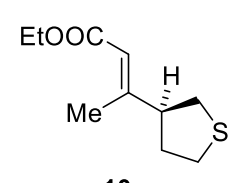

$10 \mathrm{c}$

${ }^{1} \mathrm{H}$ NMR $\left(\mathrm{CDCl}_{3}, 400 \mathrm{MHz}\right)$
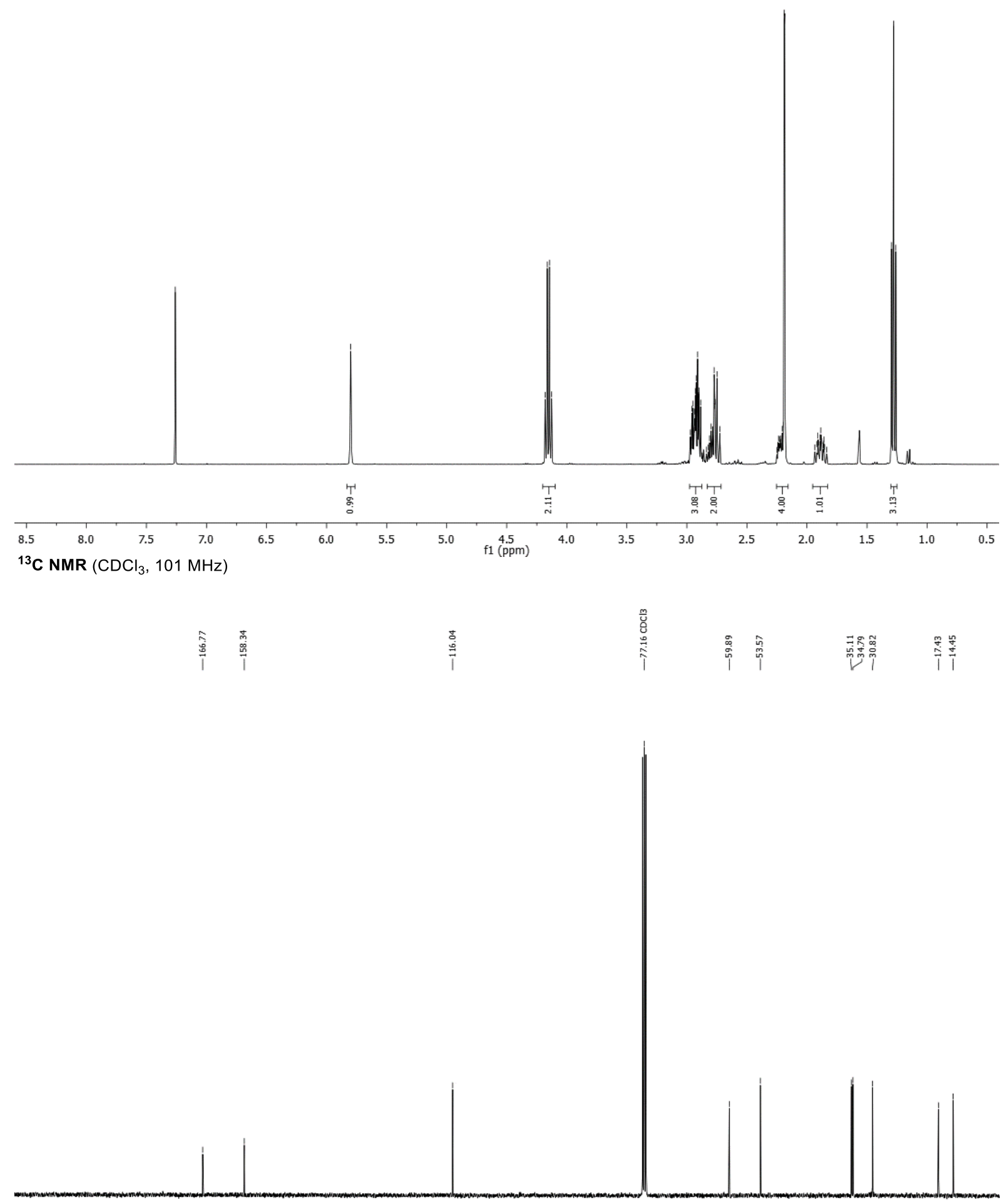

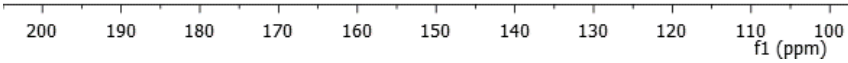




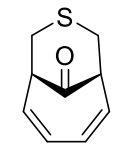

11

${ }^{1} \mathrm{H}$ NMR $\left(\mathrm{CDCl}_{3}, 400 \mathrm{MHz}\right)$

$\underbrace{7}$

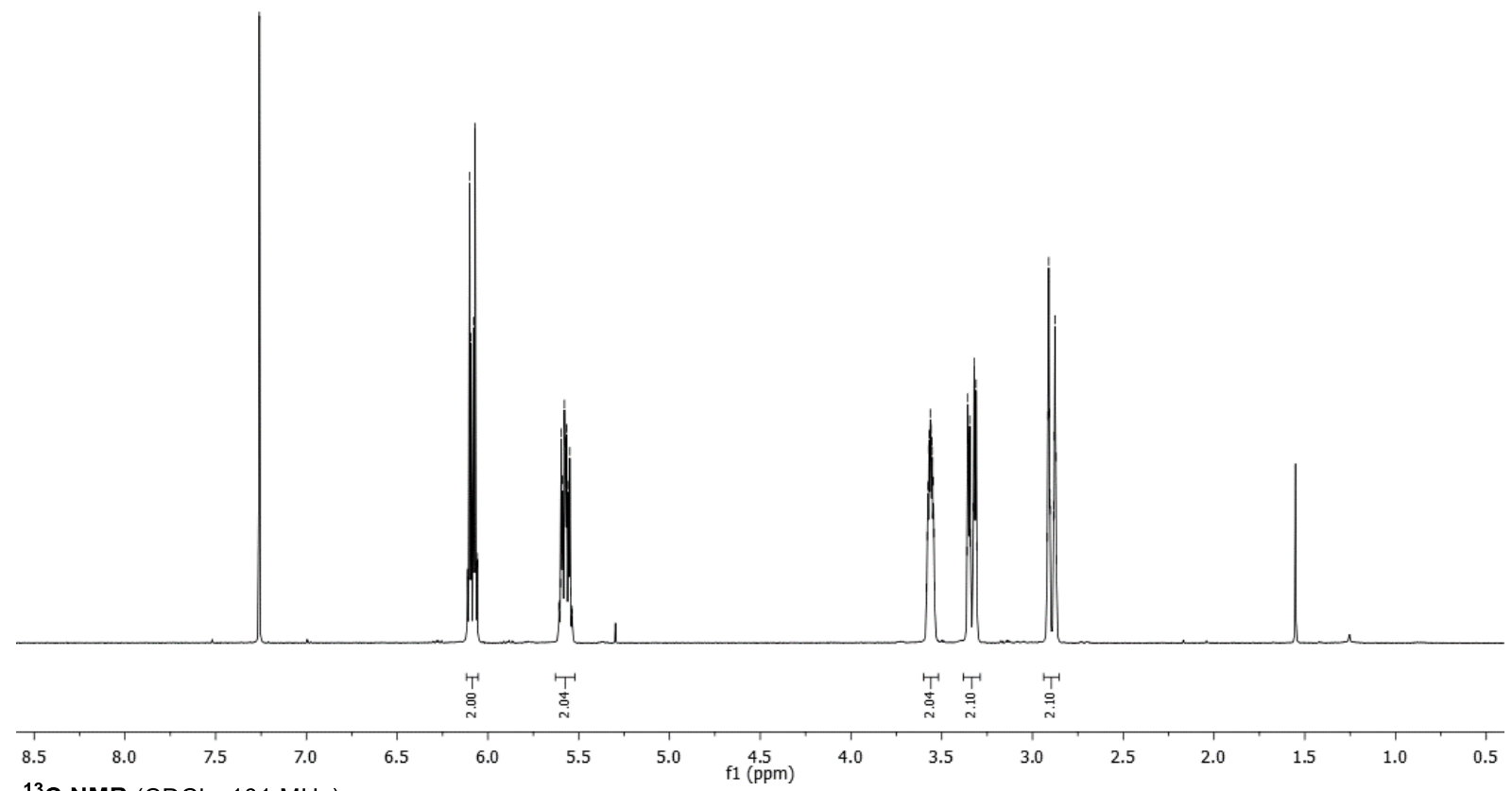

${ }^{13} \mathrm{C}$ NMR $\left(\mathrm{CDCl}_{3}, 101 \mathrm{MHz}\right)$

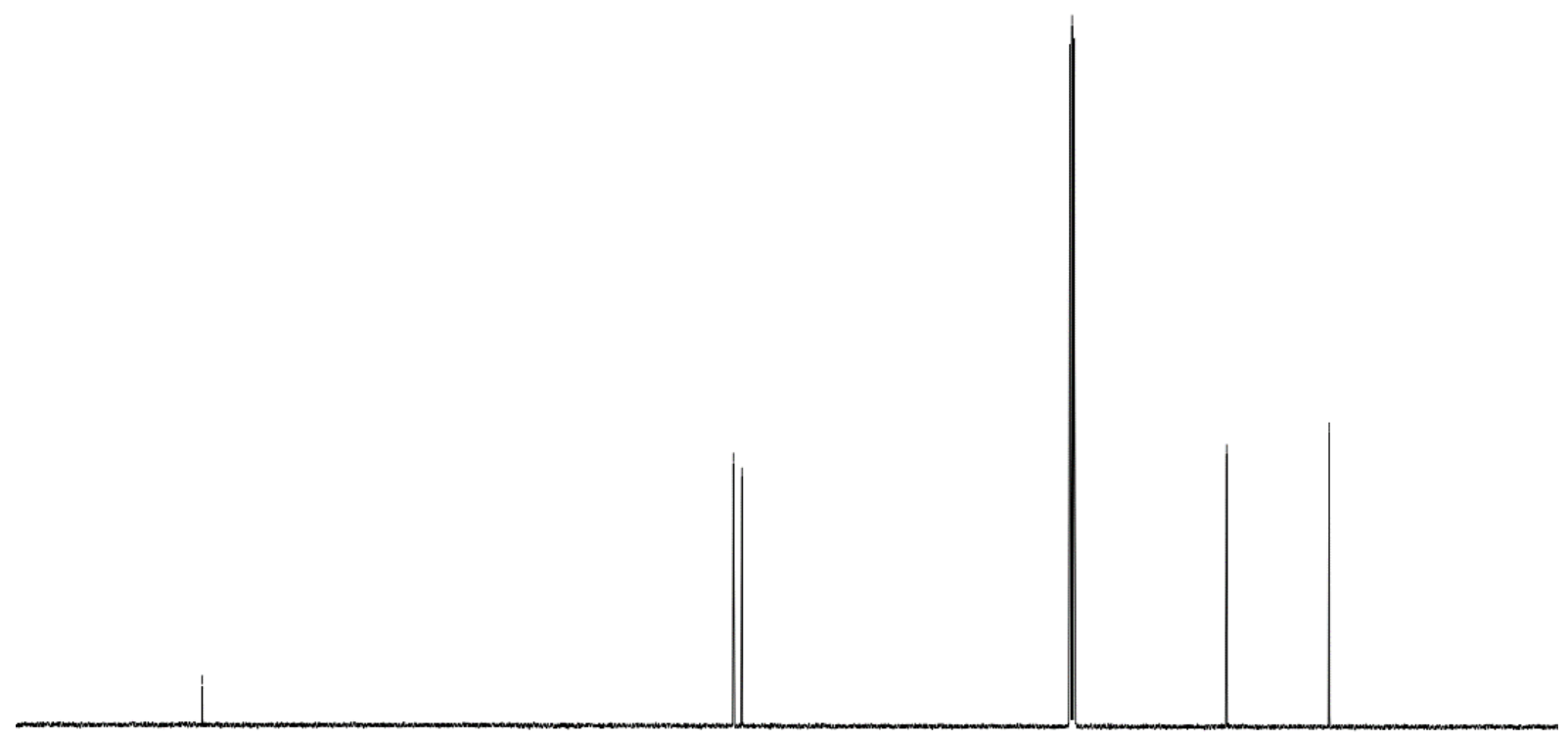

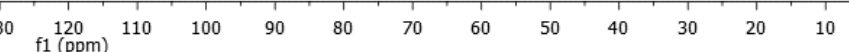


<smiles>CC(=O)C1=C(c2ccccc2)CSC1</smiles>

$12 \mathrm{a}$

${ }^{1} \mathrm{H}$ NMR $\left(\mathrm{CDCl}_{3}, 400 \mathrm{MHz}\right)$

$\frac{\mathrm{m}}{\mathrm{U}}$

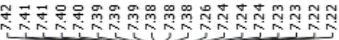

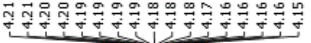

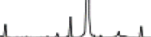

'⿳宀'

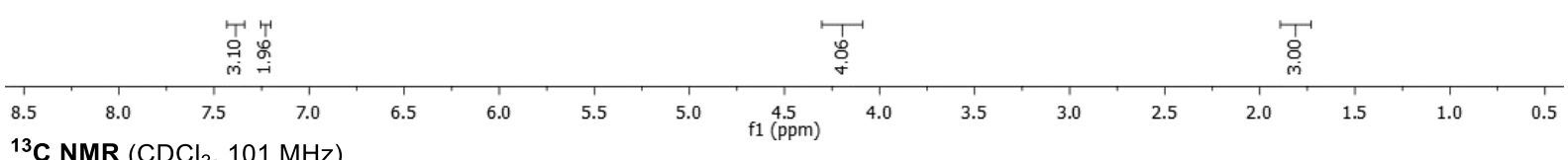

${ }^{13} \mathrm{C}$ NMR $\left(\mathrm{CDCl}_{3}, 101 \mathrm{MHz}\right)$

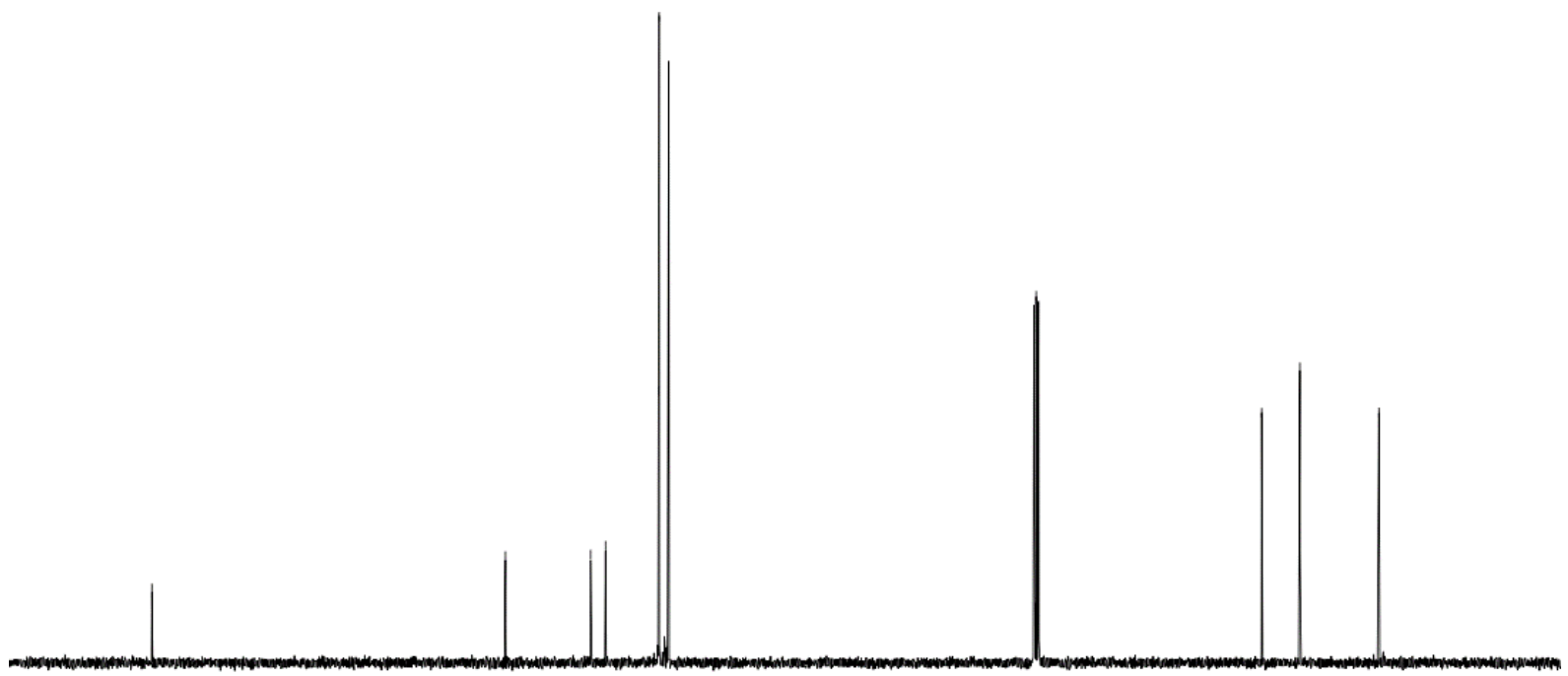

$\begin{array}{llllllllll}210 & 200 & 190 & 180 & 170 & 160 & 150 & 140 & 130 & 120 \\ \mathrm{f} 1(\mathrm{ppm})\end{array}$ 
<smiles>N#CC1=C(c2ccccc2)CSC1</smiles>

$12 b$

${ }^{1} \mathrm{H}$ NMR $\left(\mathrm{CDCl}_{3}, 400 \mathrm{MHz}\right)$

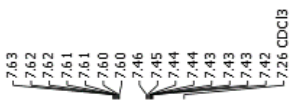

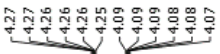

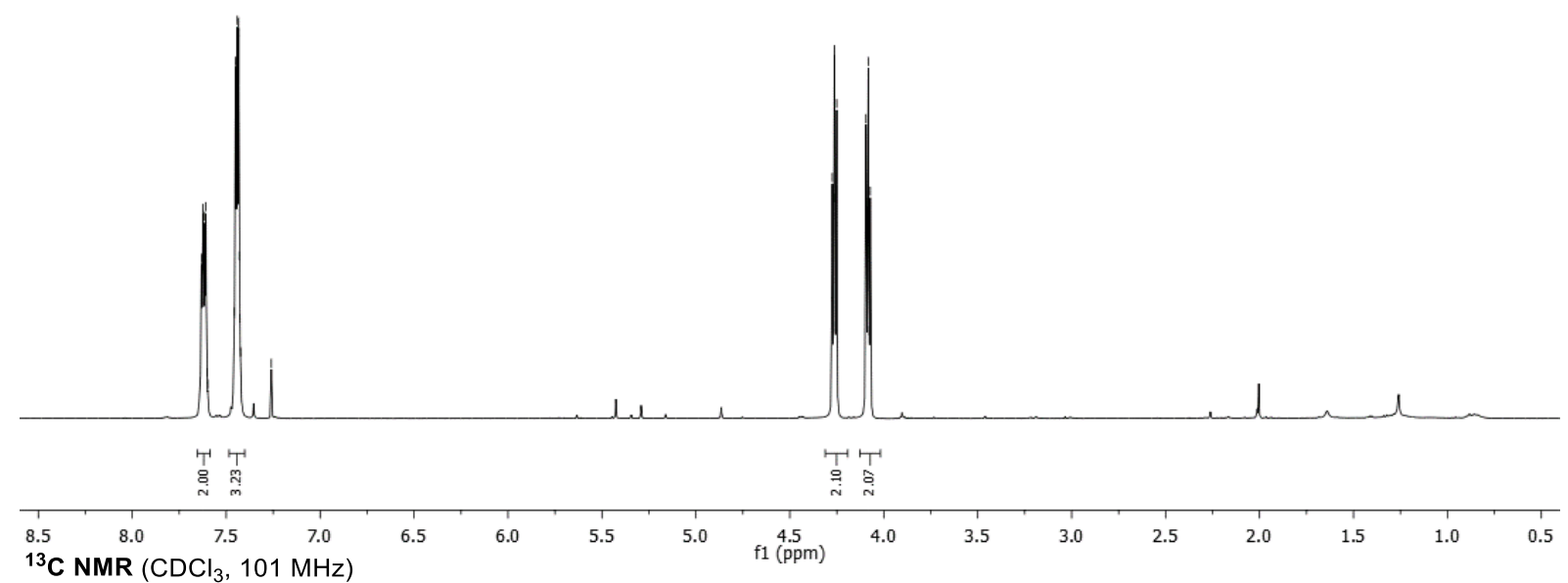

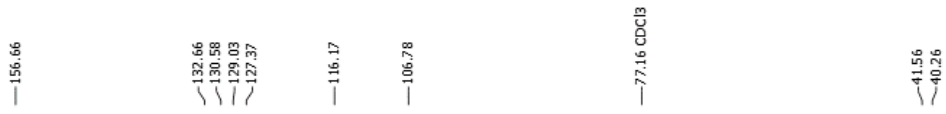

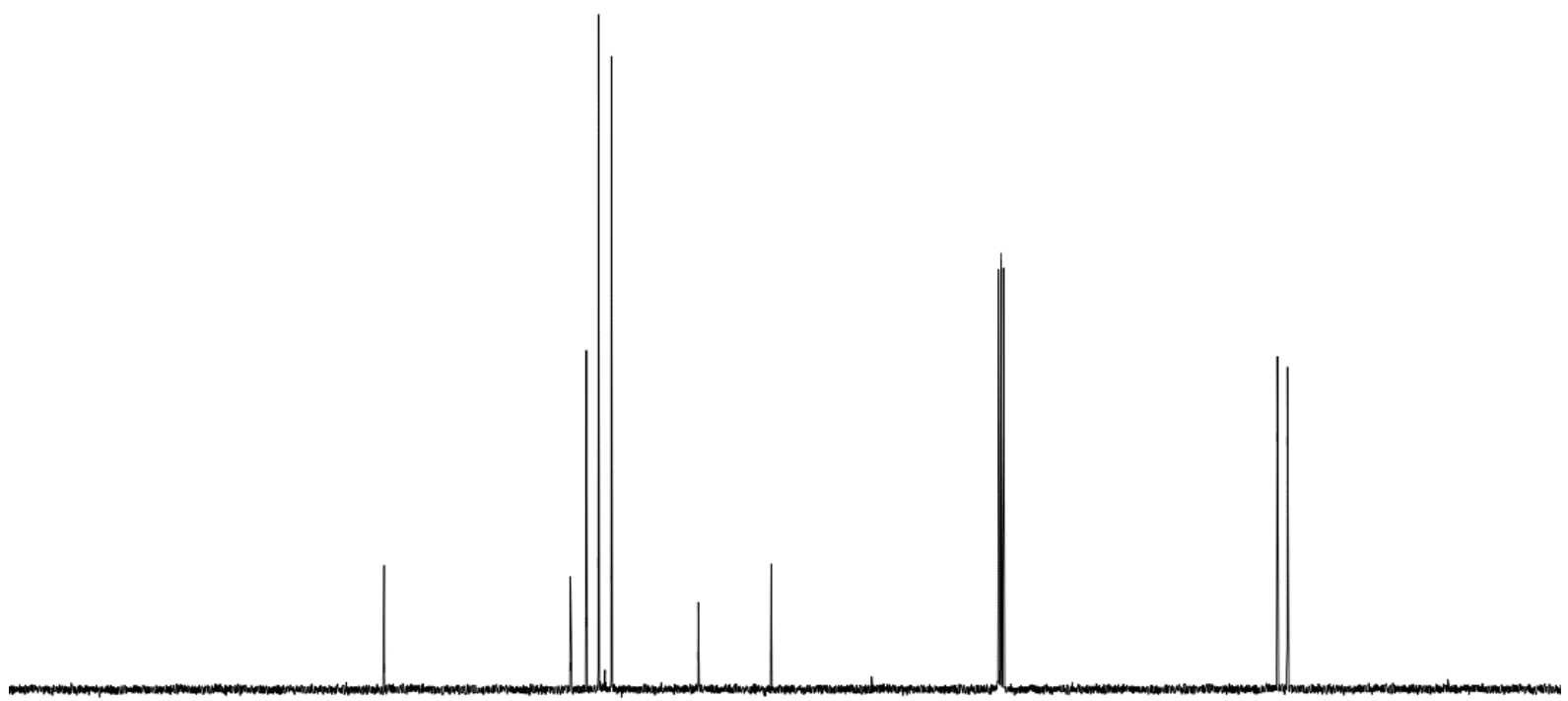

200 $\begin{array}{lllllllll}190 & 180 & 170 & 160 & 150 & 140 & 130 & 120 & \underset{\mathrm{f} 1(\mathrm{ppm})}{110}\end{array}$ 
<smiles>CCOC(=O)C1=C(c2ccccc2)CSC1</smiles>

$12 \mathrm{c}$

${ }^{1} \mathrm{H}$ NMR $\left(\mathrm{CDCl}_{3}, 400 \mathrm{MHz}\right)$

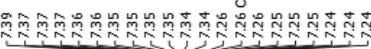

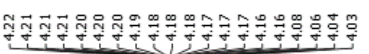

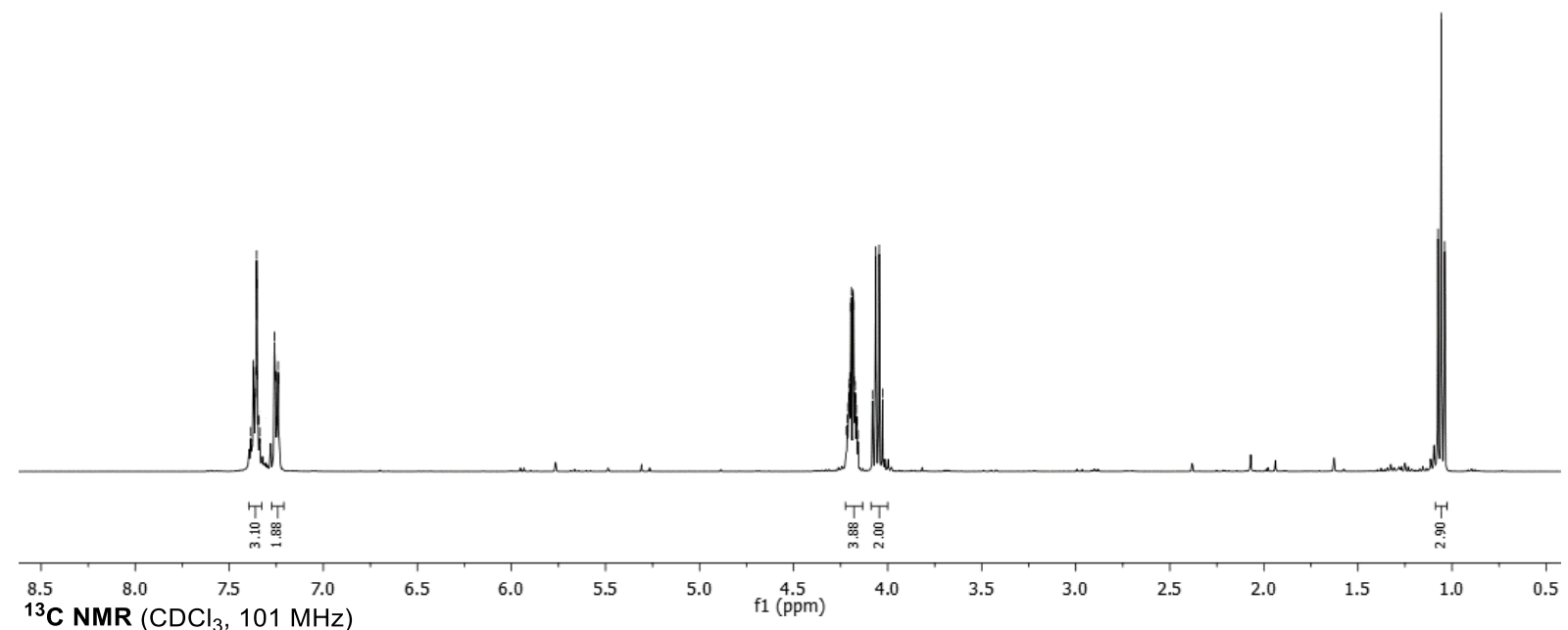

${ }^{13} \mathrm{C}$ NMR $\left(\mathrm{CDCl}_{3}, 101 \mathrm{MHz}\right)$
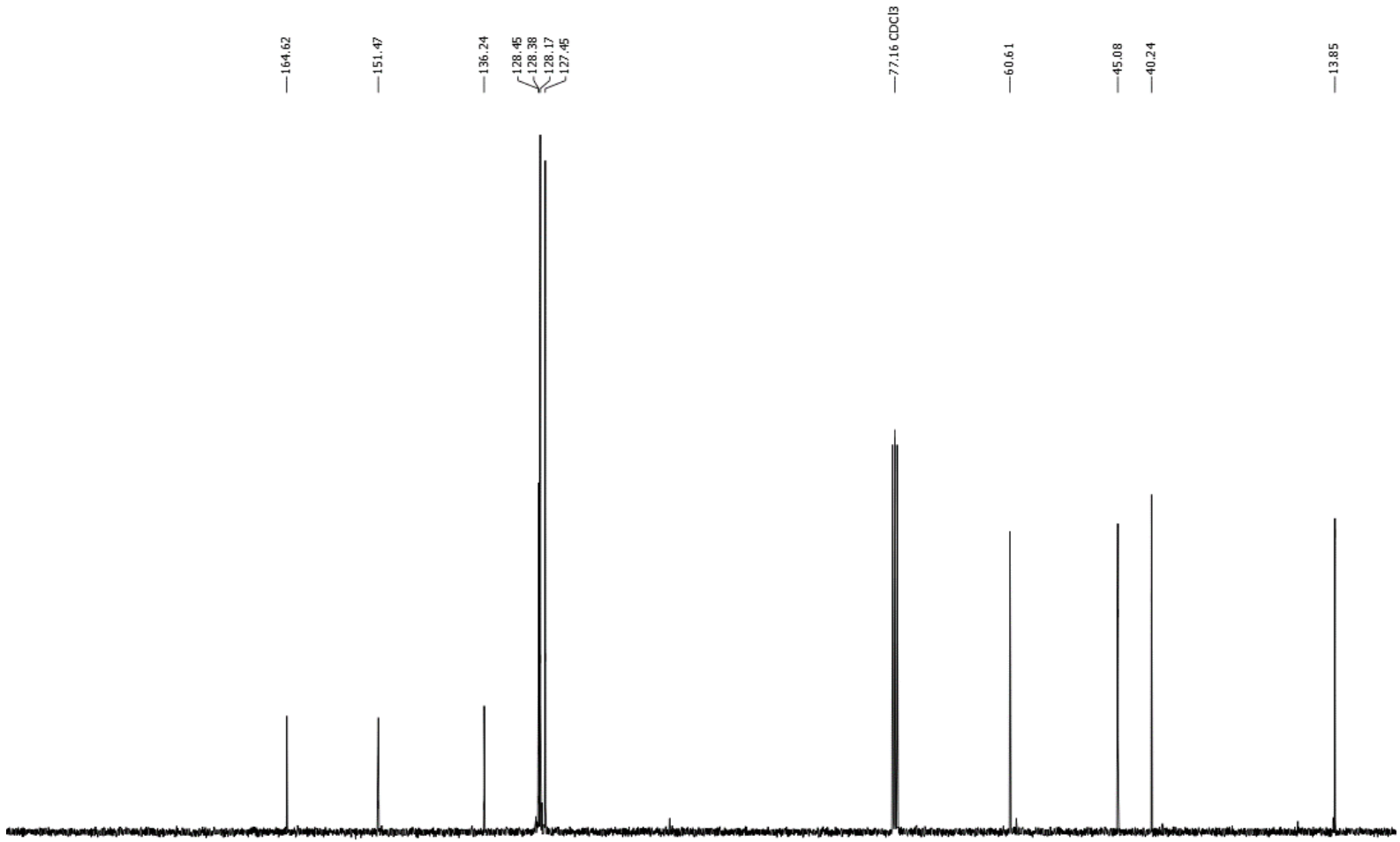
<smiles>CCC1=C(C(=O)OCc2ccccc2)CSC1</smiles>

12d

${ }^{1} \mathrm{H}$ NMR $\left(\mathrm{CDCl}_{3}, 400 \mathrm{MHz}\right)$

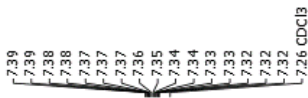
爱

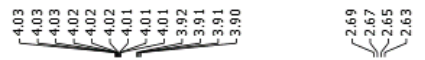

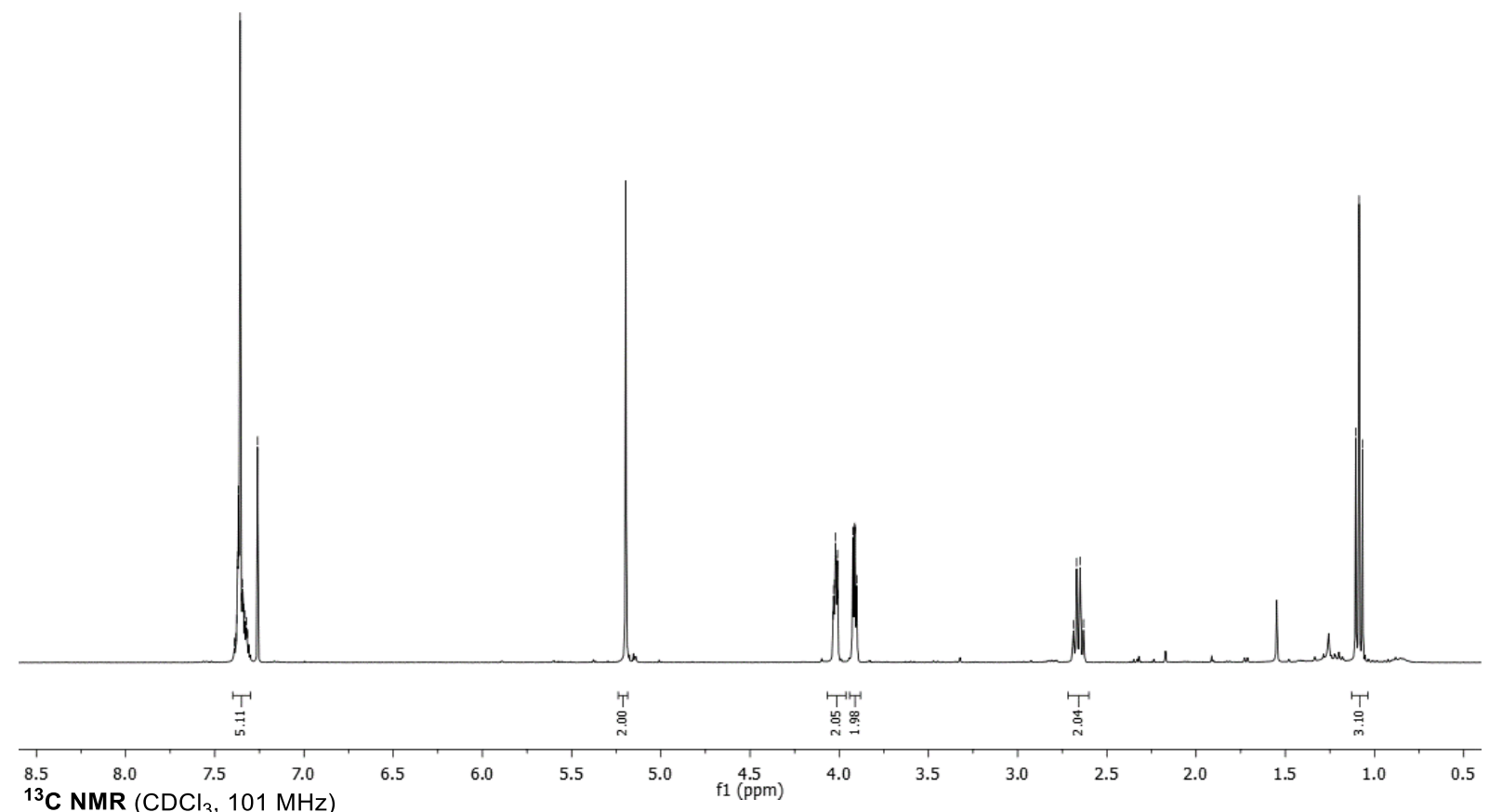

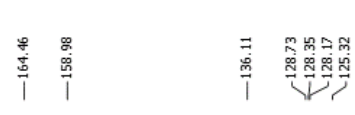

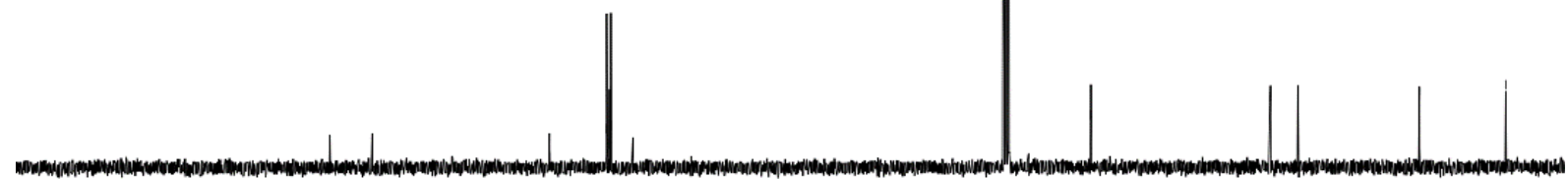




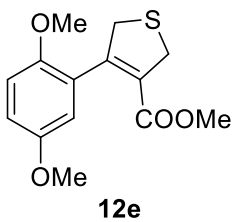

${ }^{1} \mathrm{H}$ NMR $\left(\mathrm{CDCl}_{3}, 400 \mathrm{MHz}\right)$

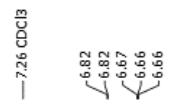

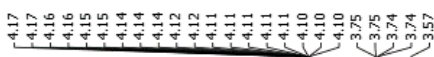
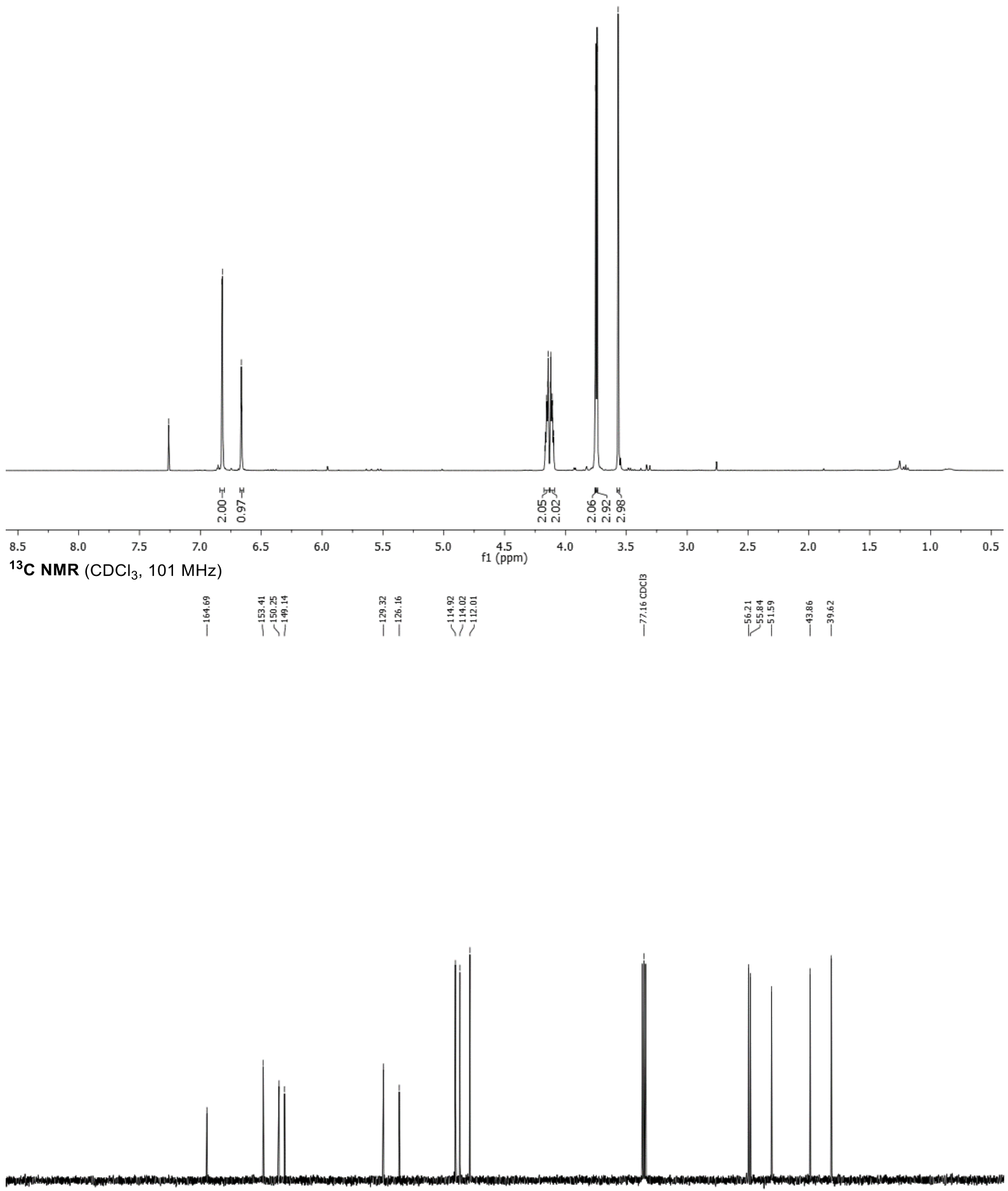

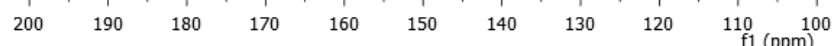


<smiles>COc1cscc1S(=O)(=O)[O-]</smiles>

$12 f$

${ }^{1} \mathrm{H}$ NMR $\left(\mathrm{CDCl}_{3}, 400 \mathrm{MHz}\right)$ the

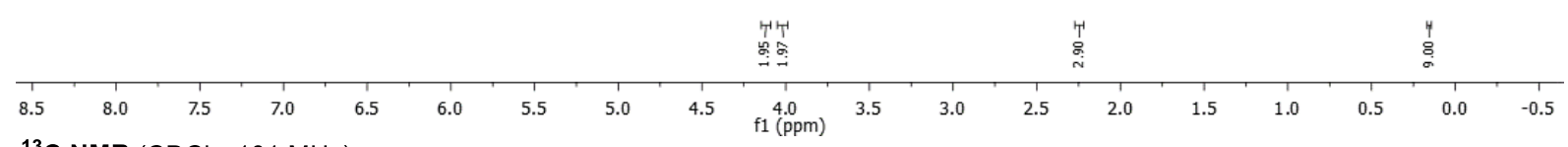

${ }^{13} \mathrm{C}$ NMR $\left(\mathrm{CDCl}_{3}, 101 \mathrm{MHz}\right)$

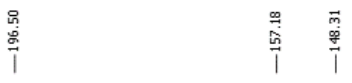


<smiles>CSC[C@@]1(C)CSC[C@@]1(C)C(C)=O</smiles>

$12 \mathrm{~g}$

${ }^{1} \mathrm{H}$ NMR $\left(\mathrm{CDCl}_{3}, 400 \mathrm{MHz}\right)$

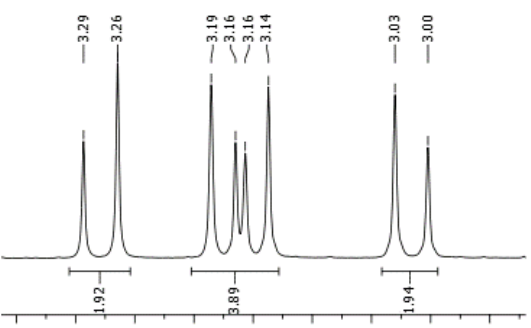

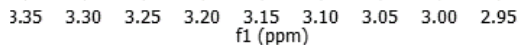

$\stackrel{+}{i}$

$\stackrel{0}{10}$

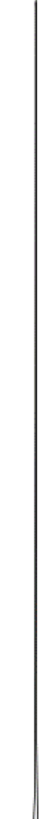

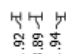

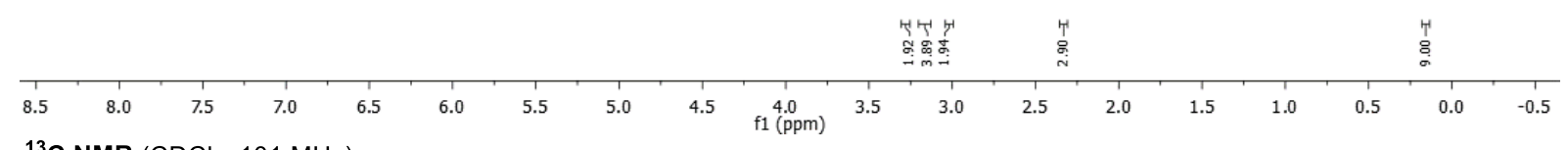

${ }^{13} \mathrm{C} \mathrm{NMR}\left(\mathrm{CDCl}_{3}, 101 \mathrm{MHz}\right)$

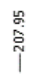

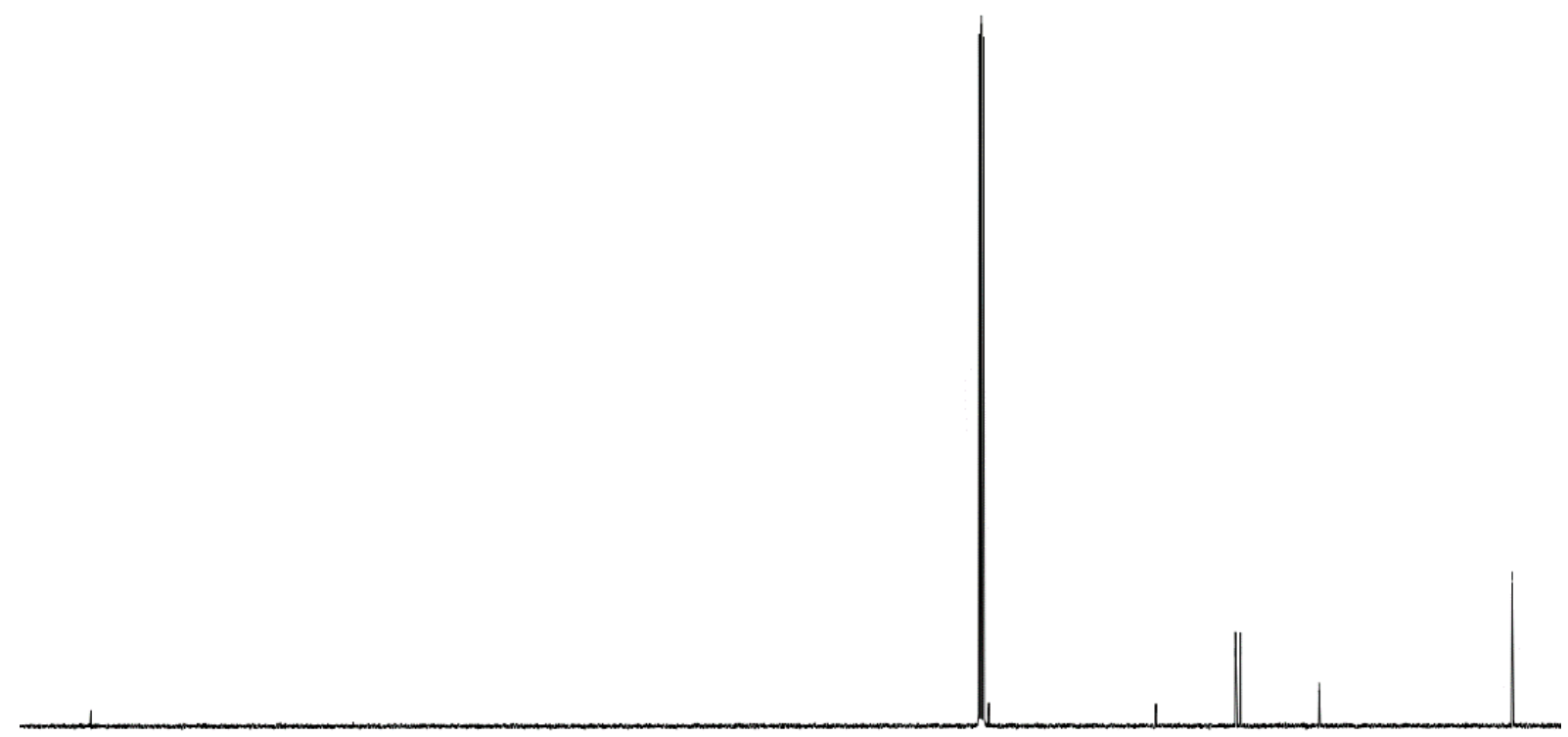

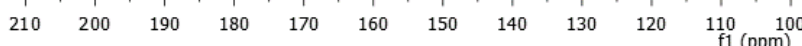




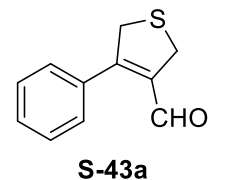

${ }^{1} \mathbf{H}$ NMR $\left(\mathrm{CDCl}_{3}, 400 \mathrm{MHz}\right)$

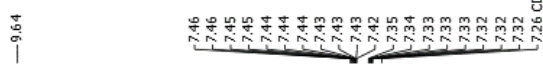
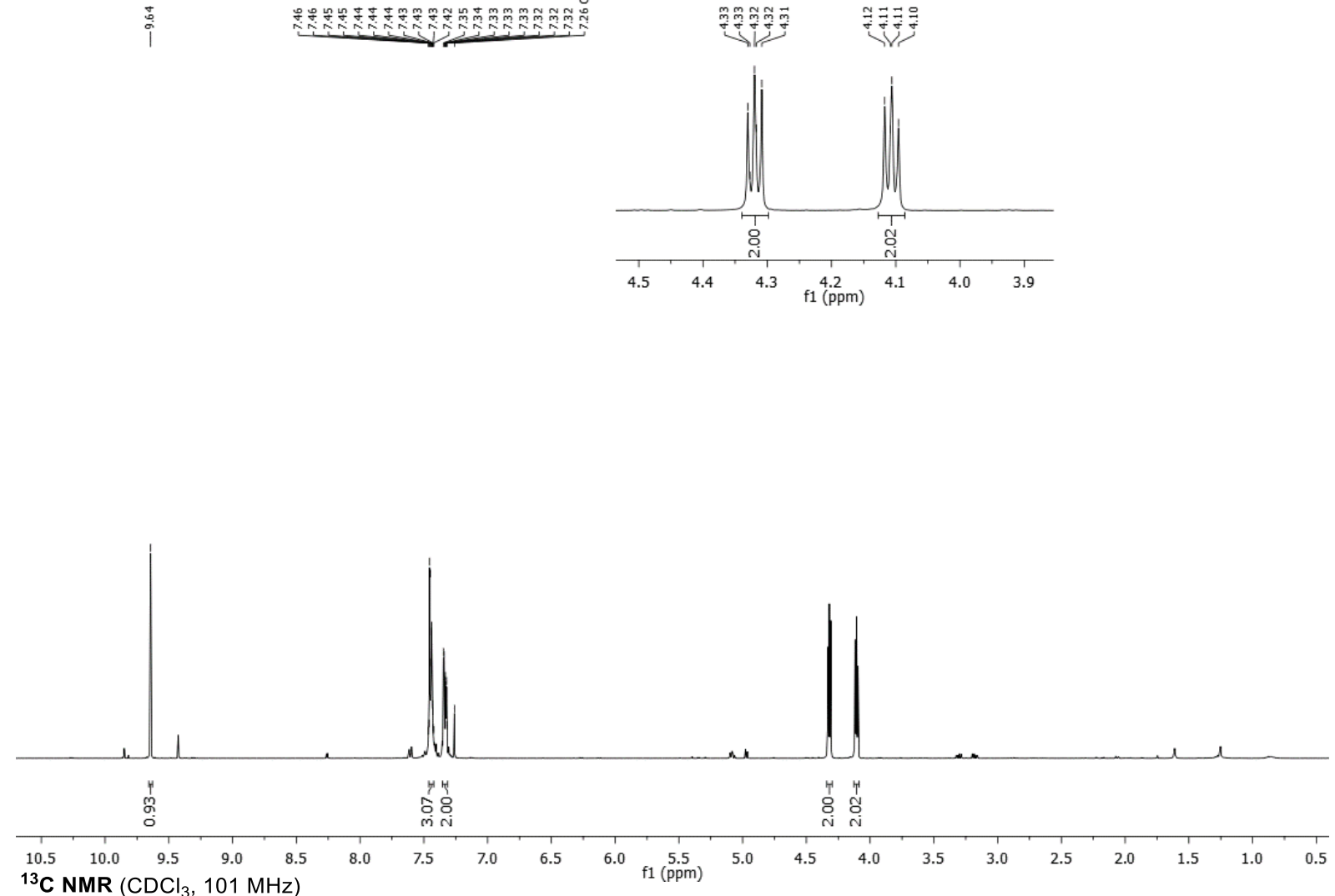

${ }^{13} \mathrm{C} \mathrm{NMR}\left(\mathrm{CDCl}_{3}, 101 \mathrm{MHz}\right)$
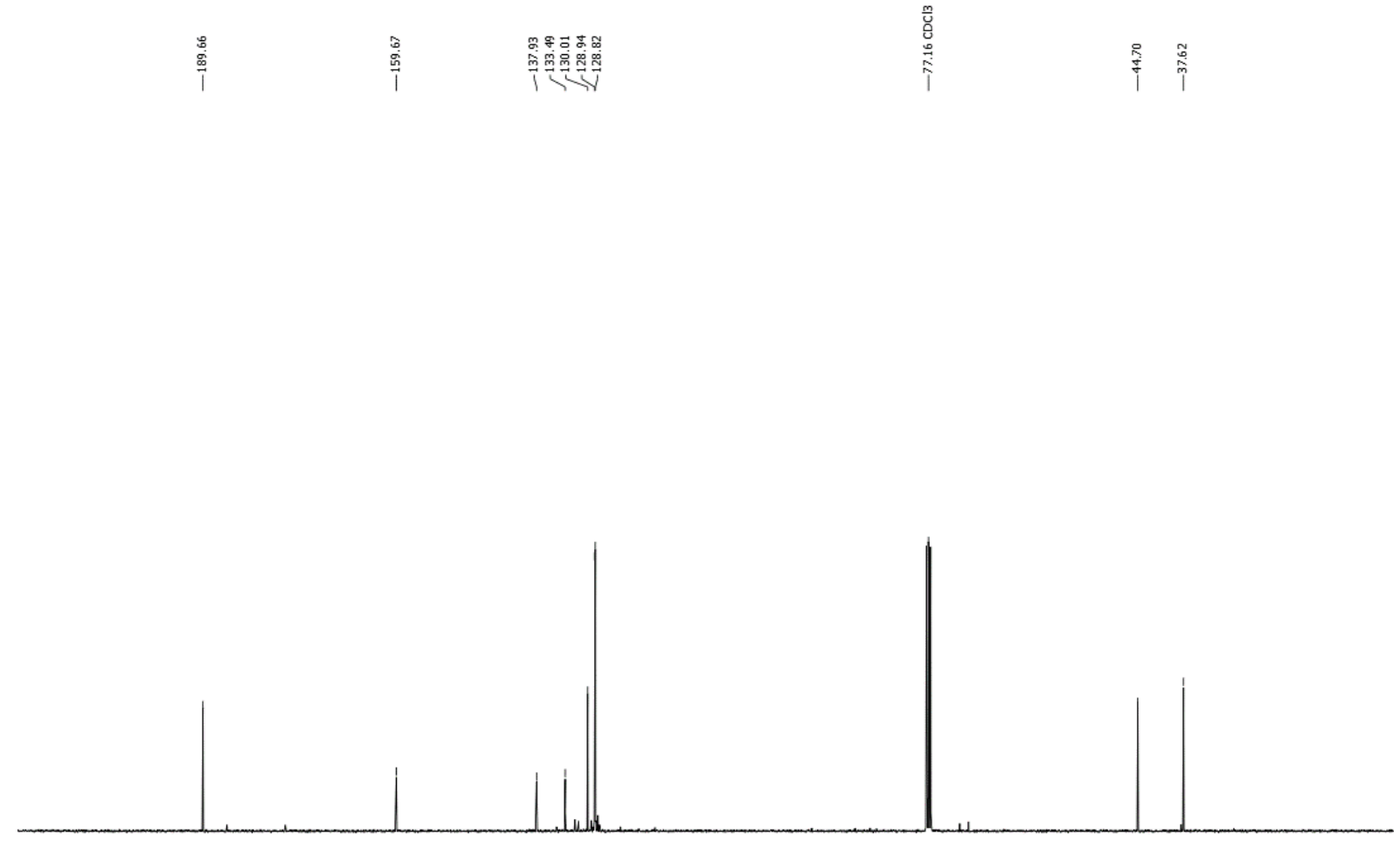

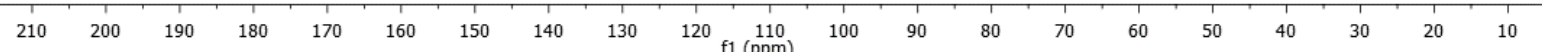




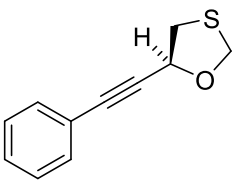

$\mathrm{S}-43 \mathrm{~b}$

${ }^{1} \mathbf{H}$ NMR $\left(\mathrm{CDCl}_{3}, 400 \mathrm{MHz}\right)$

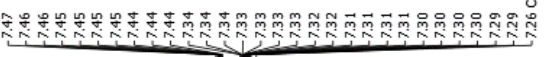
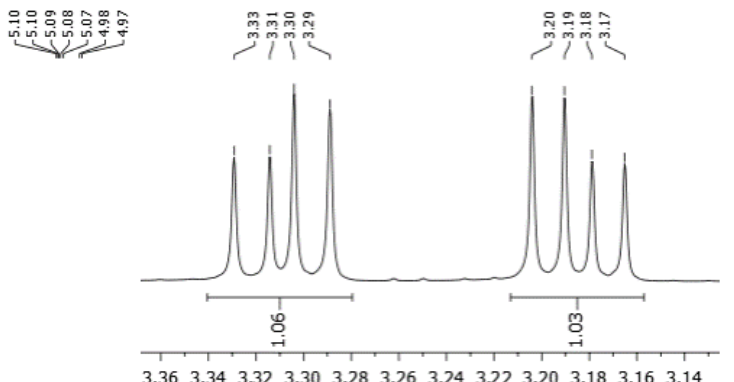

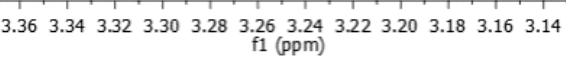

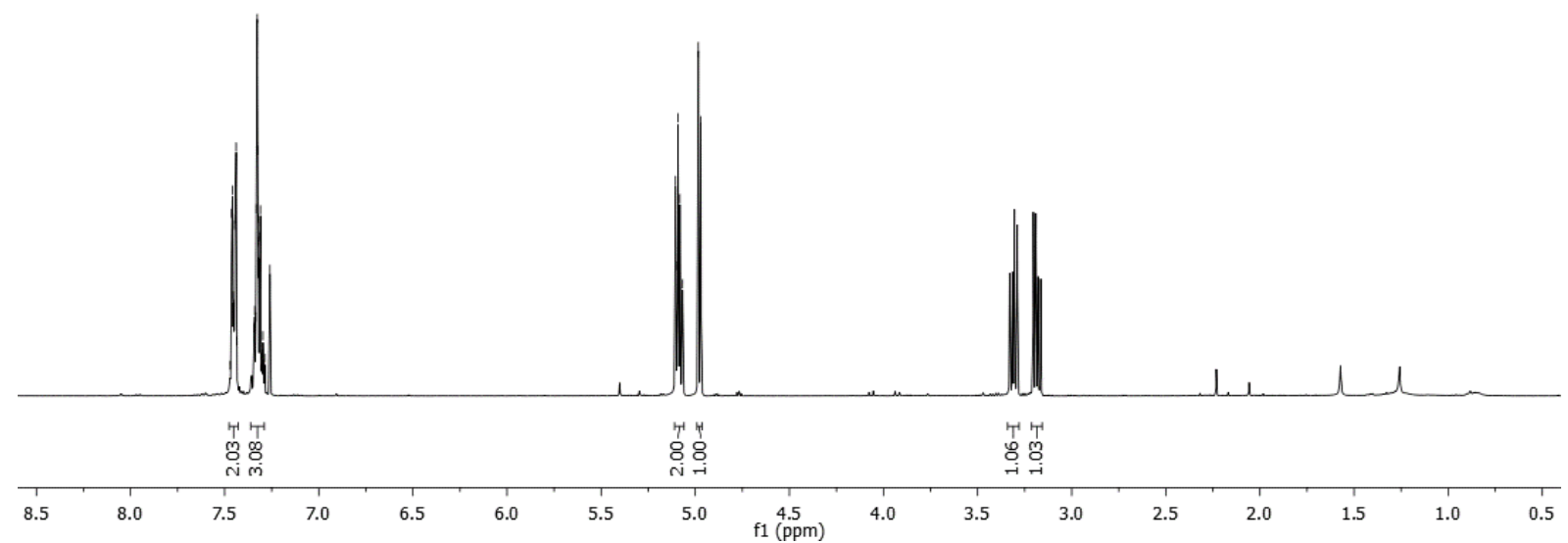

${ }^{13} \mathrm{C}$ NMR $\left(\mathrm{CDCl}_{3}, 101 \mathrm{MHz}\right)$

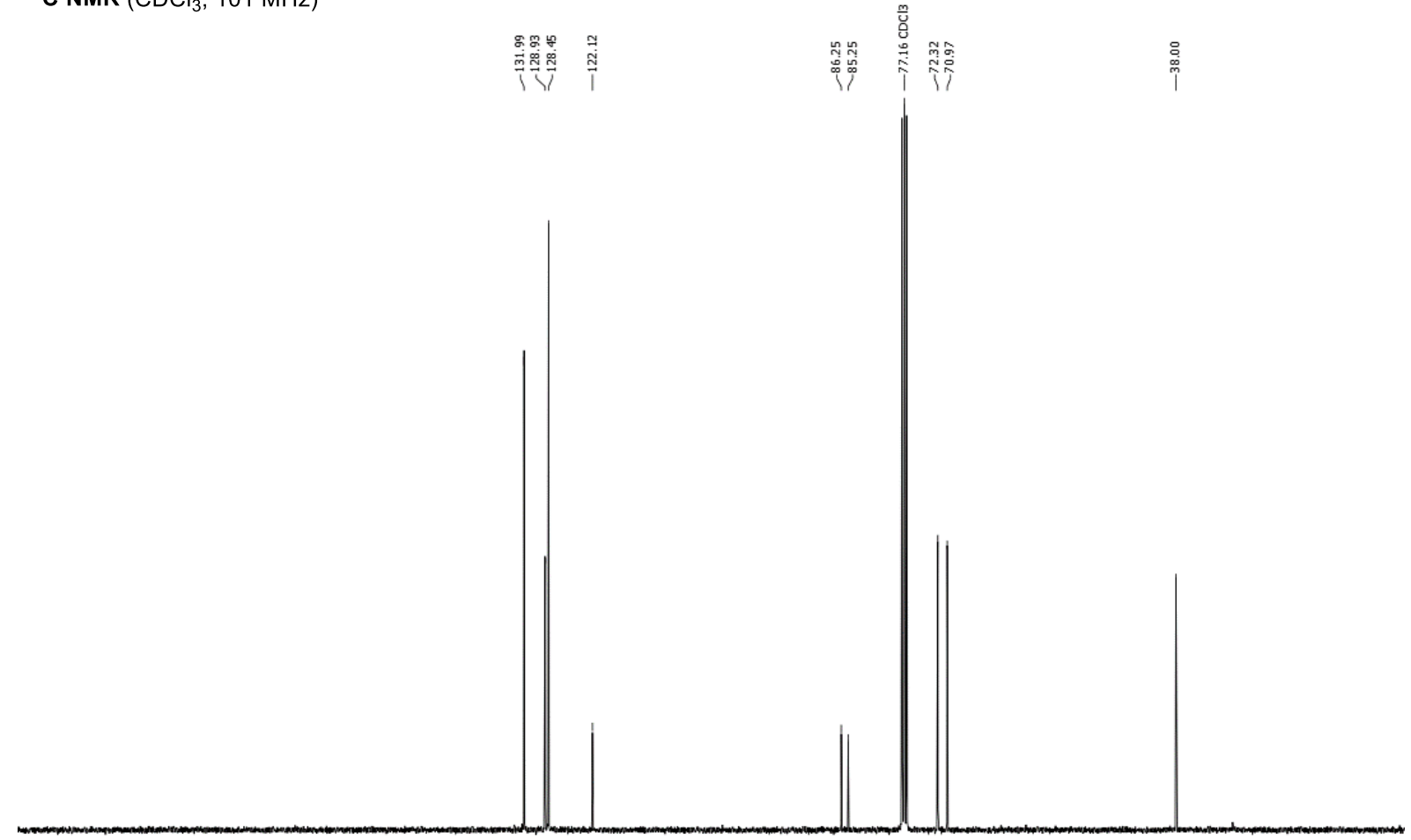

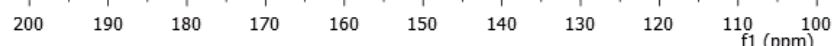


<smiles>c1ccc(OCC2CCSC2)cc1</smiles>

S-44

${ }^{1} \mathrm{H} \mathrm{NMR}\left(\mathrm{CDCl}_{3}, 400 \mathrm{MHz}\right)$

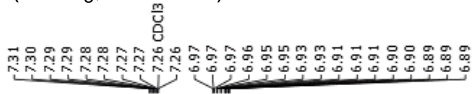

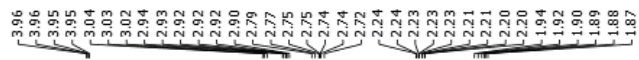

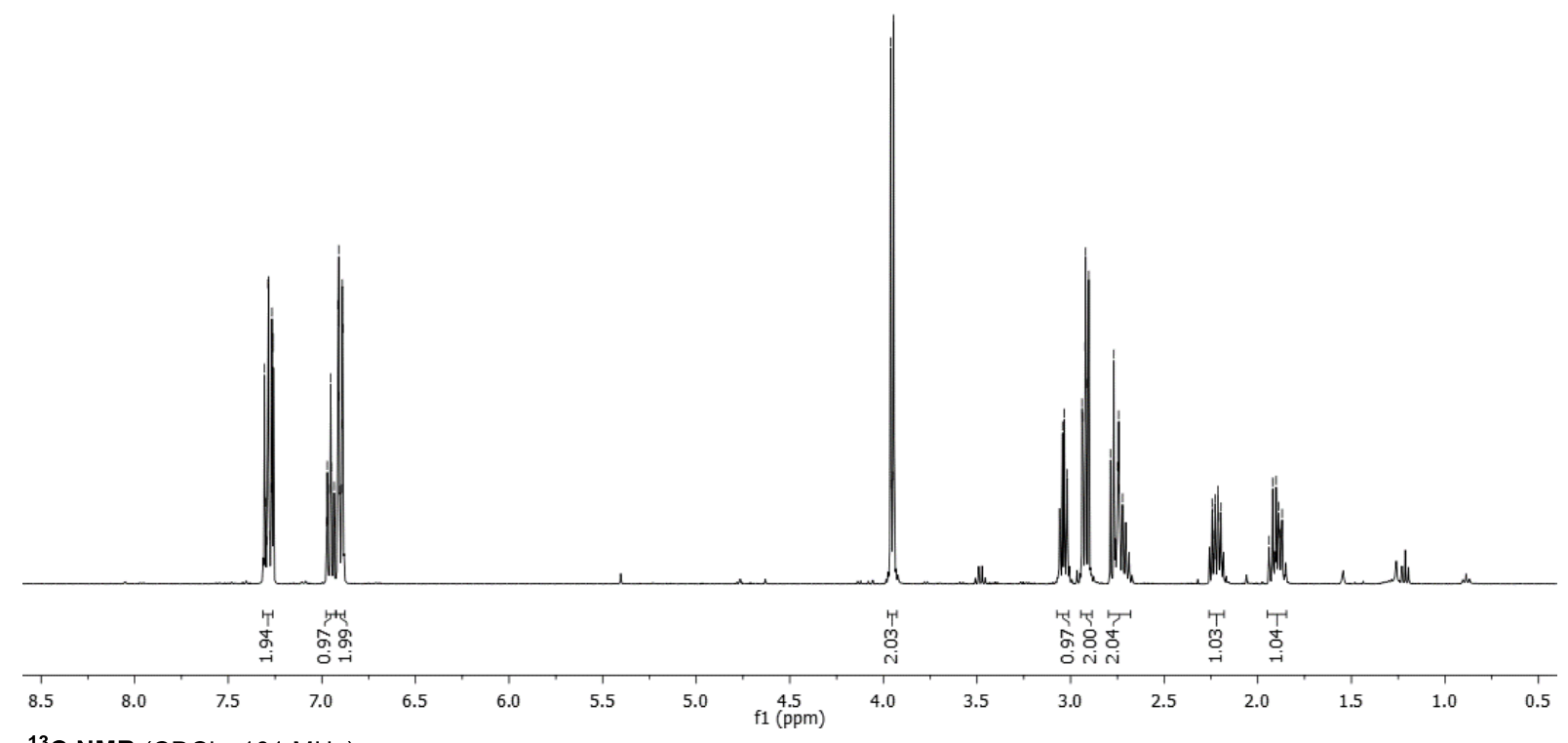

${ }^{13} \mathrm{C}$ NMR $\left(\mathrm{CDCl}_{3}, 101 \mathrm{MHz}\right)$

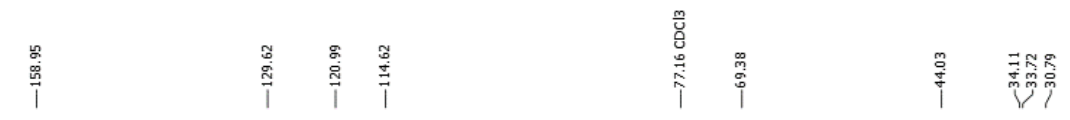

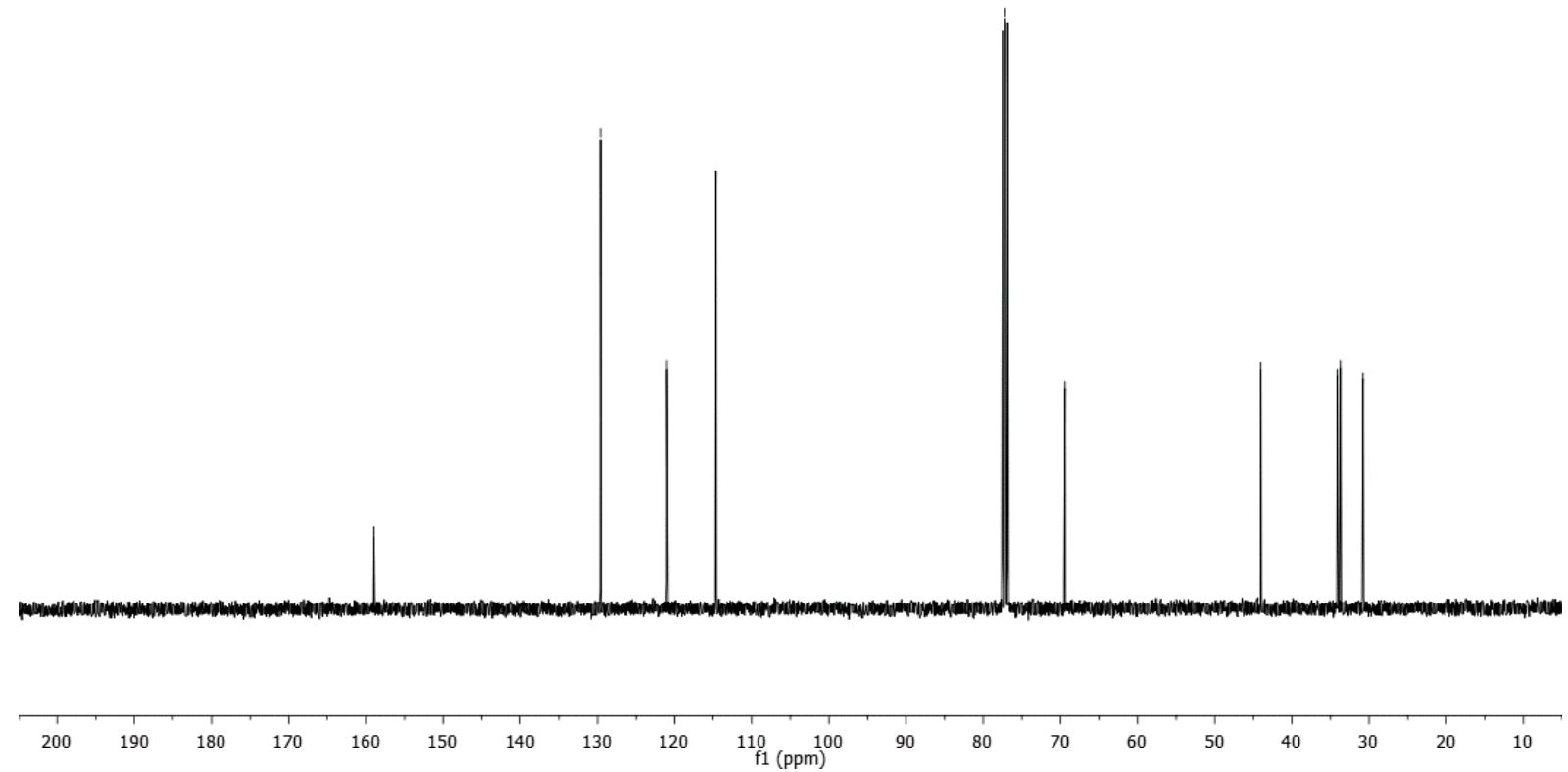




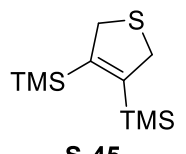

S-45

${ }^{1} \mathrm{H}$ NMR $\left(\mathrm{CDCl}_{3}, 400 \mathrm{MHz}\right)$
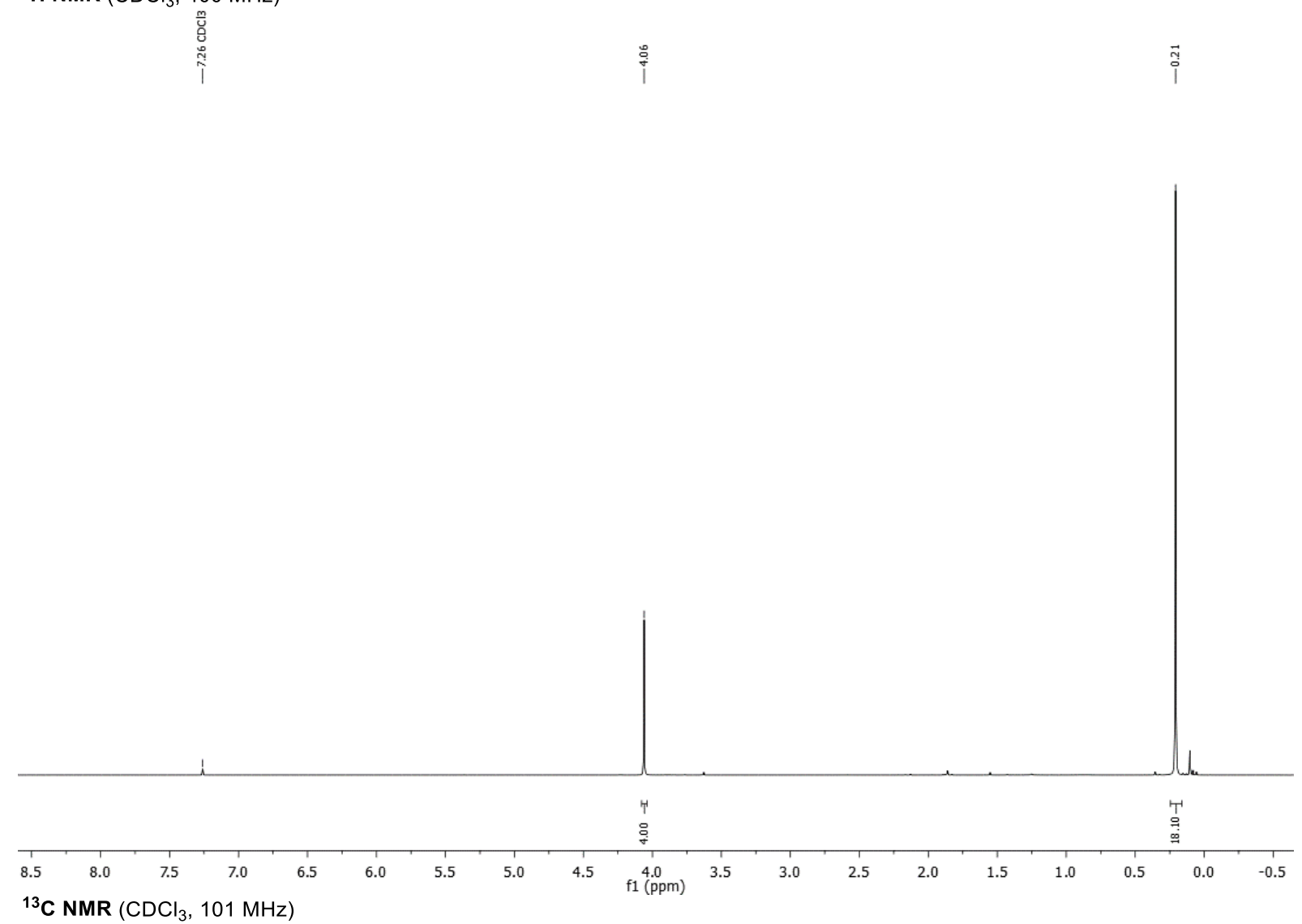

${ }^{13} \mathrm{C}$ NMR $\left(\mathrm{CDCl}_{3}, 101 \mathrm{MHz}\right)$
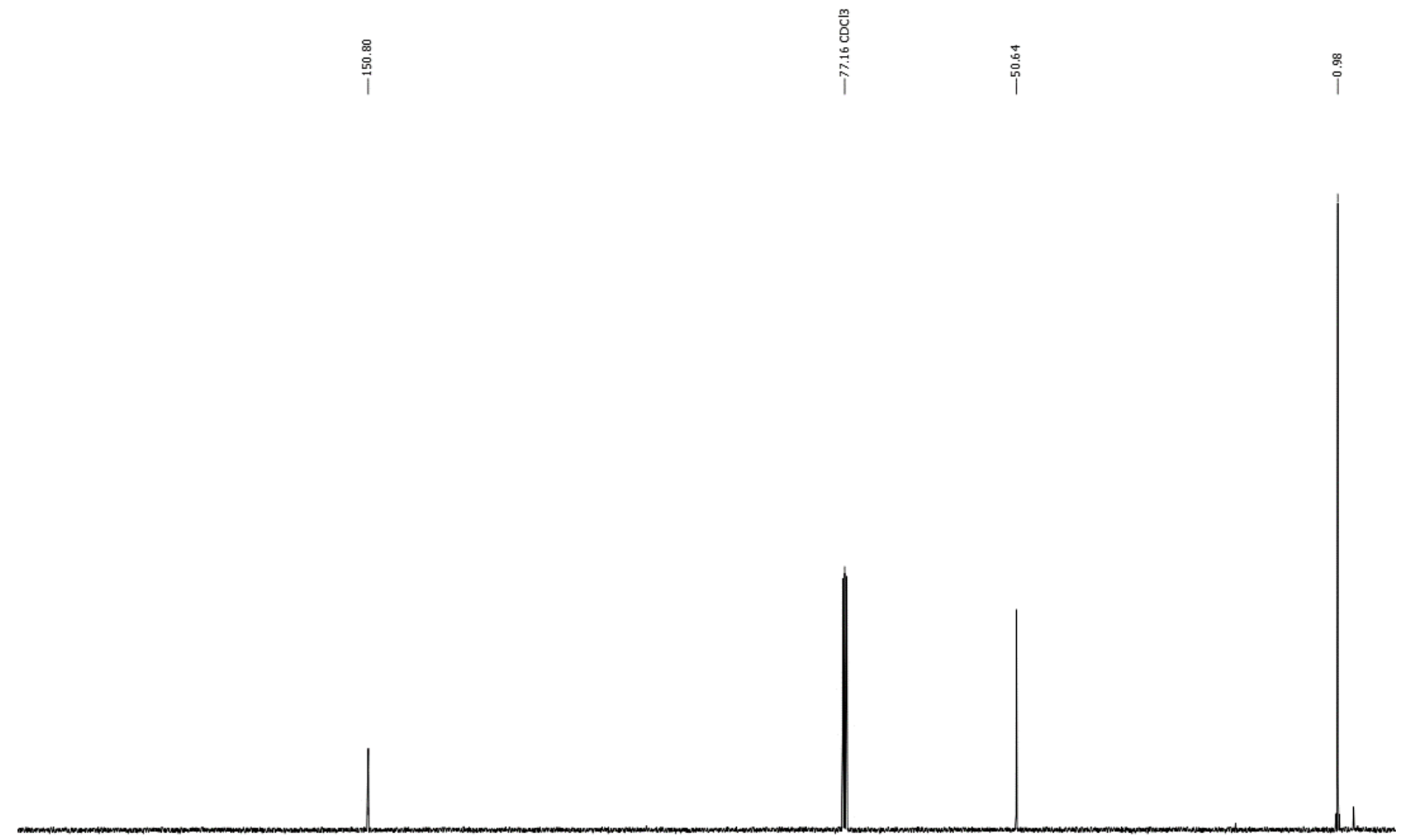

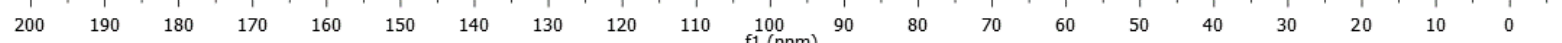




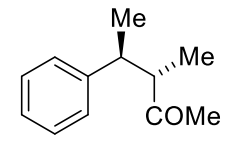

$13(\mathrm{dr}=5: 1)$

${ }^{1} \mathrm{H} \mathrm{NMR}\left(\mathrm{CDCl}_{3}, 400 \mathrm{MHz}\right)$

兽

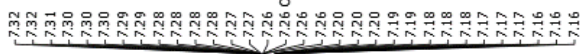
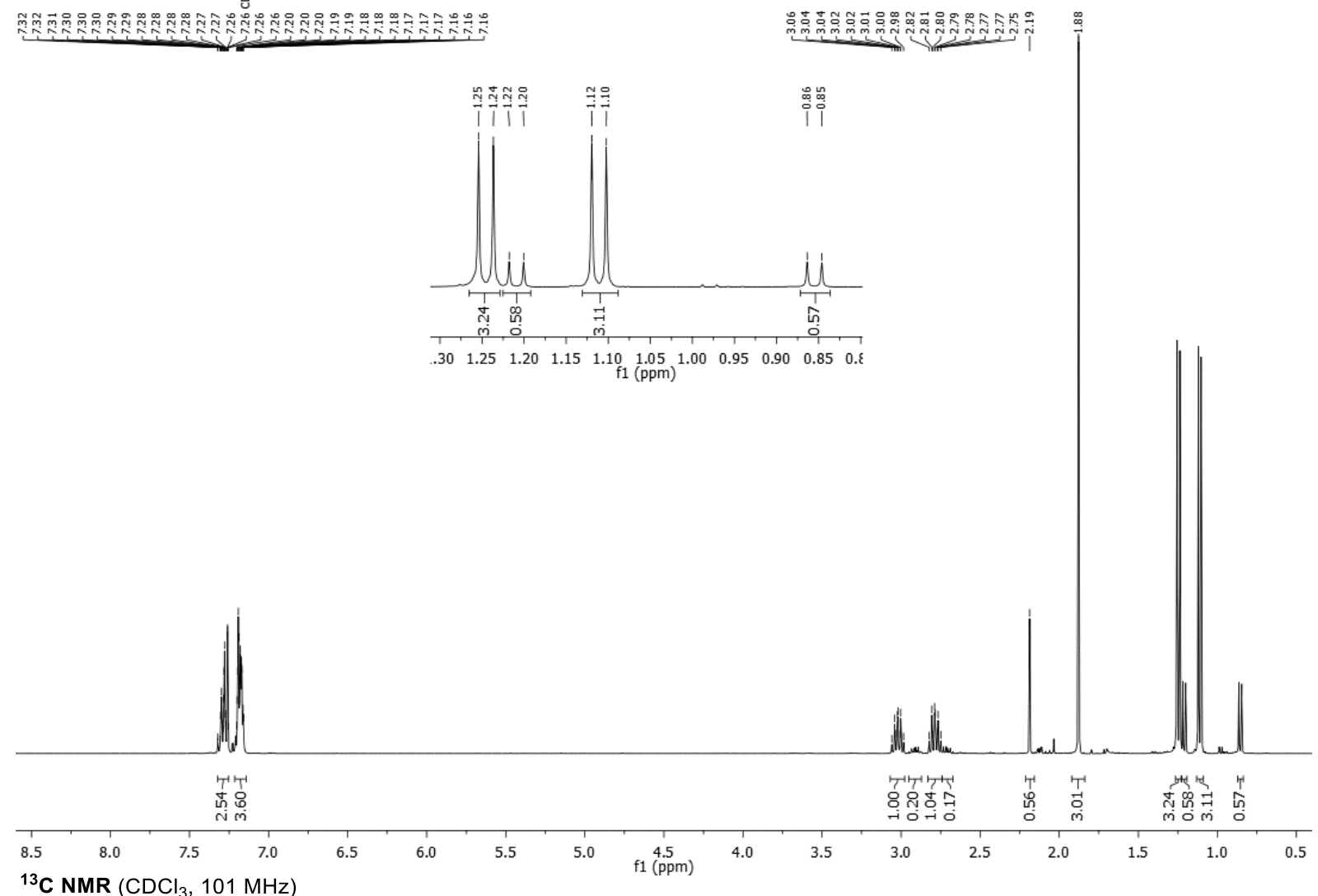

${ }^{13} \mathrm{C}$ NMR $\left(\mathrm{CDCl}_{3}, 101 \mathrm{MHz}\right)$

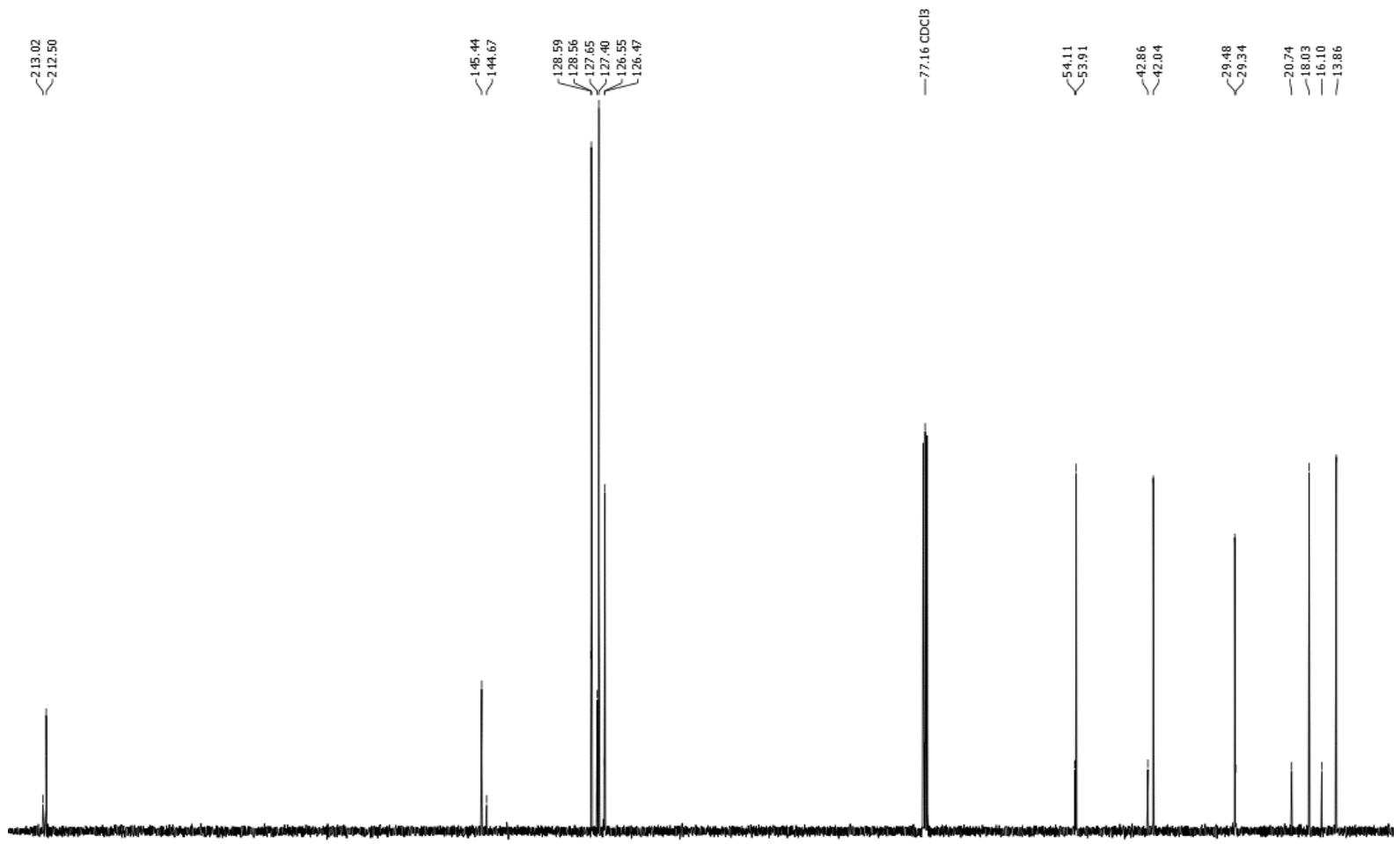

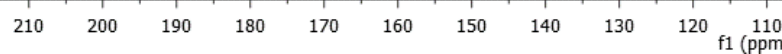




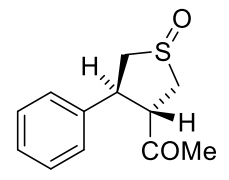

$14(\mathrm{dr}=1.3: 1)$

${ }^{1} \mathrm{H}$ NMR $\left(\mathrm{CDCl}_{3}, 400 \mathrm{MHz}\right)$

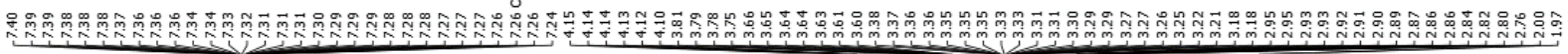

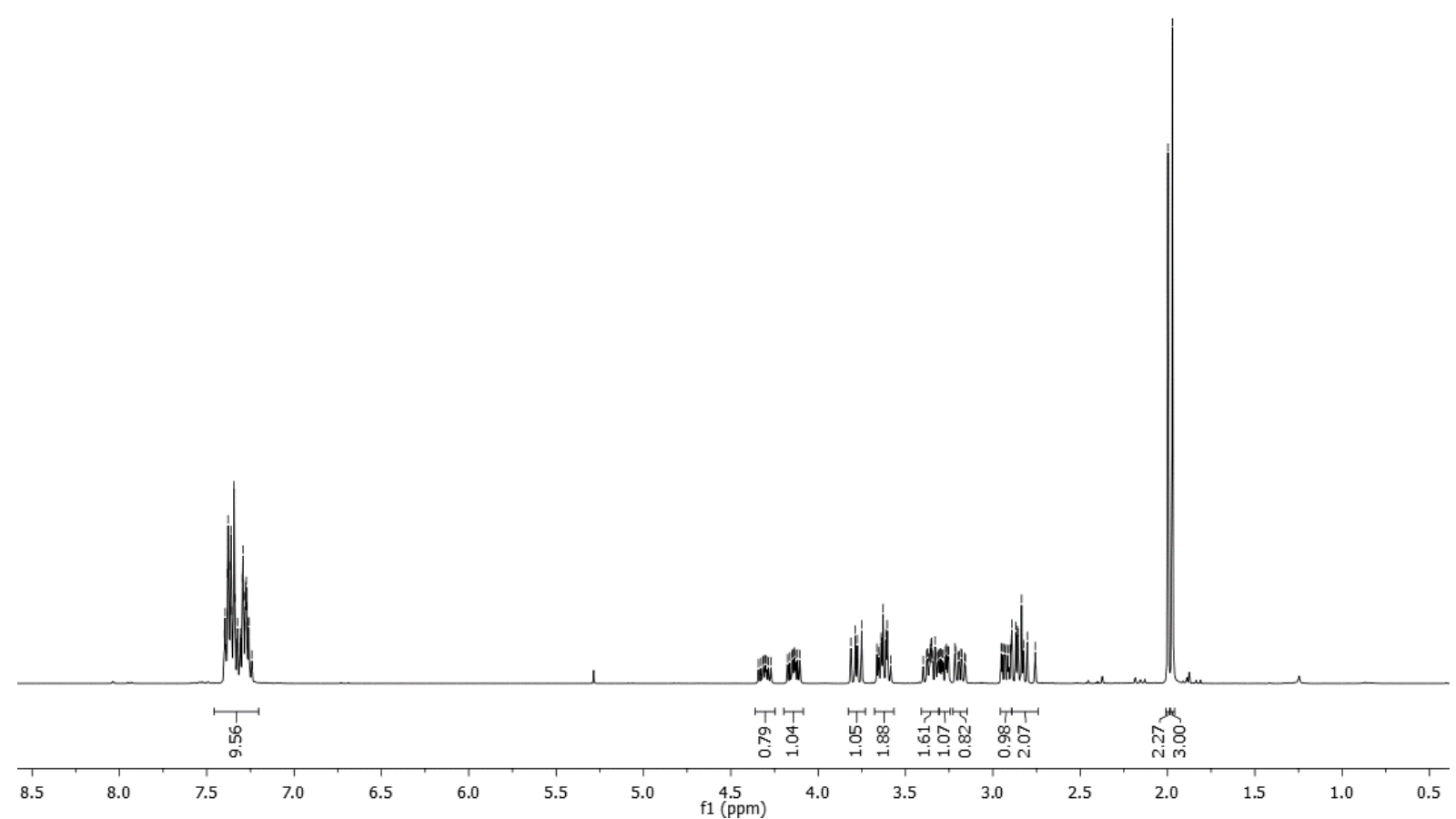

${ }^{13} \mathrm{C}$ NMR $\left(\mathrm{CDCl}_{3}, 101 \mathrm{MHz}\right)$

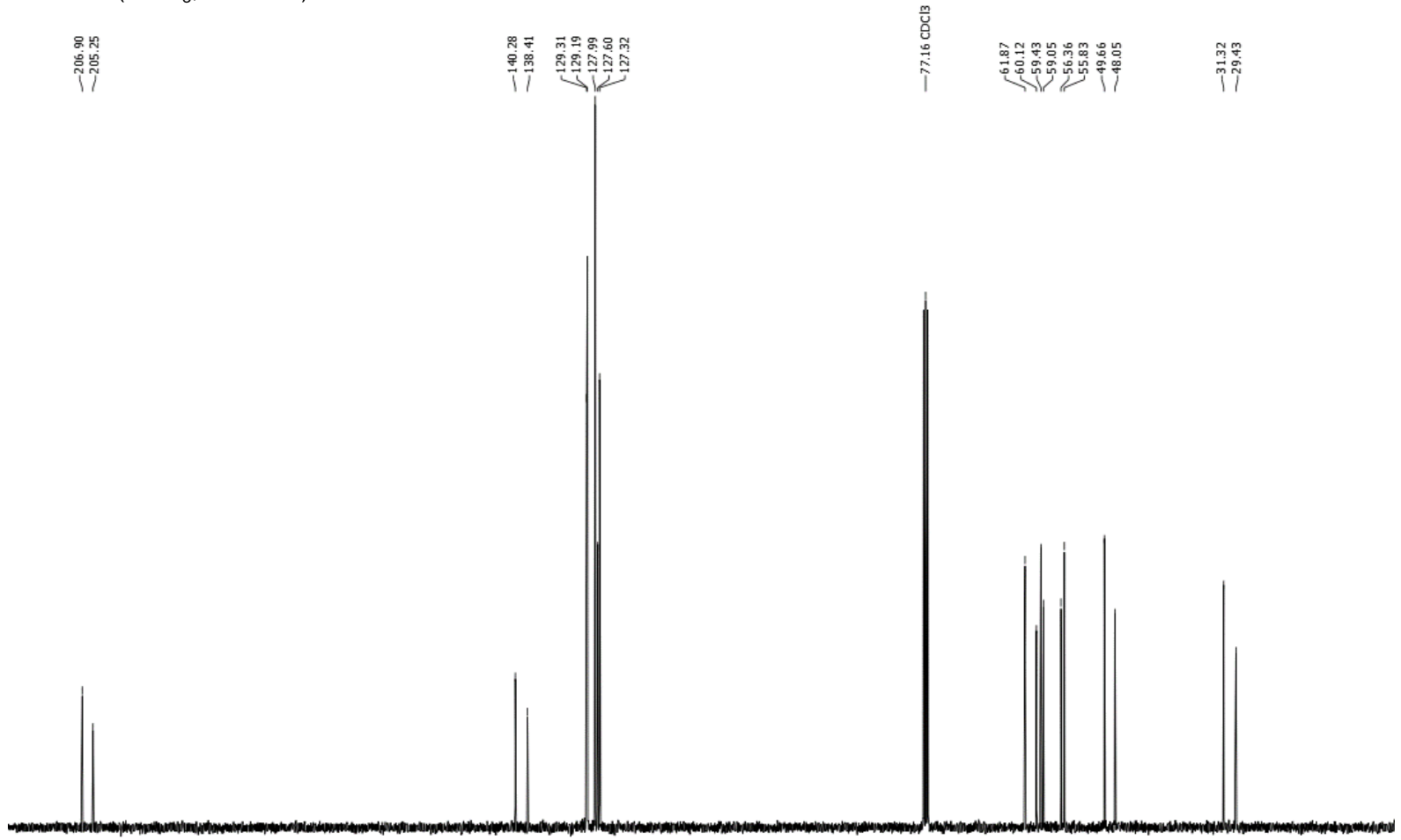

$\begin{array}{llllllllll}210 & 200 & 190 & 180 & 170 & 160 & 150 & 140 & 130 & 120 \\ \mathrm{f} 1(\mathrm{ppm})\end{array}$ 


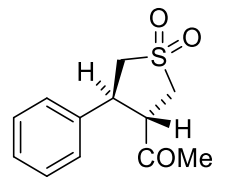

15

${ }^{1} \mathrm{H} \mathrm{NMR}\left(\mathrm{CDCl}_{3}, 400 \mathrm{MHz}\right)$

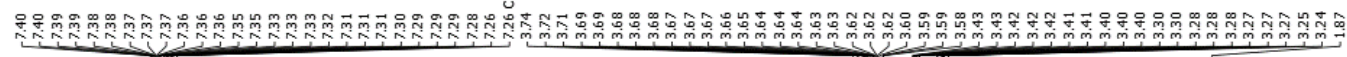

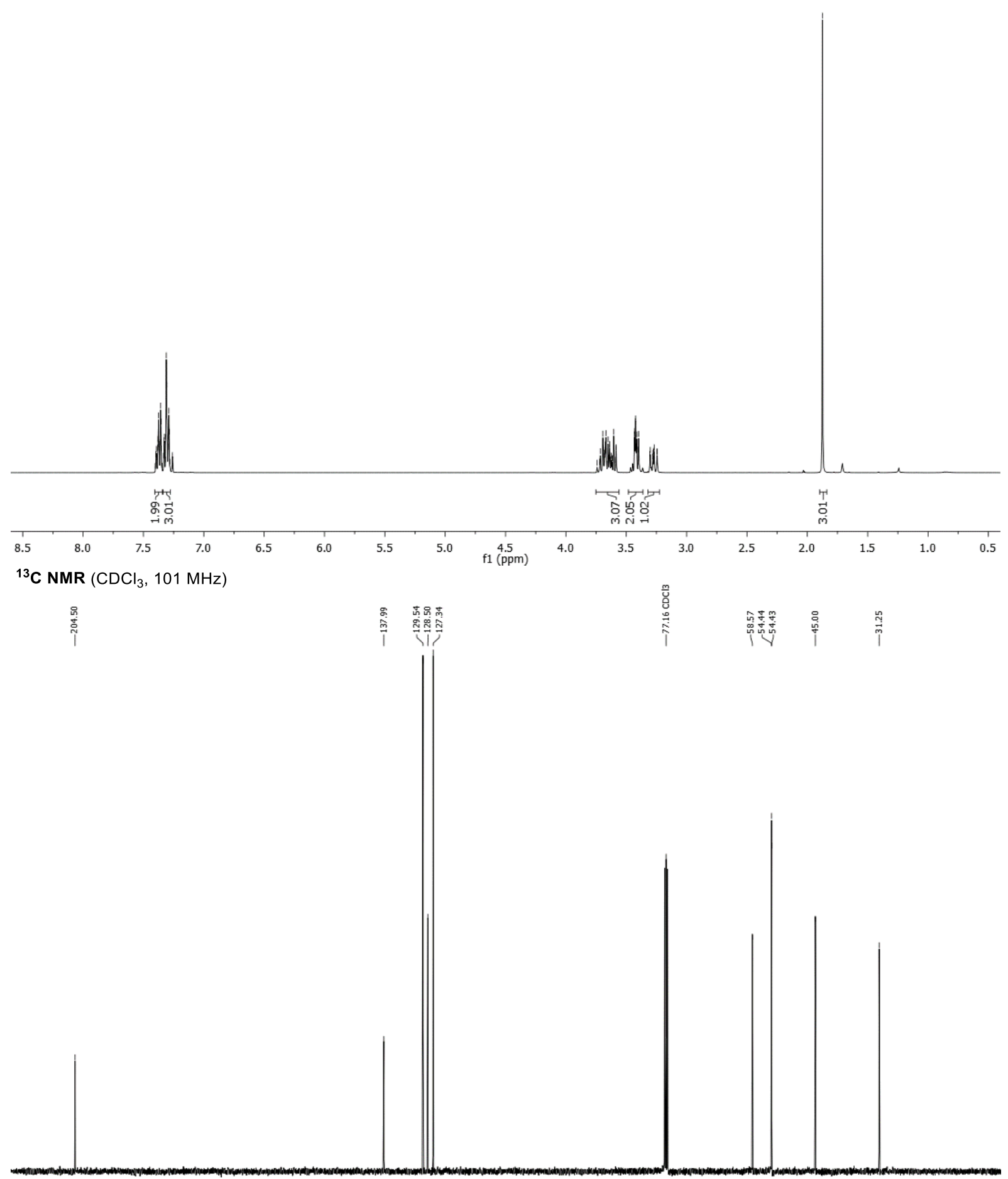

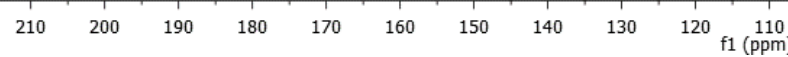




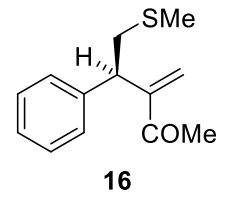

${ }^{1} \mathrm{H}$ NMR $\left(\mathrm{CDCl}_{3}, 400 \mathrm{MHz}\right)$

营

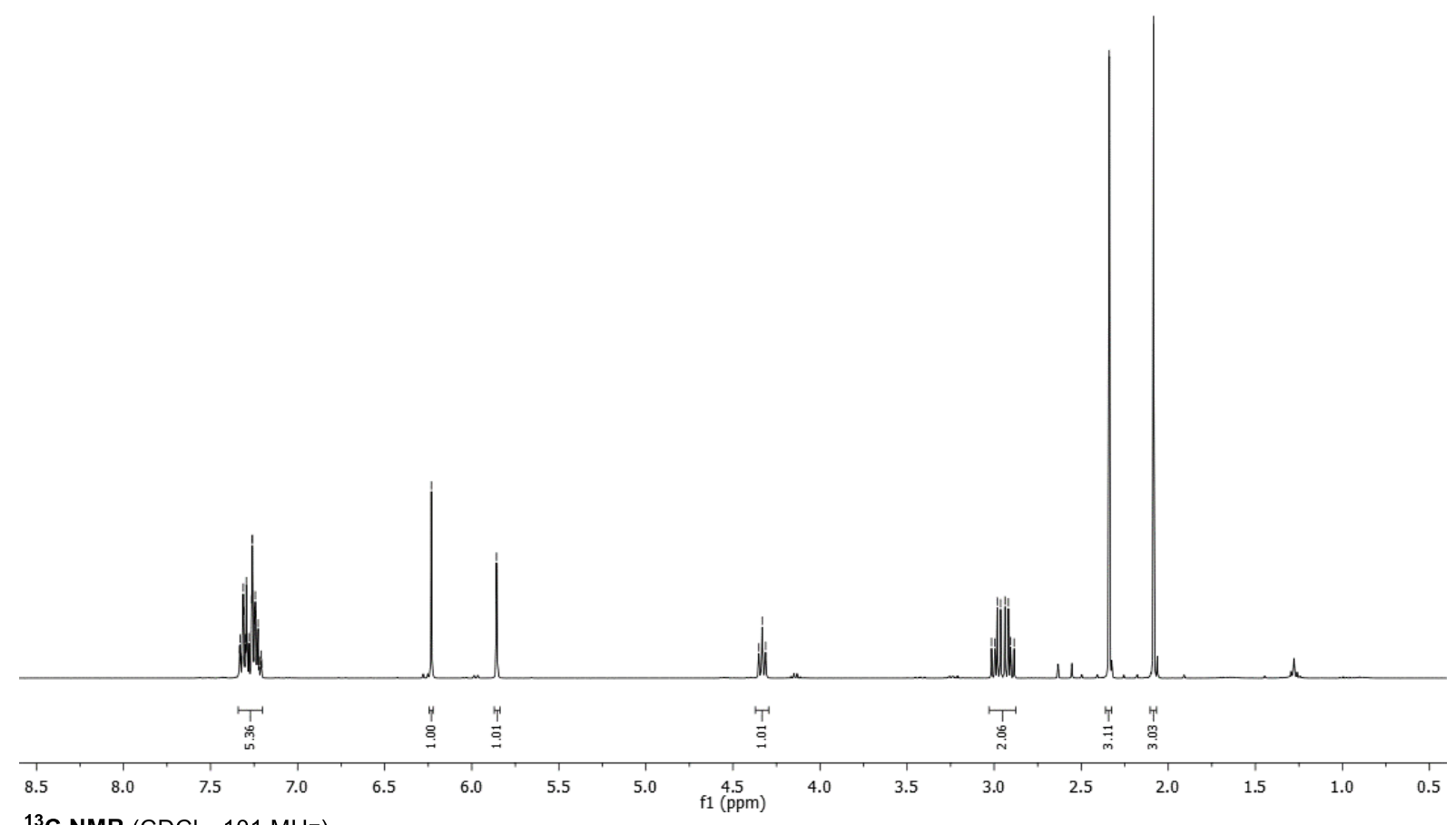

${ }^{13} \mathrm{C}$ NMR $\left(\mathrm{CDCl}_{3}, 101 \mathrm{MHz}\right)$

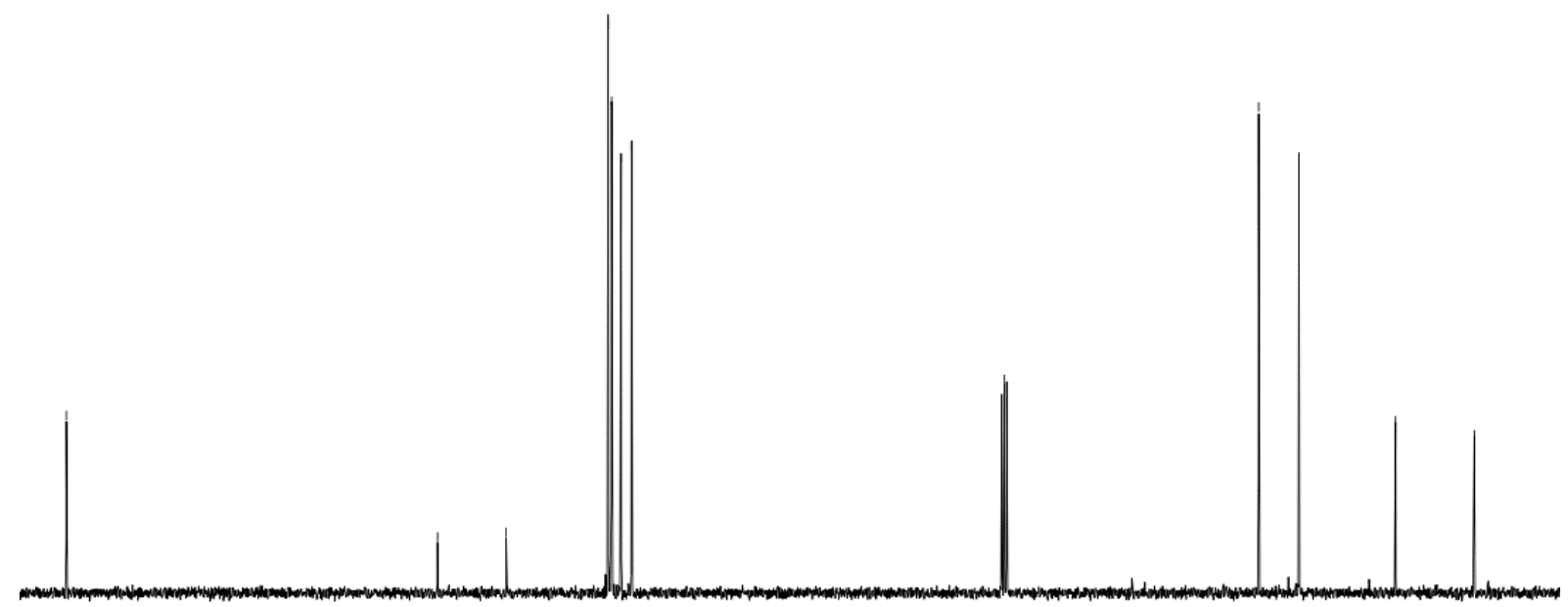




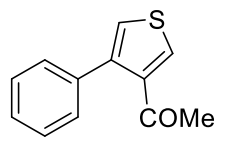

17

${ }^{1} \mathrm{H}$ NMR $\left(\mathrm{CDCl}_{3}, 400 \mathrm{MHz}\right)$<smiles>[B]=C1CC1</smiles>
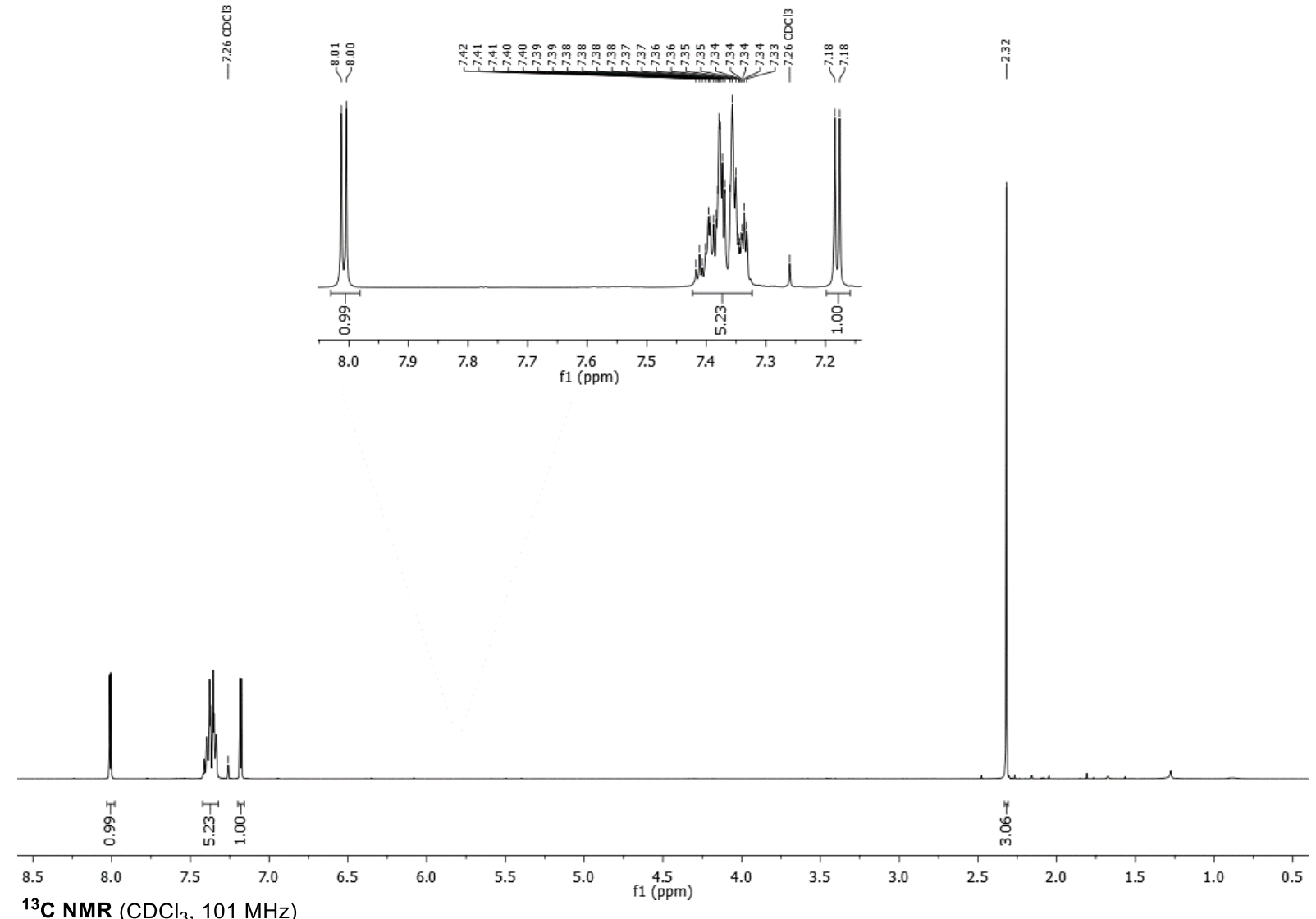

${ }^{13} \mathrm{C} \mathrm{NMR}\left(\mathrm{CDCl}_{3}, 101 \mathrm{MHz}\right)$

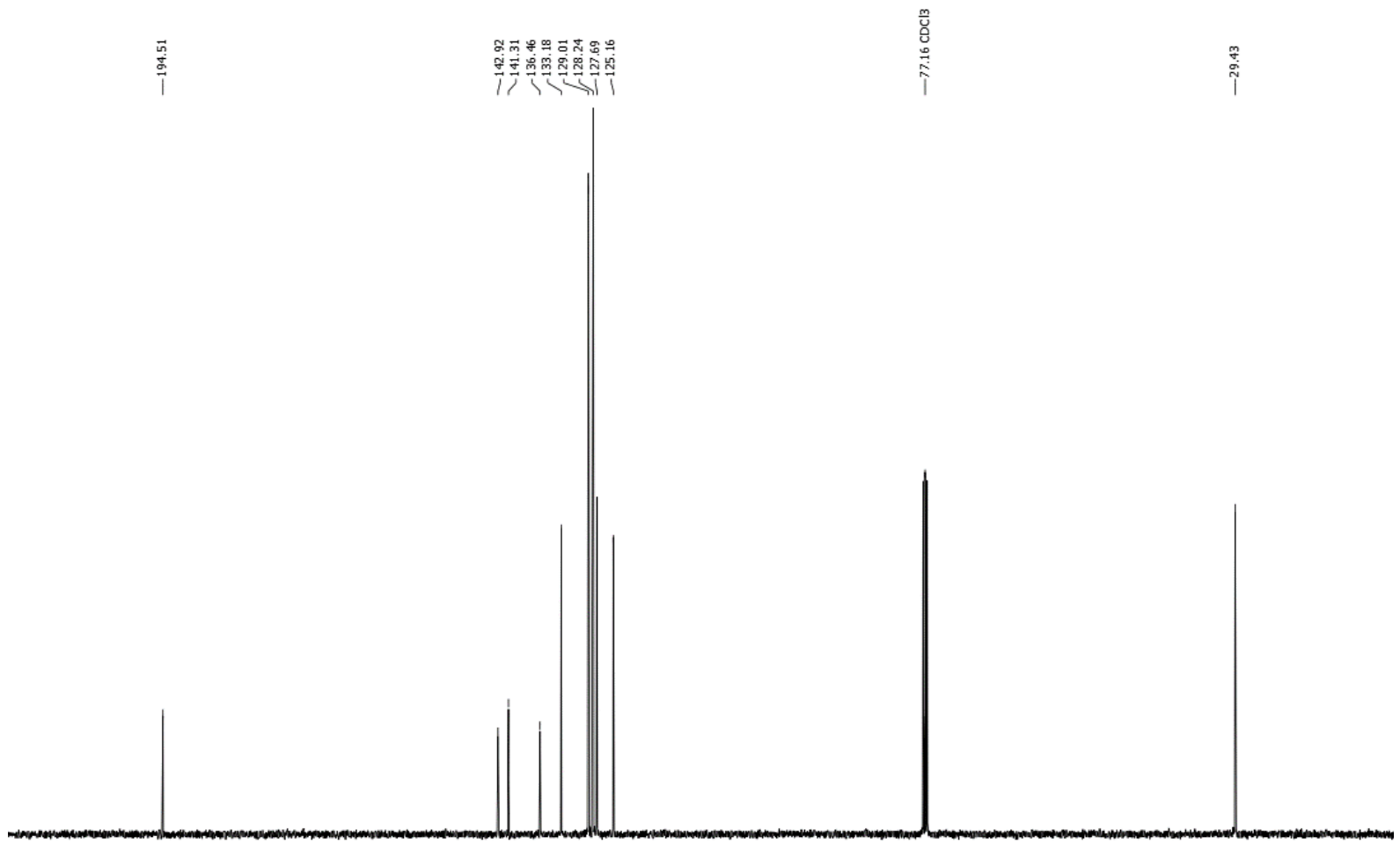

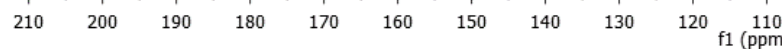


<smiles>CC(=O)OC1=C(c2ccccc2)CS(=O)(=O)C1</smiles>

18

${ }^{1} \mathrm{H}$ NMR $\left(\mathrm{CDCl}_{3}, 400 \mathrm{MHz}\right)$

営

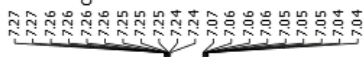

$\stackrel{i}{i}$

whe
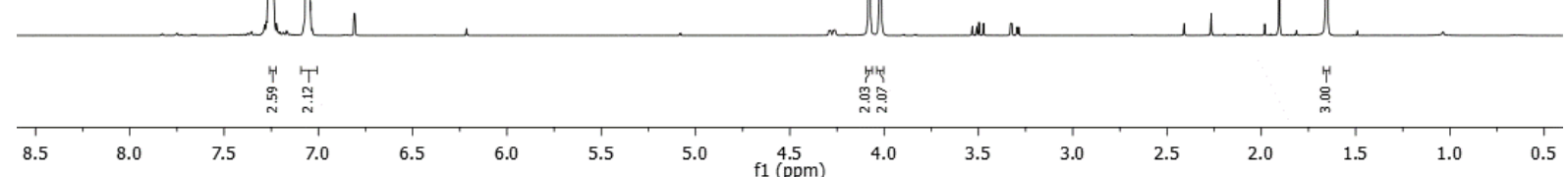

${ }^{13} \mathrm{C}$ NMR $\left(\mathrm{CDCl}_{3}, 101 \mathrm{MHz}\right)$

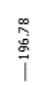

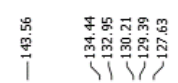

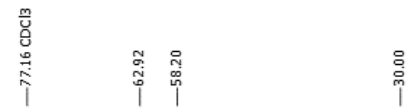

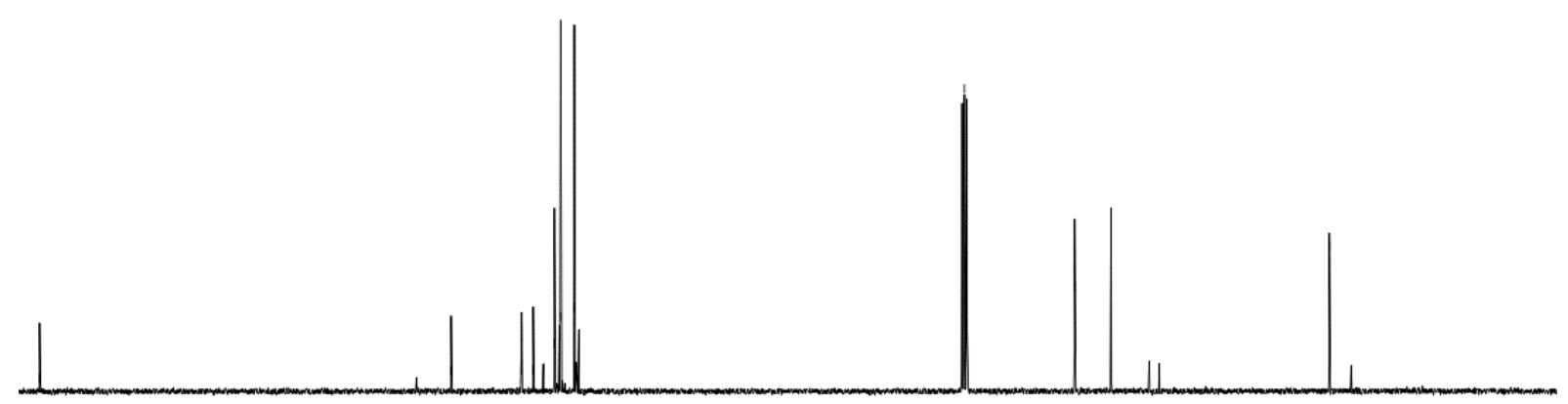

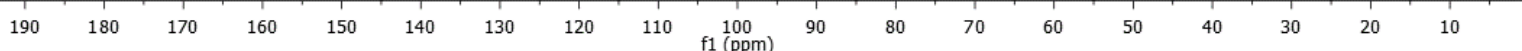


营

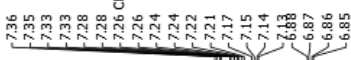<smiles>CC(=O)C1=C(c2ccccc2)C[C@H]2C(=O)NC(=O)C(Cc3ccccc3)[C@H]2C1</smiles>

20

${ }^{1} \mathrm{H}$ NMR $\left(\mathrm{CDCl}_{3}, 400 \mathrm{MHz}\right)$

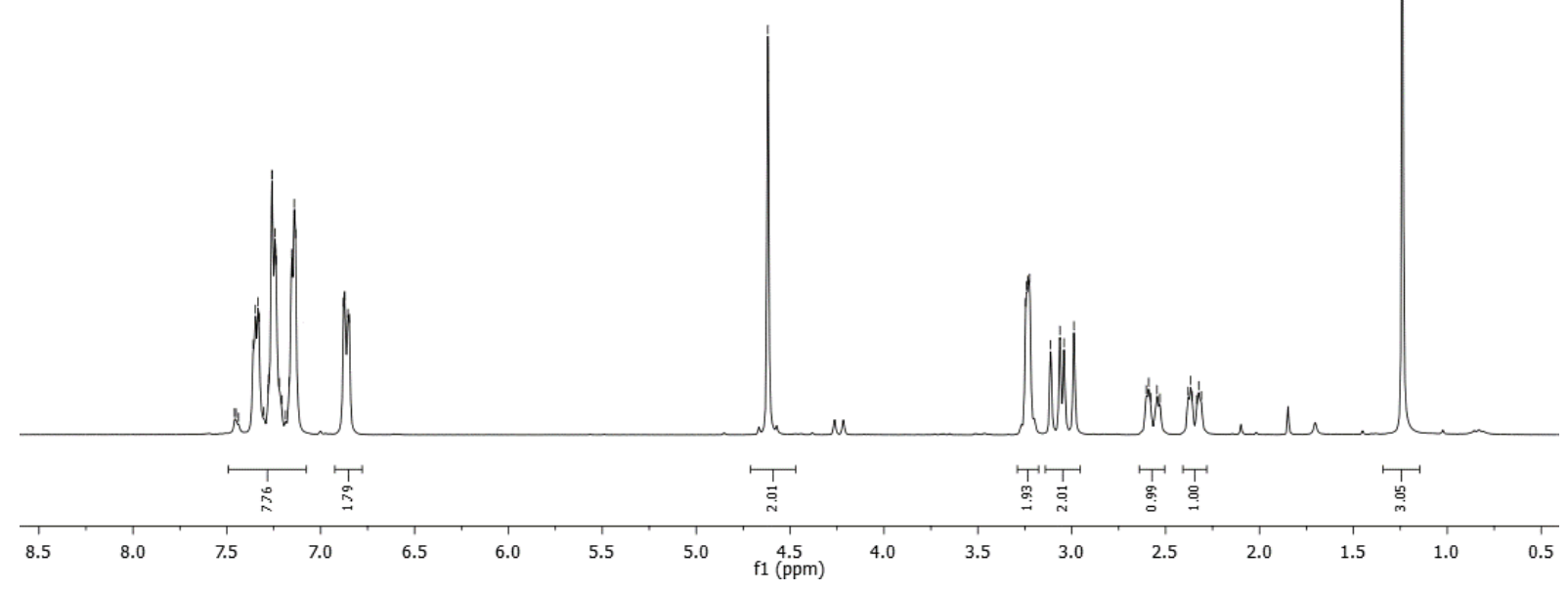

${ }^{13} \mathrm{C}$ NMR $\left(\mathrm{CDCl}_{3}, 101 \mathrm{MHz}\right)$

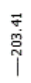

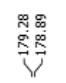

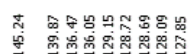

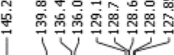

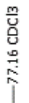

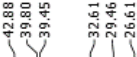

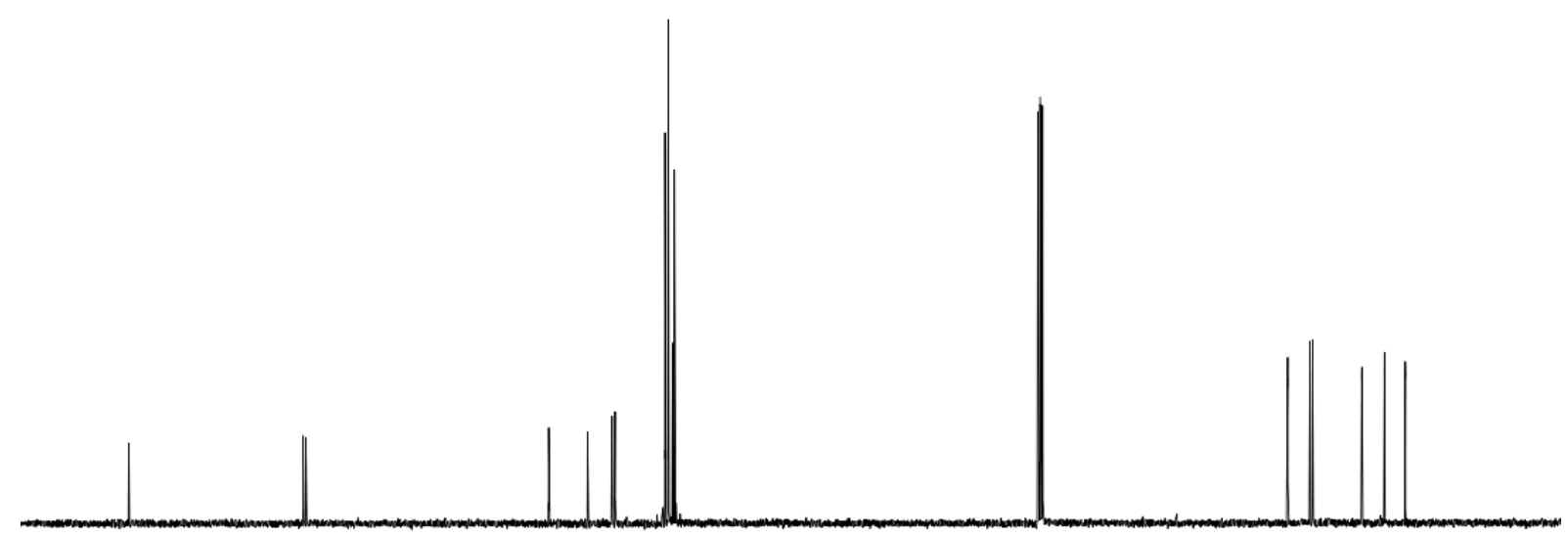

$\begin{array}{llllllllll}210 & 200 & 190 & 180 & 170 & 160 & 150 & 140 & 130 & 120\end{array}$ 


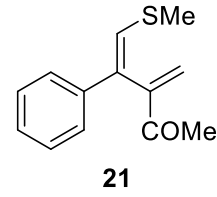

${ }^{1} \mathrm{H}$ NMR $\left(\mathrm{CDCl}_{3}, 400 \mathrm{MHz}\right)$

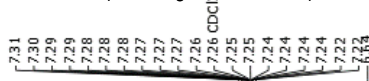

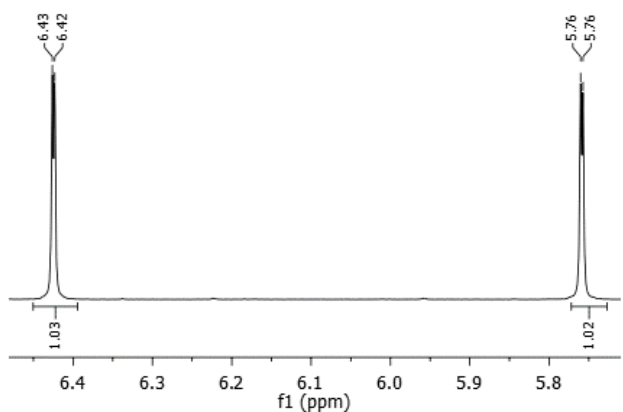

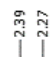

$\mid$

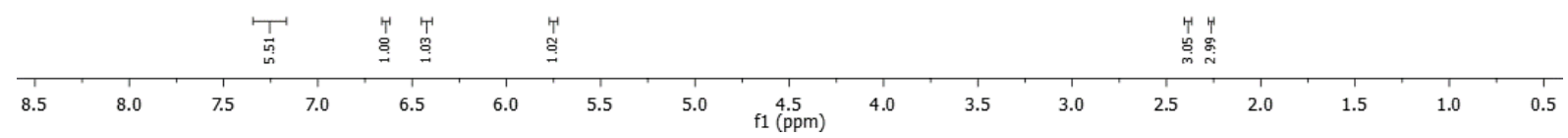

${ }^{13} \mathrm{C}$ NMR $\left(\mathrm{CDCl}_{3}, 101 \mathrm{MHz}\right)$
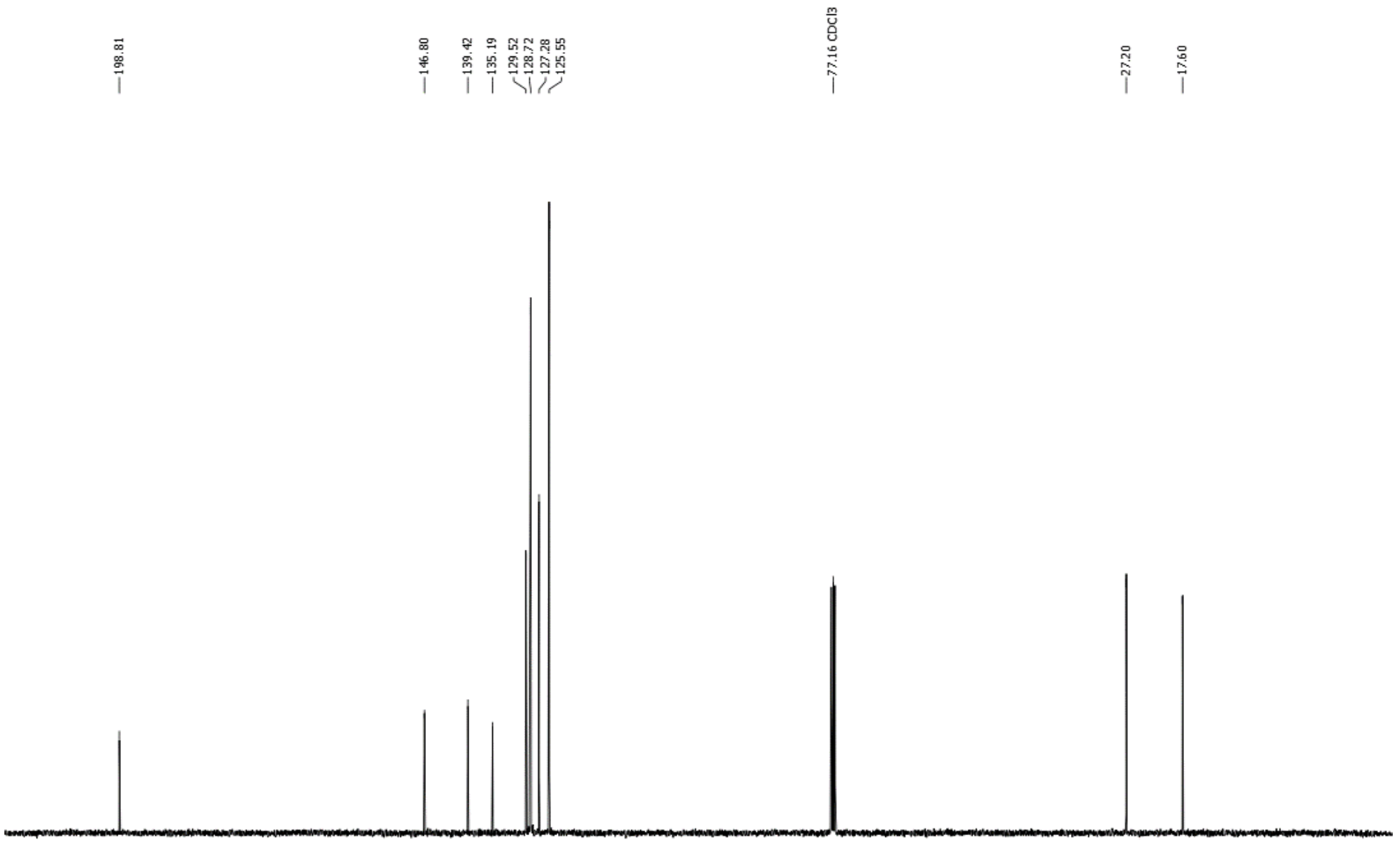

$\begin{array}{llllllllllllllllllllllllll}210 & 200 & 190 & 180 & 170 & 160 & 150 & 140 & 130 & 120 & 110 & 100 & 90 & 80 & 70 & 60 & 50 & 40 & 30 & 20 & 10 & 0 & -10\end{array}$ 


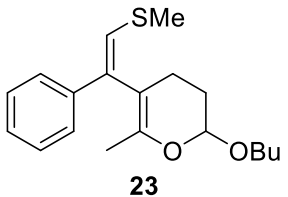

${ }^{1} \mathrm{H}$ NMR $\left(\mathrm{CDCl}_{3}, 400 \mathrm{MHz}\right)$

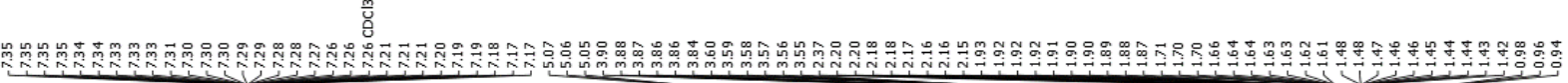

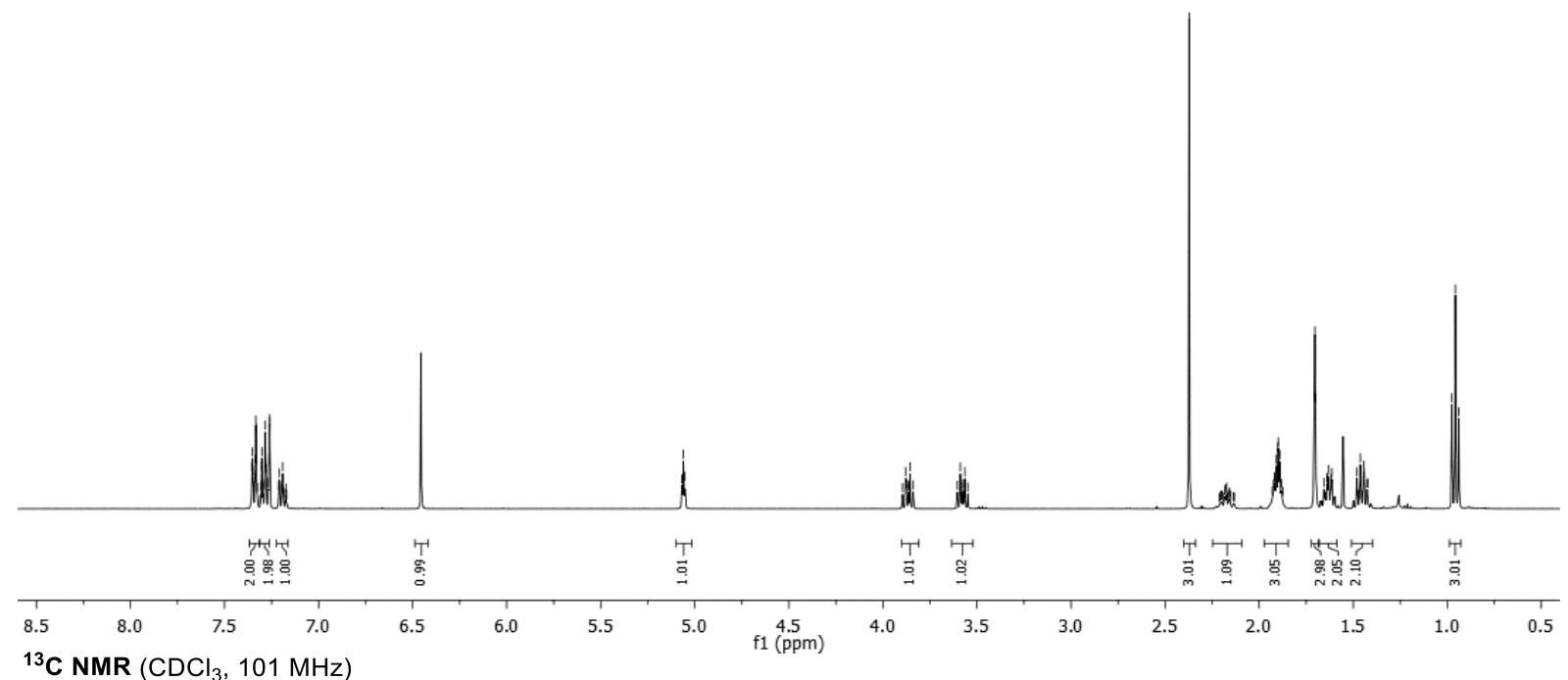

${ }^{13} \mathrm{C}$ NMR $\left(\mathrm{CDCl}_{3}, 101 \mathrm{MHz}\right)$
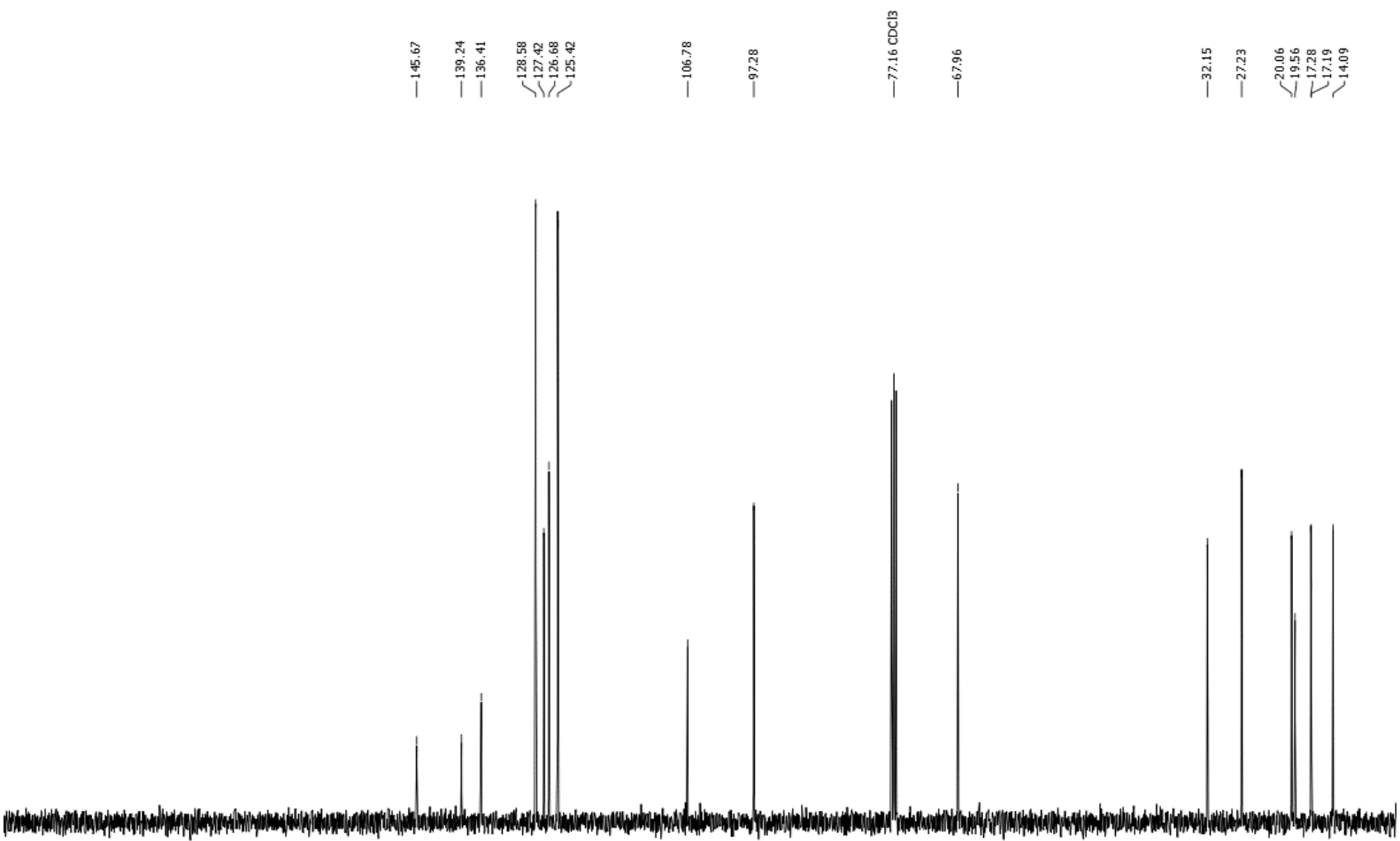

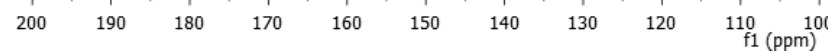




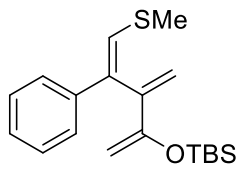

24

${ }^{1} \mathrm{H}$ NMR $\left(\mathrm{CDCl}_{3}, 400 \mathrm{MHz}\right)$

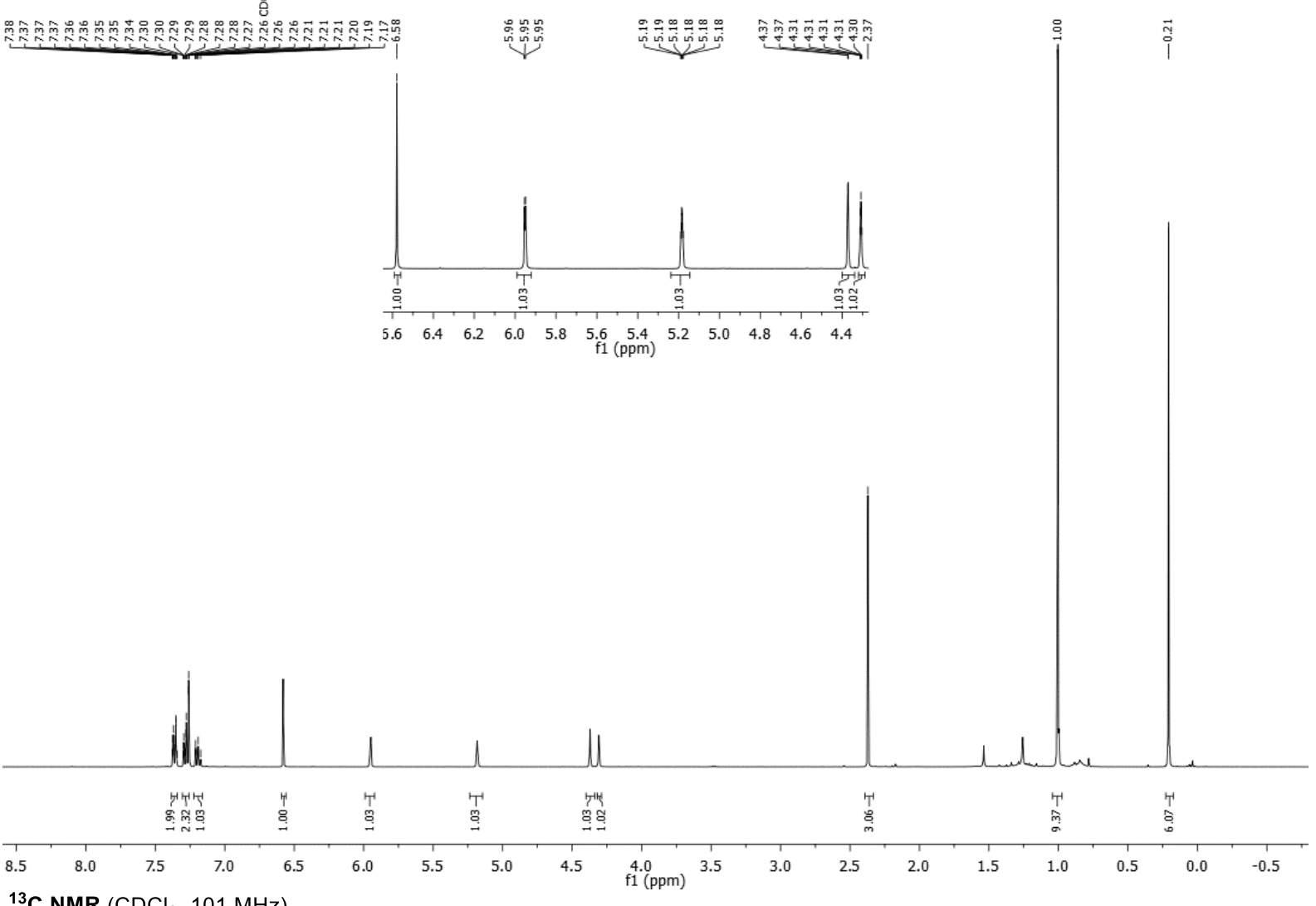

${ }^{13} \mathrm{C}$ NMR $\left(\mathrm{CDCl}_{3}, 101 \mathrm{MHz}\right)$

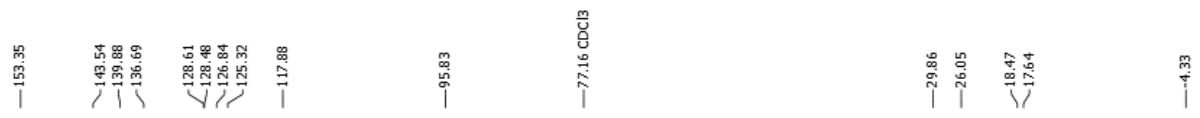

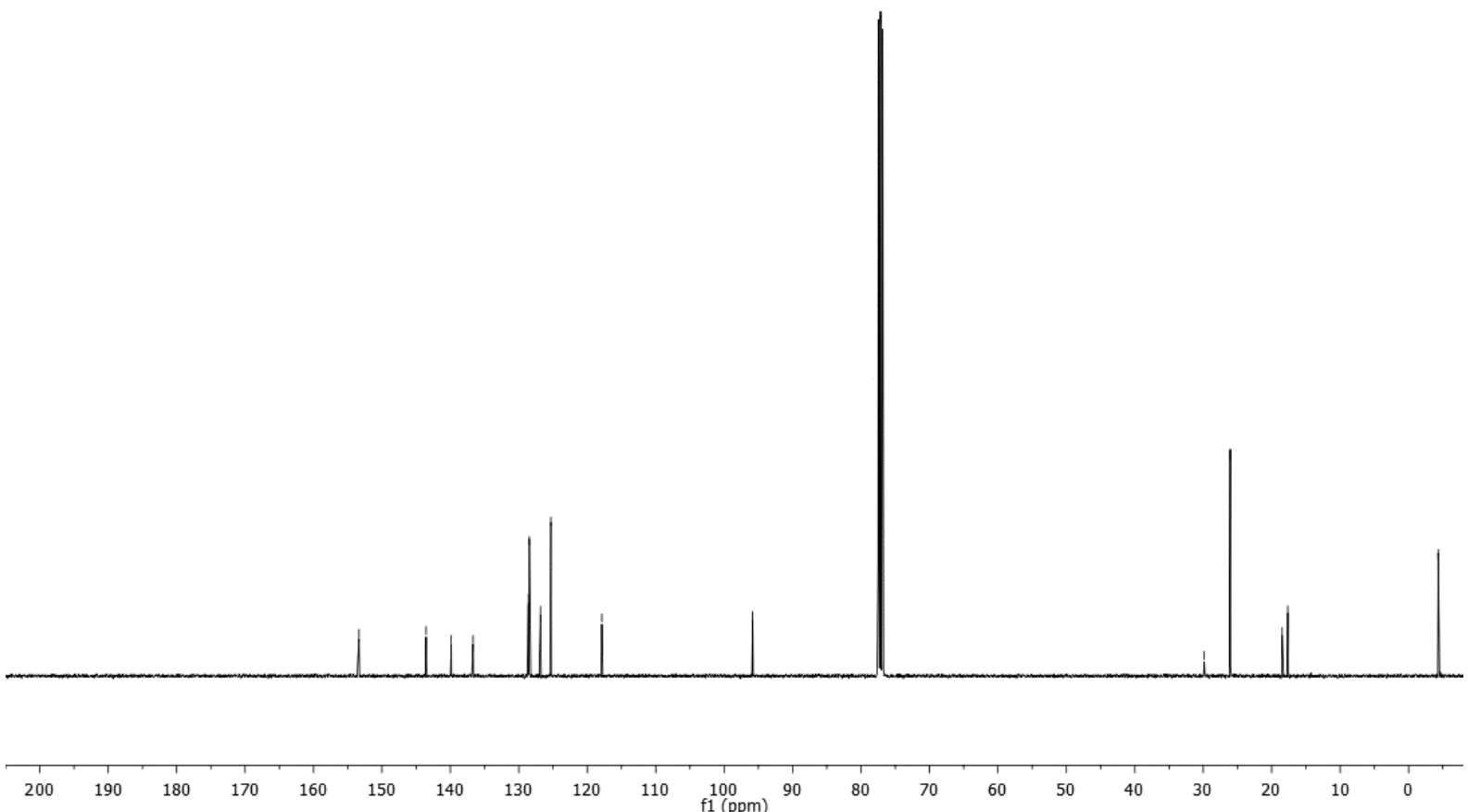




\section{$\mathrm{MeOOC}$ \\ $\mathrm{MeOOC}$}

26

${ }^{1} \mathrm{H} \mathrm{NMR}\left(\mathrm{CDCl}_{3}, 400 \mathrm{MHz}\right)$<smiles>[B][B]</smiles>
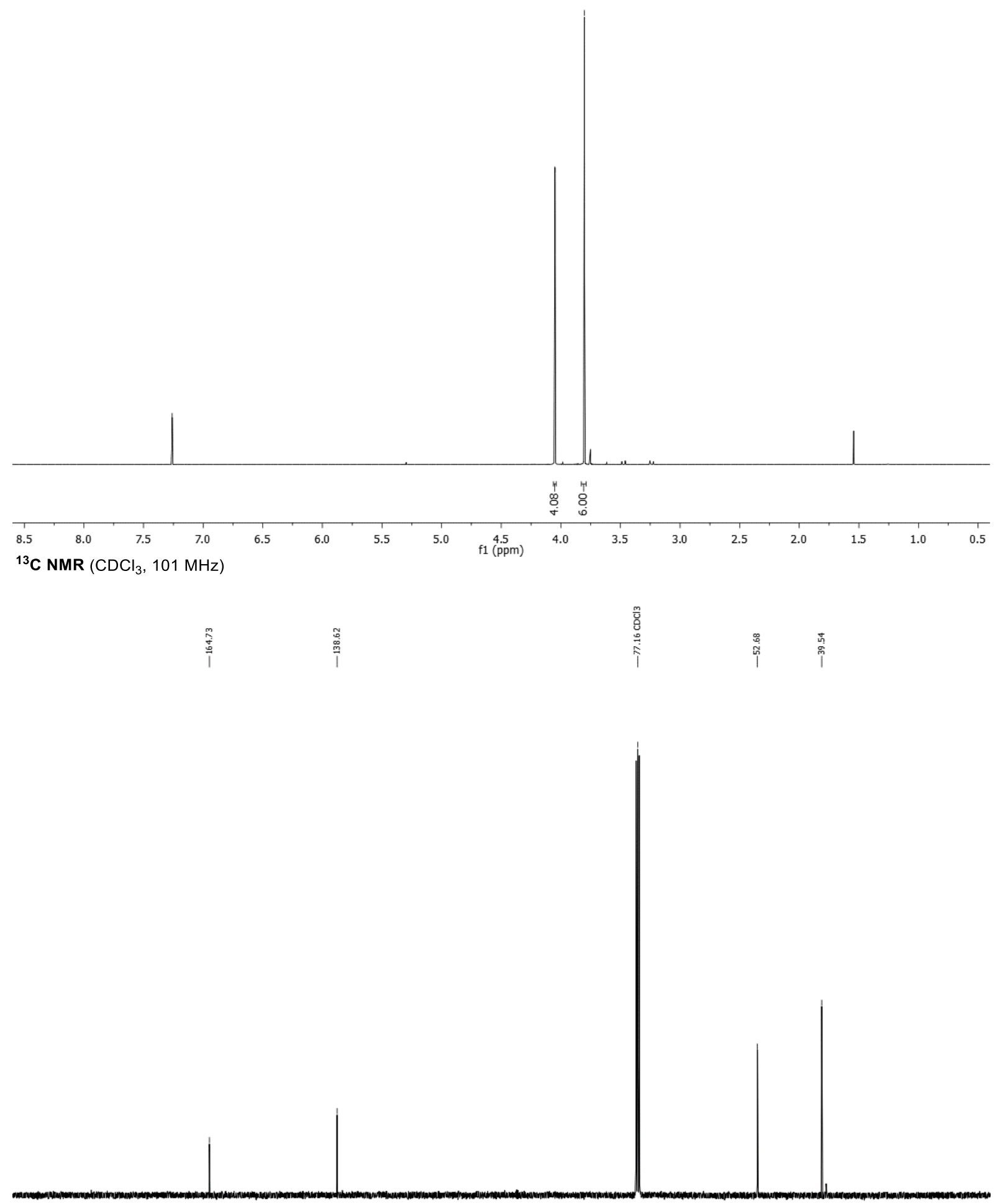

200

$\begin{array}{llllllllll}200 & 190 & 180 & 170 & 160 & 150 & 140 & 130 & 120 & 110 \\ (\mathrm{ppm}) & 100\end{array}$ 


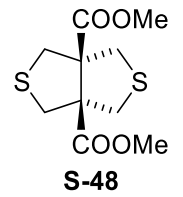

${ }^{1} \mathrm{H}$ NMR $\left(\mathrm{CDCl}_{3}, 400 \mathrm{MHz}\right)$

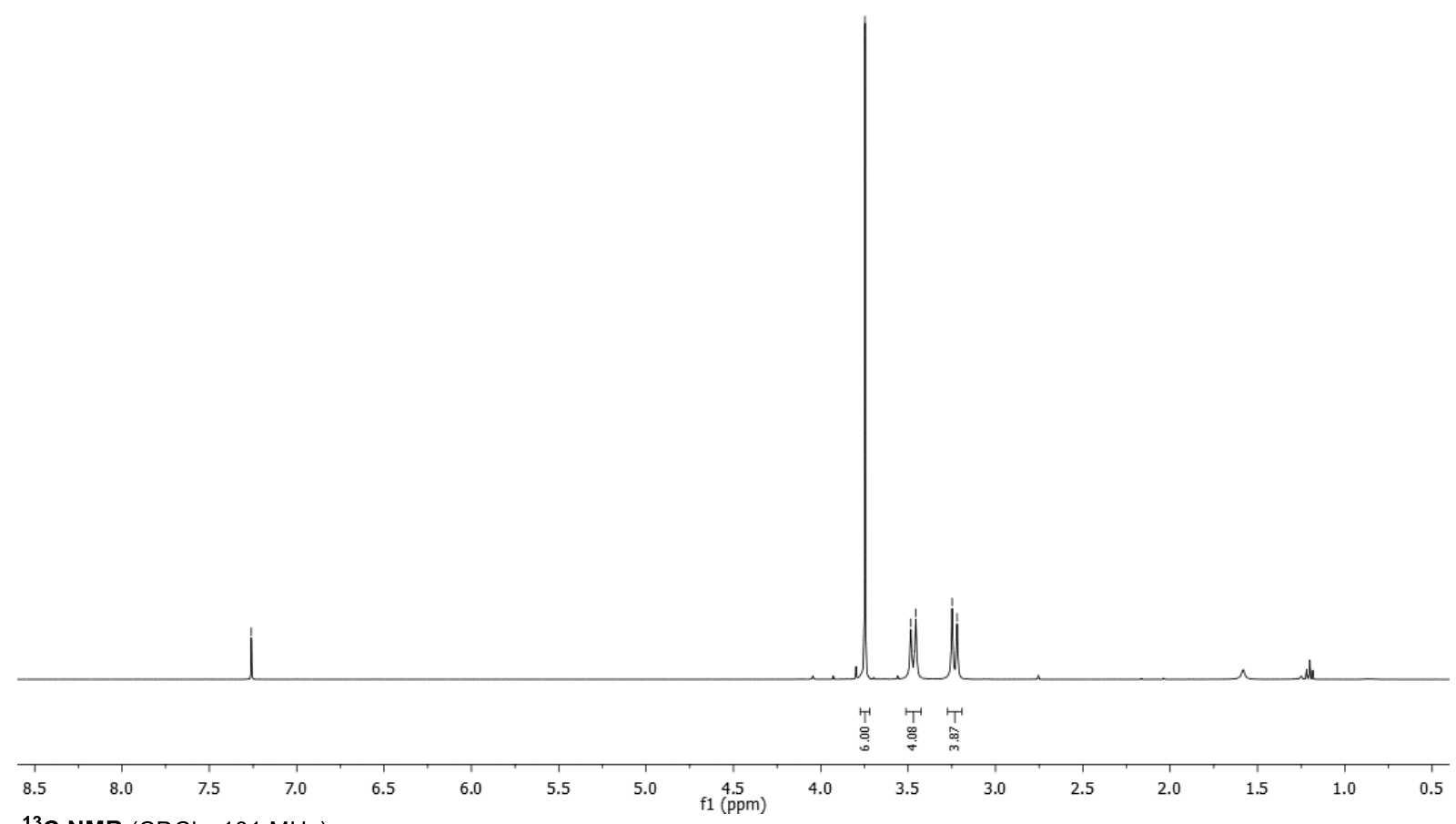

${ }^{13} \mathrm{C}$ NMR $\left(\mathrm{CDCl}_{3}, 101 \mathrm{MHz}\right)$

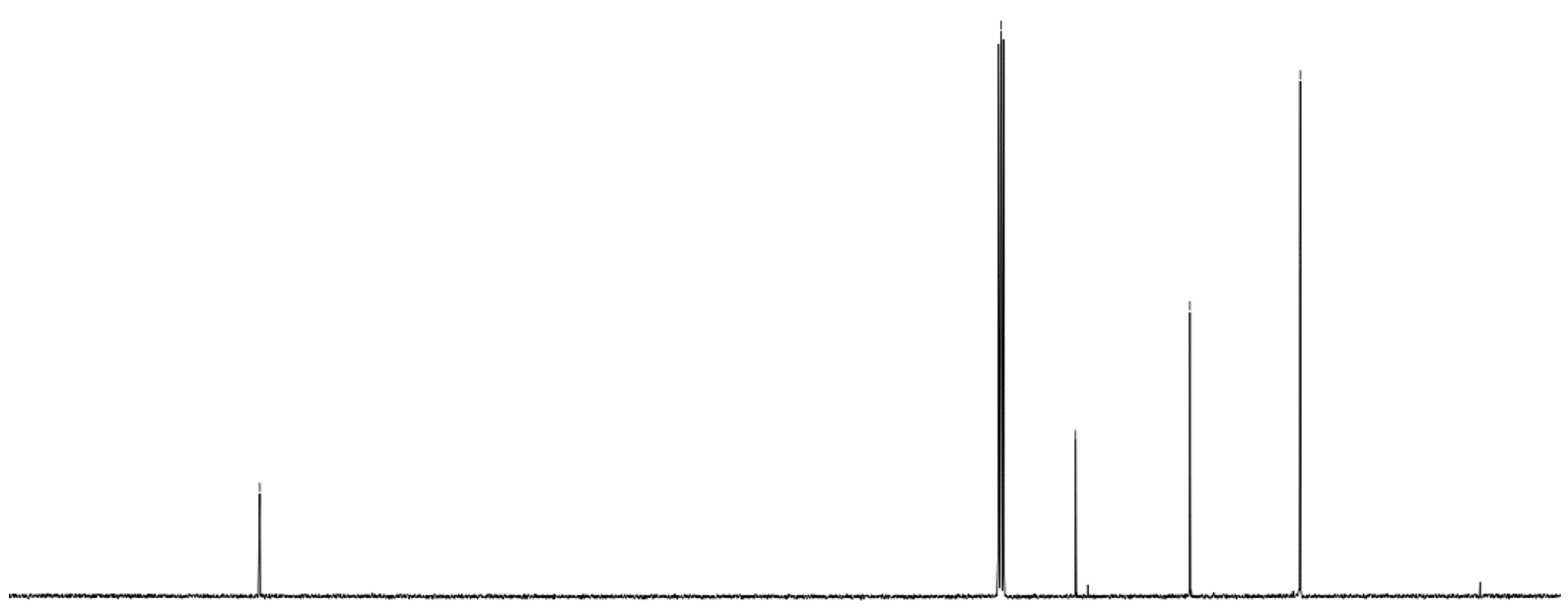

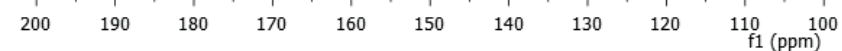




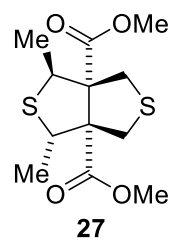

${ }^{1} \mathrm{H}$ NMR $\left(\mathrm{CDCl}_{3}, 400 \mathrm{MHz}\right)$
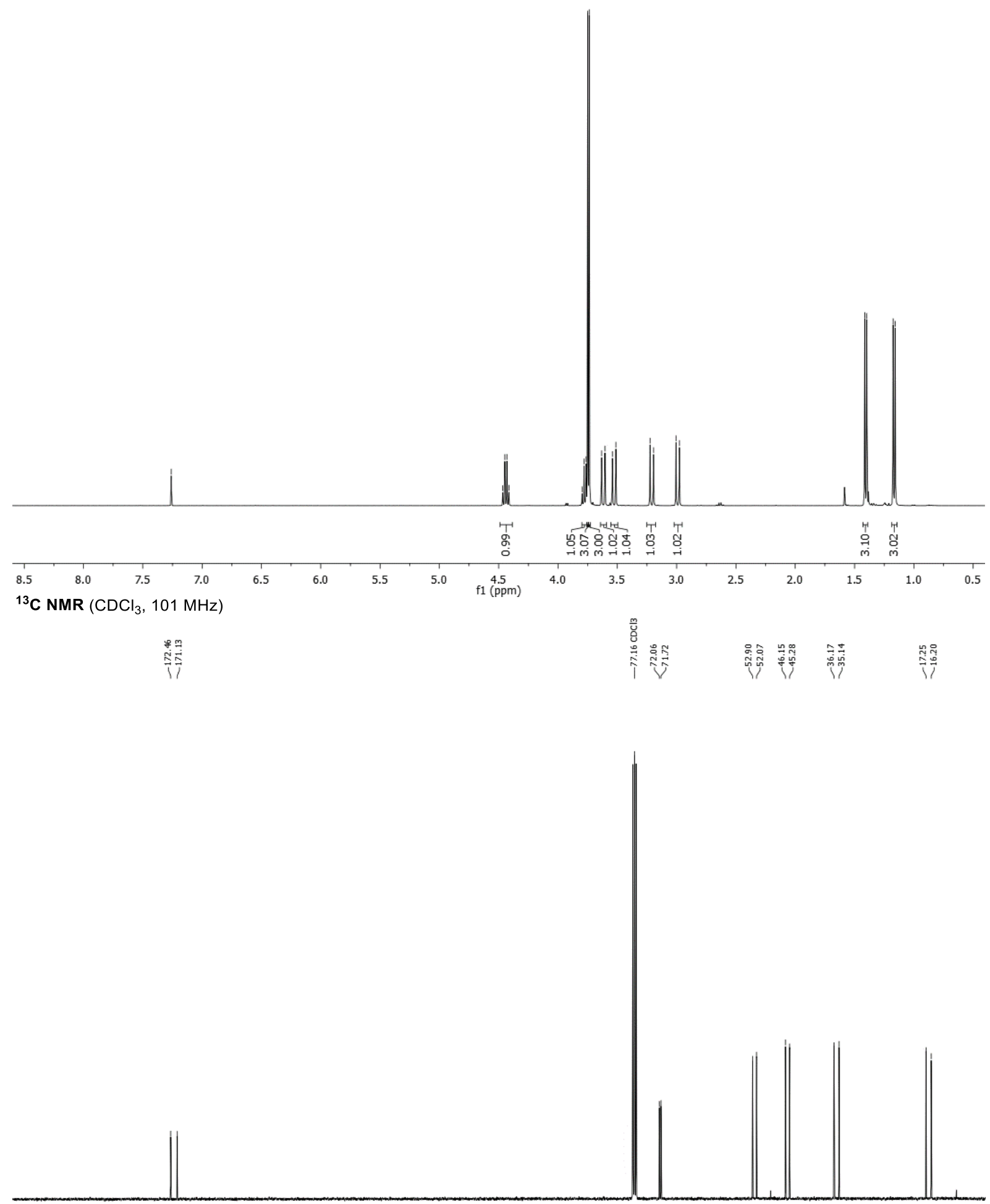


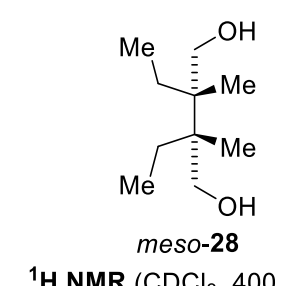

${ }^{1} \mathrm{H}$ NMR $\left(\mathrm{CDCl}_{3}, 400 \mathrm{MHz}\right)$
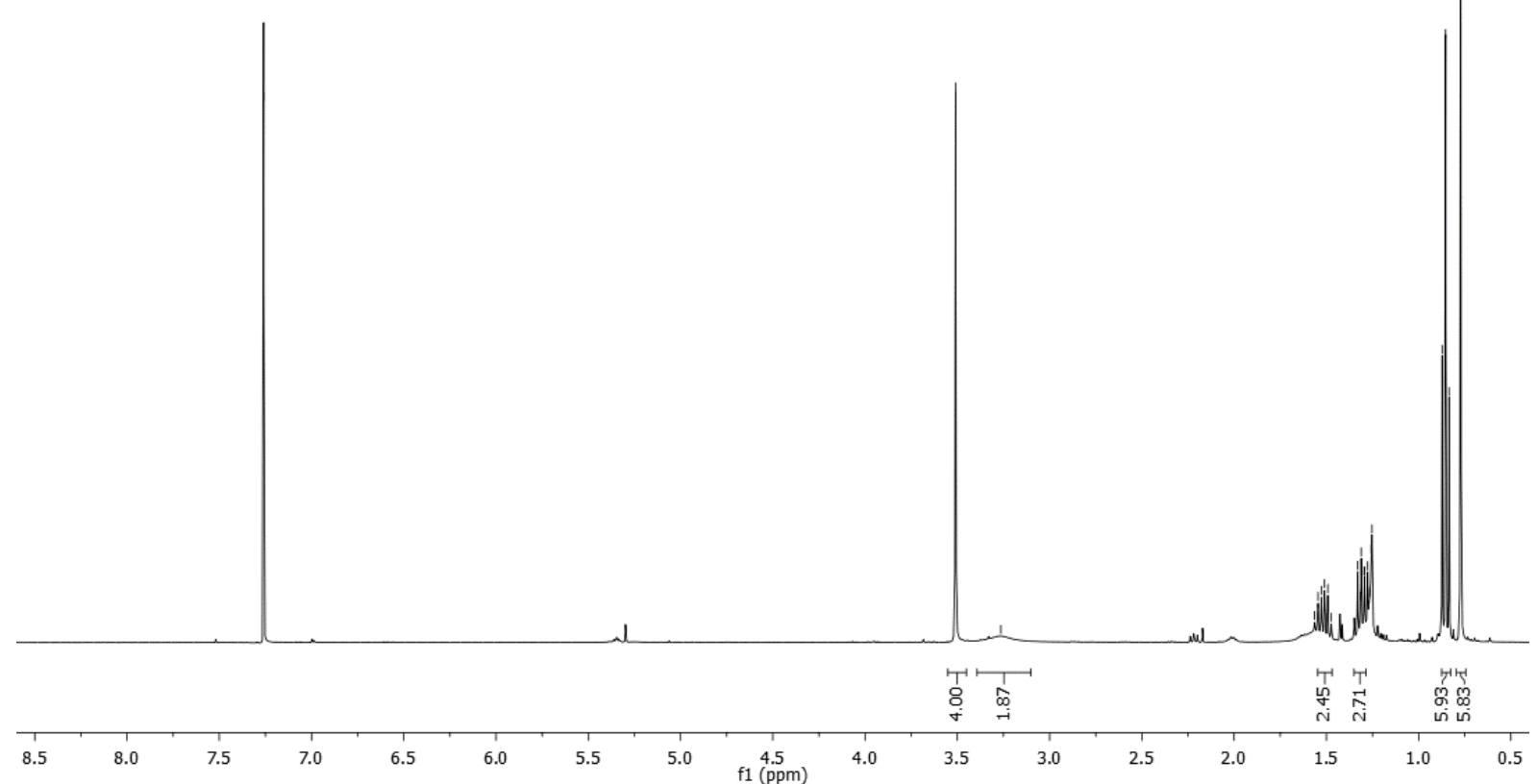

${ }^{13} \mathrm{C}$ NMR $\left(\mathrm{CDCl}_{3}, 101 \mathrm{MHz}\right)$
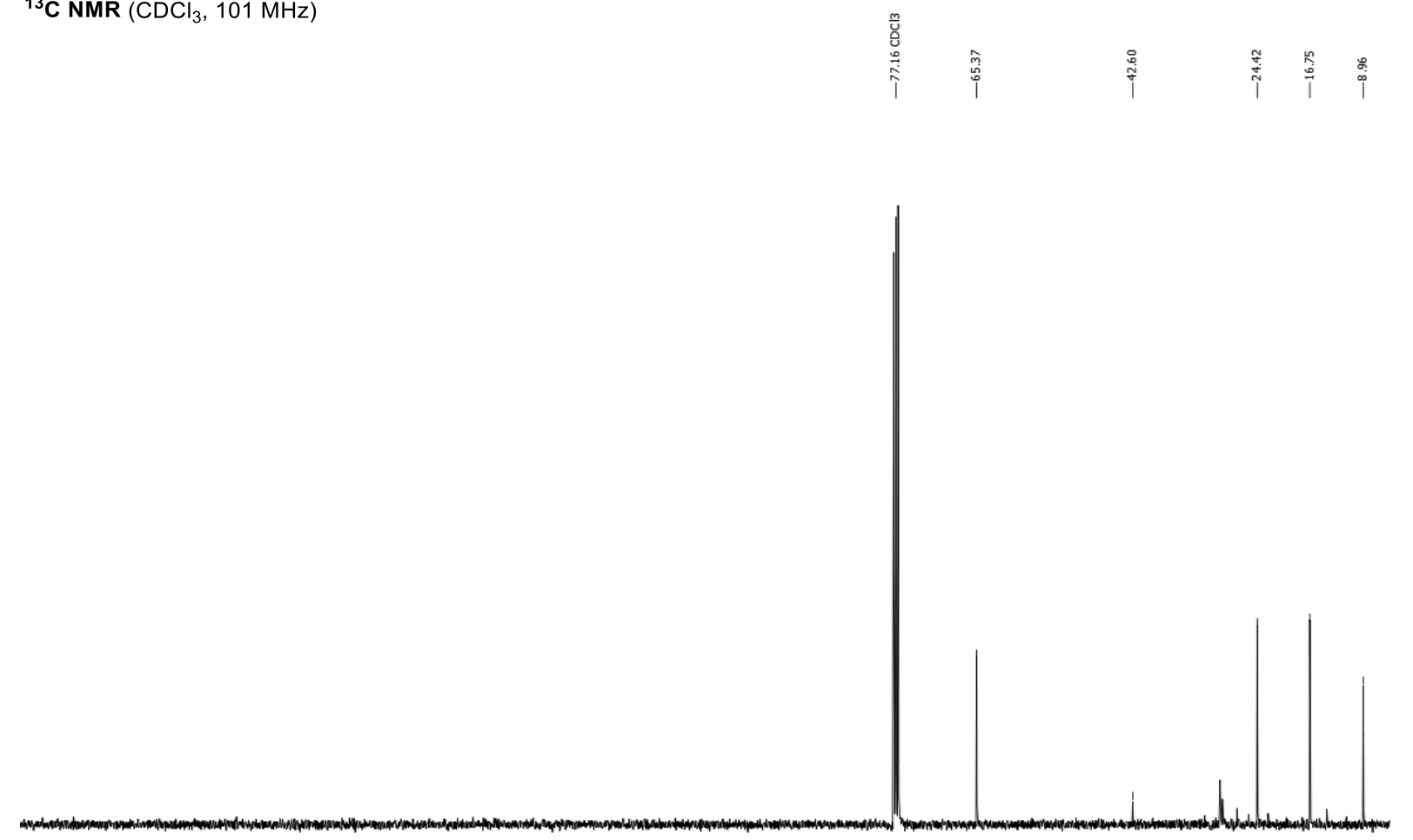

$\begin{array}{llllllllll}200 & 190 & 180 & 170 & 160 & 150 & 140 & 130 & 120 & \underset{\mathrm{f} 1(\mathrm{ppm})}{1100}\end{array}$ 


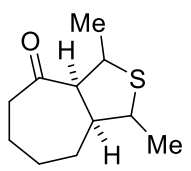

30

${ }^{1} \mathrm{H}$ NMR $\left(\mathrm{CDCl}_{3}, 400 \mathrm{MHz}\right)$

鱼

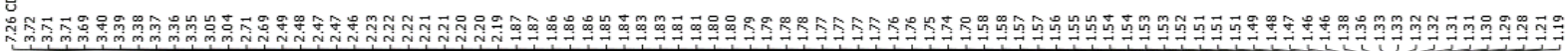

Alublum wh

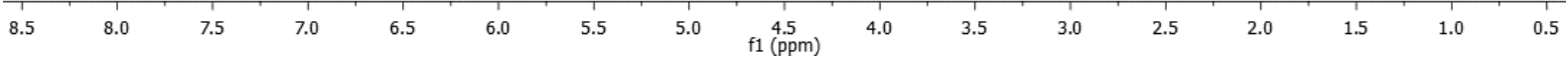

${ }^{13} \mathrm{C}$ NMR $\left(\mathrm{CDCl}_{3}, 101 \mathrm{MHz}\right)$

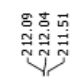

\section{熵}

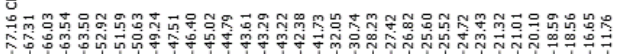

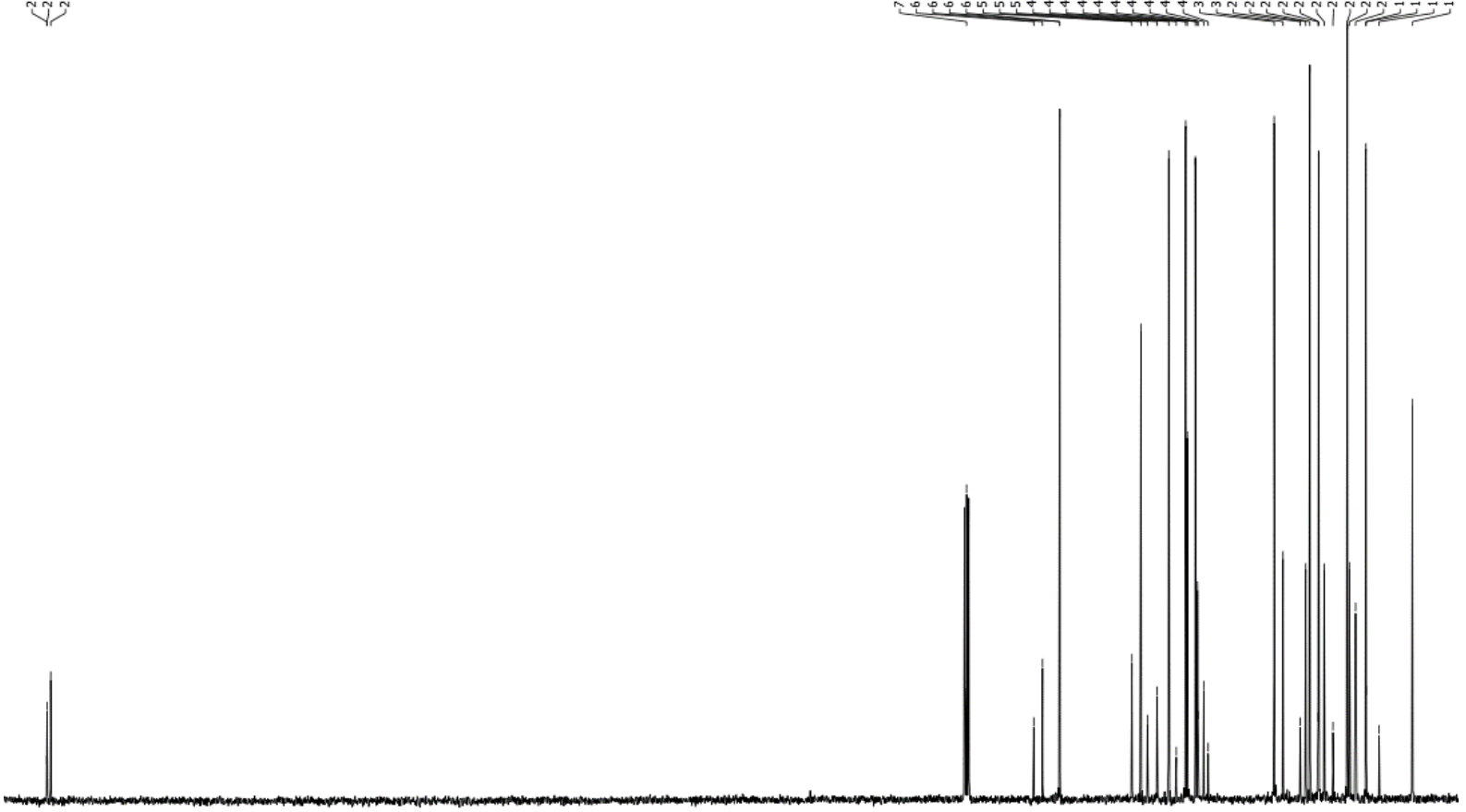

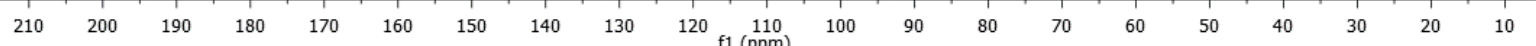




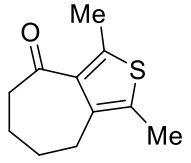

31

${ }^{1} \mathrm{H}$ NMR $\left(\mathrm{CDCl}_{3}, 600 \mathrm{MHz}\right)$

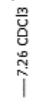

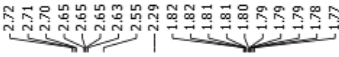

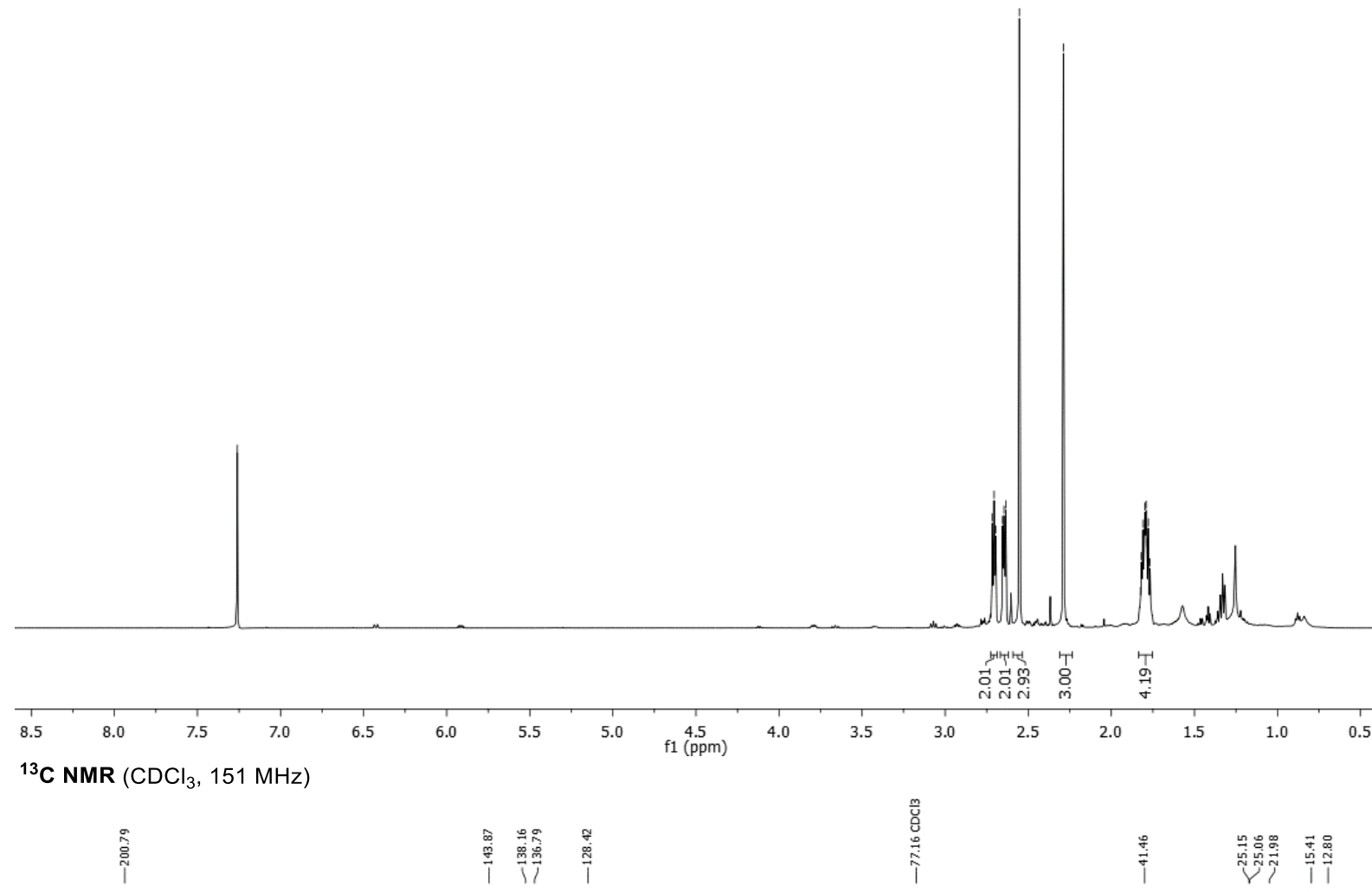

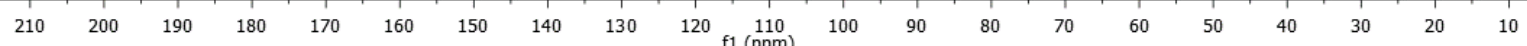


<smiles>COC1(OC)C=CC(=O)[C@H]2CSCC21</smiles>

$34 a$

${ }^{1} \mathrm{H}$ NMR $\left(\mathrm{CDCl}_{3}, 400 \mathrm{MHz}\right)$

章

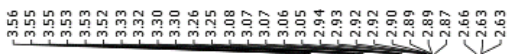
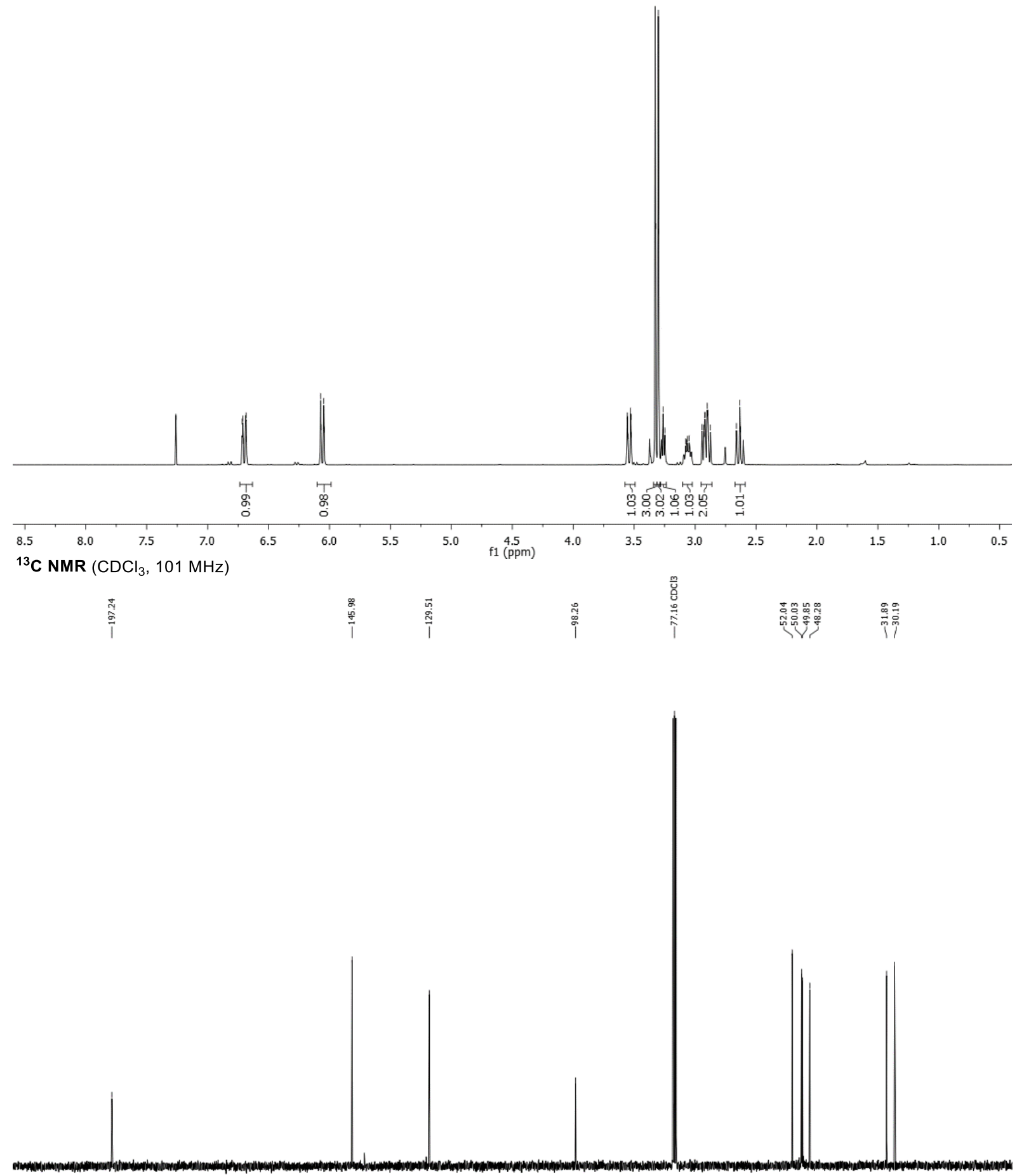

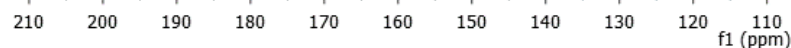




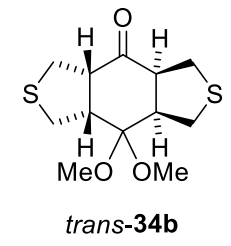

${ }^{1} \mathrm{H}$ NMR $\left(\mathrm{CDCl}_{3}, 400 \mathrm{MHz}\right)$

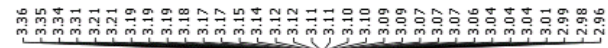

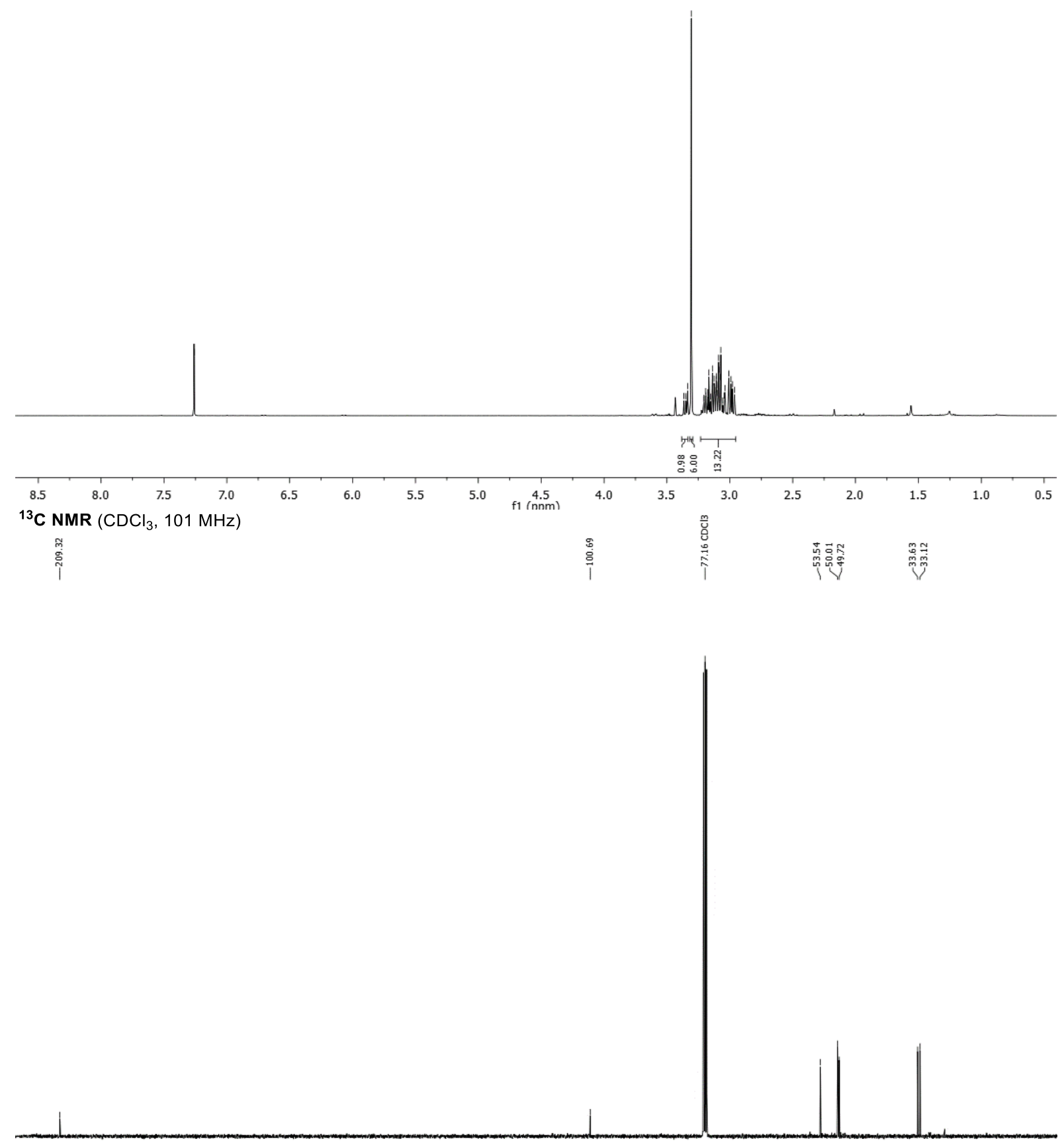

$\begin{array}{llllllllll}210 & 200 & 190 & 180 & 170 & 160 & 150 & 140 & 130 & 120 \\ \mathrm{f} 1(\mathrm{ppm})\end{array}$ 


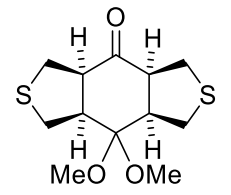

cis-34b

${ }^{1} \mathrm{H}$ NMR $\left(\mathrm{CDCl}_{3}, 400 \mathrm{MHz}\right)$

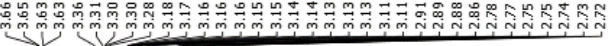
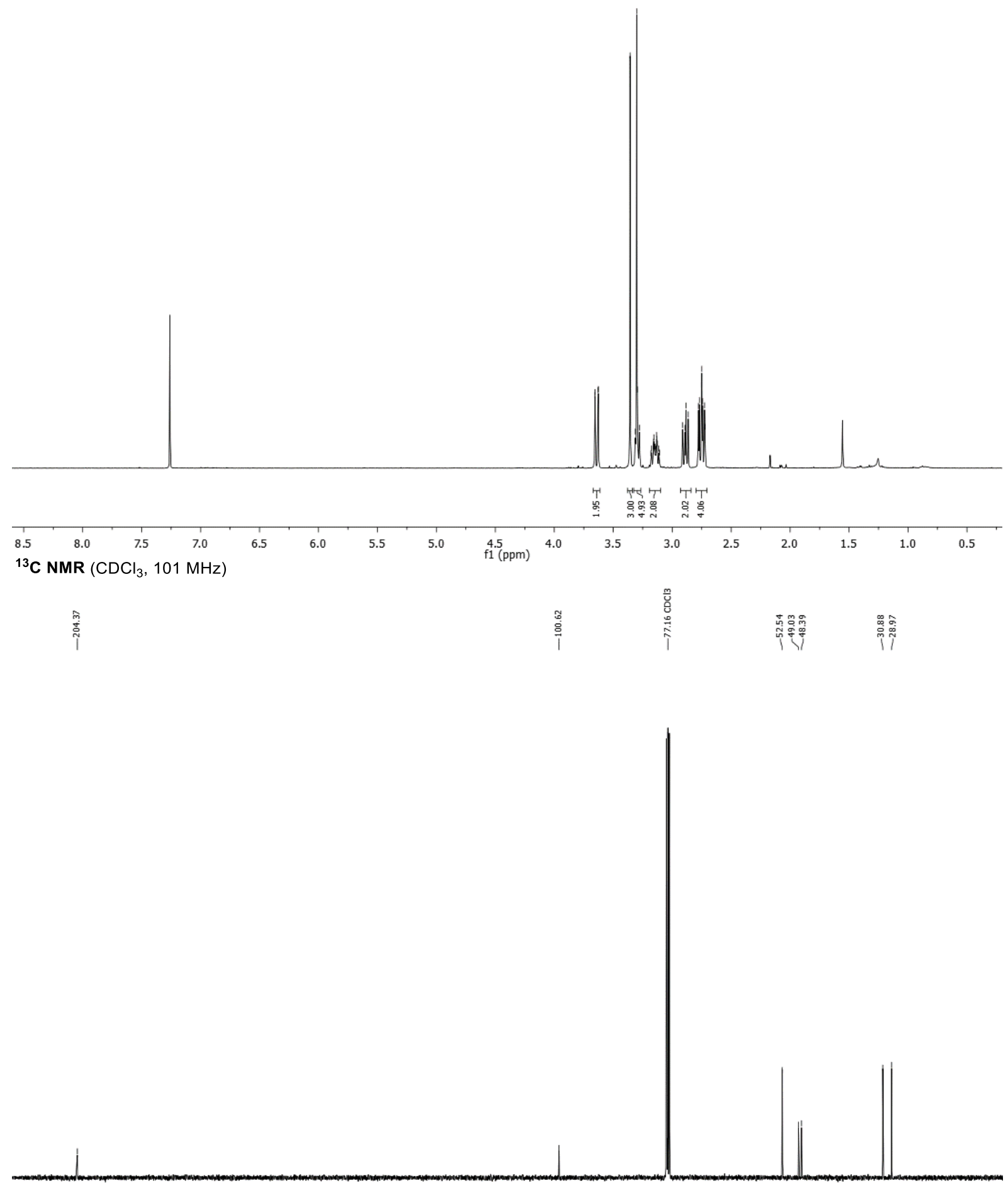

210 


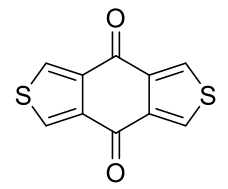

35

${ }^{1} \mathrm{H}$ NMR $\left(\mathrm{CD}_{2} \mathrm{Cl}_{2} / \mathrm{d}-\mathrm{TFA}, 300 \mathrm{MHz}\right)$

苟

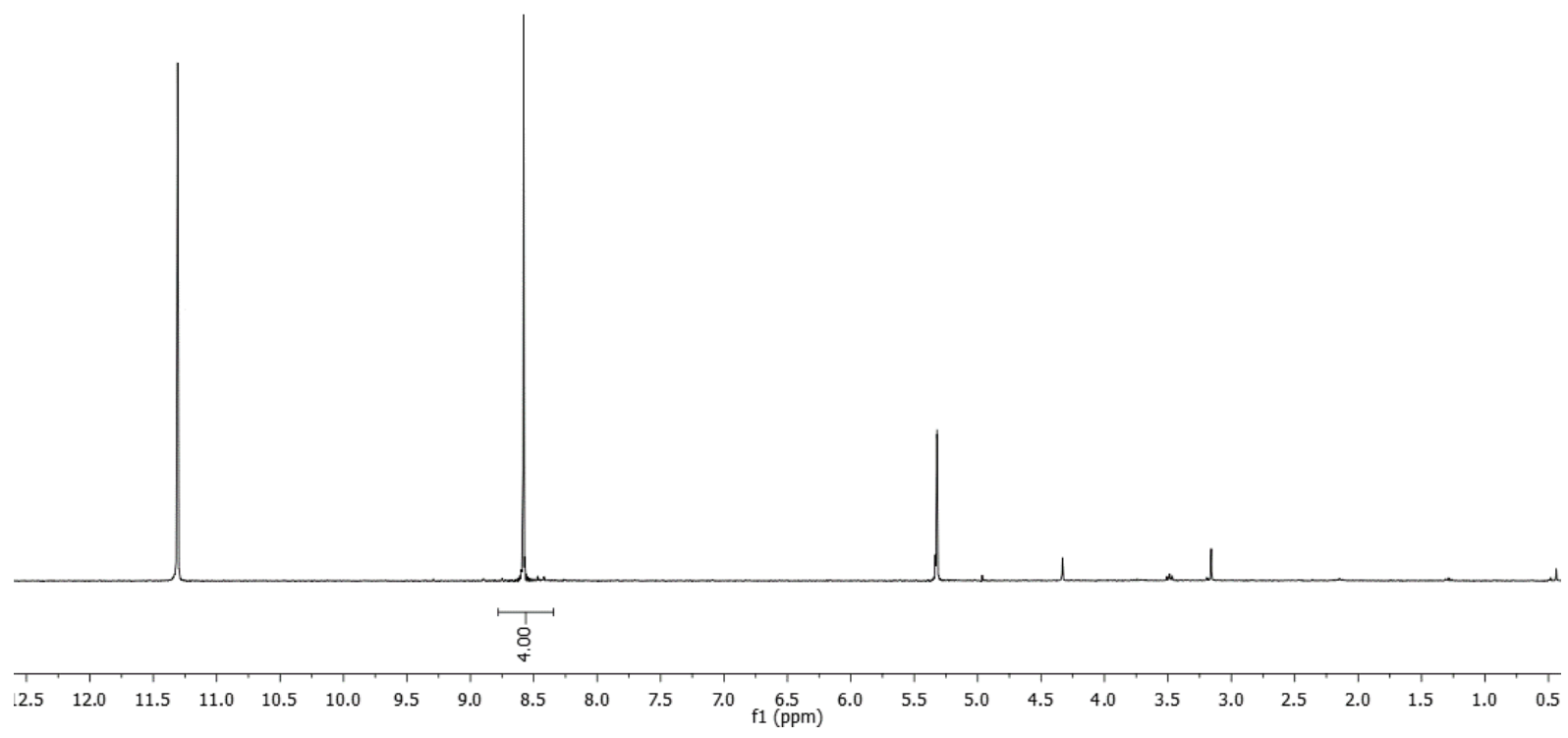

${ }^{13} \mathrm{C}$ NMR $\left(\mathrm{CD}_{2} \mathrm{Cl}_{2} / \mathrm{d}-\mathrm{TFA}, 75 \mathrm{MHz}\right)$
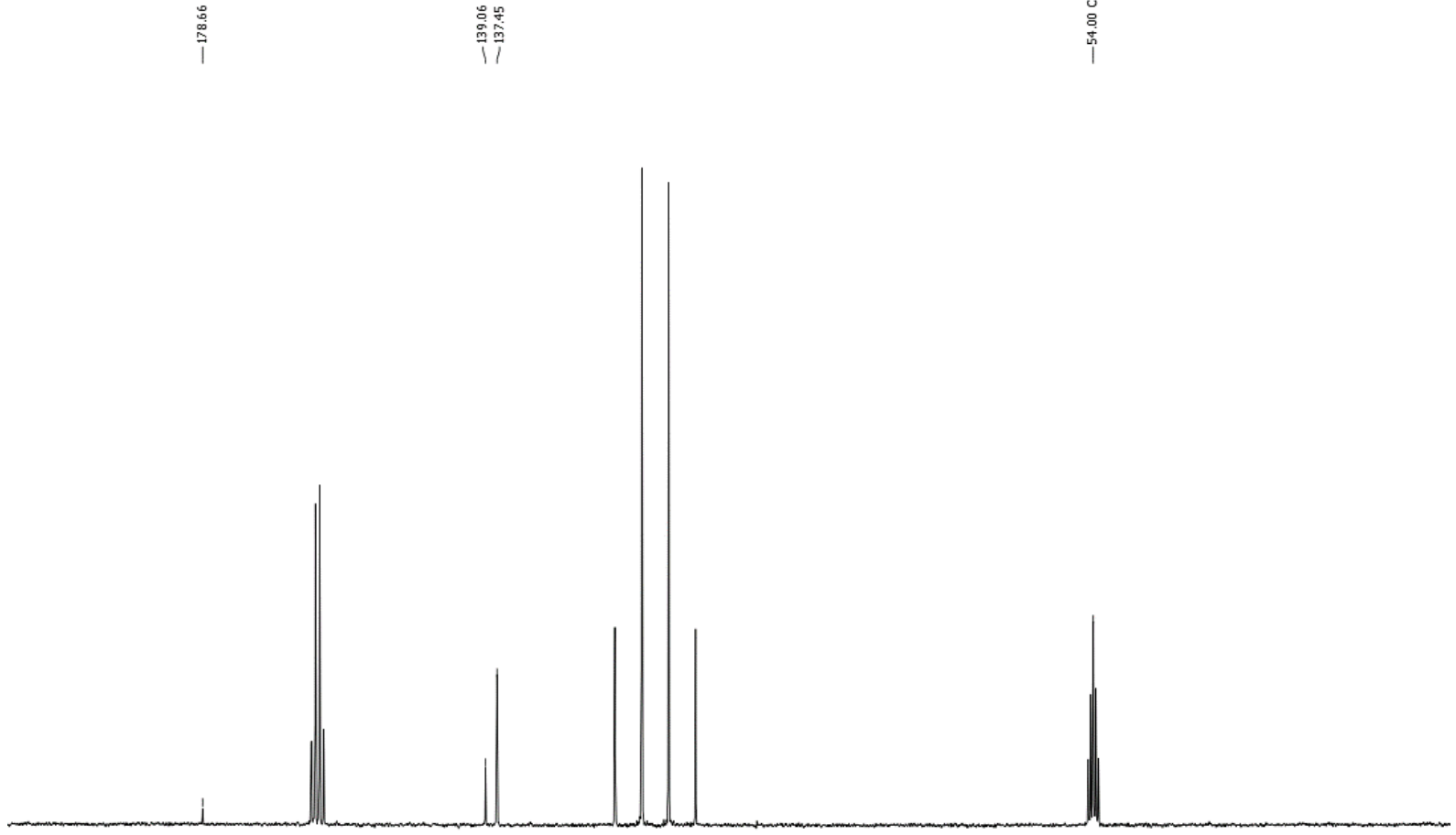

$\begin{array}{llllllllll}200 & 190 & 180 & 170 & 160 & 150 & 140 & 130 & 120 & 110 \\ \mathrm{f} 1(\mathrm{ppm}) & 100\end{array}$ 


\section{X-Ray Crystallographic Data}

X-ray diffraction analysis was carried out by Dr. Peter Mayer at LMU Munich. The data collections were performed either on an Oxford Diffraction Xcalibur diffractometer, on a Bruker D8Quest diffractometer or on a Bruker D8Venture at $100 \mathrm{~K}$ or at $173 \mathrm{~K}$ using MoKaradiation ( $\lambda=0.71073 \AA$, graphite monochromator). The CrysAlisPro software (version 1.171.33.41) was applied for the integration, scaling and multi-scan absorption correction of the data. The structures were solved by direct methods with SIR9730 and refined by leastsquares methods against F2 with SHELXL-97.31 All non-hydrogen atoms were refined anisotropically. The hydrogen atoms were placed in ideal geometry riding on their parent atoms. Further details are summarized in the tables at the different sections.

X-ray diffraction analysis was carried out by Prof. Dr. Klaus Wurst at LFU Innsbruck. The data collections were performed on a Bruker D8Quest using MoKa-radiation $(\lambda=0.71073 \AA$, Incoatec Microfocus). The Bruker Apex III software was applied for the integration, scaling and multi-scan absorption correction of the data. The structures were solved by direct methods with SHELXTL-XT-2014 and refined by least-squares methods against F2 with SHELXL2014/7. All non-hydrogen atoms were refined anisotropically. The hydrogen atoms were placed in ideal geometry riding on their parent atoms. Further details are summarized in the tables at the different sections.

\section{Tetrahydrothiophene (+)-8g}

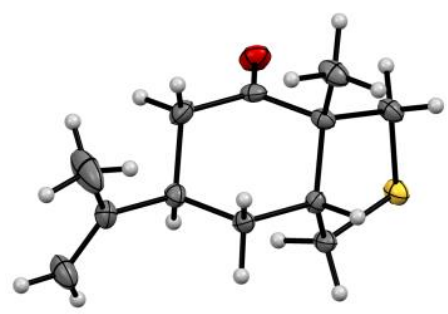

CCDC 1920377 contains the supplementary crystallographic data for (+)-8g.

Identification code

Empirical formula

Formula weight

Temperature

Wavelength

Crystal system

Space group

Unit cell dimensions vv273

$\mathrm{C}_{12} \mathrm{H}_{18} \mathrm{OS}$

210.32

$100(2) \mathrm{K}$

$0.71073 \AA$

Monoclinic

'P 1211 '

$\mathrm{a}=8.4949(2) \AA \quad \alpha=90^{\circ}$ 
Volume

Z

Density (calculated)

$\mu$

Absorption correction

Crystal size

Transmission factor range

Reflections collected

$R_{\text {int }}$

Mean $\sigma(I) / I$

$\theta$ range

Reflections observed

$x, y$ (weighting scheme)

Hydrogen refinement

Flack parameter

Reflections in refinement

Parameters

Restraints

$R\left(F_{\text {obs }}\right)$

$R_{w}\left(F^{2}\right)$

$S$

shift/error $\max$

Max. electron density

Min. electron density

$$
\begin{array}{ll}
b=6.0309(2) \AA & \beta=105.4090(10)^{\circ} \\
c=11.6385(5) \AA & Y=90^{\circ}
\end{array}
$$

574.83(4) $\AA^{3}$

2

$1.215 \mathrm{Mg} / \mathrm{m}^{3}$

$0.248 \mathrm{~mm}^{-1}$

Multi-Scan

$0.100 \times 0.040 \times 0.030 \mathrm{~mm}^{3}$

0.9130-0.9705

6622

0.0290

0.0314

3.448-26.350

2151

0.0226 and 0.2222

Constr.

$0.01(3)$

2272

129

1

0.0295

0.0730

1.085

0.001

0.267 e. $\AA^{-3}$

-0.197 e. $\AA^{-3}$

\section{Tetrahydrothiophene $8 \mathrm{~h}$}

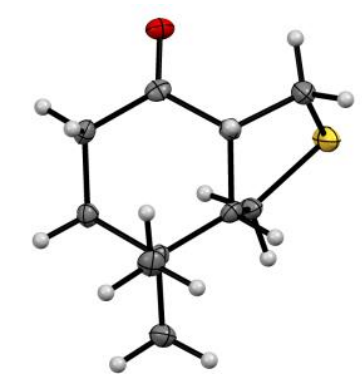

CCDC 1920379 contains the supplementary crystallographic data for $\mathbf{8 h}$. 
Empirical formula

Formula weight

Temperature

Wavelength

Crystal system

Space group

Unit cell dimensions

Volume

Z

Density (calculated)

$\mu$

Absorption correction

Crystal size

Transmission factor range

Reflections collected

$\mathrm{R}_{\text {int }}$

Mean $\sigma(I) / /$

$\theta$ range

Reflections observed

$\mathrm{x}, \mathrm{y}$ (weighting scheme)

Hydrogen refinement

Flack parameter

Reflections in refinement

Parameters

Restraints

$R\left(F_{\text {obs }}\right)$

$R_{w}\left(F^{2}\right)$

$S$

shift/error max $_{\text {m }}$

Max. electron density

Min. electron density
$\mathrm{C}_{10} \mathrm{H}_{16} \mathrm{OS}$

184.29

100(2) K

$0.71073 \AA$

Orthorhombic

'P 212121 '

$\begin{array}{ll}a=6.1557(3) \AA & \alpha=90^{\circ} \\ b=9.8405(5) \AA & \beta=90^{\circ} \\ c=16.0634(8) \AA & \gamma=90^{\circ}\end{array}$

973.04(8) $\AA^{3}$

4

$1.258 \mathrm{Mg} / \mathrm{m}^{3}$

$0.283 \mathrm{~mm}^{-1}$

Multi-Scan

$0.100 \times 0.070 \times 0.030 \mathrm{~mm}^{3}$

$0.8961-0.9705$

6902

0.0257

0.0264

3.274-26.355

1874

0.0188 and 0.4009

Constr.

0.5

1985

111

0

0.0283

0.0645

1.093

0.001

0.236 e. $\AA^{-3}$

-0.184 e. $\AA^{-3}$ 


\section{Tetrahydrothiophene $8 \mathbf{i}$}

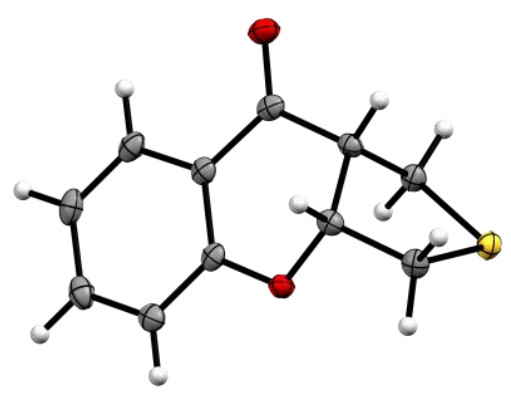

CCDC 1920378 contains the supplementary crystallographic data for $\mathbf{8 i}$.

\begin{tabular}{|c|c|c|}
\hline Identification code & \multicolumn{2}{|l|}{ vv448 } \\
\hline Empirical formula & \multicolumn{2}{|l|}{$\mathrm{C}_{11} \mathrm{H}_{10} \mathrm{O}_{2} \mathrm{~S}$} \\
\hline Formula weight & \multicolumn{2}{|l|}{206.25} \\
\hline Temperature & \multicolumn{2}{|l|}{$100(2) \mathrm{K}$} \\
\hline Wavelength & \multicolumn{2}{|l|}{$0.71073 \AA$} \\
\hline Crystal system & \multicolumn{2}{|l|}{ Orthorhombic } \\
\hline Space group & \multicolumn{2}{|l|}{ 'P b c a' } \\
\hline \multirow[t]{3}{*}{ Unit cell dimensions } & $a=6.8135(2) \AA$ & $\alpha=90^{\circ}$ \\
\hline & $b=12.9983(4)$ & $\beta=90^{\circ}$ \\
\hline & $c=20.5368(7) \AA$ & $Y=90^{\circ}$ \\
\hline Volume & \multicolumn{2}{|l|}{$1818.82(10) \AA^{3}$} \\
\hline Z & \multicolumn{2}{|l|}{8} \\
\hline Density (calculated) & \multicolumn{2}{|l|}{$1.506 \mathrm{Mg} / \mathrm{m}^{3}$} \\
\hline$\mu$ & \multicolumn{2}{|l|}{$0.321 \mathrm{~mm}^{-1}$} \\
\hline Absorption correction & \multicolumn{2}{|c|}{ Multi-Scan } \\
\hline Crystal size & \multicolumn{2}{|c|}{$0.100 \times 0.070 \times 0.010 \mathrm{~mm}^{3}$} \\
\hline Transmission factor range & \multicolumn{2}{|l|}{$0.8999-0.9705$} \\
\hline Reflections collected & \multicolumn{2}{|l|}{29927} \\
\hline $\mathrm{R}_{\text {int }}$ & \multicolumn{2}{|l|}{0.0522} \\
\hline Mean $\sigma(I) / I$ & \multicolumn{2}{|l|}{0.0187} \\
\hline$\theta$ range & \multicolumn{2}{|l|}{$3.519-26.366$} \\
\hline Reflections observed & \multicolumn{2}{|l|}{1690} \\
\hline x, y (weighting scheme) & \multicolumn{2}{|l|}{0.0285 and 1.9402} \\
\hline Hydrogen refinement & \multicolumn{2}{|l|}{ Constr. } \\
\hline Reflections in refinement & \multicolumn{2}{|l|}{1869} \\
\hline Parameters & \multicolumn{2}{|l|}{127} \\
\hline Restraints & \multicolumn{2}{|l|}{0} \\
\hline$R\left(F_{\text {obs }}\right)$ & \multicolumn{2}{|l|}{0.0347} \\
\hline
\end{tabular}



$R_{w}\left(F^{2}\right)$
0.0817
$S$
1.090
shift/error $\max$
0.001
Max. electron density
0.347 e. $\AA^{-3}$
Min. electron density
-0.233 e. $\AA^{-3}$

\section{Tetrahydrothiophene $8 \mathrm{n}$}

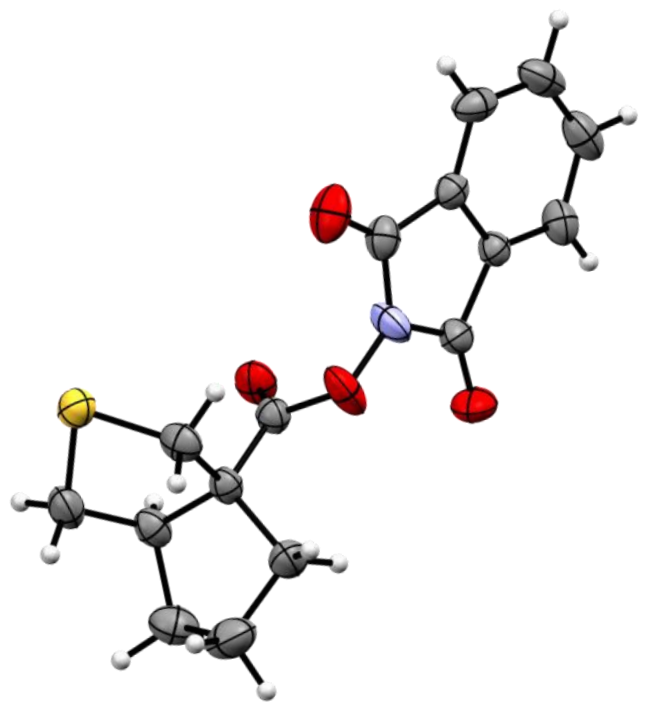

CCDC 1921467 contains the supplementary crystallographic data for $\mathbf{8 n}$.

Identification code
Empirical formula
Formula weight
Temperature
Wavelength
Crystal system
Space group
Unit cell dimensions

Volume

Z

Density (calculated)

Absorption coefficient

$F(000)$

Crystal size

Theta range for data collection mar18n12

$\mathrm{C}_{16} \mathrm{H}_{15} \mathrm{NO}_{4} \mathrm{~S}$

317.35

213(2) K

$0.71073 \AA$

Triclinic

P-1 (no. 2)

$\mathrm{a}=7.6475(4) \AA \quad \alpha=105.357(1)^{\circ}$

$\mathrm{b}=8.1332(4) \AA \quad \beta=102.201(2)^{\circ}$

$c=13.0597(6) \AA \quad Y=97.465(1)^{\circ}$

$750.56(6) \AA^{3}$

2

$1.404 \mathrm{Mg} / \mathrm{m}^{3}$

$0.233 \mathrm{~mm}^{-1}$

332

$0.180 \times 0.110 \times 0.070 \mathrm{~mm}^{3}$

2.649 to $24.998^{\circ}$ 
Index ranges

Reflections collected

Independent reflections

Completeness to theta $=24.998^{\circ}$

Absorption correction

Max. and min. transmission

Refinement method

Data / restraints / parameters

Goodness-of-fit on $\mathrm{F}^{2}$

Final $R$ indices [l>2sigma(I)]

$R$ indices (all data)

Extinction coefficient

Largest diff. peak and hole $-9<=\mathrm{h}<=9,-9<=\mathrm{k}<=9,-15<=\mathrm{l}<=15$

19592

$2644[R$ (int) $=0.0251]$

$100.0 \%$

Semi-empirical from equivalents

0.971 and 0.913

Full-matrix least-squares on $\mathrm{F}^{2}$

2644 / 0 / 219

1.041

$\mathrm{R} 1=0.0323, \mathrm{wR} 2=0.0821$

$\mathrm{R} 1=0.0368, \mathrm{wR} 2=0.0846$

$0.041(3)$

0.249 and -0.155 e. $\AA^{-3}$

\section{Tetrahydrothiophene $8 p$}

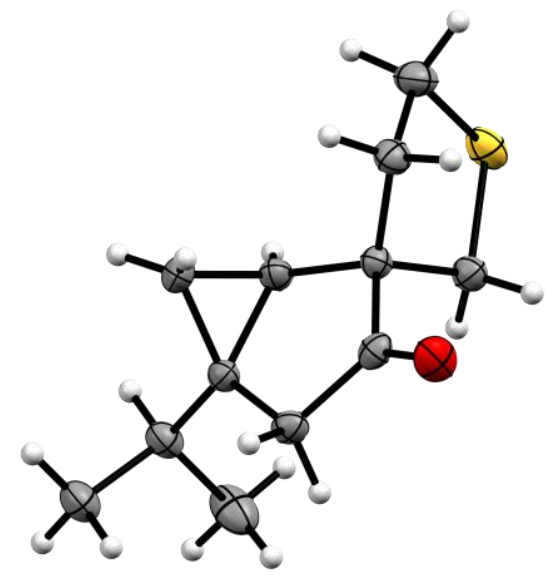

CCDC 1921468 contains the supplementary crystallographic data for $8 p$.

Identification code

Empirical formula

Formula weight

Temperature

Wavelength

Crystal system

Space group

Unit cell dimensions mar19-8

$\mathrm{C}_{12} \mathrm{H}_{18} \mathrm{OS}$

210.32

183(2) K

$0.71073 \AA$

Orthorhombic

P212121 (no. 19)

$$
\begin{array}{ll}
a=5.8430(4) \AA & \alpha=90^{\circ} \\
b=10.9977(8) \AA & \beta=90^{\circ} \\
c=17.5865(12) \AA & Y=90^{\circ}
\end{array}
$$


Volume

Z

Density (calculated)

Absorption coefficient

$\mathrm{F}(000)$

Crystal size

Theta range for data collection

Index ranges

Reflections collected

Independent reflections

Completeness to theta $=25.242^{\circ}$

Absorption correction

Max. and min. transmission

Refinement method

Data / restraints / parameters

Goodness-of-fit on $\mathrm{F}^{2}$

Final $R$ indices [l>2sigma(I)]

$\mathrm{R}$ indices (all data)

Absolute structure parameter

Extinction coefficient

Largest diff. peak and hole
$1130.10(14) \AA^{3}$

4

$1.236 \mathrm{Mg} / \mathrm{m}^{3}$

$0.253 \mathrm{~mm}^{-1}$

456

$0.200 \times 0.180 \times 0.015 \mathrm{~mm}^{3}$

2.316 to $25.398^{\circ}$

$-7<=\mathrm{h}<=7,-12<=\mathrm{k}<=13,-21<=\mathrm{k}<=21$

26482

2061 [R(int) $=0.0415]$

$99.9 \%$

Semi-empirical from equivalents

0.977 and 0.931

Full-matrix least-squares on $\mathrm{F}^{2}$

$2061 / 3 / 140$

1.066

$R 1=0.0225, w R 2=0.0569$

$\mathrm{R} 1=0.0251, \mathrm{wR} 2=0.0580$

$-0.025(16)$

$0.020(3)$

0.192 and -0.122 e. $\AA^{-3}$

\section{Tetrahydrothiophene 8t}

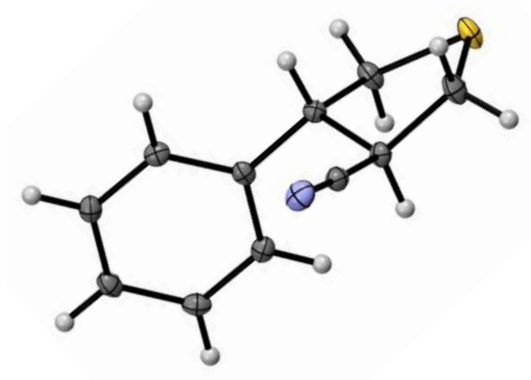

CCDC 1920381 contains the supplementary crystallographic data for 8t.

\section{Identification code}

Empirical formula

Formula weight

Temperature

Wavelength

Crystal system vv501

$\mathrm{C}_{11} \mathrm{H}_{11} \mathrm{NS}$

189.27

$100(2) \mathrm{K}$

$0.71073 \AA$

Monoclinic 
Space group

Unit cell dimensions

Volume

Z

Density (calculated)

$\mu$

Absorption correction

Crystal size

Transmission factor range

Reflections collected

$\mathrm{R}_{\text {int }}$

Mean $\sigma(I) / I$

$\theta$ range

Reflections observed

$x, y$ (weighting scheme)

Hydrogen refinement

Reflections in refinement

Parameters

Restraints

$R\left(F_{\text {obs }}\right)$

$R_{w}\left(F^{2}\right)$

$S$

shift/error $\max$

Max. electron density

Min. electron density
'P 121/c 1'

$\begin{array}{ll}a=5.4787(2) \AA & \alpha=90^{\circ} \\ b=9.4426(4) \AA & \beta=96.9810(10)^{\circ} \\ c=18.2072(6) \AA & \gamma=90^{\circ}\end{array}$

934.93(6) $\AA^{3}$

4

$1.345 \mathrm{Mg} / \mathrm{m}^{3}$

$0.293 \mathrm{~mm}^{-1}$

Multi-Scan

$0.080 \times 0.060 \times 0.030 \mathrm{~mm}^{3}$

0.9135-0.9705

10894

0.0345

0.0249

3.747-26.372

1704

0.0305 and 0.4493

Constr.

1904

118

0

0.0285

0.0736

1.079

0.001

0.262 e. $\AA^{-3}$

-0.259 e. $\AA^{-3}$ 


\section{Tetrahydrothiophene (-)-8z}

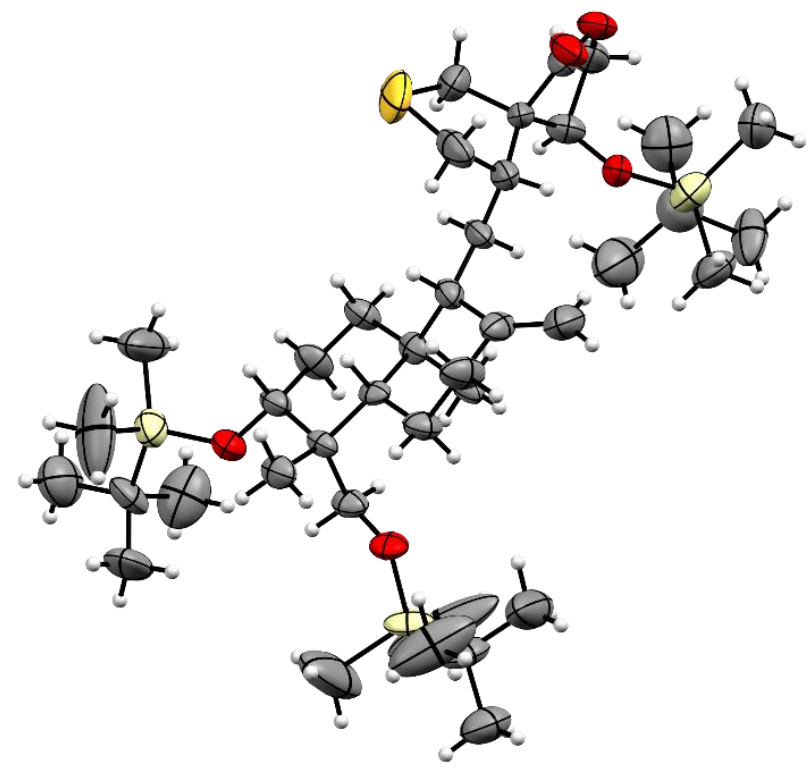

CCDC 1921469 contains the supplementary crystallographic data for (-)-8z.

Identification code

Empirical formula

Formula weight

Temperature

Wavelength

Crystal system

Space group

Unit cell dimensions

Volume

Z

Density (calculated)

Absorption coefficient

$\mathrm{F}(000)$

Crystal size

Theta range for data collection

Index ranges

Reflections collected

Independent reflections

Completeness to theta $=23.000^{\circ}$

Absorption correction mar19-5

$\mathrm{C}_{40} \mathrm{H}_{76} \mathrm{O}_{5} \mathrm{SSi}_{3}$

753.33

183(2) K

$0.71073 \AA$

Monoclinic

$\mathrm{P}_{21}$ (no. 4)

$\mathrm{a}=9.5103(6) \AA \quad \alpha=90^{\circ}$

$b=34.984(2) \AA \quad \beta=101.5185(17)^{\circ}$

$c=21.4829(14) \AA \quad Y=90^{\circ}$

7003.5(8) $\AA^{3}$

6

\section{$1.072 \mathrm{Mg} / \mathrm{m}^{3}$}

$0.183 \mathrm{~mm}^{-1}$

2484

$0.190 \times 0.180 \times 0.080 \mathrm{~mm}^{3}$

2.258 to $23.000^{\circ}$

$10<=\mathrm{h}<=9,-38<=\mathrm{k}<=38,-23<=\mathrm{k}<=23$

88070

$19490[R($ int $)=0.0473]$

$99.8 \%$

Semi-empirical from equivalents 
Max. and min. transmission

Refinement method

Data / restraints / parameters

Goodness-of-fit on $\mathrm{F}^{2}$

Final $R$ indices [l>2sigma(I)]

$\mathrm{R}$ indices (all data)

Absolute structure parameter

Extinction coefficient

Largest diff. peak and hole
0.958 and 0.882

Full-matrix least-squares on F2

$19490 / 15 / 1314$

1.036

$\mathrm{R} 1=0.0926, \mathrm{wR} 2=0.2484$

$\mathrm{R} 1=0.1204, \mathrm{wR} 2=0.2724$

$-0.01(2)$

$\mathrm{n} / \mathrm{a}$

1.347 and -0.867 e. $\AA^{-3}$

Tetrahydro-4H-thiopyran-4-one 11

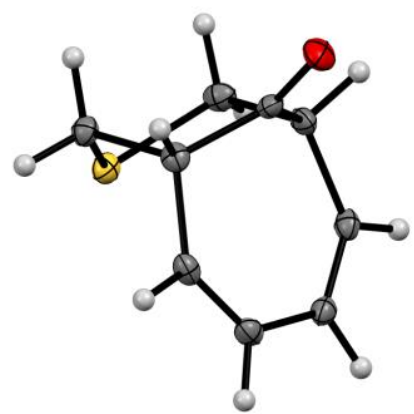

CCDC 1920380 contains the supplementary crystallographic data for 11 .

\begin{tabular}{lll}
\hline Identification code & vv500 & \\
Empirical formula & $\mathrm{C}_{9} \mathrm{H}_{10} \mathrm{OS}$ & \\
Formula weight & 166.23 & \\
Temperature & $100(2) \mathrm{K}$ & \\
Wavelength & $0.71073 \AA$ & \\
Crystal system & Monoclinic & \\
Space group & 'P $121 / \mathrm{c} 1^{\prime}$ & \\
Unit cell dimensions & $\mathrm{a}=9.8449(5) \AA$ & $\alpha=90^{\circ}$ \\
& $\mathrm{b}=6.6282(3) \AA$ & $\beta=91.542(2)^{\circ}$ \\
& $\mathrm{C}=12.4312(7) \AA$ & $\mathrm{V}=90^{\circ}$ \\
Volume & $810.89(7) \AA^{3}$ & \\
Z & 4 & \\
Density (calculated) & $1.258 \mathrm{Mg}^{3} \mathrm{~m}^{3}$ & \\
$\mu$ & $0.333 \mathrm{~mm}^{-1}$ & \\
Absorption correction & Multi-Scan & \\
Crystal size & $0.090 \times 0.060 \times 0.030 \mathrm{~mm}^{3}$ \\
Transmission factor range & $0.9014-0.9705$ & \\
& &
\end{tabular}


Reflections collected

$\mathrm{R}_{\text {int }}$

Mean $\sigma(I) / I$

$\theta$ range

Reflections observed

$x, y$ (weighting scheme)

Hydrogen refinement

Reflections in refinement

Parameters

Restraints

$R\left(F_{\text {obs }}\right)$

$R_{w}\left(F^{2}\right)$

$S$

shift/error max

Max. electron density

Min. electron density

\section{4}

0.0298

0.0274

3.279-26.367

1446

0.0215 and 0.5204

Constr.

1646

100

0

0.0283

0.0703

1.080

0.001

0.315 e. $\AA^{-3}$

-0.236 e. $\AA^{-3}$

\section{Dihydrothiophene $12 e$}

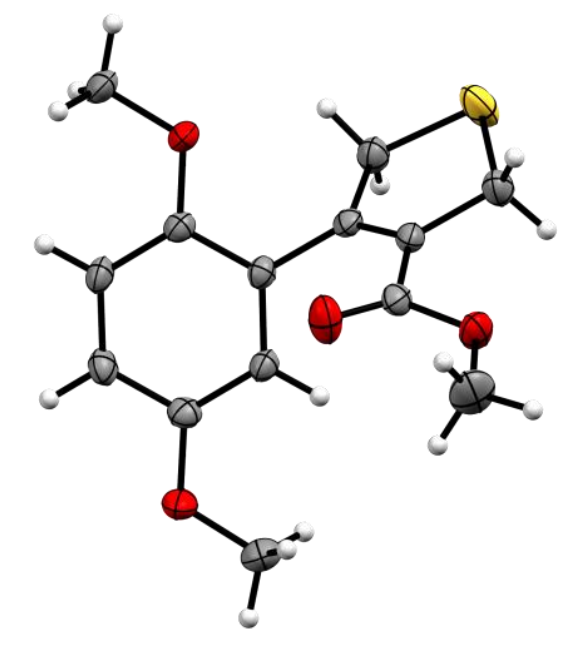

CCDC 1921470 contains the supplementary crystallographic data for $\mathbf{1 2 e .}$

\begin{abstract}
Identification code
Empirical formula

Formula weight

Temperature

Wavelength

Crystal system
\end{abstract}

Space group
mar19-2
$\mathrm{C}_{14} \mathrm{H}_{16} \mathrm{O}_{4} \mathrm{~S}$
280.33
223(2) K
$0.71073 \AA$

Monoclinic

P21/n (no. 14) 
Unit cell dimensions

Volume

Z

Density (calculated)

Absorption coefficient

$F(000)$

Crystal size

Theta range for data collection

Index ranges

Reflections collected

Independent reflections

Completeness to theta $=25.000^{\circ}$

Absorption correction

Max. and min. transmission

Refinement method

Data / restraints / parameters

Goodness-of-fit on $\mathrm{F}^{2}$

Final $\mathrm{R}$ indices [l>2sigma(I)]

$\mathrm{R}$ indices (all data)

Extinction coefficient

Largest diff. peak and hole

$$
\begin{array}{ll}
a=7.0480(3) \AA & \alpha=90^{\circ} \\
b=10.4797(5) \AA & \beta=97.094(2)^{\circ} \\
c=18.4167(10) \AA & V=90^{\circ}
\end{array}
$$$$
1349.86(11) \AA^{3}
$$

4

\section{$1.379 \mathrm{Mg} / \mathrm{m}^{3}$}

$0.247 \mathrm{~mm}^{-1}$

592

$0.350 \times 0.210 \times 0.180 \mathrm{~mm} 3$

2.229 to $25.000^{\circ}$

$-8<=\mathrm{h}<=8,-12<=\mathrm{k}<=12,-21<=\mathrm{l}<=21$

32811

$2380[R$ (int) $=0.0257]$

$99.9 \%$

Semi-empirical from equivalents

0.958 and 0.935

Full-matrix least-squares on $\mathrm{F}^{2}$

$2380 / 0 / 173$

1.072

$\mathrm{R} 1=0.0322, \mathrm{wR} 2=0.0835$

$\mathrm{R} 1=0.0346, \mathrm{wR} 2=0.0852$

$0.019(2)$

0.233 and -0.279 e. $\AA^{-3}$

\section{Diels-Alder adduct 20}

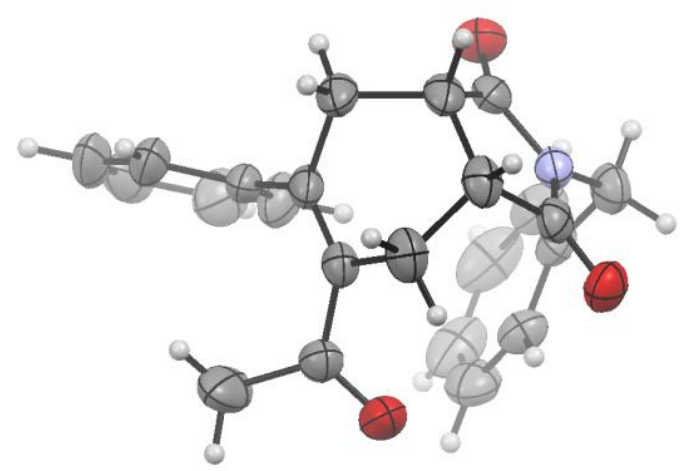

CCDC 1921471 contains the supplementary crystallographic data for 20.

Identification code

Empirical formula mar19-1

$\mathrm{C}_{23} \mathrm{H}_{21} \mathrm{NO}_{3}$ 
Formula weight

Temperature

Wavelength

Crystal system

Space group

Unit cell dimensions

Volume

Z

Density (calculated)

Absorption coefficient

$F(000)$

Crystal size

Theta range for data collection

Index ranges

Reflections collected

Independent reflections

Completeness to theta $=24.998^{\circ}$

Absorption correction

Max. and min. transmission

Refinement method

Data / restraints / parameters

Goodness-of-fit on $\mathrm{F}^{2}$

Final $R$ indices [l>2sigma(I)]

$\mathrm{R}$ indices (all data)

Absolute structure parameter

Extinction coefficient

Largest diff. peak and hole
359.41

223(2) K

$0.71073 \AA$

Orthorhombic

P212121 (no. 19)

$a=8.6617(4) \AA \quad \alpha=90^{\circ}$

$b=9.6496(4) \AA \quad \beta=90^{\circ}$

$\mathrm{C}=22.5991(9) \AA \quad \mathrm{Y}=90^{\circ}$

$1888.88(14) \AA^{3}$

4

$1.264 \mathrm{Mg} / \mathrm{m}^{3}$

$0.084 \mathrm{~mm}^{-1}$

760

$0.210 \times 0.180 \times 0.120 \mathrm{~mm}^{3}$

2.518 to $24.998^{\circ}$

$-10<=\mathrm{h}<=10,-11<=\mathrm{k}<=11,-26<=\mathrm{k}<=26$

22315

$3331[R($ int $)=0.0276]$

$99.9 \%$

Semi-empirical from equivalents

0.958 and 0.924

Full-matrix least-squares on $\mathrm{F}^{2}$

$3331 / 0 / 246$

1.034

$\mathrm{R} 1=0.0308, \mathrm{wR} 2=0.0790$

$\mathrm{R} 1=0.0334, \mathrm{wR} 2=0.0809$

$-0.4(3)$

$0.038(3)$

0.127 and -0.118 e. $\AA^{-3}$ 